NUREG/CR-4829

UCID-20733

Vol. 2

RT

\title{
Shipping Container Response to Severe Highway and Railway Accident Conditions
}

NUREG/CR--4829-VOI. 2

Appendices

Manuscript Completed: April 1986

Date Published: February 1987

Prepared by

L. E. Fischer, C. K. Chou, M. A. Gerhard, C. Y. Kimura,

R. W. Martin, R. W. Mensing, M. E. Mount, M. C. Witte

Lawrence Livermore National Laboratory

7000 East Avenue

Livermore, CA 94550

\section{Prepared for}

Division of Reactor System Safety

Office of Nuclear Regulatory Research

U.S. Nuclear Regulatory Commission

Washington, DC 20555

NRC FIN A0397
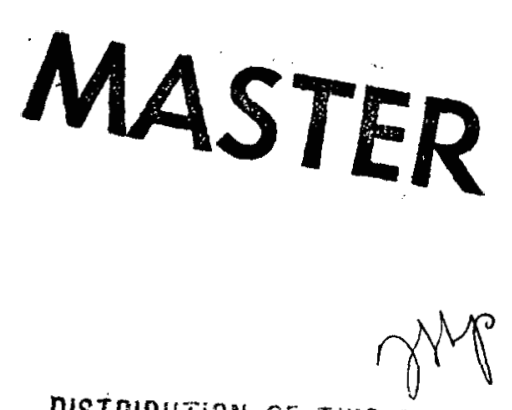


\section{DISCLAIMER}

This report was prepared as an account of work sponsored by an agency of the United States Government. Neither the United States Government nor any agency Thereof, nor any of their employees, makes any warranty, express or implied, or assumes any legal liability or responsibility for the accuracy, completeness, or usefulness of any information, apparatus, product, or process disclosed, or represents that its use would not infringe privately owned rights. Reference herein to any specific commercial product, process, or service by trade name, trademark, manufacturer, or otherwise does not necessarily constitute or imply its endorsement, recommendation, or favoring by the United States Government or any agency thereof. The views and opinions of authors expressed herein do not necessarily state or reflect those of the United States Government or any agency thereof. 


\section{DISCLAIMER}

Portions of this document may be illegible in electronic image products. Images are produced from the best available original document. 


\section{NOTICE}

This report was prepared as an account of work sponsored by an agency of the United States Government. Neither the United States Government nor any agency thereof, or any of their employees. makes any warranty, expressed or implied, or assumes any legal liability of responsibility for any third party's use, or the results of such use. of any information, apparatus. product or process disclosed in this report, or represents that its use by such third party would not infringe privately owned rights.

\section{NOTICE}

\section{Availability of Reference Materials Cited in NRC Publications}

Most documents cited in NRC publications will be available from one of the following sources:

1. The NRC Public Document Room, 1717 H Street, N.W Washington, DC 20555

2. The Superintendent of Documents, U.S. Government Printing Office, Post Office Box 37082. Washington, DC 20013.7082

3. The National Technical Information Service, Springfield, VA 22161

Although the listing that follows represents the majority of documents cited in NRC publications, it is not intended to be exhaustive.

Referenced documents available for inspection and copying for a fee from the NRC Public Document Room include NRC correspondence and internal NRC memoranda; NRC Office of Inspection and Enforcement bulletins, circulars; information notices, inspection and investigation notices: Licensee Event Reports; vendor reports and correspondence; Commission papers; and applicant and licensee documents and correspondence.

The following documents in the NUREG series are available for purchase from the GPO Sales Program: formal NRC staff and contractor reports, NRC-sponsored conference proceedings, and NRC booklets and brochures. Also available are Regulatory Guides, NRC regulations in the Code of Federal Regulations, and Nuclear Regulatory Commission /ssuances.

Documents available from the National Technical Information Service include NUREG series reports and technical reports prepared by other federal agencies and reports prepared by the Atomic Energy Commission, forerunner agency to the Nuclear Regulatory Commission.

Documents available from public and special technical libraries include all open literature items. such as books, journal and periodical articles, and transactions. Federal Register notices, federal and state legislation, and congressional reports can usually be obtained from these libraries.

Documents such as theses, dissertations, foreign reports and translations, and non-NRC conference proceedings are available for purchase from the organization sponsoring the publication cited.

Single copies of NRC draft reports are available free, to the extent of supply, upon written request to the Division of Technical Information and Document Control, U.S. Nuclear Regulatory Com. mission, Washington, DC 20555.

Copies of industry codes and standards used in a substantive manner in the NRC regulatory process are maintained at the NRC Library, 7920 Norfolk Avenue, Bethesda, Maryland, and are available there for reference use by the public. Codes and standards are usually copyrighted and may be purchased from the originating organization or, if they are American National Standards, from the American National Standards Institute, 1430 Broadway. New York, NY 10018. 


\begin{abstract}
This report describes a study performed by the Lawrence Livermore National Laboratory to evaluate the level of safety provided under severe accident conditions during the shipment of spent fuel from nuclear power reactors. The evaluation is performed using data from real accident histories and using representative truck and rail cask models that likely meet 10 CFR 71 regulations. The responses of the representative casks are calculated for structural and thermal loads generated by severe highway and railway accident conditions. The cask responses are compared with those responses calculated for the 10 CFR 71 hypothetical accident conditions. By comparing the responses it is determined that most highway and railway accident conditions fall within the 10 CFR 71 hypothetical accident conditions. For those accidents that have higher responses, the probabilities and potential radiation exposures of the accidents are compared with those identified by the assessments made in the "Final Environmental Statement on the Transportation of Radioactive Material by Air and other Modes," NUREG-0170. Based on this comparison, it is concluded that the radiological risks from spent fuel under severe highway and railway accident conditions as derived in this study are less than risks previously estimated in the NUREG-0170 document.
\end{abstract}




\section{TABLE OF CONTENTS}

Page

1. INTRODUCTION $\ldots \ldots \ldots \ldots \ldots \ldots \ldots \ldots \ldots \ldots \ldots \ldots \ldots \ldots \ldots \ldots \ldots \ldots \ldots \ldots \ldots \ldots \ldots$

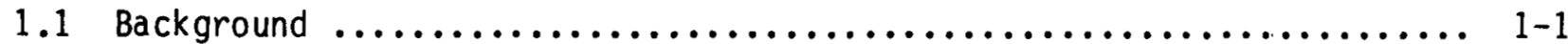

1.2 Regulations and Past Assessments $\ldots \ldots \ldots \ldots \ldots \ldots \ldots \ldots \ldots \ldots \ldots \ldots \ldots$

1.2.1 Title 10, Code of Federal Regulations, Part $71 \ldots \ldots \ldots \ldots \ldots \ldots$.

1.2.2 Transportation of Radioactive Material -

Environmental Statement (NUREG-0170).................. 1-7

1.3 Objective and Approach $\ldots \ldots \ldots \ldots \ldots \ldots \ldots \ldots \ldots \ldots \ldots \ldots \ldots \ldots$

2. ACCIDENT RATES, ACCIDENT SCENARIOS, AND LOADING PARAMETER

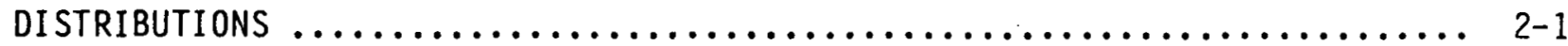

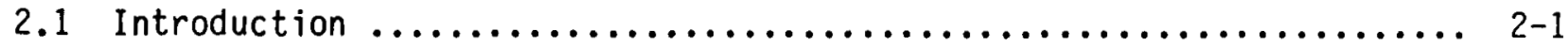

2.2 Highway Accident Rates ............................. 2-3

2.3 Railway Accident Rates $\ldots \ldots \ldots \ldots \ldots \ldots \ldots \ldots \ldots \ldots \ldots \ldots \ldots \ldots \ldots \ldots$

2.4 Accident Loading Data Requirements ...................... 2-4

2.5 Highway Accident Loading Parameters ........................ 2-10

2.5.1 Mechanical Loading Parameters ........................ 2-10

2.5.1.1 Accident Scenarios and 0bject Hardness ........... 2-10

2.5.1.1.1 Collision Accident Hardness Data ........ 2-11

2.5.1.1.2 Non-Collision Accident Hardness Data ..... 2-14

2.5.1.2 Impact Velocity ........................ 2-17

2.5.1.2.1 Cask Velocity ................... 2-17

2.5 .1 .2 .2 Impact Angle...................... 2-21

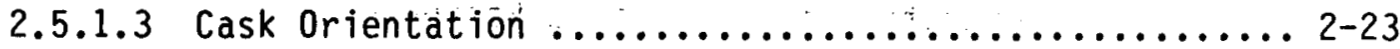

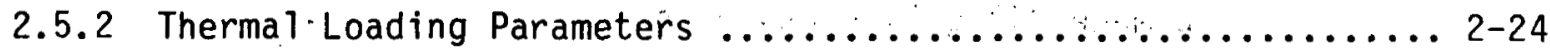

2.5.2.1 Accident Scenarios and Fire Frequency ..........2-24

2:5.2:2 Fire Duration ............................. 2-26

2.5.2.3 Flame Temperature ..................... 2-26

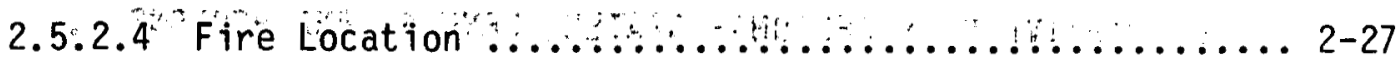

2.6 Rai 1way Accident Loading Parameters . . ....................... 2-27

2.6.1 Mechanical Loading Parämeters ...................... 2-27 
TABLE OF CONTENTS (cont inued)

Page

2.6.1.1 Accident Scenarios and Object Hardness ............ 2-28

2.6 .1 .2 Impact Velocity ........................ 2-30

2.6.1.2.1 Cask Velocity .................... 2-31

2.6.1.2.2 Impact Angle ................... 2-34

2.6.1.3 Cask Orientation ............................ 2-34

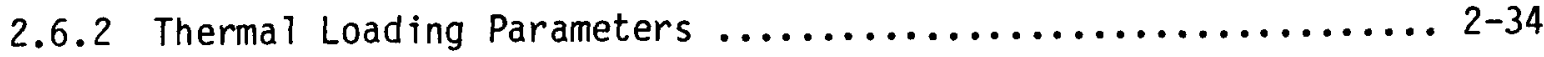

2.6.2.1 Accident Scenarios and Fire Frequency ........... 2-35

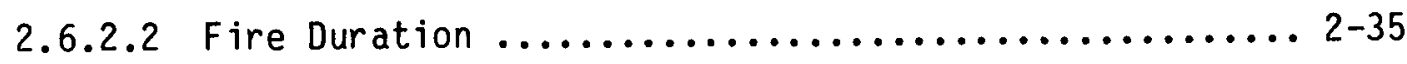

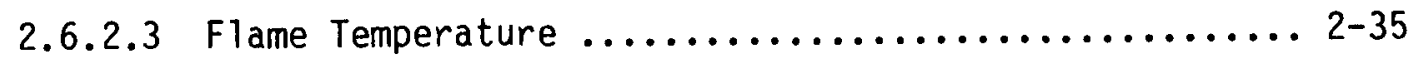

2.6 .2 .4 Fire Location ............................. 2-37

3. SELECTION OF REPRESENTATIVE SPENT FUEL CASKS FOR EVALUATION $\ldots \ldots \ldots \ldots \ldots . . . .3-1$

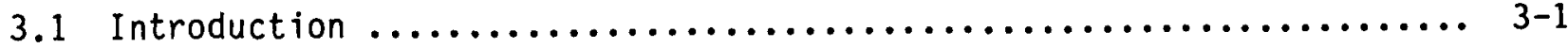

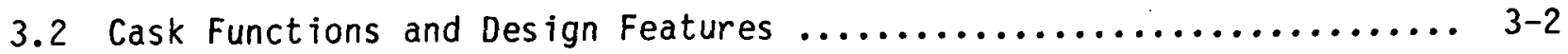

3.3 Cask Design Features Important to Safety .................... 3-5

3.3 .1 Containment ................................ 3-5

3.3 .2 Radiation Shielding $\ldots \ldots \ldots \ldots \ldots \ldots \ldots \ldots \ldots \ldots \ldots \ldots \ldots \ldots \ldots \ldots$

3.3 .3 Subcriticality Assurance $\ldots \ldots \ldots \ldots \ldots \ldots \ldots \ldots \ldots \ldots \ldots \ldots \ldots . \ldots . \ldots$

3.4 Selection of Cask Shielding Material ..................... 3-11

3.5 Definition of Representative Cask Designs .................... 3-14

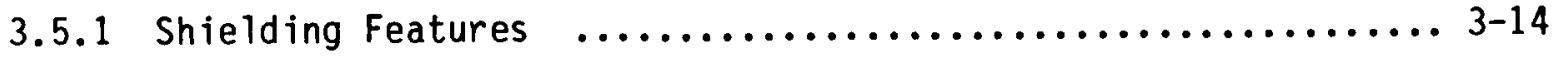

3.5 .2 Containment Features ................................ 3

3.5 .3 Subcritical ity Assurance Features $\ldots \ldots \ldots \ldots \ldots \ldots \ldots \ldots \ldots \ldots . \ldots . \ldots \ldots$

3.5 .4 Damage-Mitigat ing Features .......................... 3-17

3.5 .5 Representative Cask Design Description ................. 3-18

3.6 Margins of Safety.................................... 39

4. REPRESEnTATIVE CASK RESPONSE STATES, LEVELS, AND REgIONS ........... 4-1

4.1 Introduction $\ldots \ldots \ldots \ldots \ldots \ldots \ldots \ldots \ldots \ldots \ldots \ldots \ldots \ldots \ldots \ldots \ldots \ldots . \ldots \ldots$

4.2 Response States and Levels for Mechanical Loads ................ 4-2 
4.2.1 Structural Response Level, $s_{1} \ldots \ldots \ldots \ldots \ldots \ldots \ldots \ldots \ldots . . \ldots .4$

4.2 .2 Structural Response Lever, $S_{2} \ldots \ldots \ldots \ldots \ldots \ldots \ldots \ldots \ldots \ldots .44$

4.2 .3 Structural Response Level, $S_{3} \ldots \ldots \ldots \ldots \ldots \ldots \ldots \ldots \ldots . \ldots \ldots$ 4-6

4.2.4 Application of Response States and Levels .............. 4-6

4.3 Response States and Levels for Thermal Loads ................. 4-7

4.3.1 Thermal Response Level, $\mathrm{T}_{1} \ldots \ldots \ldots \ldots \ldots \ldots \ldots \ldots \ldots \ldots . \ldots .4$. 9

4.3.2 Therma1 Response Level, $\mathrm{T}_{2} \ldots \ldots \ldots \ldots \ldots \ldots \ldots \ldots \ldots \ldots \ldots .4 .11$

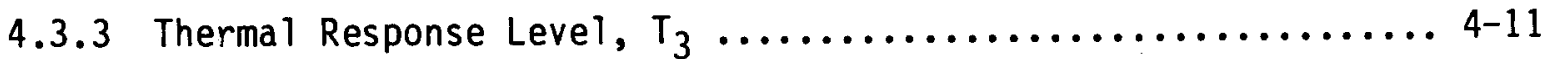

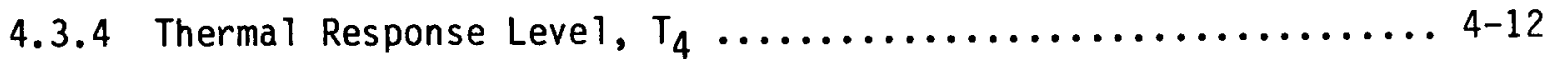

4.3.5 Application of Response States and Levels ............. 4-12

4.4 Cask Response Regions .............................. 4-14

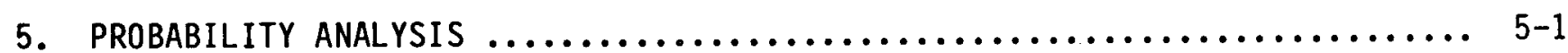

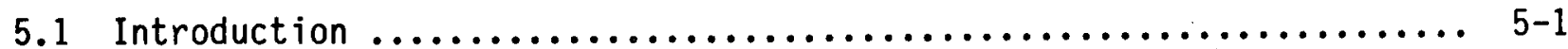

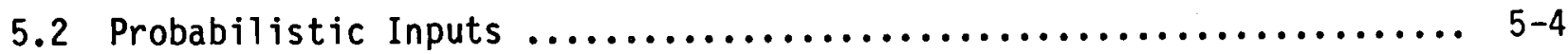

5.2.1 Mechanical Loading Parameter Distributions ............... 5-5

5.2.1.1 Object Hardness Distributions ................. 5-5

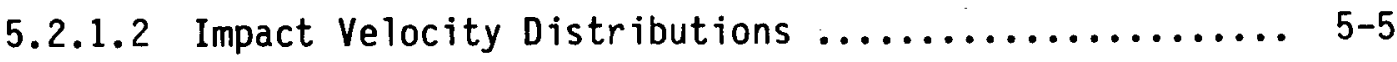

5.2.1.2.1 Cask Velocity ..................... 5-5

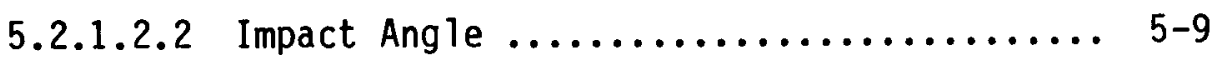

5.2.1.3 Cask Orientation Distributions ................ 5-11

5.2.2 Thermal Loading Parameter Distributions ................ 5-13

5.2.2.1 Fire Duration Distributions .................. 5-13

5.2.2.2 Flame Temperature Distributions ................ 5-15

5.2.2.3 Fire Location Distributions ................. 5-18

5.3 Probability Calculation ............................... 5-20

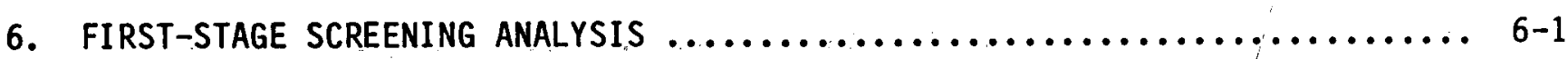

6.1 Introduction ........................................ $6-1$

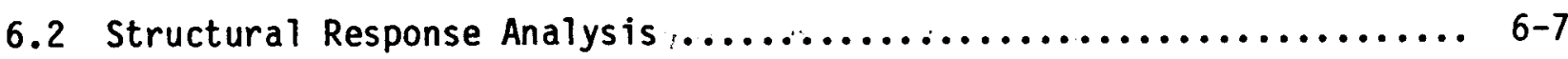




\section{TABLE OF CONTENTS (cont inued)}

\section{Page}

6.2.1 Cask Response Analys is for Highway Accidents ............6-15

6.2.1.1 Response to Minor Accidents................... 6-15

6.2 .1 .2 Response to Other Accidents .................. 6-16

6.2.1.2.1 Response for Impacts with Unyielding Surfaces .......................66-18

6.2.1.2.2 Response for Real Objects ...........6-18

6.2.2 Cask Response Analysis for. Railway Accidents ............6-21

6.2 .2 .1 Response to Minor Accidents.................. 6-23

6.2 .2 .2 Response to Other Accidents .................6-25

6.2.2.2.1 Response for Impacts with Unyielding Surfaces ...................... 6-25

6.2.2.2.2 Response for Real Objects ............6-27

6.2 .3 Discussion of Structural Analys is Results...............6-31

6.3 Thermat Response Anatysis .......................... 6-32

6.3.1 Cask Response Analys is for Highway Fire Accidents.........6 6-36

6.3.2 Cask Response Analysis for Railway Fire Accidents.......... 6-39

6.3 .3 Discussion of Thermal Analys is Results................6-43

6.4 Accident Screening Analysis.......................... 6-45

7. SECOND-STAGE SCREENING ANALYSIS $\ldots \ldots \ldots \ldots \ldots \ldots \ldots \ldots \ldots \ldots \ldots \ldots \ldots \ldots \ldots . \ldots . \ldots \ldots$

7.1 Introduction $\ldots \ldots \ldots \ldots \ldots \ldots \ldots \ldots \ldots \ldots \ldots \ldots \ldots \ldots \ldots \ldots \ldots \ldots$

7.2 Structural Response Analysis $\ldots \ldots \ldots \ldots \ldots \ldots \ldots \ldots \ldots \ldots \ldots \ldots \ldots . \ldots \ldots$

7.2.1 Cask Response Analys is for Highway Accidents ............ 7-4

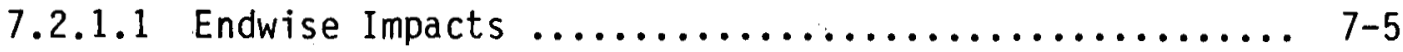

7.2 .1 .2 Sidewise Impacts ...................... 7-8

7.2.1.3 Impact Response Summary .................. 7-8

7.2.2 Cask Response Analys is for Railway Accidents ............ 7-12

7.2.2.1 Endwise Impacts ....................... 7-12

7.2.2.2. Sidewise Impacts ........................ 7-14

7.2.2.3 Impact Response Summary .................. 7-14 
7.2.3 Discussion of Structural Analys is Results $\ldots \ldots \ldots \ldots \ldots \ldots .7-14$

7.3 Thermal Response Analys is $\ldots \ldots \ldots \ldots \ldots \ldots \ldots \ldots \ldots \ldots \ldots \ldots \ldots \ldots \ldots \ldots \ldots \ldots \ldots \ldots .18$

7.3.1 Cask Response Analys is for Highway Fire Accidents ......... 7-19

7.3.2 Cask Response Analys is for Railway Fire Accidents ......... 7-21

7.3.3 Discussion of Thermal Analysis Results $\ldots \ldots \ldots \ldots \ldots \ldots \ldots .7-22$

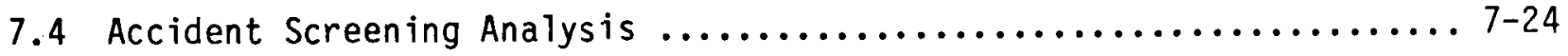

8. POTENTIAL RADIOLOGICAL SIGNIFICANCE OF TRANSPORTATION ACCIDENTS $\ldots \ldots \ldots . .8-1$

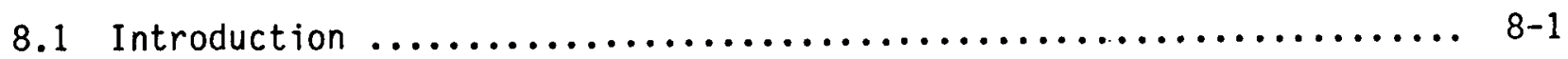

8.2 Description of Spent Fue $\ldots \ldots \ldots \ldots \ldots \ldots \ldots \ldots \ldots \ldots \ldots \ldots, 8,1$

8.3 Measures of Radiological Significance $\ldots \ldots \ldots \ldots \ldots \ldots \ldots \ldots \ldots \ldots . . .6$.

8.4 Estimates of Radiological Hazards $\ldots \ldots \ldots \ldots \ldots \ldots \ldots \ldots \ldots \ldots . . . \ldots .7$

8.4.1 Potential Radioactive Material Releases to the Environment ... 8-7

8.4.2 Potential Radiation Increases from Shielding Reduction....... 8-12

8.5 Radiological Effect Estimates for Response Regions ............. 8-18

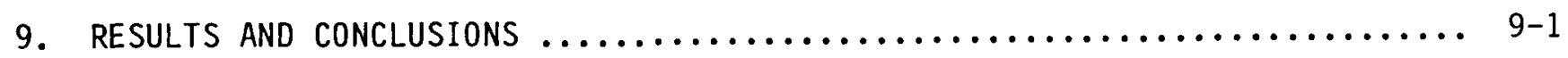

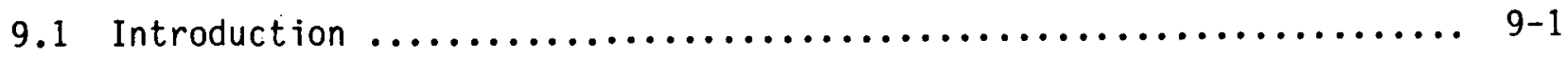

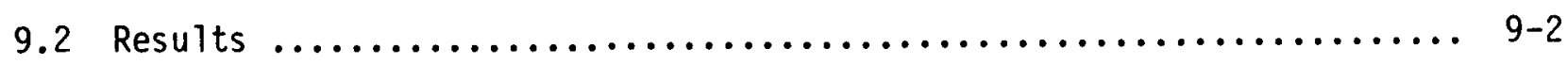

9.2 .1 First-Stage Screening .......................... $9-2$

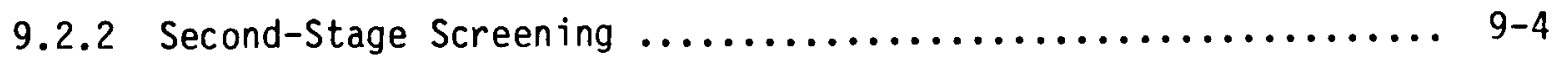

9.2.3 Comparison with Previous Risk Assessments: NUREG-0170 ...... 9-6

9.2.4 Est imated Responses for Sample Severe Accidents ........... 9-15

9.2.4.1 Caldecott Tunnel Fire . ..................... 9-15

9.2:4.2 I-80 Bridge Accident $\ldots \ldots \ldots \ldots \ldots \ldots \ldots \ldots \ldots \ldots .9 .16$

9.2.4.3 Livingston Train Fire ..................... 9-17

9.2.4.4 Derailment into the Alabama River .............. 9-18

9.3 Uncertainties ..................................... 9-19

9.3.1 Uncertainty in Cask Response ..................... 9-20

9.3.1.1 Selection of Representative Cask Designs .......... 9-20

9.3.1.2 Definition of Accident Loads .................. 9-21 
TABLE OF CONTENTS (cont inued)

Page

9.3.1.3 Computer Code Applications and Modeling .......... 9-21

9.3.2 Uncertainty in Estimating an Accident's Potential

Radiological Hazard ............................ 9-23

9.3.2.1 Radioactive Releases from Fuel Rods .............. 9-23

9.3.2.2 Radioactive Releases from Casks ............... 9-24

9.3.2.3 Reduction in Radiation Shielding ............... 9-24

9.3.2.4 Reduction in Subcriticality Control .............. 9-24

9.3.3 Uncertainty in Probability Models ..................... 9-25

9.3.3.1 Accident Statistics ...................... 9-25

9.3.3.2 Surveys of Structures and Features ............. 9-26

9.3 .3 .3 Past Analys is and Models .................. 9-26

9.3.3.4 Engineering Judgment .................... 9-27

9.3.4 Overal1 Statement of Uncertainty $\ldots \ldots \ldots \ldots \ldots \ldots \ldots \ldots \ldots \ldots . \ldots .27$

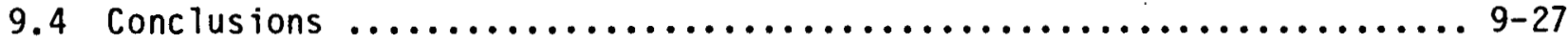

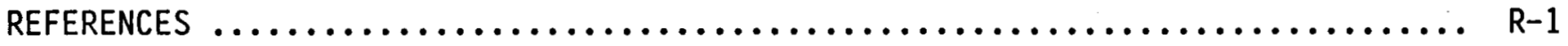

APPENDIX A: Severe Accident Data ............................ A-1

APPENDIX B: Truck Accident Data .................................. B-1

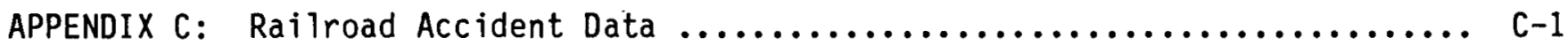

APPENDIX D: Highway Survey Data and Bridge Column Properties ............ D-1

APPENDIX E: Structural Anatys is .................................

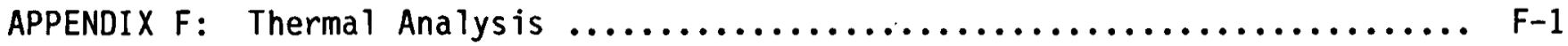

APPENDIX G: Probability Estimation Techniques ...................... G-1

APPENDIX $\mathrm{H}$ : Benchmarking for Computer Codes used in Impact Analys is ........ H-1 


\section{LIST OF FIGURES}

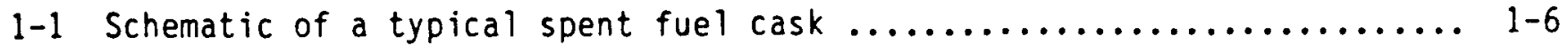

1-2 Two-stage screening process used in evaluating the regulations ......... 1-11

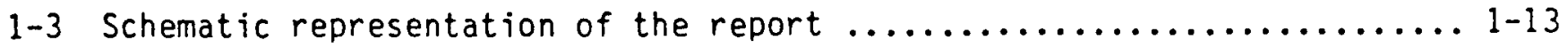

2-1 Three impact loading parameters considered in the response

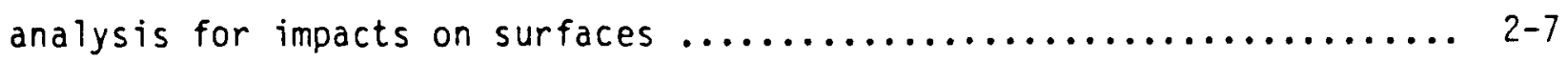

2-2 Three impact loading parameters considered in the response analysis for impacts with objects such as train sills $\ldots . \ldots \ldots \ldots \ldots .2-8$

2-3 Truck collision accident scenarios and their percent probabilities ........................................ 2-12

2-4 Truck non-collision accident scenarios and their percent

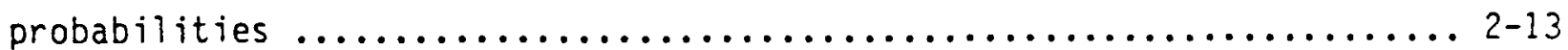

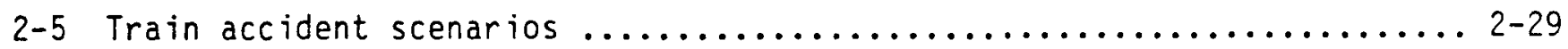

3-1 Spent fuel cask features important to safety $\ldots \ldots \ldots \ldots \ldots \ldots \ldots \ldots \ldots, 3-4$

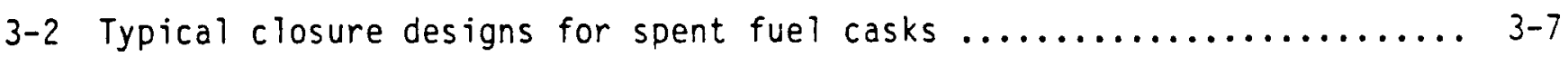

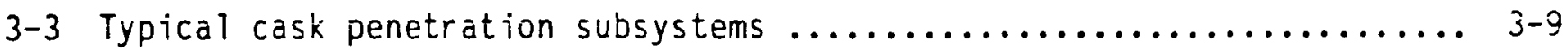

3-4 Preliminary truck cask designs with three types of gamma shielding, used for quasi-static loading response studies

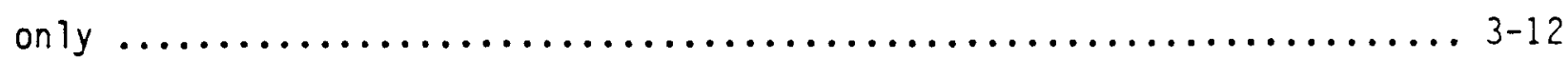

3-5 Preliminary rail cask designs with three types of gamma shielding, used for quasi-static loading response studies

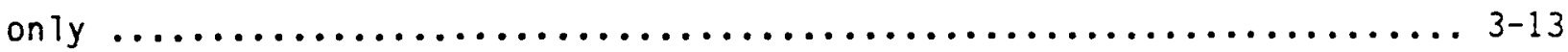

3-6 Representative truck cask design used for dynamic structura

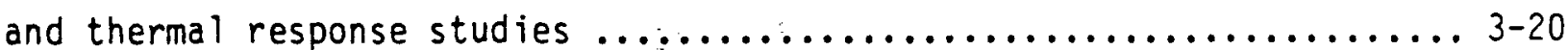

3-7 Representative rail cask design used for dynamic structural

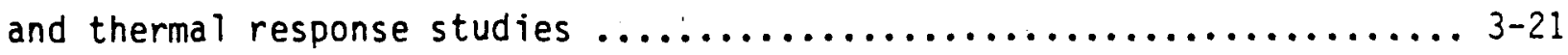

3-8 Force-deflection characteristics of the limiter design as a function of cask orientation at impact $\ldots \ldots \ldots \ldots \ldots \ldots \ldots \ldots . \ldots . . .22$ 


\section{LIST OF FIGURES (continued)}

Page

4-1 Schematic representation of cask response state for mechanical load ...................................... 4-5

4-2 Schematic representation of cask structural response for various surface hardness and impact velocities $\ldots . \ldots \ldots \ldots \ldots \ldots \ldots . \ldots . . . .4$

4-3 Schematic representation of cask response state for thermal

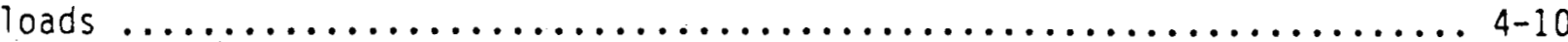

4-4 Schematic representation of cask response for various fire locations and fire durations .............................. $4-13$

4-5 Matrix of cask response regions for combined mechanical and

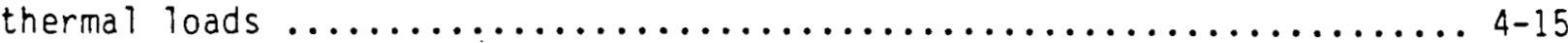

5-1 Effect of cask orientation on the strain-impact velocity relationship for a truck cask impacting an unyielding

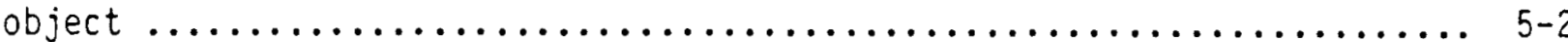

5-2 Effect of flame temperature and fire location on

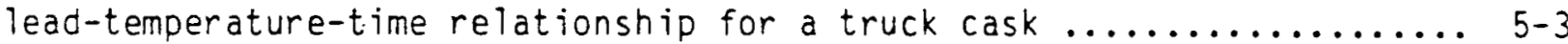

5-3 Distribution of vehicle velocities adjusted for braking ............ 5-7

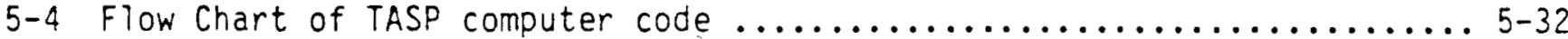

$6-1$ Identification of first-stage screening $\ldots \ldots \ldots \ldots \ldots \ldots \ldots \ldots \ldots \ldots \ldots .6 .2$

6-2 Methods of analysis used in cask response determinations ........... 6-5

6-3 Three impact loading parameters considered in the response analysis for impacts on surfaces $\ldots \ldots \ldots \ldots \ldots \ldots \ldots \ldots \ldots \ldots \ldots \ldots . \ldots .6$

6-4 Three impact loading parameters considered in the response analysis for impacts with objects such as train sills .............6-10

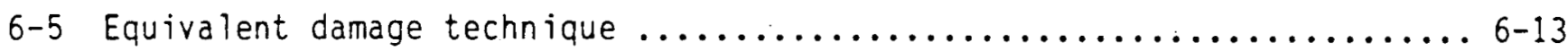

6-6 Strain versus impact velocity and cask orientation for the representative truck cask impacting an unyielding surface ...........6-19

6-7 Impact force for a rigid truck cask dropped endwise onto real surfaces 


\section{LIST OF FIGURES (cont inued)}

Page

6-8 Rail car coupler override of spent fuel cask car ................ 6-24

6-9 Strain versus impact velocity and cask orientation for the representative rail cask impacting an unyielding surface ...........6-28

6-10 Impact force versus impact velocity for a rigid rail cask dropped endwise onto real surfaces .......................... 6-29

6-11 Comparison of an engulfing hypothetical fire and a real fire ........6-34

6-12 Representative truck cask temperature response to a hypothetical $1475^{\circ} \mathrm{F}$ (equivalent to a real $1700^{\circ} \mathrm{F}$ ) fire

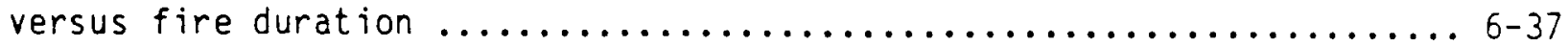

6-13 Heat flux versus fire duration for the representative truck cask exposed to the regulatory $1475^{\circ}$ fire $\ldots \ldots \ldots \ldots \ldots \ldots \ldots \ldots \ldots . \ldots 6$

6-14 Average heat flux factor versus temperature for the representative truck cask ..............................6-40

6-15 Heat load factor for real fire versus location of representative truck cask .............................. 6-41

6-16 Representative rail cask temperature response to a hypothetical $1475^{\circ} \mathrm{F}$ (equivalent to a real $1700^{\circ} \mathrm{F}$ ) fire versus fire duration $\ldots \ldots \ldots \ldots \ldots \ldots \ldots \ldots \ldots \ldots \ldots \ldots \ldots \ldots \ldots \ldots . \ldots \ldots$

6-17. Heat load factor for real fire versus location of representative rail cask ................................ 6-44

7-1 Second-stage screening analys is relationship with response regions $\ldots \ldots \ldots \ldots \ldots \ldots \ldots \ldots \ldots \ldots \ldots \ldots \ldots \ldots \ldots \ldots \ldots \ldots \ldots \ldots \ldots \ldots \ldots \ldots \ldots, 7-2$

7-2 Example showing strain response of the representative truck cask for $45 \mathrm{mph}$ endwise impact on an unyielding surface (2D model with impact limiters' ' without any truck cab crushing included $: \ldots \ldots \ldots \ldots . \ldots \ldots \ldots \ldots \ldots \ldots \ldots \ldots \ldots \ldots \ldots \ldots \ldots \ldots \ldots \ldots \ldots$

7-3 Response of the represeñative truck cask to endwise impacts on an unyielding surface " (2-0 model with impact

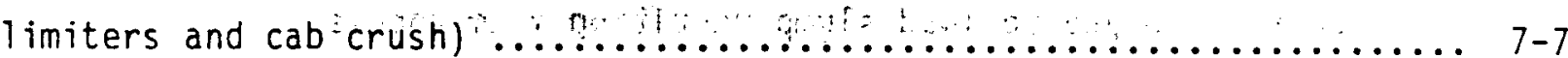




\section{LIST OF FIGURES (continued)}

Page

7-4 Example showing strain response of the representative truck cask for $60 \mathrm{mph}$ sidewise impact on soil (2-D model without limiters) with strain exceeding the $2 \%\left(s_{2}\right)$ limit $\ldots \ldots \ldots \ldots \ldots \ldots \ldots . . . .7$

7-5 Response of the representative truck cask to sidewise

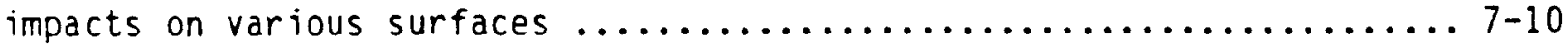

7-6 Response of the representative rail cask to endwise impacts on an unyielding surface (2-D model with impact limiters

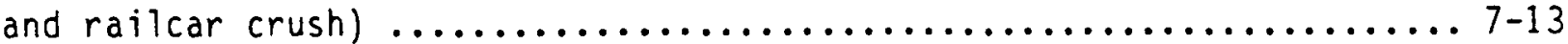

7-7 Response of the representative rail cask to sidewise impacts on various surfaces $\ldots \ldots \ldots \ldots \ldots \ldots \ldots \ldots \ldots \ldots \ldots \ldots \ldots \ldots \ldots \ldots \ldots$

7-8 Representative truck cask temperature response to a hypothetical $1475^{\circ} \mathrm{F}$ (equivalent to a real $1700^{\circ} \mathrm{F}$ ) fire

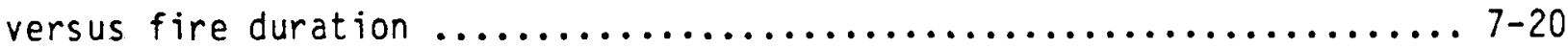

7-9 Representative rail cask temperature response to a hypothetical $1475^{\circ} \mathrm{F}$ (equivalent to a real $1700^{\circ} \mathrm{F}$ ) fire versus fire duration $\ldots \ldots \ldots \ldots \ldots \ldots \ldots \ldots \ldots \ldots \ldots \ldots \ldots \ldots \ldots \ldots \ldots . \ldots \ldots$

7-10 Fraction of truck accidents that could result in responses within each response region, assuming an accident occurs $\ldots \ldots \ldots \ldots \ldots .7-25$

7-11 Fraction of rail accidents that could result in responses within each response region, assuming an accident occurs $\ldots \ldots \ldots \ldots \ldots 7-26$

8-1 PWR fuel bundle $\ldots \ldots \ldots \ldots \ldots \ldots \ldots \ldots \ldots \ldots \ldots \ldots \ldots \ldots \ldots \ldots \ldots \ldots . \ldots \ldots$

8-2 Three mechanisms required to establish a radioactive material release path $\ldots \ldots \ldots \ldots \ldots \ldots \ldots \ldots \ldots \ldots \ldots \ldots \ldots \ldots \ldots . \ldots \ldots$

8-3 Percentage of fuel rods breached as a function of force for endwise impacts $\ldots \ldots \ldots \ldots \ldots \ldots \ldots \ldots \ldots \ldots \ldots \ldots \ldots \ldots \ldots \ldots \ldots$

8-4 Percentage of fuel rods breached per fuel assembly in each cask response region $\ldots \ldots \ldots \ldots \ldots \ldots \ldots \ldots \ldots \ldots \ldots \ldots \ldots \ldots \ldots \ldots . \ldots . \ldots . \ldots 1$

8-5 Lead voiding due to lead slump resulting from endwise impact of cask 


\section{LIST OF FIQURES (continued)}

8-6 Lead voiding due to high thermal loads and lead melting ............ 8-17

8-7 Radiological hazards estimated for response regions for a representative truck cask ................................. 8-19

8-8 Radiological hazards estimated for response regions for a

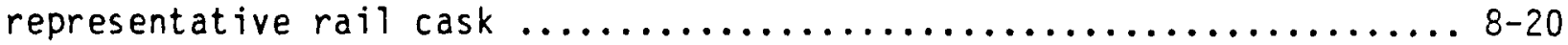

9-1 Two-stage screening process in the 20 response regions $. . . \ldots \ldots \ldots . . . .69$

9-2 Probability-hazard estimates in curies for the 20 truck

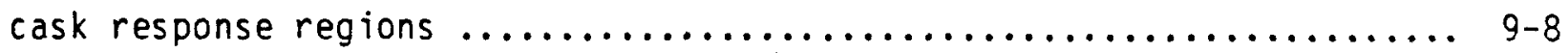

9-3 Probability-hazard estimates in curies for the 20 rail cask

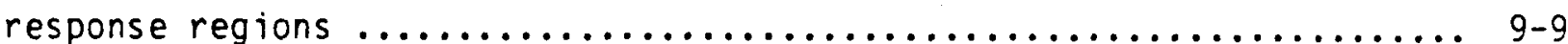




\section{LIST OF TABLES}

Page

1.1 Correlation of NUREG-0170 Accident Fractional Occurrence and Radiological Hazards as a Function of Accident Severity ......... 1-8

2.1 Accident Loads and Loading Parameters ...................... 2-5

2.2 Fractional Occurrence of Surface Types below Bridges on Interstate 80 from Davis, California to Nevada Border .............. 2-15

2.3 Distribution of Velocities for Trucks/Semitrailers Involved in Fatal and Injury Accidents in California, 1958-1967 ....................... 2-19

2.4 Distribution of Bridge Heights along Interstate 5 through Orange and Los Angeles Counties, California .....................2-20

2.5 Train Velocity Distribution for Rail-Highway Grade-Crossing Accident/Incidents Involving Motor Vehicles, 1975-1982 ........... 2-22

2.6 Frequency of Fire for Truck Accident Types .................... 2-25

2.7 Railroad Accident Velocity Distribution, Collisions, Main

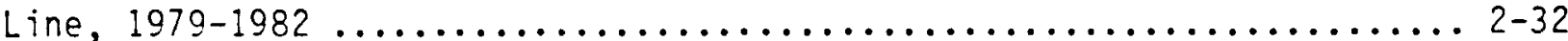

2.8 Railroad Accident Velocity Distribution, Derailments, Main

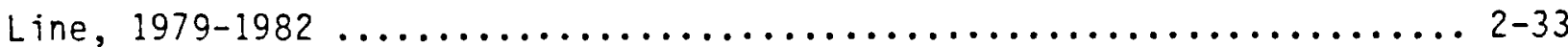

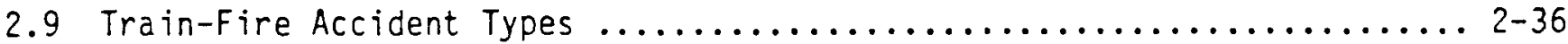

5.1 Cumulative Cask Velocity Distributions for Highway Analysis ......... 5-8

5.2 'Cumulative Cask Velocity Distributions for Railway Analys is .......... 5-10

5.3 Cumulative Impact Angle Distributions ....................... 5-12

5.4 Cumulative Cask Orientation Angle Distributions .................. 5-14

5.5 Cumulative Fire Duration Distributions for Truck Cask Analys is ...... 5-16

5.6 Cumulative Fire Duration Distributions for Rai T Cask Analys is ........ 5-17

5.7 Cumulative Flame Temperature Distribution .................. 5-19

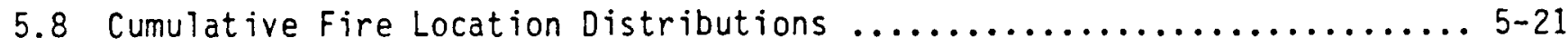

5.9 Probability Inputs for Highway Analys is .................... 5-25

5.10 Heat Flux Factors for Flame Temperatures (Engulfing Fire) .......... 5-28

5.11 Probability Inputs for Railway Analysis ..................... 5-31 


\section{LIST OF TABLES (continued)}

Page

6.1 Material Parameters Selected for Real Surfaces .................. 6-14

6.2 Evaluation of Quasi-Static Force for Minor Highway Accidents ......... 6-17

6.3 Impact Velocities Required to Reach the $0.2 \% \operatorname{Strain}\left(S_{1}\right)$

Level for Objects Impacted in Highway Accidents ................6-22

6.4 Evaluation Sumary of Minor Railway Accidents . ................6-26

6.5 Impact Velocities Required to Reach the $0.2 \%$ Strain $\left(S_{1}\right)$

Level for Objects Impacted in Railway Accidents ...............6-30

7.1 Impact Velocities Required to Attain $2 \%\left(S_{2}\right)$ and $30 \%\left(S_{3}\right)$

Strain Levels for Objects Impacted in Highway Accidents ........... 7-11

7.2 Impact Velocities Required to Attain $2 \%\left(S_{2}\right)$ and $30 \%\left(S_{3}\right)$

Strain Levels for Objects Impacted in Railway Accidents ........... 7-16

8.1 PWR Fuel Assembly Decay Heat and Radioactivity $\ldots \ldots \ldots \ldots \ldots \ldots \ldots \ldots . . .2$

8.210 CFR 71 Release Limits for Radioisotopes................... 8-6

8.3 Material Release Fractions from Breached Fuel Rods

Occurring over 1 week Following Rod Burst .....................8-13

8.4 Gamma Dose Summary for Lead Slump in a Rajl Cask for

Impacts on Closure Region ................................ 8

9.1 Comparative Measure of Risk/Accident for Spent Fue T

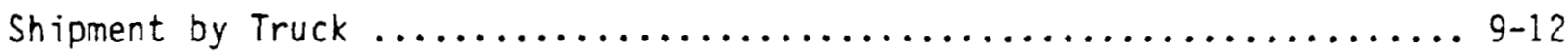

9.2 Comparison of Release Risk/Accident for Spent Fuel Shipment

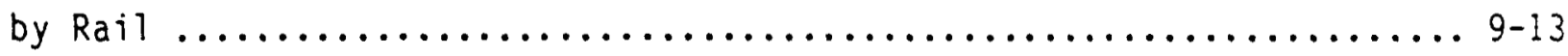




\section{PREFACE}

This report describes a study conducted to estimate the responses of spent fuel casks to severe highway and railway accident conditions and to assess the level of safety provided to the public during the shipment of spent fuel. The study was performed by the Lawrence Livermore National Laboratory for the U.S. Nuclear Regulatory Commission (NRC), Office of Nuclear Regulatory Research.

This report is divided into two volumes: Volume I, the main report, describes the study, the technical approach, the study results, and conclusions; and Volume II, the Appendixes, provide supporting accident data and engineering calculations. This report has been reviewed by the Denver Research Institute at the University of Denver under a separate contract to the NRC as the peer review. A companion summary report entitled "Transporting Spent Fuel-Protection Provided Against Severe Highway and Railway Accidents" (NUREG/BR-0111) has been prepared by the NRC for wide distribution to federal agencies, local governments, and interested citizens.

Commercial spent fuel shipments are regulated by both the Department of Transportation (DOT) and the NRC. The NRC evaluates and certifies the design, manufacture, operation, and maintenance of spent fuel casks, whereas the DOT regulates the vehicles and drivers which transport the spent fuel.

Current NRC regulations require spent fuel casks to meet certain performance standards. The performance standards include normal and hypothetical accident conditions which a cask must be capable of withstanding without exceeding established acceptance criteria that

(1) limit the release of radioactive material from the cask,

(2) limit the radiation levels external to the cask, and

(3) assure that the spent fuel remains subcritical.

This study evaluates the possible mechanical and thermal loads generated by actual and potential truck and railroad transportation accidents. The magnitudes of the loads from accidents are compared with the loads implied from the hypothetical accident conditions. The frequency of the accidents that can produce defined levels of mechanical and thermal loads are developed from the accident data base. Using this information, it is determined that $x i x$ 
for certain broad classes of accidents, spent fuel casks provide essentially complete protection against radiological hazards. For extremely severe accidents--those which could impose loads on the cask greater than those implied by the hypothetical accident conditions--the likelihood and magnitude of any radiological hazards are conservatively estimated. The radiological risk is then estimated and compared with risk estimates previously used by the NRC in judging the adequacy of its regulations.

The results of this study depend primarily on the quality of the cask response models, the radiation release models, and the probability models and distributions used in the analysis. Models for cask responses, radioactive releases, and distributions for the accident parameters are new developments based on current computer codes, limited test data on radioactive releases, and limited historical accident data. The results are derived using representative spent fuel casks which use design principles and materials that have been used in casks currently licensed by the NRC. The representative casks are assumed to have been designed, manufactured, operated, and maintained in accordance with national codes and standards (or equivalent) which have adequate margins of safety embedded in them. The results of this study are limited to spent fuel casks designed and fabricated under current technologies and operated under current regulations. New designs using alternative design principles and materials, or changes to regulations such as the imposition of a $75 \mathrm{mph}$ national speed limit, could affect the results and conclusions of this study.

This study does not consider the effects which human factors can have on the cask design, manufacture, operation, and maintenance. If further study is conducted, human factors should be considered because they can contribute to the overall risk in each phase of transporting spent fuel.

\section{E. Fischer}




\section{ACKNOWLEDGEMENTS}

The authors wish to acknowledge the technical contributions made to this report by R. C. Chun, L. L. George, T. E. Mckone, and M. W. Schwartz of Lawrence Livermore National Laboratory. The authors wish to thank G. E. Cummings of Lawrence Livermore National Laboratory for his report review and comments. The authors also wish to thank J. R. Cook, W. R. Lahs, and W. H. Lake of the U.S. Nuclear Regulatory Commission for their support and comments during the research and preparation of this report. Many thanks to N. J. Barnes and E. A. Sturmer for report preparation and D. Bowden for report editing.

In addition, the authors would particularly like to thank the following organizations for providing information and counsel which were used in preparing this report:

Anatech International Corporation

Association of American Railroads

Bureau of Motor Carrier Safety

Cal ifornia Department of Transportation

Central Electricity Generating Board, England

Denver Research Institute

Department of California Highway Patrol

Electric Power Research Institute

Engineering Computer Corporation

Federal Highway Administration

Federal Railroad Administration

Health and Safety Executive, England

Los Alamos National Laboratory

National Fire Protection Association

Oak Ridge National Laboratory

Ridihalgh, Eggers and Associates, Inc.

Sandia National Laboratories

Southern Pacific Transportation Company 


\section{APPENDIX A}

\section{List of Tables}

A.1 Caldecott Tunnel Fire Data Summary Sheet $. . . \ldots \ldots \ldots \ldots . \ldots \ldots \ldots$ A-4

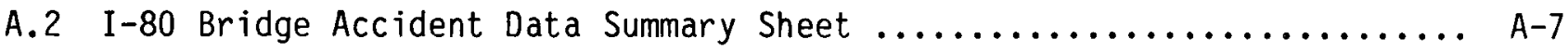

A.3 Livingston Train Fire Data Summary Sheet ..................... A-10

A.4 Alabama River Derailment Data Summary Sheet ................... A-14

A.5 Rail-Highway Grade-Crossing Accidents ........................ A-17

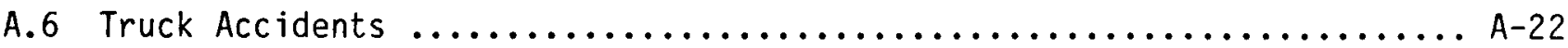

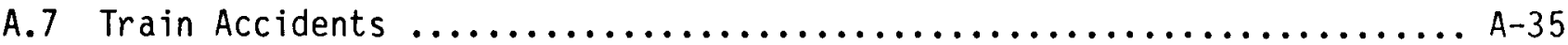


APPENDIX A

Severe Accident Data

\section{A.1 Introduction}

Under the first phase of the Nuclear Regulatory Commission Transportation Model Study Program, Ridihalgh, Eggers and Associates (REA) reviewed hundreds of severe highway and railway accident reports for the period from 1961 to 1981. A.1 Information on selected accidents was recorded onto a set of specially formatted data summary sheets. In this study, the severe accident data base was expanded to cover additional accidents in the $1980-1983$ period. The accident data compiled by REA was reviewed to sort out the information related to structural and thermal loading conditions. This appendix describes the process used to select severe accidents and presents sample data summary sheets for four severe accidents. Also summarized are all of the selected severe accidents with some of their more important loading parameters.

\section{A.2 Data Summary Sheets}

A literature search reported over 100,000 truck and train accidents in the period from 1961 to 1983. Approximately 335 accidents were selected for the period 1961 to 1981 , and 60 accidents were selected for the period 1981 to 1983. These accidents were judged to contain accident information that could be useful in assessing high physical loading conditions. All accidents had to involve either a truck or a train to be included in the selection process.

In generai, the information contained in the accident reports was more related to public safety issues: and the loss of life and property rather than to the physical loading conditions that occurred during: an accident. For example, a severe accident typically reported: could involve a truck and several cars resulting in a high loss of property and life, but could have occurred at moderate velocities (jess than $45 \mathrm{mph}$ ) and rioáding conditions that could have been relatively high to the cars $:(40,000=150,000$ pounds $), \therefore$ but relatively low to the tiruck. . On the other ihand, a runaway truck could hit a bridge abutment at high speed (greater than $80 \mathrm{mph}$ ) which could result in high 
loads (greater than 400,000 pounds), but never be included in a detailed national report because the loss of life and property would not be high, and the event would be so rare that it was not a public safety issue. All the compiled accident data were reviewed and the more important loading parameters that an accident can generate on a shipping container involved in such an accident are identified. Tables A.1 to A.4 present the data summary sheets for four typical severe accidents with high physical loading conditions.

The first data summary sheet, Table A.1, provides information on a truckfire accident in the Caldecott Tunnel near Oakland, California, in April 1982. The accident involved a gasoline truck-trailer, an automobile, and a bus. $A$ fire resulting from approximately 8,800 gallons of gasoline had a peak flame temperature of $1900^{\circ} \mathrm{F}$. Although the fire lasted 2 hours and 42 minutes according to the records, the peak flame temperature was estimated to have occurred for at least 20 minutes but not for the entire fire duration.

Table A.2 summarizes a truck-bridge accident, where in March 1981, a truck-tractor-trailer was struck by a pickup while on an overpass bridge on Interstate I-80 near San Francisco, California. The truck-tractor-trailer veered into the bridge railing, broke through the railing and fell 64 feet to the soil surface below.

Table A.3 provides information on a train fire accident, where on September 28, 1982, 43 railroad cars derailed near Livingston, Louisiana. Following the derailment, a fire started to burn various materials which included plastic pellets, vinyl chloride, and petroleum products. The fire which covered a wide area was allowed to burn for several days because of the toxic chemicals and explosions involved. A railroad car carrying motor fuel anti-knock compound (tetra-ethyl lead) exploded about 19 hours after the derailment. A second thermally induced explosion occurred on october 1, 82 hours after the derailment, involving a car carring vinyl chloride. The fire cooled down sufficiently on the fifth day to permit fire-fighting operations. Six cars carring chloride materials were purposely detonated on october 11 to dispose of the remaining unvented materials with in them. 
Finally, Table A.4 summarizes a train-bridge accident, where on January 19, 1979, a train derailed off a bridge into the Alabama River near Hunter, Alabama. One of the rail cars was carrying a pipe which struck the bridge and caused the derailment. Five rail cars fell into the river 75 feet below.

\section{A.3 Severe Accident Summary Tables}

Using the severe accident data summary sheets as input, tables were prepared summarizing each of the selected severe accidents to highlight the information related to loading magnitudes. Three different tables were prepared: Truck-Train Grade Crossing Accidents, Table A.5; Truck Accidents, Table A.6; and Rail Accidents, Table A.7.

Each accident is identified by its location (name of state and city) and is listed by its location in alphabetical order. For each accident the following information is provided: report source, date of accident, type of accident, number of vehicles involved, the velocity prior to the accident, the height of any fall involved, any object struck, and the duration of any fire involved. In some cases, the information was not stated on the data summary sheets and an NS is entered in the corresponding column.

\section{A.4 Reference}

A.1 P. Eggers, Severe Rail and Truck Accidents: Toward a Definition of Bounding Environment for Transportation Packages, U.S. Nuclear Regulatory Commission, Washington, DC, NUREG/CR-3499, October 1983 . 
Table A.1

Caldecott Tunnel Fire Data Summary Sheet

\subsection{ACCIDENT IDENTIFICATION}

1.01 Date of Accident: Apri1 7, 1982

1.02 Time of Accident: 0012

1.03 Rail, Highway or Both: Highway

1.04 Location: Caldecott Tunnel near Oakland, California

1.05 Railroad and/or Trucking Co. Invoived: Armour 0 il Company

1.06 Accident Report No.: NTSB/HAR-83/01, PB83-916201

1.07 : Source: NTSB

1.08 Title: HIGHWAY ACCIDENT REPORT - Multiple Vehicle Collisions and Fire Caldecott Tunnel near Oakland, California Apri1 7, 1982

1.09. Location of Document: REA

1.10 Location of Additional Information: NTSB

1.11 No. of Drawings/Photos: 16

\subsection{ACCIDENT EVENT DATA}

2.01 Initiating Event (derail, skid, overturn, explosion, collision, head to tail, head to head, tail to tail, head to side, fall): Head to tail collision

2.02 Cause: Intoxicated driver operating car, inattention of truck driver, excessive speed of bus

2.03 Number of Vehicles Involved: 1 truck and trailer, 1 car, 1 bus

2.04 Speed of Impact: Car stopped, truck $45 \mathrm{mph}$, bus $55 \mathrm{mph}$

2.05 Distance of Fall: Not applicable (N/A)

2.06 Weather Conditions: Clear

2.07 Ambient Temperature: $50^{\circ} \mathrm{F}$

2.08 Distance Traveled from Impact Point: Truck about $536 \mathrm{ft}$., bus about $2,175 \mathrm{ft}$

2.09 Description of Vehicles Involved: Cargo tank truck with full trailer and 5,400 gallon aluminum cargo tank, Gruman Flexible 53passenger bus, Honda Accord

2.10 Adjacent Structures or Natural Formations: Caldecott Tunne 1

2.11 Description of Cargo Involved in Accident: 8,800 gallons of gasoline, bus had no passengers

2.12 Elevation of Vehicles at Time of Accident: Highway through tunnel

2.13 Description of Surface Impacted: Truck to car, bus to car, bus to truck trailer, bus to highway support pier, car to tunnel wall

\subsection{SEQUENCE OF EVENTS}

3.01 Description of First Event: Honda car struck curb and stopped at left edge of roadway one-third of way through tunnel

3.02 Description of Second Event: Left front tire of tank trailer struck right rear corner of Honda 
3.03 Description of Third Event: Bus changed lanes and struck Honda and right front of the bus struck left side of the tank trailer

3.04 Description of Fourth Event: Trailer rolled over on right side and tank truck stops upright, gasoline spills

3.05 Description of Additional Events: Bus climbed left curb, traveled out of tunnel and impacted highway support pier. Gasoline spilled from trailer ignites.

3.06 Summary of Sequence of Events: I/A

\subsection{POST ACCIDENT EVENT DATA}

\subsection{POST ACCIDENT EVENT DATA}

4.1.01 Truck or Rail Car No. 1: Truck completely destroyed by fire, only remaining parts of cargo tank shell material included a 70 in by 96 in bottom sheet section from the rear compartment of the tank truck and a 40 in by $21 \mathrm{ft}$ section from the right side of the trailer tank. Left safety cable broken, main leaf springs deformed and separated from spring shackle.

4.1.02 Truck or Rail Car No. 2: Bus center front components displaced 17 $\mathrm{ft}$ rearward, front axle beam bent 6 inches rearward with axle and suspension attachment devices displaced and destroyed. Forward entrance door separated, forward front door post and hinge bar displaced 17 feet rearward.

4.1.03 Truck or Rail Car No. 3: Honda destroyed by fire.

4.1.04 Truck or Rail Car No. 4: N/A

4.1.05 Additional Trucks or Rail Cars Damaged: Tractor and utility semitrailer (beer truck), Ford pickup, Toyota pickup and Pontiac Phoenix sedan in tunnel incurred extensive fire damage but were not involved in collision.

4.1.06 Evidence of Crushing: N/A

4.1.07 Evidence of Impact: Left front tire of tank trailer struck right rear corner of Honda, Honda impacted tunnel wall, left front bumper of bus struck rear bumper of Honda, right front of bus struck left side of tank trailer, bus impacted highway support pier

4.1.08 Evidence of Falling: N/A

4.1.09 Evidence of Puncture: N/A

4.1.10 Evidence of Bending/Deformation of Support Members: Front axle beam of bus bent 6 inches

4.1.11 Evidence of Tearing Structural Members: N/A

4.1.12 Evidence of Projectiles Distance Traveled, Size/Weight of Projectile: N/A

4.1.13 Other Evidence of Severe Structural Damage: Tank truck and trailer tank destroyed, Honda destroyed, bus heavily damaged

\subsection{THERMAL/EXPLOSION DAMAGE DATA}

4.2.01 Type of Fire(s) and Fuel(s) Involved and Amounts: 8,800 gallons of gasoline 
4.2.02 Duration of Fire(s): 2 hours and 42 minutes

4.2.03 Evidence of Thermal Damage (e.g., melting, sagging or weakening): All low melting point and combustible material consumed by fire, only 2 sections of cargo tank shell material remained, examination of copper wires, aluminum casting, plastic parts, glass, glazed tile and concrete spalling provided a temperature determination in tunnel

4.2.04 Materials which Showed Evidence of Thermal Damage: Aluminum cargo tank

4.2.05 Evidence of Torch or Plume Fire: N/A

4.2.06 Evidence of Rocketing: N/A

4.2.07 Evidence of Explosions: Loud explosions were heard, lights went out, tiles fell from wall, final explosion shook building

4.2.08 No. of Vehicles Affected by Fires, Explosions: 1 cargo tank truck and tank trailer, 1 bus, 2 automobiles, 1 beer truck, 2 pickup trucks

4.2.09 Approximate Area Covered by Flames: 1,900 ft of tunnel

4.2.10 Evidence of Burial/Duration: N/A

\subsection{LEAK OR SPILL DATA}

4.3.01 Substance(s) Leaked or Spilled: Gasoline

4.3.02 Hazards/Damage Generated by Leakage/Spi11: Fire

4.3.03 Amount Leaked or Spilled: 8,800 gallons

4.3.04 Area Contaminated by Spi11: N/A

\subsection{MISCELLANEOUS OTHER DATA}

Fire produced temperature reaching $1900^{\circ} \mathrm{F}$ and remained that high for at least 20 minutes. Photos of damaged vehicles included in report.

\subsection{KEYWORD SUMMARY OF REPORT}


Table A.2

I-80 Bridge Accident Data Summary Sheet

\subsection{ACCIDENT IDENTIFICATION}

1.01 Date of Accident: March 1981

1.03 Rail, Highway or Both: Highway

1.04 Location: I-80, San Francisco Bay

1.05 Railroad and/or Trucking Co. Involved: Thomas M. Bonnell

Tractor/trailer

George A. Burris Pickup

1.07 Source: San Jose, California

1.08 Title: N/P Clipping

1.09 Location of Document: REA

1.10 Location of Additional Information: NTSB, BMCS, CHP

1.11 No. of Drawings/Photos: 1

\subsection{ACCIDENT EVENT DATA}

2.01 Initiating Event (derail, skid, overturn, explosion, collision, head to tail, head to head, tail to tail, head to side, fall):

Collision and loss of control
2.02 Cause: Not applicable (N/A)

2.04 Speed of Impact: $55 \mathrm{mph}$

2.05 Distance of Fall: 64 feet

2.09 Description of Vehicles Involved: Commercial Tractor/trailer, pickup truck

2.10 Adjacent Structures of Natural Formations: East Bay overpass

2.11 Description of Cargo Involved in Accident: N/A

2.12 Elevation of Vehicles at Time of Accident: On bridge roadway

2.13 Description of Surface Impacted: Tractor/trailer to pickup, tractor/trailer to concrete barrier, tractor/trailer to gravel and earth

\subsection{SEQUENCE OF EVENTS}

3.01 Description of First Event: Pickup truck veered in front of the tractor/trailer

3.02 Description of Second Event: Tractor/trailer. then struck the pickup and then itself. Tractor/trailer veered off the overpass, vaulted a concrete barrier and railing, and fell 64 feet.

\subsection{POST ACCIDENT EVENT DATA:}

\subsection{POST ACCIDENT EVENT DATA}

4.1.01 Truck or Rail Car No. 1: Tractor/trailer was demolished

4.1.02 Truck or Rail Car No. 2:A Pickup truck: was damaged

4.1.05 Additional Trucks or Rail Cars Damaged: 73 feet of rail and 12 feet of concrete barrier was torn out of bridge 
4.1.06 Eviderce of Crushing: N/A

4.1.07 Evidence of Impact: Tractor/trailer collided first with pickup truck then with bridge barrier and finally with earth

4.1.08 Evidence of Falling: 64 feet from bridge to earth

4.1.09 Evidence of Puncture: N/A

4.1.10 Evidence of Bending/Deformation of Support Members: N/A

4.1.11 Evidence of Tearing Structural Members: N/A

4.1.12 Evidence of Projectiles Distance Traveled, Size/Weight of Projectile: None

4.1.13 Other Evidence of Severe Structural Damage: N/A

\subsection{THERMAL/EXPLOSION DAMAGE DATA}

4.2.01 Type of Fire(s) and Fuel(s) Involved and Amounts: None

4.2.05 Evidence of Torch or Plume Fire: None

4.2.06 Evidence of Rocketing: None

4.2.07 Evidence of Explosions: None

\subsection{LEAK OR SPILL DATA}

4.3.01 Substance(s) leaked or spilled: N/A

\subsection{MISCELLANEOUS OTHER DATA}

\subsection{KEYWORD SUMMARY OF REPORT}

6.01 Vehicle class $(R=$ rail, $T=$ truck, $C=$ rail \& truck $): T$

6.02 Speed of Impact: $55 \mathrm{mph}$

6.03 Falling Distance: 64 feet

6.05 Impacting object ( $\mathrm{I} 1=$ locomotive, $\mathrm{I} 2=$ coupler, $\mathrm{I} 3=\mathrm{si11}, \mathrm{I} 4=$ axle, $I 5=$ bar stock, $I 6=$ plate stock, $I 7=I$-beam, $I 9=$ rail, $I 10=$ forging/casting, $I 11=$ tractor, $I 12=$ trailer, $I 13=$ no evidence of impacted object, $I 14=$ caboose, $I 15=$ other): I11 112

Object Impacted $(01=$ locomotive, $02=$ nox car, $03=$ tank car, 04 $=$ coal car, 05 = tractor, $06=$ trailer, $07=$ cargo, $08=$ cask, 09 = structural concrete, $010=$ building, $011=$ bridge, $012=$ automobile, 013 = no evidence of impacted object, 014 = caboose, 015 = other): 011015

6.08 Fire Duration: 0 minutes

6.09 Torch Duration: 0 minutes

6.10 Rocketing Distance: 0 feet

6.11 Weight of Rocketed Object: 0 pounds

6.12 Burial Event $(B 1=$ evidence of burial larger than 24 hours, $B 2=$ evidence of burial shorter than 24 hours, $B 3=$ no evidence of burial): B3

6.13 Ambient Temperature: $0^{\circ} \mathrm{F}$

6.16 Number of Fatalities: 0

6.17 Vehicle Type Involved in Accident ( $V 1=$ unit train, $V 2=$ passenger train, $V 3=$ mixed train cargo, $V 4=$ tractor trailer, $V 5=$ tandem trailer, $V 6=$ unit truck, $V 7=$ other $): V 4$ 
6.18 Cargo Type Involved in Accident $(Z 1=$ flammable, $Z 2=$ explosive, $\mathrm{Z3}=$ toxic, $\mathrm{Z4}=$ ordnance, $\mathrm{Z5}=$ radioactive, $\mathrm{Z6}=$ other $): \mathrm{Z6}$ 
Table A.3

Livingston Train Fire Data Sumiary Sheet

\subsection{ACCIDENT INFORMATION}

1.01 Date of Accident: September 28, 1982

1.02 Time of Accident: 0512

1.03 Rail, Highway or Both: Rail

1.04 Location: Livingston, Lousiana

1.05 Railroad and/or Trucking Co. Involved: Illinois Central Gulf Railroad

1.06 Accident Report No.: NTSB/RAR-83/05, PB83-916305

1.07 Source: NTSB

1.08 Title: RAILROAD ACCIDENT REPORT - Derailment of Illinois Central Gulf Railroad Freight Train Extra 9629 East (GS-2-28) and Release of Hazardous Materials at Livingston, Louisiana, September 28, 1982

1.09 Location of Document: REA

1.10 Location of Additional Information: NTSB

1.11 No. of Drawings/Photos: 11

\subsection{ACCIDENT EVENT DATA}

2.01 Initiating Event (derail, skid, overturn, explosion, collision, head to tail, head to head, tail to tail, head to side, fall): Derai1

2.02 Cause: Disengagement of air hose coupling, excessive buff force, placement of empty cars in train profile

2.03 Number of Vehicles Involved: 1 train

2.04 Speed of Impact: $40 \mathrm{mph}$

2.05 Distance of Fal1: Not applicable (N/A)

2.06 Weather Conditions: Clear

2.07 Ambient Temperature: $57^{\circ} \mathrm{F}$

2.08 Distance Traveled from Impact Point: N/A

2.09 Description of Vehicles Involved: Extra 9629 East consisting of 3 locomotive units, 84 loaded cars, 16 empty cars and a caboose, 29 cars were tank cars loaded with hazardous materials and 5 tank cars with flammable petroleum products

2.10 Adjacent Structures or Natural Formations: Smal1 community with buildings and pine groves surrounding tracks

2.11 Description of Cargo Involved in Accident: Plastic pellets, petroleum products, vinyl chloride, chemical products, styrene monomer, motor fuel anti-knock compound, toluene diisocyanate, phosphoric acid, hydrofluosilicic acid, sodium hydroxide, perchloroethylene, ethylene g fycol

2.12 Elevation of Vehicles at Time of Accident: Railroad bed 47 foot above sea level

2.13 Description of Surface Impacted: Gondola car to gondola car, tank car to railroad bed 


\subsection{SEQUENCE OF EVENTS}

3.01 Description of First Event: Train arrives Livingston and bottoms out at 2 crossings. Train went into emergency braking, automatic brake put into emergency position and throttle placed in ? position

3.02 Description of Second Event: 43 cars derail breaching 2 cars loaded with vinyl chloride

3.03 Description of Third Event: Leaking vinyl chloride gas ignites creating fireball exceeding $100 \mathrm{ft}$ south and $150 \mathrm{ft}$ north.

3.04 Description of Fourth Event: Explosion occurs and numerous fires break out

3.05 Description of Additional Events: Evacuation of area begun, hazardous materials unit notified and begin work. Next day tank car containing anti-knock compound explodes and rockets. September 30 fires intensify and vinyl chloride begins venting. October 1 vinyl chloride car explodes and rockets. October 4 styrene monomer re-ignites. October 5 styrene burns off and burning oil cars extinguished. October 10 and 11 vinyl chloride cars detonated. October 12 residents allowed to return. October 16 last derailed cars removed from accident site.

3.06 Summary of Sequence of Events: N/A

\subsection{POST ACCIDENT EVENT DATA}

\subsection{POST ACCIDENT EVENT DATA}

4.1.01 Truck or Rail Car No. 1: 19th and 20th cars detached from their trucks. 20th car had a vertical crease the full height

4.1.02 Truck or Rail Car No. 2: 3 tank cars loaded with petroleum products separated from their trucks and heavily damaged. 1 of these was breached.

4.1.03 Truck or Rail Car No. 3: Next 15 cars separated from their trucks and were damaged beyond economical repair

4.1.04 Truck or Rail Car No. 4: Next 18 cars were tank cars loaded with chemical products and were heavily damaged. 16 were punctured or breached.

4.1.05 Additional Trucks or Rail Cars Damaged: 5 cars had minor damage, 13 more cars separated from trucks, 15 tank cars had bottom out let extensions sheared off

4.1.06 Evidence of Crushing: N/A

4.1.07 Evidence of Impact: Vertical crease full height of gondola car, tank cars overturned, several cars destroyed by impact

4.1.08 Evidence of Falling: N/A $r$ :

4.1.09 Evidence of Puncture: 20 tank. cars punctured or breached, shell punctures in car containing perchioroethylene

4.1.10 Evidence of Bending/Deformations of Support Members: 36 cars destroyed by crushing impacts during derailment or by postaccident fires 
4.1.11 Evidence of Tearing Structural Members: 33 tank cars separated from trucks and many breached

4.1.12 Evidence of Projectiles Distance Traveled, Size/Weight of Projectile: Shell of tank car carrying anti-knock compound propelled about $80 \mathrm{ft}$ north and its tank head about $25 \mathrm{ft}$ south and most of its tub portion rocketed $425 \mathrm{ft}$ north. Large section of steel outer insulating jacket found about $80 \mathrm{ft}$ away. Other parts found $1,500 \mathrm{ft}$ south

4.1.13 Dther Evidence of Severe Structural Damage: 36 cars destroyed either by crushing impacts during the derailment or by postaccident fires, explosions, and demolition. Empty gondola car had vertical separation of bolster center plates.

\subsection{THERMAL/EXPLOSION DAMAGE DATA}

4.2.01 Type of Fire(s) and Fuel(s) Involved and Amounts: Vinyl chloride 163,043 gallons, styrene monomer 28,145 gallons, motor fuel antiknock compound (tetra-Ethyl lead) 5,666 gallons, toluene diisocyanate 2,259 gallons. Fires ailso fed by plastic pellets

4.2.02 Duration of Fire $(s): 8$ days

4.2.03 Evidence of Thermal Damage (e.g., melting, sagging or weakening): 2 thermaliy induced explosions

4.2.04 Materials which Showed Evidence of Thermal Damage: N/A

4.2.05 Evidence of Torch or Plume Fire: Vinyl chloride gas vented and burned from domes

4.2.06 Evidence of Rocketing: Thermaliy-induced explosions of 2 tank cars that had not been punctured caused them to rocket violently.

4.2.07 Evidence of Explosions: First explosion blew in brick front of dwelling $250 \mathrm{ft}$ north. 2 other thermally induced explosions.

4.2.08 No. of Vehicles Affected by Fires, Explosions: 13 train cars

4.2.09 Approximate Area Covered by Flames: 1,000 ft radius of derailment

4.2.10 Evidence of Burial/Duration: N/A

\subsection{LEAK OR SPILL DATA}

4.3.01 Substance(s) Leaked or Spilled: Phosphoric acid 148,552 gallons, hydrofluosilicic acid 19,780 gallons, sodium hydroxide 15,363 gallons, perchloroethylene 14,028 gallons, ethylene glycol 20,840 gallons, plastic pellets.

4.3.02 Hazards/Damage Generated by Leakage/Spil1: Acrid smoke and toxic gases as well as danger of fire and explosions

4.3.03 Amount Leaked or Spilled: More than 200,000 gallons of toxic chemical products

4.3.04 Area Contaminated by Spi11: Several acres containing more than 60,000 cubic yards of soil to be expected

\subsection{MISCELLANEOUS OTHER DATA}

Photos of accident and information on chemical compounds included in report. 9999 in fields 6.8 and 6.9 indicates time frame longer 
than 6 days. See 4.2.02. 3,000 people within 5-mile radius evacuated as long as 2 weeks

\subsection{KEYWORD SUMMARY OF REPORT}

6.01 Vehicle Class $(R=$ rail, $T=$ truck, $C=$ rail \& truck): $R$

6.02 Speed of Impact: $40 \mathrm{mph}$

6.03 Falling Distance: 0 feet

6.04 Crushing Events $(\mathrm{C} 1=$ locomotive, $\mathrm{C} 2=$ box car, $\mathrm{C} 3=\mathrm{coa}$ ) $\mathrm{car}, \mathrm{C} 4$ $=$ flat $c a r, C 5=\operatorname{tank} C a r, C 6=$ tractor, $C 7=$ trailer, $C 8=$ unit truck, $\mathrm{C9}=$ heavy cargo, $\mathrm{C} 10=$ tank truck, $\mathrm{C} 11=$ bridge, $\mathrm{Cl}=$ heavy support structure, $\mathrm{Cl3}=$ no evidence of crushing, $\mathrm{Cl} 4=$ caboose, $C 15=$ other): $C 5$

6.05 Impacting object (II = locomotive, $I 2=$ coupler, $I 3=$ sill, $I 4=$ axle, $I 5=$ bar stock, I6 = plate stock, I7 = I-beam, I9= rail, $I 10=$ forging/casting, $I 11=$ tractor, $I 12=$ trailer, $I 13=$ no evidence of impacted object, I14 = caboose, I15 = other): I2 I15

6.06 0bject Impacted $(01=$ locomotive, $02=$ box car, $03=$ tank car, 04

$=$ coal car, $05=$ tractor, $06=$ trailer, $07=$ cargo, $08=$ cask, 09

= structural concrete, $010=$ building, $011=$ bridge, $012=$ automobile, $0113=$ no evidence of impacted object, 014 = caboose, $015=$ other $): 0302$

6.07 Explosion Event (significant damage to: $E 1=$ train or truck vehicles, $E 2$ = surrounding structural members, $E 3=$ cratering of ground, $E 4=$ cargo, $E 5=$ none): $E 1$ E2 E4

6.08 Fire Duration (note: if 9,999 - see section 4.2.02): 9,999 minutes

6.09 Torch Duration (note: if 9,999 - see section 4.2.02): 9,999 minutes

6.10 Rocketing Distance: 425 feet

6.11 Weight of Rocketed object: 10,000 pounds

6.12 Burial Event ( $B 1=$ evidence of burial 1arger than 24 hours, $B 2=$ evidence of burial shorter than 24 hours, $B 3=$ no evidence of burial): $B 3$

6.13 Ambient Temperature: $57^{\circ} \mathrm{F}$

6.14 Vehicle Damage (thousands of dol lars): 1,500

6.15 Other Property Damage (thousands of dollars): 13,064

6.16 Number of Fatàlities: 0

6.17 Vehicle Type Involved in Accident ( $V 1=$ unit train, $V 2=$ passenger train, $V 3=$ mixed train cargo, $V 4=$ tractor trailer, $V 5=$ tandem trailer, $V 6=$ unit truck, $V 7=$ other): $V 3$

6.18 Cargo Type Involved in Accident $(Z 1=$ flamable, $Z 2=$ explosive, $Z 3=$ toxic, $Z 4=$ ordinance, $Z 5^{\prime}=$ radioactive, $Z 6=$ other $): ~ Z Z 1 Z 2$ Z3 Z6

6.19 CAS Registry Numbers for Cargo Involved An Accident None 
Table A.4

Alabama River Derailment Data Sumary Sheet

\subsection{ACCIDENT IDENTIFICATION}

1.01 Date of Accident: January 19, 1979

1.02 Time of Accident: 0740

1.03 Rail, Highway or Both: R

1.04 Location: Hunter, Alabama

1.05 Railroad and/or Trucking Co. Involved: Illinois Central Gulf Freight Train No. AM 118

1.06 Accident Report No.: ATL 78 F R018

1.07 Source: NTSB

1.08 Title: Accident File

1.09 Location of Document: REA

1.10 Location of Additional Information: NTSB

1.11 No. of Drawings/Photos: 2

\subsection{ACCIDENT EVENT DATA}

2.01 Initiating Event (derail, skid, overturn, explosion, collision, head to tail, head to head, tail to tail, head to side, fall): Collision with bridge

2.02 Cause: Improper lading

2.03 Number of Vehicles Involved: 72

2.04 Speed of Impact: $8 \mathrm{mph}$

2.05 Distance of Fall: 75 feet

2.06 Weather Conditions: Cloudy, dawn

2.07 Ambient Temperature: $45^{\circ} \mathrm{F}$

2.09 Description of Vehicles Involved: 3 locomotive units, 1 caboose, 2 blkd flat cars, 1 tank car, 46 loads, 19 empties

2.10 Adjacent Structures or Natural Formations: RR bridge over the Alabama River

2.11 Description of Cargo Involved in Accident: Two 54 in. 0.D.C.I. pipe cars, 1 tank car with fuel oil

2.12 Elevation of Vehicles at Time of Accident: RR bed on bridge deck

2.13 Description of Surface Impacted: Pipe to bridge, car to bridge, cars to river

\subsection{SEQUENCE OF EVENTS}

3.01 Description of First Event: Eight 54 in. pipes were strapped together in 2 groups of 4 each. The 2 groups laid in tandem

3.02 Description of Second Event: The pipes were then chained and blocked with wood sprags nailed to the car deck.

3.03 Description of Third Event: Sprags were not predrilled and later spl it loosening the load which was already unstable because of the "4-together" configuration. (Note: 3 pipes fastened in this fashion would have been stable). 
3.04 Description of Fourth Event: One of the loose pipe snagged the bridge superstructure bringing down one span

3.06 Summary of Sequence of Events: 5 loaded cars dropped into the Alabama River

\subsection{POST ACCIDENT EVENT DATA}

\subsection{POST ACCIDENT EVENT DATA}

4.1:01 Truck or Rail Car No. 1: 5 cars in river were damaged

4.1.02 Truck or Rail Car No. 2: Bridge was seriously damaged

4.1.06 Evidence of Crushing: None

4.1.07 Evidence of Impact: One of the 54 inch pipes impacted against a bridge truss

4.1.08 Evidence of Falling: 5 cars fell into the river as the bridge collapsed

4.1.09 Evidence of Puncture: Not applicable (N/A)

4.1.10 Evidence of Bending/Deformation of Support Members: N/A

4.1.11 Evidence of Tearing Structural Members: N/A

4.1.12 Evidence of Projectiles Distance Traveled, Size/Weight of Projectile: None

4.1.13 Other Evidence of Severe Structural Damage: See above

\subsection{THERMAL/EXPLOSION DAMAGE DATA}

4.2.01 Type of Fire(s) and Fuel(s) Involved and Amounts: None

4.2.05 Evidence of Torch or Plume Fire: None

4.2.06 Evidence of Rocketing: None

4.2.07 Evidence of Explosions: None

4.2.10 Evidence of Burial/Duration: Cars were in the river and mud

\subsection{LEAK OR SPILL DATA}

4.3.01 Substance(s) Leaked or Spilled: The tank car filled with fuel oil was reported not to be leaking

\subsection{KEYWORD SUMMARY OF REPORT}

6.01 Vehicle Class $(R=$ rail, $T=$ truck, $C=$ rail \& truck $): R$

6.02 Speed of Impact: $8 \mathrm{mph}$

6.03 Falling Distance: 75 feet

6.04 Crushing Events $(\mathrm{C} 1=$ locomotive, $\mathrm{C} 2=$ box, $\mathrm{C} 3=$ coal car, $\mathrm{C} 4=$ flat $\mathrm{car}, \mathrm{C5}=$ tank $\mathrm{car}, \mathrm{C6}=$ tractor, $\mathrm{C7}=$ trailer, $\mathrm{C8}=$ unit truck, $C 9=$ heavy cargo, $C 10=$ tank truck, $C 11=$ bridge, $C 12=$ heavy support structure, $\mathrm{C} 13=$ no evidence of crushing, $\mathrm{C} 14=$ caboose, $\mathrm{C} 15=$ other $): \quad$ C13

6.05 Impacting object ( $\mathrm{I} 1=$ locomotive, $\mathrm{I} 2=$ coupler, $\mathrm{I} 3=\mathrm{sil1,} \mathrm{I} 4=$ axle, $I 5=$ bar stock, I6 = plate stock, I7 = I-beam, I9= rail, $\mathrm{I} 10=$ forging/casting, $I 11=$ tractor, $I 12=$ trailer, $I 13=$ no evidence of impacted object, II4 = caboose, I15 = other): $I 10$ 
6.06 0bject Impacted $(01=$ locomotive, $02=$ box car, $03=$ tank car, 04 = coal car, $05=$ tractor, $06=$ trailer, $07=$ cargo, $08=$ cask, 09 = structural concrete, $010=$ building, $011=$ bridge, $012=$ automobile, $013=$ no evidence of impacted object, 014 = caboose, $015=$ other $): 011$

6.08 Fire Duration: 0 minutes

6.09 Torch Duration: 0 minutes

6.10 Rocketing Distance: 0 feet

6.11 Weight of Rocketed 0bject: 0 pounds

6.12 Burial Event ( $B 1=$ evidence of burial larger than 24 hours, $B 2=$ evidence of burial shorter than 24 hours, $B 3=$ no evidence of burial): Bl

6.13 Ambient Temperature: $45^{\circ} \mathrm{F}$

6.14 Vehicle Damage (thousands of dollars): 76

6.15 Other Property Damage (thousands of dollars): 2,000

6.16 Number of Fatalities: 0

6.17 Vehicle Type Involved in Accident (V1 = unit train, $V_{2}=$ passenger train, $V_{3}=$ mixed train cargo, $V_{4}=$ tractor trailer, $V_{5}=$ tandem trailer, $V 6=$ unit truck, $V 7=$ other $): V 3$

6.18 Cargo Type Involved in Accident $(Z 1=$ flamable, $Z 2=$ explosive, $Z 3=$ toxic, $Z 4=$ ordnance, $Z 5=$ radioactive, $Z 6=$ other $): Z 1 Z 6$ 


\section{Table A.5 Legend}

Rail-Highway Grade-Crossing Accidents

\section{Report Source}

FRA

Federal Railroad Administration

NATL, year, report \#. Department of Transportation, Federal Railroad Administration, Atlanta 0ffice

NCHI, year, report. \# .... Department of Transportation, Federal Railroad Administration, Chicago 0ffice $N / H A B$ National Transportation Safety Board, Highway Accident Brief

NOAK, year, report \# Department of Transportation, Federal Railroad Administration, Oakland 0ffice N/RHR. NS: National Transportation Safety Board, Railroad Highway Report

NTSB Not Stated

National Transportation Safety Board

$\stackrel{P}{\mathfrak{1}}$

\section{Accident Description}

HtoS : Col. Head to Side Collision

Vhcl. Vehicle 
Table A.5

Rail-Highway Grade-Crossing Accidents

\begin{tabular}{|c|c|c|c|c|c|c|c|c|}
\hline Location & $\begin{array}{l}\text { Report } \\
\text { Source }\end{array}$ & $\begin{array}{l}\text { Date of } \\
\text { Accident }\end{array}$ & $\begin{array}{c}\text { Accident } \\
\text { Description }\end{array}$ & $\begin{array}{c}\text { No. } \\
\text { of } \\
\text { Vhe } 1\end{array}$ & $\begin{array}{l}\text { Acc. } \\
\text { vel. } \\
\text { (mph) }\end{array}$ & $\begin{array}{c}\text { Fall } \\
\text { ht. } \\
(f t .)\end{array}$ & $\begin{array}{l}\text { Fire } \\
Y / N \\
\text { (dur) }\end{array}$ & $\begin{array}{l}\text { Object Struck } \\
\text { Description }\end{array}$ \\
\hline
\end{tabular}

\section{Alabama}

Huntsville NTSB 82-1 9/15/81 HtoS Col. $\quad 2 \quad 30$ NS Y(60M) Cargo Tank

\section{California}

Tracy

NTSB 76-1 3/9/75 HtoS Col.

250 NS N Gondola Car

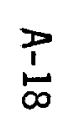

\section{Florida}

\begin{tabular}{|c|c|c|c|c|c|c|c|c|}
\hline Plant City & N/RHR-78-2 & $10 / 2 / 77$ & Train-Truck & 8 & 70 & NS & $Y(17 M)$ & Pickup Truck \\
\hline \multicolumn{9}{|l|}{ eorgia } \\
\hline Aragon & N/RHR-75-1 & $10 / 23 / 74$ & Train-Bus & 2 & 6 & 0 & $Y(N S)$ & Bus \\
\hline \multicolumn{9}{|l|}{ Minois } \\
\hline Beckemeyer & N/RHR-76-3 & $2 / 7 / 76$ & Train-Truck & 2 & NS & 0 & $N$ & Pickup Truck \\
\hline Elwood & N/RHR-76-2 & $11 / 19 / 75$ & Truck-Train & 2 & 82 & 0 & $N$ & Train \\
\hline Loda & N/RHR-71-1 & $1 / 24 / 70$ & Train-Truck & 2 & 79 & 0 & $Y(N S)$ & Tanker Truck \\
\hline
\end{tabular}


Table A.5

Rail-Highway Grade-Crossing Accidents

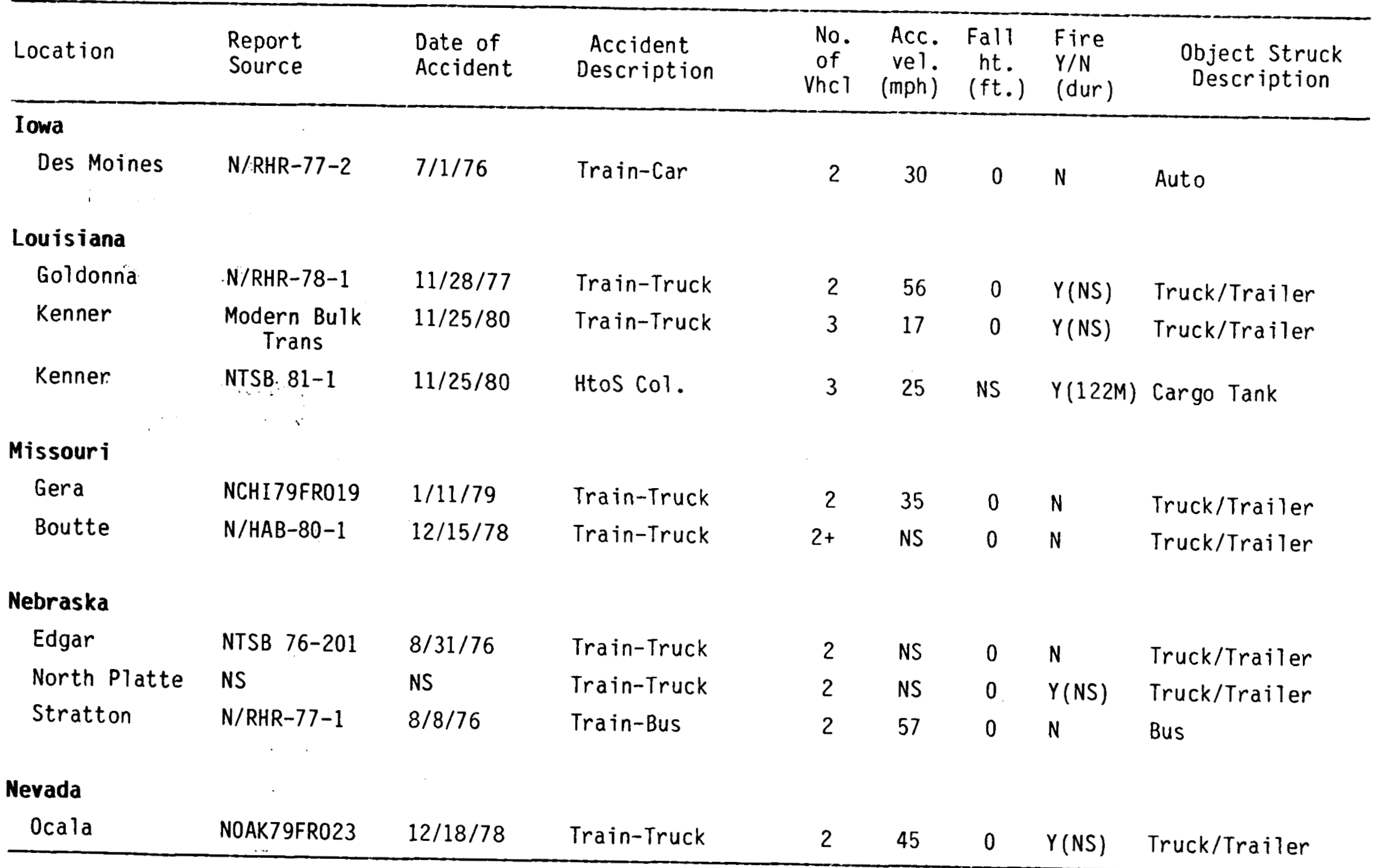


Table A.5

Rail-Highway Grade-Crossing Accidents

\begin{tabular}{|c|c|c|c|c|c|c|c|c|}
\hline Location & $\begin{array}{l}\text { Report } \\
\text { Source }\end{array}$ & $\begin{array}{l}\text { Date of } \\
\text { Accident }\end{array}$ & $\begin{array}{c}\text { Accident } \\
\text { Description }\end{array}$ & $\begin{array}{l}\text { No. } \\
\text { of } \\
\text { Vhc } 1\end{array}$ & $\begin{array}{l}\text { Acc. } \\
\text { vel. } \\
\text { (mph) }\end{array}$ & $\begin{array}{c}\text { Fall } \\
\text { ht. } \\
(\mathrm{ft.})\end{array}$ & $\begin{array}{l}\text { Fire } \\
\text { Y/N } \\
\text { (dur) }\end{array}$ & $\begin{array}{c}\text { Object Struck } \\
\text { Description }\end{array}$ \\
\hline \multicolumn{9}{|l|}{ New York } \\
\hline Congers & N/RHR-73-1 & $3 / 24 / 72$ & Train-Bus & 2 & 25 & 0 & N & Bus \\
\hline Mineola & NTSB 82-2 & $3 / 14 / 82$ & HtoS Col. & 2 & 65 & NS & $Y(20 M)$ & Van \\
\hline \multicolumn{9}{|l|}{ North Carolina } \\
\hline Sellers & NATL78FR011 & NS & Train-Truck & 2 & 79 & 0 & NS & Truck/Trailer \\
\hline \multicolumn{9}{|l|}{ Oklahoma } \\
\hline Collinsville & NTSB 72-1 & $4 / 5 / 71$ & HtoS Col. & 2 & 71 & NS & $N$ & Truck \\
\hline Marland & N/RHR $-77-3$ & $12 / 15 / 76$ & Train-Truck & 12 & 90 & 0 & $Y(N S)$ & Tanker Truck \\
\hline \multicolumn{9}{|l|}{ Oregon } \\
\hline Lafayette & NS & $9 / 8 / 76$ & Train-Bus & 2 & $50+$ & 0 & $N$ & Bus \\
\hline \multicolumn{9}{|l|}{ Pennsylvania } \\
\hline Southampton & NTSB $82-3$ & $1 / 2 / 82$ & Train-Truck & 3 & 20 & NS & $Y(135)$ & Trailer \\
\hline Yardley & $N / R H R-76-4$ & $6 / 5 / 75$ & Train-Truck & 3 & 63 & 0 & $N$ & Truck/Trailer \\
\hline
\end{tabular}


Table A.5

Rai1-Highway Grade-Crossing Accidents

\begin{tabular}{|c|c|c|c|c|c|c|c|c|}
\hline Location & $\begin{array}{l}\text { Report } \\
\text { Source }\end{array}$ & $\begin{array}{l}\text { Date of } \\
\text { Accident }\end{array}$ & $\begin{array}{c}\text { Accident } \\
\text { Description }\end{array}$ & $\begin{array}{c}\text { No. } \\
\text { of } \\
\text { Vhcl }\end{array}$ & $\begin{array}{l}\text { Acc. } \\
\text { ve } 1 . \\
\text { (mph) }\end{array}$ & $\begin{array}{c}\text { Fall } \\
\text { ht. } \\
\text { (ft.) }\end{array}$ & $\begin{array}{l}\text { Fire } \\
Y / N \\
\text { (dur) }\end{array}$ & $\begin{array}{c}\text { Object Struck } \\
\text { Description }\end{array}$ \\
\hline Virginia & & $\because$ & & & & & & \\
\hline $\begin{array}{c}\text { Tazewelt } \\
\ddots \\
\vdots\end{array}$ & NTSB $76-135$ & $\begin{array}{l}\text { NS } \\
:^{r} \vdots \vdots\end{array}$ & Train-Truck & 2 & 31 & 0 & $Y(N S)$ & Trailer \\
\hline West Virgina & & & & & & & & \\
\hline $\begin{array}{c}\text { Woodland } \\
\qquad \begin{array}{c}: \\
\vdots \\
\ddots\end{array}\end{array}$ & FRA $C-8-72$ & 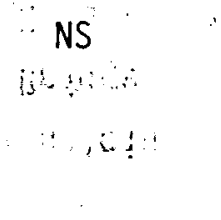 & Train-Vhcle & 2 & 40 & NS & NS & Earthmover \\
\hline$\because 1$ & & 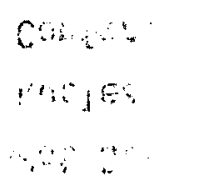 & & & & & & \\
\hline
\end{tabular}




\section{Report Source}

BMCS

CONF

DOT

DOTHS

$N / H A B$

N/HAR

NS

NUREG/CR

PATRAM

\section{Accident Description}

Bidg Col.

Brdg Ovtrn

$\mathrm{HtoH} \mathrm{Col}$.

HtoS Col.

HtoT Col.

Mltpl Col.

NS Trk. Fire

Ovtrn Col.

Trailer Sep.
Bureau of Motor Carrier Safety

\section{Conference}

Department of Transportation

Department of Transportation

National Transportation Safety Board, Highway Accident Brief

National Transportation Safety Board, Highway Accident Report

Not Stated

Nuclear Regulatory Commission Contractor Report

Conference on Packaging and Transportation of Radioactive Materials

Building Collision

Bridge Overturn

Head to Head Collision

Head to Side Collision

Head to Tail Collision

Multiple Collision

Not Stated Truck Fire

Overturn Collision

Trailer Separation 
Table A.6

Truck Accidents

\begin{tabular}{|c|c|c|c|c|c|c|c|c|}
\hline Location & $\begin{array}{l}\text { Report } \\
\text { Source }\end{array}$ & $\begin{array}{l}\text { Date of } \\
\text { Accident }\end{array}$ & $\begin{array}{l}\text { Accident } \\
\text { Description }\end{array}$ & $\begin{array}{l}\text { No. } \\
\text { of } \\
\text { Vhc } 1\end{array}$ & $\begin{array}{l}\text { Acc. } \\
\text { vel. } \\
\text { (mph) }\end{array}$ & $\begin{array}{l}\text { Fall } \\
\text { ht. } \\
\text { (ft.) }\end{array}$ & $\begin{array}{l}\text { Fire } \\
\text { Y/N } \\
\text { (dur) }\end{array}$ & $\begin{array}{l}\text { Object Struck } \\
\text { Description }\end{array}$ \\
\hline \multicolumn{9}{|l|}{ Arizona } \\
\hline Buckeye & $\mathrm{N} / \mathrm{HAB}-80-1$ & $11 / 15 / 78$ & $\mathrm{HtoH} \mathrm{Col}$. & 2 & NS & 0 & $N$ & Tractor Truck \\
\hline Gila Bend & BMCS 76-4 & NS & HtoH Col. & 6 & 80 & 0 & $Y(N S)$ & Car, Motorcycle \\
\hline \multicolumn{9}{|l|}{ Arkansas } \\
\hline Brisco & NS & $4 / 27 / 76$ & Overturn & 1 & 40 & 30 & $Y(N S)$ & Roadbed \\
\hline Camden & N/HAB-80-2 & $4 / 13 / 78$ & $\mathrm{HtoH} \mathrm{Col}$. & 2 & NS & 0 & $\mathrm{~N}$ & Pickup Truck \\
\hline Jasper & N/HAR-81-1 & $6 / 5 / 80$ & Explosion & 1 & 63 & 38 & $N$ & Hillside \\
\hline Little Rock & $N / H A B-80-1$ & $1 / 27 / 78$ & $\mathrm{HtoH} \mathrm{Col}$. & 3 & NS & 0 & $N$ & Truck/Trailer \\
\hline \multicolumn{9}{|l|}{ California } \\
\hline Coachella & N/HAR-80-6 & $4 / 23 / 80$ & $\mathrm{HtoH} \mathrm{Col}$. & 2 & 60 & NS & $N$ & Bus \\
\hline Coalinga & N/HAB- $80-1$ & $12 / 15 / 78$ & $\mathrm{HtoH} \mathrm{Col}$. & 12 & 47 & 0 & $N$ & Mitpl Cars \\
\hline Corona & N/HAR-75-7 & $2 / 28 / 75$ & Mltpl Col. & 84 & 50 & 0 & $Y(N S)$ & $\begin{array}{l}\text { M1tpl Cars, } \\
\text { Trucks }\end{array}$ \\
\hline $\begin{array}{l}\text { E1 Centro } \\
35 \mathrm{MI} \mathrm{W}\end{array}$ & N/HAR-75-6 & $3 / 8 / 74$ & $\mathrm{HtoH} \mathrm{Col}$. & 2 & 45 & NS & $N$ & Semi Trailer \\
\hline Lemoore & N/HAR-83-02 & $10 / 8 / 82$ & HtoH Col. & 3 & 55 & NS & $N$ & Van \\
\hline Los Angeles & NS & NS & Explosion & 6 & 0 & 0 & $Y(N S)$ & None \\
\hline
\end{tabular}


Table A.6

Truck Accidents

\begin{tabular}{|c|c|c|c|c|c|c|c|c|}
\hline Location & $\begin{array}{l}\text { Report } \\
\text { Source }\end{array}$ & $\begin{array}{l}\text { Date of } \\
\text { Accident }\end{array}$ & $\begin{array}{l}\text { Accident } \\
\text { Description }\end{array}$ & $\begin{array}{l}\text { No: } \\
\text { of } \\
\text { Vhc } 1\end{array}$ & $\begin{array}{l}\text { Acc. } \\
\text { vel. } \\
\text { (mph) }\end{array}$ & $\begin{array}{l}\text { Fall } \\
\text { ht. } \\
\text { (ft.) }\end{array}$ & $\begin{array}{l}\text { Fire } \\
Y / N \\
\text { (dur) }\end{array}$ & $\begin{array}{c}\text { Object Struck } \\
\text { Description }\end{array}$ \\
\hline Los Angeles & NS & NS & HtoH Col: & 6 & 55 & 0 & $Y(N S)$ & Truck/Trailer \\
\hline \multicolumn{9}{|c|}{ California (continued) } \\
\hline Los Angeles & N/HAR-80-5 & $3 / 3 / 80$ & Stos Col. & 3 & 45 & NS & $Y(55 M)$ & Tank Truck \\
\hline Martinez & N/HAR-77-2 & $5 / 21 / 76$ & Brdg Ovtrn & 1 & 35 & 22 & $\mathrm{~N}$ & Ground \\
\hline Oakland (near) & N/HAR-83-01 & $4 / 7 / 82$ & $\mathrm{HtoH} \mathrm{Col}$. & 3 & 55 & NS & $Y(162 M)$ & Car \\
\hline Ontario & NS & $11 / 4 / 74$ & Coll is ion & 1 & 50 & 0 & $Y(N S)$ & $\begin{array}{l}\text { Tree, Sign, } \\
\text { Steel, Concrete } \\
\text { Wall }\end{array}$ \\
\hline Sacramento & NS & NS & Overturn & 4 & NS & 0 & $Y(4 H)$ & Roadbed, Cars \\
\hline \multicolumn{9}{|l|}{ Sacramento } \\
\hline (near) & N/HAR-74-5 & $11 / 11 / 73$ & Collision & 1 & 67 & NS & $N$ & Concrete \\
\hline San Bernardino & N/HAR $-81-2$ & $11 / 10 / 80$ & $\mathrm{HtoH} \mathrm{Col}$. & 24 & 55 & NS & $N$ & Semi Trailer \\
\hline $\begin{array}{c}\text { San Francisco } \\
\text { Bay }\end{array}$ & San Jose News & $3 / 81$ & $\begin{array}{l}\text { Overpass } \\
\text { Run Off }\end{array}$ & 2 & 55 & 64 & $N$ & $\begin{array}{l}\text { Pickup } \\
\text { Truck, Ground }\end{array}$ \\
\hline Ventura & N/HAR-72-4 & $8 / 18 / 71$ & HtoH Col. & 13 & 60 & 0 & $Y(60 M)$ & Car \\
\hline Willow Creek & N/HAR-83-05 & $2 / 24 / 83$ & Skid & 2 & 38 & NS & $N$ & Bus \\
\hline Winterhaven & BMCS 79-2 & $4 / 4 / 79$ & Collision & 2 & NS & 0 & $Y(N S)$ & Parked Car \\
\hline \multicolumn{9}{|l|}{ Colorado } \\
\hline Canon City & N/HAR-82-3 & $11 / 14 / 81$ & HtoS Col. & 3 & 56 & NS & $Y(170 M)$ & Tractor \\
\hline
\end{tabular}


Table A. 6

Truck Accidents

\begin{tabular}{|c|c|c|c|c|c|c|c|c|}
\hline Location & $\begin{array}{l}\text { Report } \\
\text { Source }\end{array}$ & $\begin{array}{l}\text { Date of } \\
\text { Accident }\end{array}$ & $\begin{array}{l}\text { Accident } \\
\text { Description }\end{array}$ & $\begin{array}{l}\text { No. } \\
\text { of } \\
V h c l\end{array}$ & $\begin{array}{l}\text { Acc. } \\
\text { vel } \\
\text { (mph) }\end{array}$ & $\begin{array}{c}\text { Fal1 } \\
\text { ht. } \\
(\mathrm{ft.})\end{array}$ & $\begin{array}{l}\text { Fire } \\
Y / N \\
\text { (dur) }\end{array}$ & $\begin{array}{l}\text { Object Struck } \\
\text { Description }\end{array}$ \\
\hline Fleming & NS: & $9 / 29 / 77$ & HtoH Col. & 2 & 110 & 0 & $Y(N S)$ & Truck/Trailer \\
\hline \multicolumn{9}{|c|}{ Colorado (cont inued) } \\
\hline Golden & $\mathrm{NS}: \because$ & $6 / 10 / 74$ & Collision & 1 & 35 & 0 & $Y(5 H)$ & Rock Wall \\
\hline Golden & BMCS 8-186 & NS & Overturn & 1 & 95 & 30 & NS & $\begin{array}{l}\text { Roadbed, } \\
\text { Guardrai } 1\end{array}$ \\
\hline Kit Carson & BMC S: 8-097. & NS & HtoH Col: & 2 & 120 & NS & $Y(N S)$ & Truck/Trailer \\
\hline Kit Carson & BMCS :8-089 & NS & $\mathrm{HtoH} \mathrm{Col}$. & 2 & 110 & NS & $Y(N S)$ & Truck/Trailer \\
\hline Silverthorne & BMCS : 8-0.28 & NS & Collision & 2 & 55 & 15 & $Y(N S)$ & Guardrail \\
\hline$i \because$ & 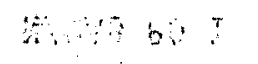 & $\therefore$ & & & & & & \\
\hline \multicolumn{2}{|c|}{ District of Columbia } & $\because$ & & & & & & \\
\hline Washington & $\begin{array}{c}\text { BMCS } 76-2 \\
\because \quad \because\end{array}$ & NS & Mltpl Col. & 2 & NS & NS & NS & Car \\
\hline Florida & $\cdots \cdots \cdots$ & & & & & & & \\
\hline Gretna & $N / H A R-72-3$ & $8 / 8 / 71$ & $\mathrm{HtOH} \mathrm{Col}$. & 2 & 50 & 2 & N & Car \\
\hline Homes tead & BMCS 7-178 & NS & HtoS Col. & 2 & 51 & NS & $Y(N S)$ & Truck/Trailer \\
\hline $\begin{array}{l}\text { Ocala } \\
\quad: \quad\end{array}$ & $\begin{array}{l}\text { N/HAR-83-04 } \\
\therefore .\end{array}$ & $2 / 28 / 83$ & HtoT/HtoS & 22 & $55+$ & NS & $Y(120 M)$ & Semi \\
\hline Georgia & $\cdots$ & & & & & & & \\
\hline $\begin{array}{l}\text { Atlanta } \\
\text { Atlanta }\end{array}$ & $\begin{array}{c}\text { N/HAR }-78-5 \\
\therefore\end{array}$ & $6 / 20 / 77$ & $\mathrm{HtoH} \mathrm{Col}$. & 7 & 45 & 0 & $\mathrm{~N}$ & Cars, Truck \\
\hline
\end{tabular}


Table A.6

Truck Accidents

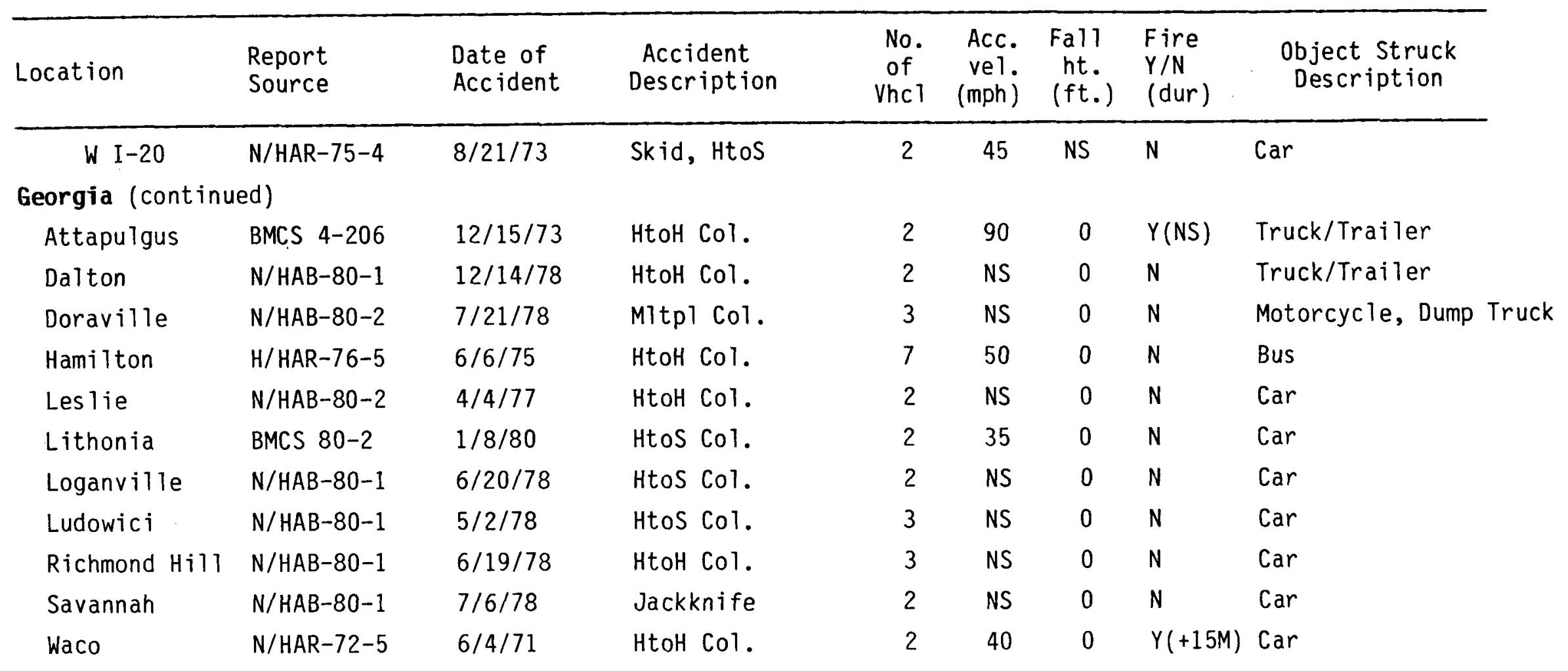

\section{Illinois}

$\begin{array}{lclllllll}\text { Gibson City } & \begin{array}{c}5 \text { th PATRAM } \\ \text { pg } 804-806\end{array} & \text { NS } & \text { Jackknife } & 1 & \text { NS } & \text { NS } & \text { NS } & \text { Roadbed } \\ \text { Rosecrans } & \text { BMCS } 5-030 & 4 / 29 / 76 & \text { Collision } & 1 & 55 & 0 & Y(N S) & \text { Bridge Barrier }\end{array}$


Table A. 6

Truck Accidents

\begin{tabular}{|c|c|c|c|c|c|c|c|c|}
\hline Location & $\begin{array}{l}\text { Report } \\
\text { Source }\end{array}$ & $\begin{array}{l}\text { Date of } \\
\text { Accident }\end{array}$ & $\begin{array}{l}\text { Accident } \\
\text { Description }\end{array}$ & $\begin{array}{l}\text { No. } \\
\text { of } \\
\text { Vhcl }\end{array}$ & $\begin{array}{l}\text { Acc. } \\
\text { vel } \\
\text { (mph) }\end{array}$ & $\begin{array}{c}\text { Fall } \\
\text { ht. } \\
(\mathrm{ft.})\end{array}$ & $\begin{array}{l}\text { Fire } \\
Y / N \\
\text { (dur) }\end{array}$ & $\begin{array}{l}\text { Object Struck } \\
\text { Description }\end{array}$ \\
\hline \multicolumn{9}{|l|}{ Indiana } \\
\hline Chesterton & NS & NS & Jackknife & 1 & 55 & 20 & $N$ & Guardrail \\
\hline Indianapolis & BMCS 75-5 & $6 / 13 / 75$ & Overturn & 1 & 50 & 18 & NS & Roadbed \\
\hline \multicolumn{9}{|l|}{ Iowa } \\
\hline Winthrop & $\mathrm{N} / \mathrm{HAB}-80-1$ & $5 / 2 / 78$ & Overturn & 1 & NS & 0 & $N$ & Roadbed \\
\hline \multicolumn{9}{|l|}{ Kansas } \\
\hline Kansas City & BMCS $7-0.64$ & $8 / 6 / 76$ & Cargo Loss & 1 & NS & 0 & $Y(N S)$ & Roadbed \\
\hline Leon & $N / H A B-80-2$ & $5 / 15 / 78$ & HtoH Col. & 3 & NS & 0 & $Y(N S)$ & Car \\
\hline Mayetta & BMCS 80-1 & $1 / 6 / 80$ & HtoH Col. & 2 & 50 & 0 & $Y(N S)$ & Pickup Truck \\
\hline Wichita & NUREG/CR-0992 & NS & Overturn & 1 & NS & NS & NS & Roadbed \\
\hline \multicolumn{9}{|l|}{ Kentucky } \\
\hline Beattyville & N/HAR-78-4 & $9 / 24 / 77$ & Runaway & 17 & 36 & 0 & $Y(5 H)$ & Roadbed \\
\hline Carroll City & DOTHS602826 & $8 / 75$ & HtoH Col. & 3 & 60 & 0 & $Y(105 M)$ & Car/Trailer \\
\hline
\end{tabular}


Table A. 6

Truck Accidents

\begin{tabular}{|c|c|c|c|c|c|c|c|c|}
\hline Location & $\begin{array}{l}\text { Report } \\
\text { Source }\end{array}$ & $\begin{array}{l}\text { Date of } \\
\text { Accident }\end{array}$ & $\begin{array}{l}\text { Accident } \\
\text { Description }\end{array}$ & $\begin{array}{l}\text { No. } \\
\text { of } \\
\text { Vhe } 1\end{array}$ & $\begin{array}{l}\text { Acc. } \\
\text { vel. } \\
\text { (mph) }\end{array}$ & $\begin{array}{c}\text { Fall } \\
\text { ht. } \\
(\mathrm{ft.})\end{array}$ & $\begin{array}{l}\text { Fire } \\
Y / N \\
\text { (dur) }\end{array}$ & $\begin{array}{l}\text { Object Struck } \\
\text { Description }\end{array}$ \\
\hline \multicolumn{9}{|l|}{ Louisiana } \\
\hline Baton Rouge & NS & NS & Overturn & 1 & NS & 0 & $Y(N S)$ & Roadbed \\
\hline Lake Charles & N/HAR-82-4 & $8 / 27 / 81$ & Skid & 26 & $30+$ & NS & $\mathrm{N}$ & Semi Trailer \\
\hline Ramah & $N / H A B-80-2$ & $12 / 16 / 78$ & Mltpl Col. & 4 & NS & 0 & $Y(N S)$ & Bridge Column \\
\hline \multicolumn{9}{|l|}{ Maryland } \\
\hline Bethesda & BMCS 78-2 & $3 / 14 / 78$ & Mltpl Col. & 3 & 70 & 40 & $\mathrm{~N}$ & Car \\
\hline Frostburg & $N / H A R-81-3$ & $2 / 18 / 81$ & Htos Col. & 17 & $50+$ & NS & $N$ & Truck \\
\hline Hagerstown & $N / H A B-80-1$ & $1 / 30 / 79$ & HtoH Col.. & 2 & NS & 0 & $N$ & Truck/Trailer \\
\hline N. Carrollton & N/HAR-71-9 & $6 / 19 / 70$ & Skid, HtoT & 2 & NS & NS & $N$ & Truck \\
\hline \multicolumn{9}{|l|}{ Massachusetts } \\
\hline Belcherstown & NS & NS & Collision & 1 & 60 & 25 & $N$ & Utility Pole \\
\hline Braintree & N/HAR-74-4 & $10 / 18 / 73$ & Overturn & 1 & 55 & 0 & $Y(N S)$ & Roadbed \\
\hline \multicolumn{9}{|l|}{ Michigan } \\
\hline Detroit & NS & $2 / 7 / 77$ & Collision & 1 & 45 & 30 & $Y(N S)$ & Bridge Barrier \\
\hline Flint & BMCS 5-076 & $8 / 19 / 76$ & Collision & $1+$ & NS & 20 & $Y(N S)$ & $\begin{array}{l}\text { Bridge Rail, } \\
\text { Roadbed }\end{array}$ \\
\hline
\end{tabular}


Table A.6

Truck Accidents

\begin{tabular}{|c|c|c|c|c|c|c|c|c|}
\hline Location : & $\begin{array}{l}\text { Report } \\
\text { Source }\end{array}$ & $\begin{array}{l}\text { Date of } \\
\text { Accident }\end{array}$ & $\begin{array}{l}\text { Accident } \\
\text { Description }\end{array}$ & $\begin{array}{l}\text { No. } \\
\text { of } \\
\text { Vhcl }\end{array}$ & $\begin{array}{l}\text { Acc. } \\
\text { vel. } \\
\text { (mph) }\end{array}$ & $\begin{array}{l}\text { Fall } \\
\text { ht. } \\
\text { (ft.) }\end{array}$ & $\begin{array}{l}\text { Fire } \\
Y / N \\
\text { (dur) }\end{array}$ & $\begin{array}{l}\text { Object Struck } \\
\text { Description }\end{array}$ \\
\hline \multicolumn{9}{|l|}{ Minnesota } \\
\hline $\begin{array}{c}\text { Floodwood } \\
\end{array}$ & BMCS 5-169 & NS & $\mathrm{HtoH} \mathrm{Col}$. & 3 & 105 & 0 & NS & Truck/Trailer \\
\hline \multicolumn{9}{|l|}{ Mississippi } \\
\hline Waynesboro & N/HAR-82-2 & $10 / 12 / 81$ & HtoH Col. & 3 & 35 & NS & $N$ & Car/Pole \\
\hline \multicolumn{9}{|l|}{ Missouri } \\
\hline Fisk & BMCS 7-064 & NS & Coltision & 1 & 55 & 45 & NS & Bridge, River \\
\hline Keytesville & NS & $4 / 7 / 77$ & Collision & 1 & 55 & 30 & $N$ & Bridge Barrier \\
\hline Kansas City & $N / H A B-80-2$ & $7 / 13 / 77$ & Collision & 1 & 55 & 0 & $N$ & Bridge Column \\
\hline St. Louis & N/HAR-79-3 & $9 / 25 / 77$ & HtoH Col. & 2 & NS & 0 & $N$ & Car \\
\hline \multicolumn{9}{|l|}{ North Carolina } \\
\hline Hertford & NS & $1 / 10 / 78$ & Explosion & 2 & NS & 0 & $Y(N S)$ & NS \\
\hline Marion & N/HAR-78-6 & $1 / 25 / 78$ & $\mathrm{HtoH} \mathrm{Col}$. & 2 & 70 & 0 & $\mathrm{~N}$ & Pickup Truck \\
\hline Morganton & NS & $4 / 27 / 78$ & $\mathrm{HtoH} \mathrm{Col}$. & 2 & 75 & 0 & $N$ & Truck \\
\hline \multicolumn{9}{|l|}{ North Dakota } \\
\hline Freeman & BMCS $80-3$ & $3 / 12 / 80$ & HtoH Col. & 4 & 40 & 0 & $Y(N S)$ & Cars \\
\hline
\end{tabular}


Table A.6

Truck Accidents

\begin{tabular}{|c|c|c|c|c|c|c|c|c|}
\hline Location & $\begin{array}{l}\text { Report } \\
\text { Source }\end{array}$ & $\begin{array}{l}\text { Date of } \\
\text { Accident }\end{array}$ & $\begin{array}{l}\text { Accident } \\
\text { Description }\end{array}$ & $\begin{array}{l}\text { No. } \\
\text { of } \\
\text { Vhc } 1\end{array}$ & $\begin{array}{l}\text { Acc. } \\
\text { vel. } \\
\text { (mph) }\end{array}$ & $\begin{array}{c}\text { Fall } \\
\text { ht. } \\
(\mathrm{ft.})\end{array}$ & $\begin{array}{l}\text { Fire } \\
Y / N \\
\text { (dur) }\end{array}$ & $\begin{array}{l}\text { Object Struck } \\
\text { Description }\end{array}$ \\
\hline \multicolumn{9}{|l|}{ New Jersey } \\
\hline Bordentown & $N / H A R-75-3$ & $10 / 19 / 73$ & Side Col. & 4 & 55 & 50 & $Y(N S)$ & Car \\
\hline Elizabethtown & NS & $9 / 27 / 77$ & Explosion & 1 & 0 & 0 & $Y(N S)$ & NS \\
\hline $\begin{array}{c}\text { Turnpike } \\
\text { Exit } 8\end{array}$ & N/HAR-73-4 & $10 / 17 / 73$ & Side Col. & 3 & $65+$ & 0 & $Y(30 M)$ & Guardrail \\
\hline \multicolumn{9}{|l|}{ New York } \\
\hline Alden & $N / H A B-80-1$ & $3 / 15 / 78$ & Collision & 4 & NS & 0 & $N$ & Car \\
\hline Brant & DOTHS801925 & $6 / 21 / 75$ & Collision & 1 & 55 & 35 & NS & Post, Roadbed \\
\hline Brooklyn & N/HAR-71-6 & $5 / 30 / 70$ & Explosion & 1 & 0 & 0 & $Y(N S)$ & NS \\
\hline Buffalo & DOTHS600979 & $3 / 19 / 71$ & $\mathrm{HtoH} \mathrm{Col}$. & 2 & 55 & 0 & NS & Truck/Trailer \\
\hline Buffalo & DOTHS600974 & $3 / 24 / 71$ & overturn & 1 & 60 & NS & NS & Roadbed \\
\hline Hamburg & DOTHS601762 & $4 / 10 / 72$ & Overturn & 1 & 40 & NS & NS & Roadbed \\
\hline Locke & NS & NS & Jackknife & 21 & NS & 0 & $Y(N S)$ & Building \\
\hline Moreau & $N / H A B-80-1$ & $8 / 13 / 78$ & HtoH Col. & 2 & NS & 0 & $N$ & Truck/Trailer \\
\hline \multicolumn{9}{|l|}{ Ohio } \\
\hline Ashtabula & Newscast & $4 / 1 / 81$ & Overturn & 1 & NS & NS & NS & Roadbed \\
\hline Valley View & N/HAR-77-3 & $8 / 20 / 76$ & Mltpl Col. & 11 & 50 & 0 & $Y(N S)$ & Mltpl Cars \\
\hline
\end{tabular}


Table A. 6

Truck Accidents

\begin{tabular}{|c|c|c|c|c|c|c|c|c|}
\hline Location & $\begin{array}{l}\text { Report } \\
\text { Source }\end{array}$ & $\begin{array}{l}\text { Date of } \\
\text { Accident }\end{array}$ & $\begin{array}{l}\text { Accident } \\
\text { Description }\end{array}$ & $\begin{array}{c}\text { No. } \\
\text { of } \\
\text { Vhc } 1\end{array}$ & $\begin{array}{l}\text { Acc. } \\
\text { vel } \\
\text { (mph) }\end{array}$ & $\begin{array}{c}\text { Fall } \\
\text { ht. } \\
(\mathrm{ft.})\end{array}$ & $\begin{array}{l}\text { Fire } \\
Y / N \\
\text { (dur) }\end{array}$ & $\begin{array}{c}\text { Object Struck } \\
\text { Description }\end{array}$ \\
\hline \multicolumn{9}{|l|}{ Oklahoma } \\
\hline El Reno & BMCS 6-606 & NS & $\mathrm{HtoH} \mathrm{Col}$. & 2 & 50 & 31 & $N$ & Truck/Trailer \\
\hline Stroud & BMCS 6-046 & NS & Collision & 1 & 45 & 25 & $Y(N S)$ & Guardrait \\
\hline \multicolumn{9}{|l|}{ Oregon } \\
\hline Portland & DOT $72-5$ & $11 / 18 / 72$ & Side Col. & 1 & NS & 0 & $N$ & Concrete Wal1 \\
\hline \multicolumn{9}{|l|}{ Pennsylvania } \\
\hline Clarion & BMCS $69-5$ & NS & Collision & 1 & 20 & 13 & $N$ & Bridge \\
\hline Fulton County & $N / H A B-80-1$ & $2 / 22 / 79$ & Overturn & 1 & NS & 0 & $N$ & Ground \\
\hline Indiana & N/HAR-80-3 & $9 / 22 / 79$ & HtoH Col. & 2 & 70 & NS & $\mathrm{N}$ & Car \\
\hline Lamar & N/HAB-80-1 & $2 / 7 / 79$ & Run off Rd & 2 & NS & 0 & $\mathrm{~N}$ & Guardrait \\
\hline Lancaster Cnty & $\mathrm{N} / \mathrm{HAR}-72-1$ & $2 / 6 / 72$ & Collision & 1 & 55 & NS & $\mathrm{N}$ & Guardrail \\
\hline Mt. Pleasant & $N / H A B-80-1$ & $2 / 14 / 79$ & Trailer Sep. & 2 & NS & 0 & $N$ & Car \\
\hline N. Cumberland & BMCS 3-208 & NS & Overturn & 2 & 55 & 0 & $\mathrm{~N}$ & Roadbed \\
\hline Washington & NS & NS & Collision & 1 & 50 & 0 & $Y(3 H)$ & Guardrai 1 \\
\hline Washington & NS & NS & Overturn & 7 & 50 & 0 & N & Roadbed \\
\hline Warfordsburg & N/HAB- $80-1$ & $5 / 5 / 79$ & Overturn & 1 & 70 & 0 & $N$ & Roadbed \\
\hline
\end{tabular}


Table A. 6

Truck Accidents

\begin{tabular}{|c|c|c|c|c|c|c|c|c|}
\hline Location & $\begin{array}{l}\text { Report } \\
\text { Source }\end{array}$ & $\begin{array}{l}\text { Date of } \\
\text { Accident }\end{array}$ & $\begin{array}{l}\text { Accident } \\
\text { Description }\end{array}$ & $\begin{array}{l}\text { No. } \\
\text { of } \\
\text { Vhci } 1\end{array}$ & $\begin{array}{l}\text { Acc. } \\
\text { vel. } \\
\text { (mph) }\end{array}$ & $\begin{array}{l}\text { Fall } \\
\text { ht. } \\
\text { (ft.) }\end{array}$ & $\begin{array}{l}\text { Fire } \\
\text { Y/N } \\
\text { (dur) }\end{array}$ & $\begin{array}{l}\text { Object Struck } \\
\text { Description }\end{array}$ \\
\hline
\end{tabular}

\section{Rhode İs land}

West Greeniwich $N / H A B-80-1$

1/26/79 Bidg Col.

1 NS 0 N Building

\section{Tennessee}

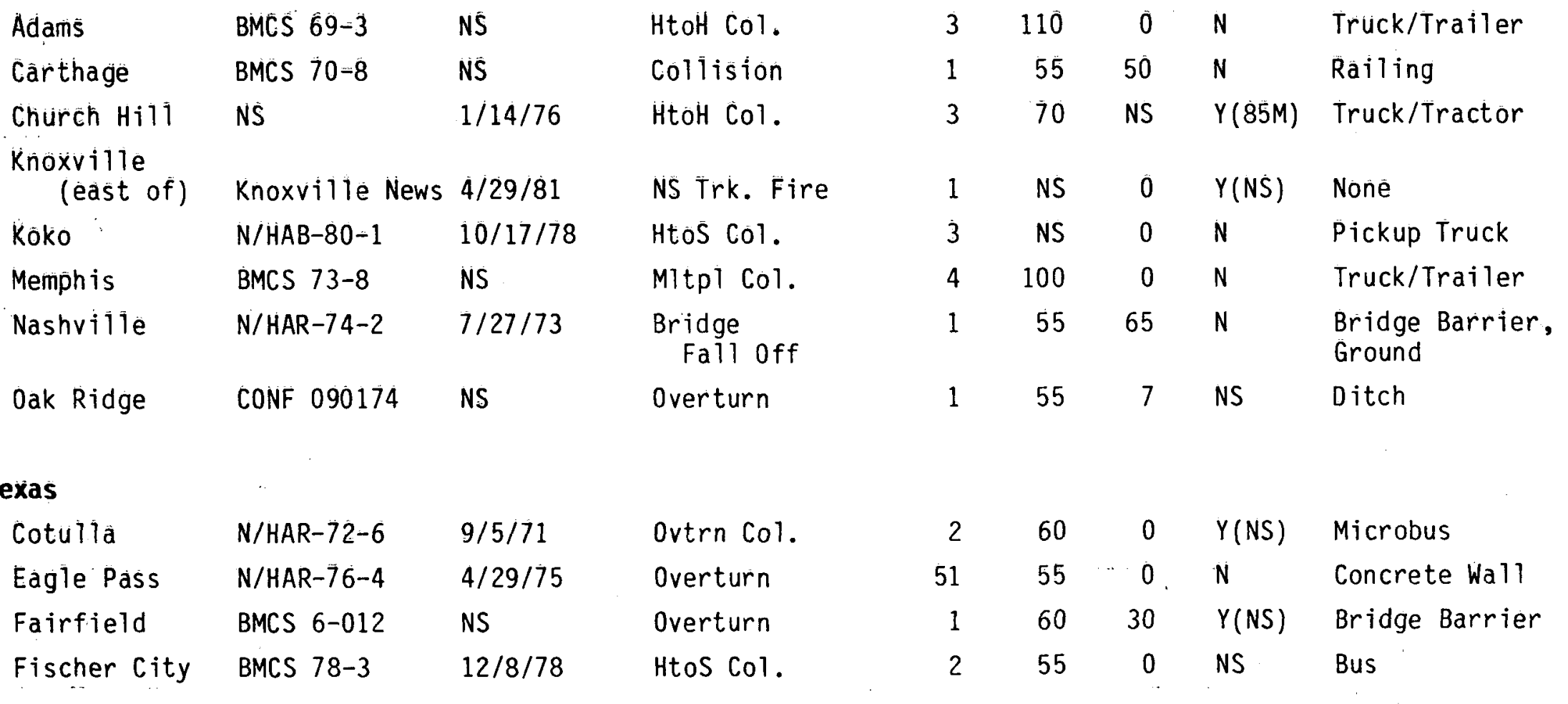


Table A. 6

Truck Accidents

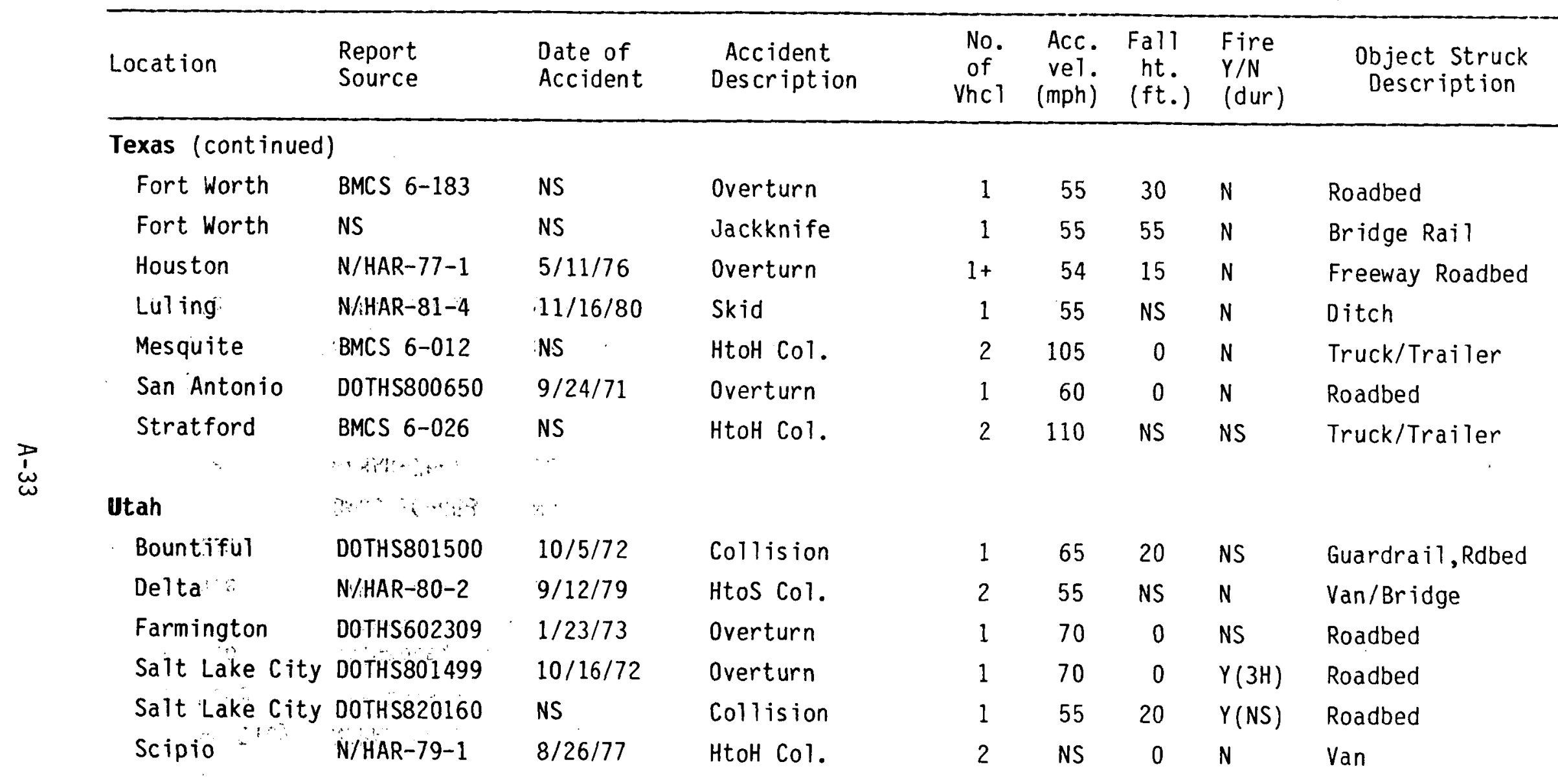


Table A. 6

Truck Accidents

\begin{tabular}{|c|c|c|c|c|c|c|c|c|}
\hline Location & $\begin{array}{l}\text { Report } \\
\text { Source }\end{array}$ & $\begin{array}{l}\text { Date of } \\
\text { Accident }\end{array}$ & $\begin{array}{l}\text { Accident } \\
\text { Description }\end{array}$ & $\begin{array}{c}\text { No. } \\
\text { of } \\
\text { Vhel }\end{array}$ & $\begin{array}{l}\text { Acc. } \\
\text { vel } \\
\text { (mph) }\end{array}$ & $\begin{array}{c}\text { Fall } \\
\text { ht. } \\
(f t .)\end{array}$ & $\begin{array}{l}\text { Fire } \\
Y / N \\
\text { (dur) }\end{array}$ & $\begin{array}{l}\text { Object Struck } \\
\text { Description }\end{array}$ \\
\hline \multicolumn{9}{|l|}{ Virginia } \\
\hline Hanover City & N/HAB-80-1 & $12 / 17 / 79$ & $\mathrm{HtoH} \mathrm{Col}$. & 2 & NS & 0 & $N$ & Car \\
\hline Lynchburg & H/HAR-73-3 & $3 / 9 / 72$ & Overturn & 1 & 25 & 0 & $Y(22 H)$ & Rock \\
\hline quantico & $\begin{array}{l}\text { Columbus, } \mathrm{OH} \\
\text { News }\end{array}$ & $2 / 19 / 81$ & $\begin{array}{l}\text { Bridge } \\
\quad \text { Run off }\end{array}$ & 1 & 55 & 80 & $N$ & $\begin{array}{l}\text { Brdg Under } \\
\text { Structure }\end{array}$ \\
\hline Triangle & N/HAR-81-6 & $2 / 18 / 81$ & Collision & 1 & 60 & 25 & $N$ & Guardrail \\
\hline \multicolumn{9}{|l|}{ Hashington } \\
\hline Pasco & BMCS 10-058 & NS & $\mathrm{HtoH} \mathrm{Col}$. & 4 & 110 & NS & NS & Truck/Trailer \\
\hline Seattle & N/HAR-76-7 & $12 / 4 / 75$ & Jackknife & 35 & 52 & 0 & $N$ & Support Column \\
\hline \multicolumn{9}{|l|}{ Hyoming } \\
\hline Baggs & NS & $8 / 2 / 74$ & Side Col. & 2 & NS & 0 & $Y(N S)$ & NS \\
\hline Laramie & N/HAR-80-1 & $8 / 22 / 79$ & $\mathrm{HtoH} \mathrm{Col}$. & 3 & 68 & 0 & $N$ & House, Vehicle \\
\hline
\end{tabular}


Table A.7 Legend

Train Accidents

\section{Report Source}

\begin{tabular}{|c|c|}
\hline ASME & American Society of Mechanical Engineers \\
\hline DOT & Department of Transportation \\
\hline FRA & Federal Railroad Administration \\
\hline ICC & Interstate Commerce Commission \\
\hline $\begin{array}{l}\text { NATL, year, report \# } \\
\text { NCHI, year, report \# } \\
\text { NDCA, year, report \# }\end{array}$ & $\begin{array}{l}\text { Department of Transportation, Federal Railroad Administration, Atlanta Office } \\
\text { Department of Transportation, Federal Railroad Administration, Chicago Office } \\
\text { Department of Transportation, Federal Railroad Administration, Washington D.C. } \\
\text { Office }\end{array}$ \\
\hline $\begin{array}{l}\text { NDEN, year, report \# } \\
\text { NFTW, year, report \# }\end{array}$ & $\begin{array}{l}\text { Department of Transportation, Federal Railroad Administration, Denver Office } \\
\text { Department of Transportation, Federal Railroad Administration, Fort Worth } \\
\text { Office }\end{array}$ \\
\hline $\mathrm{N} / \mathrm{HZM}$ & National Transportation Safety Board, Hazardous Material Accident Report \\
\hline NMKC, year, report \# & $\begin{array}{l}\text { Department of Transportation, Federal Railroad Administration, Kansas City } \\
\text { Office }\end{array}$ \\
\hline NNYC, year, report \# & $\begin{array}{l}\text { Department of Transportation, Federal Railroad Administration, New York City } \\
\text { Office }\end{array}$ \\
\hline $\begin{array}{l}\text { NOAK, year, report \# } \\
\text { N/RAR }\end{array}$ & $\begin{array}{l}\text { Department of Transportation, Federal Railroad Administration, Oakland Office } \\
\text { National Transportation Safety Board, Railroad Accident Report }\end{array}$ \\
\hline IS & Not Stated \\
\hline
\end{tabular}


Table A.7 Legend Continued

Tra in Accidents

\section{Report Number}

NSEA, year

\section{Accident Description}

Brdg Col.

Brdg Fail

Drl Col.

$\mathrm{HtoH}$ Col.

HtoS Col.

HtoT Col.

Int. Fire
Department of Transportation, Federal Railroad Administration, Seattle Office
Bridge Collision

Bridge Failure

Derail Collision

Head to Head Collision

Head to Side Collision

Head to Tail Collision

Internal Fire 
Table A.7

Train Accidents

\begin{tabular}{|c|c|c|c|c|c|c|c|c|}
\hline Location & $\begin{array}{l}\text { Report } \\
\text { Source }\end{array}$ & $\begin{array}{l}\text { Date of } \\
\text { Accident }\end{array}$ & $\begin{array}{l}\text { Accident } \\
\text { Description }\end{array}$ & $\begin{array}{l}\text { No. } \\
\text { of } \\
\text { Vhc1 }\end{array}$ & $\begin{array}{l}\text { Acc. } \\
\text { ve 1. } \\
\text { (mph) }\end{array}$ & $\begin{array}{c}\text { Fall } \\
\text { ht. } \\
\text { (ft.) }\end{array}$ & $\begin{array}{l}\text { Fire } \\
Y / N \\
\text { (dur) }\end{array}$ & $\begin{array}{l}\text { Object Struck } \\
\text { Description }\end{array}$ \\
\hline
\end{tabular}

Alabama .

\begin{tabular}{|c|c|c|c|c|c|c|c|c|}
\hline Florence & N/RAR-79-2 & $9 / 18 / 78$ & $\mathrm{HtoH} \mathrm{Col}$. & $2 T$ & 15 & 12 & N & Train \\
\hline Hunter & NATL78FR018 & $1 / 19 / 79$ & Brdg Col. & 72 & 8 & 75 & N & Bridge \\
\hline Muscle Shoals & NATL79FR001 & $10 / 8 / 78$ & $\mathrm{HtoH} \mathrm{Co} 1$. & $2 \mathrm{~T}$ & NS & 0 & N & Train \\
\hline North Castle & N/RAR-77-9 & $1 / 16 / 77$ & Derail & 22 & 43 & 21 & N & RR Bed, RR Car \\
\hline
\end{tabular}

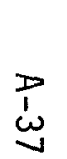

\section{Alaska}

Hurricane
Talkeetna

$$
\therefore ;
$$

\section{Arizona}

\section{Benson}

Benton

Dequeen

Raso

Rone

\begin{tabular}{lll} 
N/RAR-75-2 & $5 / 24 / 73$ & Explosion \\
NFT-W79FR018 & $12 / 25 / 78$ & Derail \\
\hdashline & & \\
NFTW79FR020 & $1 / 13 / 79$ & Derail \\
NOAK79FR017 & $12 / 10 / 78$ & Derail \\
NFTW79FR014 & $12 / 4 / 78$ & Derail
\end{tabular}

$12 / 4 / 78$
HtoH Col.

Derail

$\begin{array}{ll}\text { N/RAR-76-3 } & 7 / 5 / 75 \\ \text { NSEA77FR005 } & 12 / 1 / 76\end{array}$

$2 \mathrm{~T} \quad 40$

71 NS

$\begin{array}{rr}0 & N \\ 25 & N\end{array}$

Train

RR Bed, RR Car

$\begin{array}{rrrll}12 & 45 & 0 & Y(8 H) & \text { NS } \\ 137 & 45 & 23 & Y(3 H) & \begin{array}{l}\text { Bridge, RR Cars, } \\ \text { River } \\ 105\end{array} \\ 25 & 20 & \text { N } & \text { RR Bed, RR Car } \\ \text { NS } & 40 & 0 & \text { N } & \text { RR Bed, RR Car } \\ 125 & 15 & 14 & \text { N } & \text { RR Bed, RR Car }\end{array}$


Table A.7

Train Accidents

\begin{tabular}{|c|c|c|c|c|c|c|c|c|}
\hline Location & $\begin{array}{l}\text { Report } \\
\text { Source }\end{array}$ & $\begin{array}{l}\text { Date of } \\
\text { Accident }\end{array}$ & $\begin{array}{l}\text { Accident } \\
\text { Description }\end{array}$ & $\begin{array}{l}\text { No. } \\
\text { of } \\
\text { Vhc } 7\end{array}$ & $\begin{array}{l}\text { Acc. } \\
\text { vel. } \\
\text { (mph) }\end{array}$ & $\begin{array}{l}\text { Fall } \\
\text { ht. } \\
\text { (ft.) }\end{array}$ & $\begin{array}{l}\text { Fire } \\
Y / N \\
\text { (dur) }\end{array}$ & $\begin{array}{c}\text { Object Struck } \\
\text { Description }\end{array}$ \\
\hline
\end{tabular}

\begin{tabular}{|c|c|c|c|c|c|c|c|c|}
\hline \multicolumn{9}{|l|}{ Arkansas } \\
\hline Gilmore & NFTW79FR019 & $1 / 8 / 79$ & Derail & 97 & 55 & 0 & $N$ & RR Bed, RR Car \\
\hline Hartman & NFTW79FR008 & $2 / 27 / 77$ & Derail & 109 & 40 & 0 & $Y(200 M)$ & RR Bed, RR Car \\
\hline Lewisville & N/RAR-78-8 & $3 / 29 / 78$ & Derail & 47 & 35 & 0 & $Y(24 H)$ & RR Bed, RR Car \\
\hline Poping-0zark & NFTW79FR012 & $11 / 9 / 78$ & Derail & 131 & 38 & NS & $N$ & RR Bed, RR Car \\
\hline $\begin{array}{l}\text { Possum Grape } \\
\text { (near) }\end{array}$ & N/RAR-83-06 & $10 / 3 / 82$ & HtoS Col. & 2 & 50 & 30 & $Y(120)$ & Freight Car \\
\hline
\end{tabular}

\begin{tabular}{|c|c|c|c|c|c|c|c|c|}
\hline \multicolumn{9}{|c|}{ California } \\
\hline Andesite & N0AK79FR012 & $11 / 26 / 78$ & Derail & 70 & NS & 0 & $N$ & RR Bed, RR Car \\
\hline Bradley & NOAK79FR001 & $10 / 4 / 78$ & Derai 1 & 56 & 30 & 0 & $Y(5 D)$ & RR Bed, RR Car \\
\hline Hayward & N/RAR-80-10 & $4 / 9 / 80$ & Derai 1 & 1 & 52 & 30 & $Y(60 M)$ & RR Bed, RR Car \\
\hline Indio & N/RAR-74-1 & $6 / 25 / 73$ & HtoH Col. & $2 T$ & 60 & 0 & $Y(N S)$ & Train \\
\hline Kelso & N/RAR-81-7 & $11 / 17 / 80$ & $\mathrm{HtoH} \mathrm{Col}$. & 2 & 118 & NS & $N$ & Caboose \\
\hline Oroville & NOAK79FR011 & $11 / 20 / 78$ & Derai1 & 61 & 30 & 10 & $\mathrm{~N}$ & RR Bed, RR Car \\
\hline Pinole & N0AK79FR013 & $12 / 1 / 78$ & Derai 1 & 73 & 40 & 0 & $N$ & RR Bed, RR Car \\
\hline Roseville & DOT 4187 & $4 / 28 / 73$ & Explosion & 289 & 0 & 0 & $Y(32 H)$ & NS \\
\hline $\begin{array}{l}\text { San Francisco } \\
\text { Santa }\end{array}$ & N/RAR-79-5 & $1 / 17 / 79$ & Int. Fire & 2 & NS & 0 & $Y(2 H)$ & NS \\
\hline Margurita & N0AK79FR005 & $10 / 18 / 78$ & HtoH Col. & $2 T$ & 25 & 0 & $N$ & Train \\
\hline
\end{tabular}


Table A.7

Train Accidents

\begin{tabular}{|c|c|c|c|c|c|c|c|c|}
\hline Location & $\begin{array}{l}\text { Report } \\
\text { Source }\end{array}$ & $\begin{array}{l}\text { Date of } \\
\text { Accident }\end{array}$ & $\begin{array}{c}\text { Accident } \\
\text { Description }\end{array}$ & $\begin{array}{c}\text { No. } \\
\text { of } \\
\text { Vhcl }\end{array}$ & $\begin{array}{l}\text { Acc. } \\
\text { vel } \\
\text { (mph) }\end{array}$ & $\begin{array}{c}\text { Fall } \\
\text { ht. } \\
\text { (ft.) }\end{array}$ & $\begin{array}{l}\text { Fire } \\
\text { Y/N } \\
\text { (dur) }\end{array}$ & $\begin{array}{l}\text { Object Struck } \\
\text { Description }\end{array}$ \\
\hline
\end{tabular}

\begin{tabular}{|c|c|c|c|c|c|c|c|c|}
\hline \multicolumn{9}{|c|}{ California (continued) } \\
\hline Surf & N/RAR-81-1 & $5 / 22 / 81$ & Derail & 3 & 60 & NS & N & RR Bed, RR Car \\
\hline Therman & N/RAR-83-1 & $1 / 7 / 82$ & Derail & 61 & 57 & 0 & $N$ & RR Bed, RR Car \\
\hline Thousand Palms & $N / R A R-80-1$ & $7 / 24 / 79$ & HtoT Col. & $2 T$ & 20 & 0 & $Y(N S)$ & Train \\
\hline Vidal & NOAK79FR025 & $2 / 5 / 79$ & Derail & 78 & 45 & 15 & $\mathrm{~N}$ & RR Bed, RR Car \\
\hline
\end{tabular}

\section{Colorado}

$\begin{array}{llllllll}\text { Lambert NDEN76FR137 } & 7 / 9 / 76 & \text { Derail } & 38 & 60 & 5 & \mathrm{~N} & \text { RR Bed, RR Car }\end{array}$

\section{Connecticut}

$\begin{array}{lllllllll}\text { Darian } & \text { N/RAR-70-3 } & 8 / 20 / 69 & \text { HtoH Col. } & 2 \mathrm{~T} & 60 & 0 & \mathrm{~N} & \text { Train } \\ \text { North Canaan } & \text { N/RAR-77-4 } & 7 / 13 / 76 & \text { HtoH Col. } & 2 \mathrm{~T} & 20 & 0 & \mathrm{~N} & \text { Train } \\ \text { Sound View } & \text { N/RAR-72-1 } & 10 / 8 / 70 & \text { Drl. Col. } & 2 \mathrm{~T} & 60 & 0 & \mathrm{Y}(2.5 \mathrm{H}) \text { Train }\end{array}$

\section{Delaware}

Wilmingtion N/RAR-76-7 10/17/75 HtoH Col. $\quad 3$ T $25 \quad 0 \quad \mathrm{~N}$ Train


Table A.7

Train Accidents

\begin{tabular}{|c|c|c|c|c|c|c|c|c|}
\hline Location & $\begin{array}{l}\text { Report } \\
\text { Source }\end{array}$ & $\begin{array}{l}\text { Date of } \\
\text { Accident }\end{array}$ & $\begin{array}{c}\text { Accident } \\
\text { Description }\end{array}$ & $\begin{array}{l}\text { No. } \\
\text { of } \\
\text { Vhcl }\end{array}$ & $\begin{array}{l}\text { Acc. } \\
\text { vel. } \\
\text { (mph) }\end{array}$ & $\begin{array}{c}\text { Fall } \\
\text { ht. } \\
(\mathrm{ft.})\end{array}$ & $\begin{array}{l}\text { Fire } \\
Y / N \\
\text { (dur) }\end{array}$ & $\begin{array}{c}\text { Object Struck } \\
\text { Description }\end{array}$ \\
\hline \multicolumn{9}{|c|}{ District of Columbia } \\
\hline Washington & NDCA76FR151 & $7 / 18 / 76$ & Derail & 84 & 36 & 25 & $Y(N S)$ & $\begin{array}{l}\text { RR Bed, RR Car, } \\
\text { Highway }\end{array}$ \\
\hline Washington & N/RAR-82-6 & $1 / 13 / 82$ & Derail & 1 & 10 & NS & $\mathrm{N}$ & Wall \\
\hline \multicolumn{9}{|l|}{ Florida } \\
\hline Crestview & N/RAR-79-11 & $4 / 8 / 79$ & Derail & 119 & 35 & NS & $Y(60 H)$ & RR Bed, RR Car \\
\hline Loch loosa & N/RAR-81-9 & $5 / 26 / 81$ & Derail & 1 & 76 & NS & $N$ & RR Bed, RR Car \\
\hline Pensacola & $N / R A R-78-4$ & $11 / 9 / 77$ & Derail & 37 & 35 & 0 & $\mathrm{~N}$ & RR Bed, RR Car \\
\hline Westlake Wales & FRA $\quad 771-72$ & NS & Derail & 123 & 50 & NS & NS & RR Bed, RR Car \\
\hline Youngstown & N/RAR-78-8 & $2 / 26 / 78$ & Derail & 145 & 45 & 0 & $N$ & RR Bed, RR Car \\
\hline \multicolumn{9}{|l|}{ Georgia } \\
\hline Covington & NATL79FR025 & $2 / 19 / 79$ & Derail & 80 & 25 & 0 & $N$ & RR Bed, RR Car \\
\hline Pembroke & NATL79FR021 & $2 / 7 / 79$ & Derail & 134 & 31 & 5 & $N$ & RR Bed, RR Car \\
\hline Rupert & NATL76FR219 & $9 / 11 / 76$ & Derail & 108 & 50 & 0 & $N$ & RR Bed, RR Car \\
\hline Vinings & NATL79FR0 16 & $1 / 15 / 79$ & Derai 1 & 60 & 35 & 0 & $N$ & RR Bed, RR Car \\
\hline
\end{tabular}


Table A.7

Train Accidents

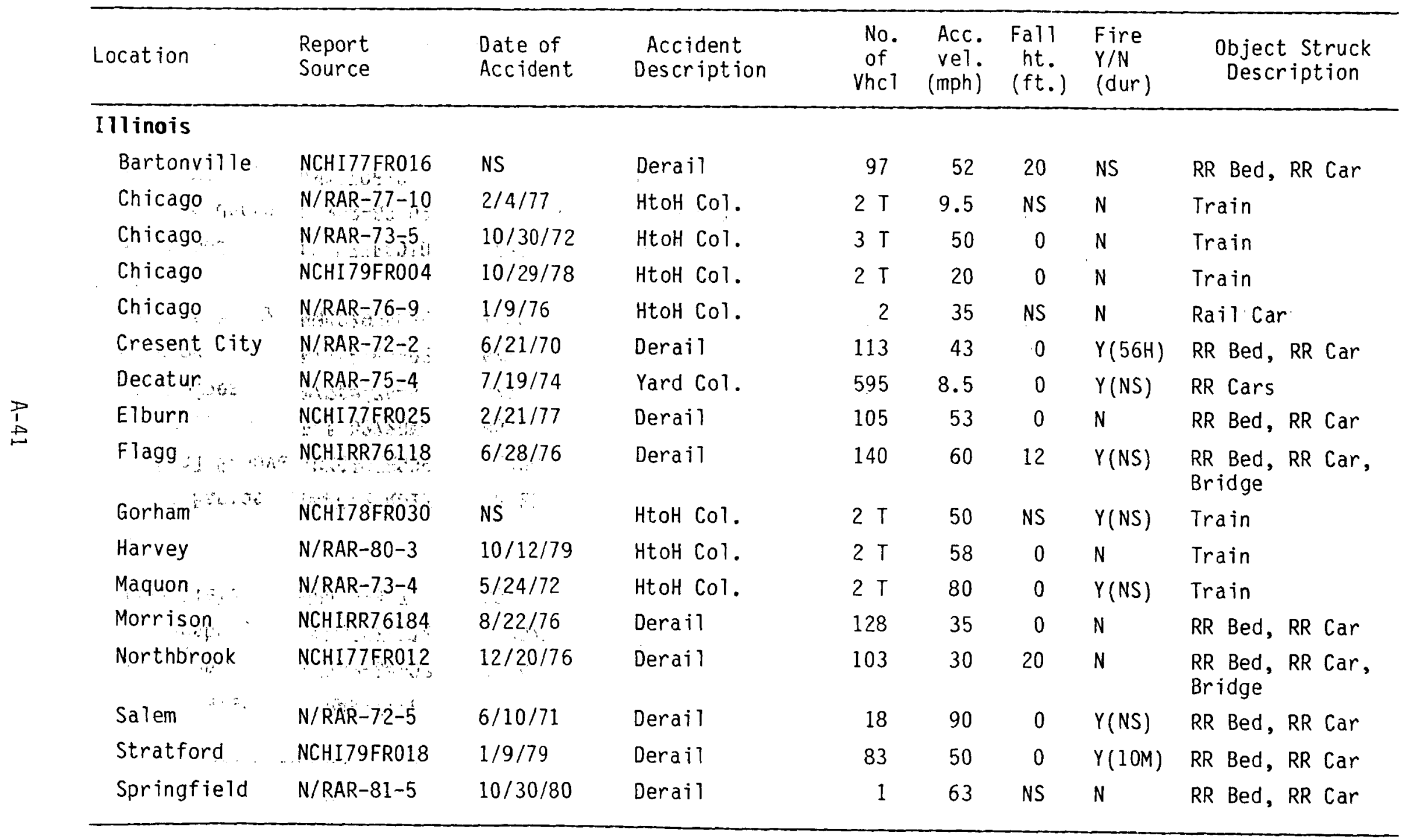


Table A.7

Train Accidents

\begin{tabular}{|c|c|c|c|c|c|c|c|c|}
\hline Location & $\begin{array}{l}\text { Report } \\
\text { Source }\end{array}$ & $\begin{array}{l}\text { Date of } \\
\text { Accident }\end{array}$ & $\begin{array}{l}\text { Accident } \\
\text { Description }\end{array}$ & $\begin{array}{l}\text { No. } \\
\text { of } \\
\text { Vhcl }\end{array}$ & $\begin{array}{l}\text { Acc. } \\
\text { vel. } \\
\text { (mph) }\end{array}$ & $\begin{array}{c}\text { Fall } \\
\text { ht. } \\
(\mathrm{ft.})\end{array}$ & $\begin{array}{l}\text { Fire } \\
Y / N \\
\text { (dur) }\end{array}$ & $\begin{array}{c}\text { Object Struck } \\
\text { Description }\end{array}$ \\
\hline \multicolumn{9}{|l|}{ Indiana } \\
\hline North Haven & N/RAR-77-6 & $10 / 19 / 76$ & $\mathrm{HtaH} \mathrm{Col}$. & $2 T$ & 20 & 0 & $Y(N S)$ & Train \\
\hline Sullivan & N/RAR-84-02 & $9 / 14 / 83$ & $\mathrm{HtoH} \mathrm{Col}$. & 2 & 35 & 0 & $N$ & Caboose \\
\hline Veedersburg & NCHI76FR112 & $6 / 25 / 76$ & Derail & 47 & 44 & NS & $N$ & RR Bed, RR Car \\
\hline Wheatfield & FRA $\quad B-8-72$ & NS & Derail & 109 & 40 & NS & $Y(2 H)$ & $\begin{array}{l}\text { RR Bed, RR Car, } \\
\text { Storage Tank }\end{array}$ \\
\hline \multicolumn{9}{|l|}{ Iowa } \\
\hline Cedar Rapids & NMKC79FR017 & $12 / 25 / 78$ & Derai1 & 13 & NS & 22 & $\mathbf{N}$ & River, Ice \\
\hline Central Groove & NMKC79FR009 & $11 / 28 / 78$ & Derail & 104 & 20 & 10 & $\mathrm{~N}$ & RR Bed, RR Car \\
\hline Cudley & FRA B272BN1 & NS & Derail & 93 & 60 & NS & $Y(N S)$ & RR Bed, RR Car \\
\hline Des Moines & N/RAR-76-8 & $9 / 1 / 75$ & Derail & 63 & 25 & 0 & $Y(4 D)$ & RR Bed, RR Car \\
\hline Emerson & N/RAR-83-02 & $6 / 15 / 82$ & Derail & 1 & 74 & NS & $N$ & RR Bed, RR Car \\
\hline Gordons Ferry & NMKC79FR030 & $1 / 28 / 79$ & Derail & 104 & 26 & 35 & $N$ & Miss. Rvr, RR \\
\hline \multicolumn{9}{|l|}{ Cars } \\
\hline Northwood & NMKC 77FR010 & $1 / 23 / 77$ & Derai1 & $104+$ & 40 & NS & $N$ & RR Bed, RR Car \\
\hline Pacific Jnctn & N/RAR-83-09 & $4 / 13 / 83$ & $\mathrm{HtoH} \mathrm{Col}$. & 2 & 47 & NS & $N$ & Caboose \\
\hline Woodburn & NMKC79FR023 & $1 / 12 / 79$ & Derail & 106 & 50 & 0 & N & RR Bed, RR Car \\
\hline
\end{tabular}


Table A.7

Train Accidents

\begin{tabular}{|c|c|c|c|c|c|c|c|c|}
\hline Location & $\begin{array}{l}\text { Report } \\
\text { Source }\end{array}$ & $\begin{array}{l}\text { Date of } \\
\text { Accident }\end{array}$ & $\begin{array}{c}\text { Accident } \\
\text { Description }\end{array}$ & $\begin{array}{l}\text { No. } \\
\text { of } \\
\text { Vhel }\end{array}$ & $\begin{array}{l}\text { Acc. } \\
\text { vel. } \\
\text { (mph) }\end{array}$ & $\begin{array}{c}\text { Fall } \\
\text { ht. } \\
(f t .)\end{array}$ & $\begin{array}{l}\text { Fire } \\
Y / N \\
\text { (dur) }\end{array}$ & $\begin{array}{c}\text { Object Struck } \\
\text { Description }\end{array}$ \\
\hline \multicolumn{9}{|l|}{ Kansas } \\
\hline Atchison & NMKC79FR024 & $1 / 17 / 79$ & HtoH Col. & $2 T$ & 60 & 0 & $Y(100 M)$ & Train \\
\hline Fort Scott & NMKC79FR036 & $3 / 11 / 79$ & Derail & 147 & 25 & 6 & N & RR Bed, RR Car \\
\hline Hecla & NMKC79FR001 & $10 / 5 / 78$ & Htos Col. & $2 \mathrm{~T}$ & 32 & 0 & $N$ & Train \\
\hline Lawrence & N/RAR-80-4 & $10 / 2 / 79$ & Derail & 20 & 80 & NS & $N$ & RR Bed, RR Car \\
\hline Lehigh & DOT B23-70 & $11 / 19 / 69$ & Derail & 36 & 27 & 0 & $Y(N S)$ & RR Bed, RR Car \\
\hline Malvern & N/RAR-75-1 & $7 / 5 / 74$ & Derail & 21 & 77 & NS & $N$ & RR Bed, RR Car \\
\hline \multicolumn{9}{|c|}{ Kansas/Missouri } \\
\hline $\begin{array}{c}\text { Fort Scott/ } \\
\text { Liberal } \\
\because\end{array}$ & NMKC 79FR020 & $1 / 3 / 79$ & Derail & 68 & 50 & 0 & $N$ & RR Bed, RR Car \\
\hline \multicolumn{9}{|l|}{ Kentucky } \\
\hline Fort Knox & N/RAR-83-07 & $3 / 22 / 83$ & Derail & 1 & 28 & NS & N & RR Bed, RR Car \\
\hline Hanson & NDCA79FR020 & $1 / 7 / 79$ & Derail & 115 & 42 & 0 & $N$ & RR Bed, RR Car \\
\hline Mularaugh & N/RAR-81-1 & $7 / 26 / 80$ & Derail & 1 & 35 & NS & $Y(5760 M)$ & RR Bed, RR Car \\
\hline Stepstone & NATL77FR007 & $11 / 8 / 76$ & Derail & 54 & 38 & 20 & $N$ & RR Bed, RR Car \\
\hline
\end{tabular}


Table A.7

Train Accidents

\begin{tabular}{|c|c|c|c|c|c|c|c|c|}
\hline Location. & $\begin{array}{l}\text { Report } \\
\text { Source }\end{array}$ & $\begin{array}{l}\text { Date of } \\
\text { Accident }\end{array}$ & $\begin{array}{l}\text { Accident } \\
\text { Description }\end{array}$ & $\begin{array}{c}\text { No. } \\
\text { of } \\
\text { Vhcl } 1\end{array}$ & $\begin{array}{l}\text { Acc. } \\
\text { vel. } \\
\text { (mph) }\end{array}$ & $\begin{array}{c}\text { Fall } \\
\text { ht. } \\
(\mathrm{ft.})\end{array}$ & $\begin{array}{l}\text { Fire } \\
\text { Y/N } \\
\text { (dur) }\end{array}$ & $\begin{array}{c}\text { Object Struck } \\
\text { Description }\end{array}$ \\
\hline \multicolumn{9}{|l|}{ Louisiana } \\
\hline Livingston & N/RAR-83-05 & $9 / 28 / 82$ & Derail & 1 & 40 & NS & $Y(8 D)$ & RR Bed, RR Car \\
\hline Meeler & N/RAR-75-9 & $5 / 30 / 75$ & $\mathrm{HtoH} \mathrm{Col}$. & $2 T$ & 48 & 0 & $N$ & Train \\
\hline Taft & N/RAR-73-6 & $2 / 21 / 73$ & $\mathrm{HtoH} \mathrm{Col}$. & $2 T$ & 43 & 0 & $Y(N S)$ & Train \\
\hline West Monroe & NFTW79FR008 & $10 / 24 / 78$ & Derai1 & 105 & 10 & 6 & $N$ & RR Bed, RR Car \\
\hline \multicolumn{9}{|l|}{ Maryland } \\
\hline Baltimore & N/RAR-78-1 & $6 / 12 / 77$ & $\mathrm{HtoH} \mathrm{Col}$. & $2 T$ & 30 & 0 & $Y(N S)$ & Train \\
\hline Corsey & FRA $\quad$ C-17-72 & NS & Derail & 55 & .55 & NS & NS & RR Bed, RR Car \\
\hline Germantown & N/RAR- $81-6$ & $2 / 9 / 81$ & $\mathrm{HtoH} \mathrm{Col}$. & 2 & 88 & NS & NS & Train \\
\hline Seabrook & N/RAR $-79-3$ & $6 / 9 / 78$ & HtoH Col. & $2 T$ & 35 & NS & $\mathrm{N}$ & Train \\
\hline & & & & & & & & . \\
\hline \multicolumn{9}{|l|}{ Massachusetts } \\
\hline Beverly & N/RAR-82-1 & $8 / 11 / 81$ & $\mathrm{HtoH} \mathrm{Col}$. & 2 & 19 & NS & $\mathrm{N}$ & Train \\
\hline Somerville & N/HZM-81-1 & $4 / 3 / 80$ & Htos Col. & 2 & 4 & NS & $N$ & Tank Car \\
\hline
\end{tabular}


Table A.7

Train Accidents

\begin{tabular}{|c|c|c|c|c|c|c|c|c|}
\hline Location & $\begin{array}{l}\text { Report } \\
\text { Source }\end{array}$ & $\begin{array}{l}\text { Date of } \\
\text { Accident }\end{array}$ & $\begin{array}{c}\text { Accident } \\
\text { Description }\end{array}$ & $\begin{array}{l}\text { No. } \\
\text { of } \\
\text { Vhcl }\end{array}$ & $\begin{array}{l}\text { Acc. } \\
\text { ve T. } \\
\text { (mph) }\end{array}$ & $\begin{array}{l}\text { Fall } \\
\text { ht. } \\
\text { (ft.) }\end{array}$ & $\begin{array}{l}\text { Fire } \\
\text { Y/N } \\
\text { (dur) }\end{array}$ & $\begin{array}{c}\text { 0bject Struck } \\
\text { Description }\end{array}$ \\
\hline Michigan & $\therefore \quad \therefore$ & & & & & & & \\
\hline Kopje & " & & & & & & & \\
\hline (Woodlnad) & NEHI78FR024? & NS & Derail & 38 & 34 & 8 & NS & RR Bed, RR Car \\
\hline $\begin{array}{l}\text { Lansing } \\
\qquad . . . i_{2}\end{array}$ & $\begin{array}{r}\text { NCHI7.9FRO15 } \\
41\end{array}$ & $\begin{array}{c}12 / 28 / 78 \\
\cdots\end{array}$ & Derail & 74 & 40 & 0 & $N$ & RR Bed, RR Car \\
\hline Minnesota & $\because \cdots+\cdots$ & $\therefore$ & & & & & & \\
\hline DeGraff & NMK'C 6FR126 & $7 / 4 / 76$ & Derail & 61 & NS & 0 & $Y(3 M)$ & RR Bed, RR Car \\
\hline Forbes & NMKC7.6FR059 & NS : & Derail & 119 & 30 & 30 & $Y(2 \mathrm{H})$ & RR Bed, RR Car \\
\hline Hills sat & NMKC7.9FRO12: & NS & Derail & 44 & NS & NS & $\mathrm{N}$ & RR Bed, RR Car \\
\hline $\begin{array}{r}\text { Nashau } \\
\text {, }\end{array}$ & NMKCT.9FRO11 & $.1 / 30 / 78$ & Derail & 55 & 40 & 9 & N & RR Bed, RR Car \\
\hline \multicolumn{9}{|l|}{ Mississippi } \\
\hline Goodman & N/RAR $-77-3$ & $6 / 30 / 76$ & Derail & 13 & 88 & 0 & $N$ & RR Bed, RR Car \\
\hline Laurel & N/RAR-69- & $1 / 25 / 69$ & Derail & 144 & 30 & 0 & $Y(60 \mathrm{H})$ & RR Bed, RR Car \\
\hline Missour, $\mathbf{j}_{i}$ & $\because \cdots x^{\prime}$ & & & & & & & \\
\hline Crystal City & N/RAR-84-01 & $7 / 18 / 83$ & Derail & 94 & 52 & 25 & $\mathrm{~N}$ & RR Bed, RR Car \\
\hline Dexter $\cdots$ & NMKC79FR003 & $10 / 10 / 78$ & HtoH Col. & $2 T$ & NS & 0 & N & Train \\
\hline Dresden & NMKC 79FR025 & $1 / 23 / 79$ & Derail & 38 & 50 & 8 & $N$ & RR Bed, RR Car \\
\hline
\end{tabular}


Table A.7

Train Accidents

\begin{tabular}{|c|c|c|c|c|c|c|c|c|}
\hline Location & $\begin{array}{l}\text { Report } \\
\text { Source }\end{array}$ & $\begin{array}{l}\text { Date of } \\
\text { Accident }\end{array}$ & $\begin{array}{l}\text { Accident } \\
\text { Description }\end{array}$ & $\begin{array}{l}\text { No. } \\
\text { of } \\
\text { Vhcl }\end{array}$ & $\begin{array}{l}\text { Acc. } \\
\text { vel. } \\
\text { (mph) }\end{array}$ & $\begin{array}{c}\text { Fall } \\
\text { ht. } \\
(\mathrm{ft.})\end{array}$ & $\begin{array}{l}\text { Fire } \\
\text { Y/N } \\
\text { (dur) }\end{array}$ & $\begin{array}{c}\text { Object Struck } \\
\text { Description }\end{array}$ \\
\hline \multicolumn{9}{|c|}{ Missouri (continued) } \\
\hline Kansas City & NMKC79FR015 & $12 / 16 / 78$ & Derail & 155 & 20 & 24 & $Y(20 M)$ & $\begin{array}{l}\text { RR Bridge, RR } \\
\text { Bed, RR Car }\end{array}$ \\
\hline Randles & NMKC79FR033 & $2 / 9 / 79$ & Drl. Col. & $2 \mathrm{~T}$ & 25 & 0 & $N$ & $\begin{array}{l}\text { RR Bed, RR Car, } \\
\text { Train }\end{array}$ \\
\hline Springfield & NMKC79FR022 & $1 / 10 / 79$ & Derail & 124 & 56 & 16 & $Y(N S)$ & RR Bed, RR Car \\
\hline \multicolumn{9}{|l|}{ Montana } \\
\hline Belt & N/RAR-77-7 & $11 / 26 / 76$ & Derai 1 & 126 & 38 & NS & $Y(12 H)$ & RR Bed, RR Car \\
\hline Browning & NSEA79FR003 & $10 / 23 / 78$ & Side Col. & $2 T$ & 25 & 30 & $\mathrm{~N}$ & Train \\
\hline Butte & NSEA79FR013 & $12 / 18 / 78$ & Derail & 81 & 26 & 0 & NS & RR Bed, RR Car \\
\hline Curry & FRA $C-7-72$ & NS & Derail & 84 & 50 & NS & NS & RR Bed, RR Car \\
\hline Essex & NSEA79FR001 & $10 / 3 / 78$ & Derail & 35 & 59 & 0 & $N$ & RR Bed, RR Car \\
\hline Glacier Park & N/RAR-80-6 & $3 / 14 / 80$ & Derail & 10 & 37 & 12 & $N$ & RR Bed, RR Car \\
\hline Greycliff & NSEA79FR006 & $11 / 3 / 78$ & Derail & 74 & 55 & 12 & $Y(N S)$ & RR Bed, RR Car \\
\hline Havre & NSEA79FR008 & $11 / 14 / 78$ & Derail & 81 & 60 & 18 & N & RR Bed, RR Car \\
\hline Lohman & N/RAR-79-7 & $3 / 28 / 79$ & Derail & 14 & 74 & 0 & $N$ & RR Bed, RR Car \\
\hline Zurich & NSEA79FR009 & $12 / 8 / 78$ & HtoH Col. & $2 T$ & 35 & 0 & $N$ & Train \\
\hline
\end{tabular}


Table A.7

Train Accidents

\begin{tabular}{|c|c|c|c|c|c|c|c|c|}
\hline Location & $\begin{array}{l}\text { Report } \\
\text { Source }\end{array}$ & $\begin{array}{l}\text { Date of } \\
\text { Accident }\end{array}$ & $\begin{array}{c}\text { Accident } \\
\text { Description }\end{array}$ & $\begin{array}{l}\text { No. } \\
\text { of } \\
\text { Vhcl }\end{array}$ & $\begin{array}{l}\text { Acc. } \\
\text { vel. } \\
\text { (mph) }\end{array}$ & $\begin{array}{c}\mathrm{Fall} \\
\text { ht. } \\
(\mathrm{ft.})\end{array}$ & $\begin{array}{l}\text { Fire } \\
Y / N \\
\text { (dur) }\end{array}$ & $\begin{array}{c}\text { Object Struck } \\
\text { Description }\end{array}$ \\
\hline \multicolumn{9}{|l|}{ Nebraska } \\
\hline Angora & N/RAR-80-7 & $2 / 16 / 80$ & $\mathrm{HtoH} \mathrm{Col}$. & $2 T$ & 49 & 0 & N & Train \\
\hline Arlington & NMKC79FR031 & $1 / 31 / 79$ & Derail & 82 & 40 & 0 & $\mathrm{~N}$ & RR Bed, RR Car \\
\hline Crete & N/RAR-71-2 & $2 / 18 / 69$ & Derail & 169 & 52 & 0 & N & RR Bed, RR Car \\
\hline Glenville & NS & $5 / 19 / 76$ & Derail & 70 & 68 & 0 & $N$ & RR Bed, RR Car \\
\hline Gothenburg & NMKC 79FR035 & $3 / 12 / 79$ & Derail & 109 & 60 & 0 & $N$ & RR Bed, RR Car \\
\hline Hastings & N/RAR-77-1 & $8 / 2 / 76$ & Derail & 119 & 45 & 0 & $N$ & RR Bed, RR Car \\
\hline Josselyn & NMKC7FR006 & NS & Derail & 116 & 70 & NS & NS & RR Bed, RR Car \\
\hline Marsland & NMKC79FR026 & $1 / 25 / 79$ & Derail & 110 & 45 & 40 & $N$ & RR Bed, RR Car \\
\hline Potter & NMKC77FR004 & $11 / 13 / 76$ & Derail & 90 & NS & 0 & $Y(1 M)$ & RR Bed, RR Car \\
\hline Ralston & N/RAR-77-8 & $12 / 16 / 76$ & Derail & 12 & 53 & 40 & $N$ & RR Bed, RR Car \\
\hline \multicolumn{9}{|l|}{ Nevada } \\
\hline Elburz & NOAK76FR127 & $7 / 4 / 76$ & Derail & 41 & NS & 10 & $\mathrm{~N}$ & RR Bed, RR Car \\
\hline Hoya & NOAK79FR015 & $12 / 4 / 78$ & HtoH Col. & $2 T$ & 22 & 0 & $Y(2.5 H)$ & RR Cars \\
\hline \multicolumn{9}{|l|}{ New Jersey } \\
\hline Edison & N/RAR-79-10 & $4 / 20 / 79$ & HtoH Col. & $2 T$ & NS & -0 & $Y(5 M)$ & Truck, Machinery \\
\hline Linden & N/RAR-80-12 & $7 / 9 / 80$ & Derail 1 & 2 & 30 & NS & $N$ & RR Bed, RR Car \\
\hline
\end{tabular}


Table A.7

Train Accidents

\begin{tabular}{|c|c|c|c|c|c|c|c|c|}
\hline Location & $\begin{array}{l}\text { Report } \\
\text { Source }\end{array}$ & $\begin{array}{l}\text { Date of } \\
\text { Accident }\end{array}$ & $\begin{array}{c}\text { Accident } \\
\text { Description }\end{array}$ & $\begin{array}{l}\text { No. } \\
\text { of } \\
\text { Vhc } 1\end{array}$ & $\begin{array}{l}\text { Acc. } \\
\text { vel. } \\
\text { (mph) }\end{array}$ & $\begin{array}{c}\text { Fall } \\
\text { ht. } \\
\text { (ft.) }\end{array}$ & $\begin{array}{l}\text { Fire } \\
Y / N \\
\text { (dur) }\end{array}$ & $\begin{array}{l}\text { Object Struck } \\
\text { Description }\end{array}$ \\
\hline
\end{tabular}

\section{New Mexico}

Des Moines

NDEN79FR001. 10/25/78 Derai1

62

23 NS N

RR Bridge, RR

Bed, RR. Car

\section{New York}

\begin{tabular}{|c|c|c|c|c|c|c|c|c|}
\hline Brooklyn & N/RAR-82-2 & $7 / 3 / 81$ & HtoH Col. & 2 & 12.7 & NS & $N$ & Subway Car \\
\hline Dobbs Ferry & N/RAR-81-4 & $11 / 7 / 80$ & HtoH Col. & 2 & 10 & NS & $Y(15 M)$ & Power Car \\
\hline New York City & N/RAR-75-8 & $1 / 2 / 75$ & $\mathrm{HtoH} \mathrm{Col}$. & 2 & 35 & NS & N & Rail Car \\
\hline NY C ity Subway & N/RAR-79-8 & $12 / 12 / 78$ & Derail & 8 & NS & 0 & $Y(N S)$ & $\begin{array}{l}\text { RR Bed, RR Car, } \\
\text { Concrete Wall }\end{array}$ \\
\hline NY City Subway & N/RAR-79-8 & $1 / 15 / 79$ & Derail & 10 & NS & 0 & N & RR Bed, RR Car \\
\hline NY City Subway & N/RAR-79-8 & $2 / 14 / 79$ & Derail & 10 & NS & NS & N & RR Bed \\
\hline NY City Subway & N/RAR-79-8 & $3 / 21 / 79$ & Derail & 8 & NS & 0 & $N$ & RR Bed \\
\hline Oneonta & N/RAR-74-4 & $2 / 12 / 74$ & Derail & 125 & 32 & 0 & $Y(7 D)$ & RR Bed, RR Car \\
\hline orth Carolina & & & & & & & & \\
\hline Laleview & N/RAR- $80-10$ & $4 / 2 / 80$ & HtoH $\mathrm{Col}$. & $2 T$ & 35 & 0 & $N$ & Train \\
\hline Spencer & N/RAR-78-3 & $10 / 8 / 77$ & Side Col. & $2 T$ & 50 & 0 & $N$ & $\begin{array}{l}\text { Train, RR Bed, RR } \\
\text { Car }\end{array}$ \\
\hline
\end{tabular}


Table A.7

Train Accidents

\begin{tabular}{llllll}
\hline Location & $\begin{array}{l}\text { Report } \\
\text { Source }\end{array}$ & $\begin{array}{l}\text { Date of } \\
\text { Accident }\end{array}$ & $\begin{array}{c}\text { Accident } \\
\text { Description }\end{array}$ & $\begin{array}{c}\text { No. } \\
\text { of } \\
\text { Vhcl vel. Fall }\end{array}$ & $\begin{array}{c}\text { Fire } \\
\text { (mph) }\end{array}$ \\
\hline
\end{tabular}

\section{Morth Dakota}

$\begin{array}{lllllllll}\text { Fairmont } & \text { NMKC79FR019 } & 12 / 31 / 78 & \text { Derail } & 83 & 40 & 0 & \text { N } & \text { RR Bed, RR Car } \\ \text { Walcott } & \text { NMKC79FR034 } & 2 / 17 / 79 & \text { Derail } & 64 & 48 & 15 & \text { N } & \text { RR Bed, RR Car } \\ \text { White Earth } & \text { NMKC79FR021. } & 1 / 7 / 79 & \text { Derail } & 77 & 45 & 0 & \text { N } & \text { RR Bed, RR Car }\end{array}$

\section{Ohio}

\begin{tabular}{|c|c|c|c|c|c|c|c|c|}
\hline Albany $:$ & $\begin{array}{c}\text { FRA }: C-68-7: 2 \\
\cdots\end{array}$ & NS & Derail & 93 & 30 & NS & $Y(N S)$ & $\begin{array}{l}\text { RR Bed, RR Car, } \\
\text { Creek Bed }\end{array}$ \\
\hline Circleville & $\begin{array}{l}\text { Columbus, } \mathrm{OH} \\
\text { News }\end{array}$ & $2 / 17 / 81$ & Derail & 490 & NS & 0 & N & RR Bed, RR Car \\
\hline Cleveland & N/RAR-75-3 & $5 / 8 / 74$ & Brdg Col. & 96 & 33 & 25 & $N$ & Drawbridge \\
\hline Columbus & ICC 4036 & NS & Derail & 29 & 43 & 0 & $Y(2 H)$ & RR Bed, RR Car \\
\hline Huntington & FRA $B-3-72$ & NS & Derail & 108 & 38 & NS & $Y(3 H)$ & RR Bed, RR Car \\
\hline Leetonia & N/RAR-76-2 & $6 / 6 / 75$ & $\mathrm{HtoH} \mathrm{Col}$. & $2 T$ & 29 & 0 & $N$ & Train \\
\hline Leetonia & NCHII9FR005 & $11 / 1 / 78$ & $\mathrm{HtoH} \mathrm{Col}$. & 5 & 32 & 0 & $N$ & Train \\
\hline Lodi & NCHI RR7.6081 & $5 / 30 / 76$ & Derail & 72 & 57 & 15 & $Y(2 H)$ & RR Bed, RR Car \\
\hline Pettisville & N/RAR-76-10 & $2 / 4 / 76$ & $\mathrm{HtOH} \mathrm{Col}$. & $2 \mathrm{~T}$ & 70 & 0 & $Y(N S)$ & Train \\
\hline Pemberville & NCHI79FR012 & $12 / 3 / 78$ & Derail & 185 & 35 & 0 & $N$ & RR Bed, RR Car \\
\hline St. Louisville & Uticá News & NS & Derail & $83+$ & 25 & 0 & $N$ & RR Bed, RR Car \\
\hline
\end{tabular}


Table A.7

Train Accidents

\begin{tabular}{lllllll}
\hline Location & $\begin{array}{l}\text { Report } \\
\text { Source }\end{array}$ & $\begin{array}{l}\text { Date of } \\
\text { Accident }\end{array}$ & $\begin{array}{c}\text { Accident } \\
\text { Description }\end{array}$ & $\begin{array}{c}\text { No. Acc. Fall Fire } \\
\text { of vel. } \\
\text { Vhcl }\end{array}$ & $\begin{array}{c}\text { Ft. } \\
\text { (mph) } \\
\text { (ft.) }\end{array}$ & $\begin{array}{c}\text { Object Struck } \\
\text { (dur) }\end{array}$ \\
\hline
\end{tabular}

Ohio (continued)

$\begin{array}{lllllllll}\text { Wooster } & \text { NCHI79FR008 } & 11 / 18 / 78 & \text { HtoS Col. } & 2 T & 23 & 0 & \text { N } & \text { Train, Tower } \\ \text { Wooster } & \text { NCHI77FR013 } & 12 / 23 / 76 & \text { Derail } & 131 & 30 & 15 & \text { Y(10M) RR Bed, RR Car }\end{array}$

\title{
Oklahoma
}

Alva

NFTW79FR028 3/21/79 Derail

Leonard

Mustang

ASME RAIL

TRANSPORT

PROCEEDINGS NS Derail

N/RAR-75-6 9/1/74 HtoH Col.

Sallisaw

NFTW79FR011

$11 / 6 / 78$

Derail

83

42

$5 \quad N$

RR Bed, RR Car

Oregon

Hunt ington

NSEA79FR012

12/18/78 Derai1

97

$23 \quad 35$ NS NS

$\begin{array}{rrrr}2 T & 40 & 0 & Y(N S)\end{array}$

RR Bed, RR Car

Train

RR Bed, RR Car

$\begin{array}{ll}52 & 37\end{array}$

60

\author{
RR Bed, RR Car
}


Table A.7

Train Accidents

\begin{tabular}{|c|c|c|c|c|c|c|c|c|}
\hline Location & $\begin{array}{l}\text { Report } \\
\text { Source }\end{array}$ & $\begin{array}{l}\text { Date of } \\
\text { Accident }\end{array}$ & $\begin{array}{l}\text { Accident } \\
\text { Description }\end{array}$ & $\begin{array}{c}\text { No. } \\
\text { of } \\
\text { Vhcl }\end{array}$ & $\begin{array}{l}\text { Acc. } \\
\text { vel. } \\
\text { (mph) }\end{array}$ & $\begin{array}{c}\text { Fall } \\
\text { ht. } \\
(\mathrm{ft.})\end{array}$ & $\begin{array}{l}\text { Fire } \\
Y / N \\
\text { (dur) }\end{array}$ & $\begin{array}{l}\text { Object Struck } \\
\text { Description }\end{array}$ \\
\hline \multicolumn{9}{|l|}{ Pennsylvania } \\
\hline Big Run & NNYC79FR031 & $2 / 13 / 79$ & Derail & 74 & 34 & 0 & $N$ & RR Bed, RR Car \\
\hline Bristol & N/RAR-82-5 & $3 / 29 / 82$ & HtoH Col. & $2 T$ & 22 & 0 & $N$ & Train \\
\hline Bryant & NNYC79FR021 & NS & Derail & 98 & 30 & 5 & $\mathrm{~N}$ & RR Bed, RR Car \\
\hline Culmerville & NNYC79FR003 & $10 / 10 / 78$ & Derail & 145 & 35 & 0 & $N$ & RR Bed, RR Car \\
\hline Herndon & N/RAR-73-3 & $3 / 12 / 72$ & HtoH Col. & $2 \mathrm{~T}$ & 60 & 0 & $Y(N S)$ & Train \\
\hline Munch. & N/RAR-79-6 & $1 / 31 / 79$ & $\mathrm{HtoH} \mathrm{Col}$. & $2 \mathrm{~T}$ & 30 & 0 & $N$ & Train \\
\hline North Wales & N/RAR-80-11 & $7 / 17 / 80$ & $\mathrm{HtoH} \mathrm{Col}$. & 2 & 39 & NS & $N$ & Electric Car \\
\hline Philadelphia & N/RAR-80-5 & $10 / 16 / 79$ & 2HTOT CL. & $3 T$ & 28 & 0 & $N$ & Trains \\
\hline Royers ford & N/RAR-80-2 & $10 / 1 / 79$ & $\mathrm{HtoH} \mathrm{Col}$. & $2 T$ & 45 & 0 & N & Train \\
\hline Weatherby & NNYC78FA015 & NS & Derail & 145 & NS & 30 & NS & RR Bed, RR Car \\
\hline \multicolumn{9}{|l|}{ South Carolina } \\
\hline Denmark & NATL79FR013 & $1 / 7 / 79$ & Derail & 103 & 40 & 0 & $N$ & RR Bed, RR Car \\
\hline Florence & N/RAR-78-6 & $2 / 24 / 78$ & Derai 1 & 20 & 20 & 0 & $Y(N S)$ & RR Bed, RR Car \\
\hline
\end{tabular}


Table A.7

Train Accidents

\begin{tabular}{|c|c|c|c|c|c|c|c|c|}
\hline Location & $\begin{array}{l}\text { Report } \\
\text { Source }\end{array}$ & $\begin{array}{l}\text { Date of } \\
\text { Accident }\end{array}$ & $\begin{array}{l}\text { Accident } \\
\text { Description }\end{array}$ & $\begin{array}{c}\text { No. } \\
\text { of } \\
\text { Vhcl }\end{array}$ & $\begin{array}{l}\text { Acc. } \\
\text { vel. } \\
\text { (mph) }\end{array}$ & $\begin{array}{c}\text { Fall } \\
\text { ht. } \\
(\mathrm{ft.})\end{array}$ & $\begin{array}{l}\text { Fire } \\
Y / N \\
\text { (dur) }\end{array}$ & $\begin{array}{l}\text { Object Struck } \\
\text { Description }\end{array}$ \\
\hline \multicolumn{9}{|l|}{ Tennessee } \\
\hline Brownsville & NATL77FR020 & $2 / 17 / 77$ & Derail & 101 & 49 & 20 & $Y(4 H)$ & RR Bed, RR Car \\
\hline Fosterville & FRA $C-5-72$ & NS & Derail & 123 & 47 & NS & NS & RR Bed, RR Car \\
\hline N Johnsonville & N/RAR-82-4 & $12 / 28 / 81$ & $\mathrm{HtoH} \mathrm{Col}$. & 2 & 25 & 45 & $\mathrm{~N}$ & Caboose \\
\hline Pulaski & R/RAR-76-6 & $10 / 1 / 75$ & Derail & 14 & 65 & 40 & $\mathrm{~N}$ & RR Bed, RR Car \\
\hline Roddy & NATL79FR012 & $12 / 24 / 78$ & Derail & 231 & 44 & 6 & $N$ & RR Bed, RR Car \\
\hline Waverly & N/RAR-79-1 & $2 / 22 / 78$ & Derail & 120 & 35 & 0 & $Y(6 H)$ & RR Bed, RR Car \\
\hline
\end{tabular}

辳 Texas

$\begin{array}{llllrrrrl}\text { Britton } & \text { NFTW79FR016 } & 12 / 10 / 78 & \text { Derail } & 98 & 25 & 7 & \text { N } & \text { RR Bed, RR Car } \\ \text { Cotulla } & \text { N/RAR-74-3 } & 12 / 1 / 73 & \text { HtoH Col. } & 2 T & 40 & 0 & Y(1.5 H) & \begin{array}{l}\text { Train } \\ \text { Dallas }\end{array} \\ & \text { San Jose News } & 2 / 21 / 81 & \text { Derail } & 60 & \text { NS } & 50 & Y(4 H) & \begin{array}{l}\text { RR Bed, RR Car, } \\ \text { Bridge }\end{array} \\ \text { Garland } & \text { NFTW77FR007 } & 3 / 20 / 77 & \text { Derail } & 44 & \text { NS } & 0 & Y(N S) & \text { RR Bed, RR Car } \\ \text { Houston } & \text { N/RAR-75-7 } & 9 / 21 / 74 & \text { Yard Col. } & 503 & 20 & 0 & Y(9 H) & \text { RR C.ars } \\ \text { Houston } & \text { N/RAR-72-6 } & 10 / 19 / 71 & \text { Derail } & 88 & 45 & 45 & Y(5 H) & \text { RR Bed, RR Car } \\ \text { Marquez } & \text { NFTW79FR005 } & 10 / 13 / 78 & \text { Derail } & 94 & 30 & 0 & \text { N } & \begin{array}{l}\text { RR Bed, RR Car, } \\ \text { Paxton }\end{array} \\ & \text { N/HZM-80-1 } & 9 / 8 / 79 & \text { Derail } & 56 & 30 & 15 & Y(N S) & \text { RR Bed, RR Car }\end{array}$


Table A.7

Train Accidents

\begin{tabular}{|c|c|c|c|c|c|c|c|c|}
\hline Location & $\begin{array}{l}\text { Report } \\
\text { Source }\end{array}$ & $\begin{array}{l}\text { Date of } \\
\text { Accident }\end{array}$ & $\begin{array}{l}\text { Accident } \\
\text { Description }\end{array}$ & $\begin{array}{l}\text { No. } \\
\text { of } \\
\text { Vhcl }\end{array}$ & $\begin{array}{l}\text { Acc. } \\
\text { vel. } \\
\text { (mph) }\end{array}$ & $\begin{array}{c}\mathrm{Fall} \\
\text { ht. } \\
(\mathrm{ft} .)\end{array}$ & $\begin{array}{l}\text { Fire } \\
Y / N \\
\text { (dur) }\end{array}$ & $\begin{array}{l}\text { Object Struck } \\
\text { Description }\end{array}$ \\
\hline \multicolumn{9}{|c|}{ Texas (continued) } \\
\hline Temple & N/RAR-83-08 & $3 / 17 / 83$ & $\mathrm{HtoH} \mathrm{Col}$. & 8 & 35 & NS & $N$ & Freight Car \\
\hline Tyler & $\begin{array}{l}\text { NFTW79FR007 } \\
\end{array}$ & $10 / 22 / 78$ & Derail & 79 & 45 & 12 & N & RR Bed, RR Car \\
\hline Utah & $\ldots \quad \therefore \quad \therefore \quad \cdots$ & & : & & & & & \\
\hline $\begin{array}{c}\text { Lakes ide } \\
\vdots\end{array}$ & NDEN76FR111 & $6 / 25 / 76$ & Derait & 52 & NS & 10 & N & $\begin{array}{l}\text { RR Bed, RR Car, } \\
\text { Lake }\end{array}$ \\
\hline
\end{tabular}

$\begin{array}{llll}\text { Virginia } & & \\ \text { Arlington } & \text { N/RAR-73-2 } & 4 / 27 / 72 & \text { HtoH Col. } \\ \text { Colonial Hghts N/RAR-83-04 } & 5 / 5 / 82 & \text { Derail } \\ \text { Crewe } & \text { N/RAR-82-3: } & 11 / 28 / 81 & \text { HtoS Col. } \\ \text { Elma : : } & \text { N/RAR-79-4: } & 12 / 3 / 78 & \text { Derail } \\ \text { Franconia } & \text { N/RAR-71-1 } & 1 / 27 / 70 & \text { Derail } \\ \text { Jarratt } & \text { N/RAR-76-11 } & 5 / 5 / 76 & \text { Derail } \\ \text { Rockfish } & \text { N/RAR-83-10 } & 4 / 3 / 83 & \text { Derail }\end{array}$


Table A.7

Train Accidents

\begin{tabular}{|c|c|c|c|c|c|c|c|c|}
\hline Location & $\begin{array}{l}\text { Report } \\
\text { Source }\end{array}$ & $\begin{array}{l}\text { Date of } \\
\text { Accident }\end{array}$ & $\begin{array}{l}\text { Accident } \\
\text { Description }\end{array}$ & $\begin{array}{l}\text { No. } \\
\text { of } \\
\text { Vhcl }\end{array}$ & $\begin{array}{l}\text { Acc. } \\
\text { vel. } \\
\text { (mph) }\end{array}$ & $\begin{array}{c}\text { Fall } \\
\text { ht. } \\
(\mathrm{ft.})\end{array}$ & $\begin{array}{l}\text { Fire } \\
Y / N \\
\text { (dur) }\end{array}$ & $\begin{array}{c}\text { Object Struck } \\
\text { Description }\end{array}$ \\
\hline \multicolumn{9}{|l|}{ Washington } \\
\hline Deer Park & NSEA79FR002 & $10 / 4 / 78$ & Derail & 41 & 23 & 0 & $N$ & RR Bed, RR Car \\
\hline \multicolumn{9}{|l|}{ Ephrata } \\
\hline (Naylor) & NSEA79FR021 & $2 / 28 / 79$ & Derail & 65 & 50 & NS & $\mathrm{N}$ & RR Bed, RR Car \\
\hline Kalama & NSEA76FR028 & $9 / 7 / 76$ & Derail & NS & 52 & 35 & $N$ & $\begin{array}{l}\text { RR Bed, RR Car, } \\
\text { River }\end{array}$ \\
\hline Kapows in & NSEA79FR023 & $3 / 6 / 79$ & Brdg Fail & 45 & 10 & 15 & $N$ & River, Bridge \\
\hline Tacoma & NSEA79FR025 & $3 / 22 / 79$ & Derail & 122 & 23 & 0 & $\mathrm{~N}$ & $\begin{array}{l}\text { RR Bed, RR Car, } \\
\text { RR Bridge }\end{array}$ \\
\hline Tukailla & NS & $10 / 8 / 77$ & $\mathrm{HtoH} \mathrm{Col}$. & $2 T$ & 50 & NS & $Y(N S)$ & Train \\
\hline Wenatchee & N/RAR-76-1 & $8 / 6 / 74$ & Explosion & 201 & 10 & 0 & $Y(N S)$ & NS \\
\hline \multicolumn{9}{|l|}{ West Virginia } \\
\hline Orleans Road & N/RAR-80-9 & $2 / 12 / 80$ & $\mathrm{HtoH} \mathrm{Col}$. & $2 \mathrm{~T}$ & 38 & 0 & $N$ & Train \\
\hline South Ruffner & NDCA79FR028 & $2 / 4 / 79$ & Side Col. & $2 T$ & 78 & 5 & $N$ & Train \\
\hline Welch & N/RAR-81-2 & $9 / 6 / 80$ & HtoS Col. & 2 & 38 & NS & NS & Freight Car \\
\hline
\end{tabular}


Table A.7

Train Accidents

\begin{tabular}{|c|c|c|c|c|c|c|c|c|}
\hline Location & $\begin{array}{l}\text { Report } \\
\text { Source }\end{array}$ & $\begin{array}{l}\text { Date of } \\
\text { Accident }\end{array}$ & $\begin{array}{l}\text { Accident } \\
\text { Description }\end{array}$ & $\begin{array}{c}\text { No. } \\
\text { of } \\
\text { Vhcl }\end{array}$ & $\begin{array}{l}\text { Acc. } \\
\text { vel. } \\
\text { (mph) }\end{array}$ & $\begin{array}{c}\text { Fall } \\
\text { ht. } \\
(\mathrm{ft.})\end{array}$ & $\begin{array}{l}\text { Fire } \\
Y / N \\
\text { (dur) }\end{array}$ & $\begin{array}{c}\text { Object Struck } \\
\text { Description }\end{array}$ \\
\hline \multicolumn{9}{|l|}{ Wisconsin } \\
\hline Columbus & NCHI79FR009 & $11 / 24 / 78$ & Derai1 & 70 & 50 & NS & $N$ & RR Bed, RR Car \\
\hline Cylon & FRA $C-15-72$ & NS & Derail & 95 & 45 & NS & NS & RR Bed, RR Car \\
\hline Franksville & NCHI79FR028 & $3 / 15 / 79$ & Derai1 & 81 & 40 & 0 & $\mathrm{~N}$ & RR Bed, RR Car \\
\hline Milawukee & NCHI79FR017 & $1 / 7 / 79$ & Derail & 55 & 38 & 0 & $N$ & RR Bed, RR Car \\
\hline Sturtevant & NCHI 79FR024 & $2 / 12 / 79$ & Derail & 84 & 40 & NS & $\mathrm{N}$ & RR Bed, RR Car \\
\hline \multicolumn{9}{|l|}{ Wyoming } \\
\hline Dale Junction & NDEN79FR007 & $1 / 22 / 79$ & Derail & 121 & 40 & 40 & $Y(56 \mathrm{H})$ & RR Bed, RR Car \\
\hline Granite & N/RAR-79-12 & $7 / 31 / 79$ & Derail & 85 & 75 & 0 & $\mathrm{~N}$ & RR Bed, RR Car \\
\hline Hermosa & N/RAR-81-3 & $10 / 16 / 80$ & $\mathrm{HtoH} \mathrm{Col}$. & 2 & 40 & NS & $N$ & raboose \\
\hline Leroy & NDEN79FR002 & $11 / 3 / 78$ & Derail & 92 & 60 & 0 & $N$ & RR Bed, RR Car \\
\hline Ramsey & N/RAR-79-9 & $3 / 29 / 79$ & $\mathrm{HtoH} \mathrm{Col}$. & $2 T$ & 48 & 0 & $N$ & Train \\
\hline Red Desert & NDEN77FR00 1 & NS & Derail & $66+$ & NS & NS & NS & RR Bed, RR Car \\
\hline Sheridan & N/RAR-72-4 & $3 / 28 / 71$ & Yard COL. & 14 & 15 & 0 & $\mathrm{~N}$ & RR Cars \\
\hline Wamsutter & $\begin{array}{c}\text { NDEN77FR007 } \\
.\end{array}$ & $2 / 23 / 77$ & $\begin{array}{l}\text { Derail } \\
\text { Side Col. }\end{array}$ & $N S-T$ & $67-54$ & $0-0$ & $N$ & $\begin{array}{l}\text { RR Bed, RR Car, } \\
\text { Train }\end{array}$ \\
\hline
\end{tabular}




\title{
APPENDIX B
}

\author{
List of Tables
}

Page

B.1 Petroleum Industry Accident Data Summary, 1973-1981 .............. B-3

B.2 Distribution of Velocities for Truck/Semitrailers Involved in Fatal and Injury Accidents in California, 1958-1967 ............ B-5

B.3 Distribution of Estimated Original Vehicle Velocities for A11 Types of Accidents, North Carolina, 1979-1981 ................ B-7

B.4 Distribution of Estimated Vehicle Impact Velocities for A11 Types of Accidents, North Carolina, 1979-1981 .............. B-8

B.5 Distribution of Train Velocities at Rail-Highway Grade-Crossing Accident/Incidents Involving Motor Vehicles, 1975-1982 ...................................... B-10

B.6 Summary of Objects Struck and Type of Accident for Accidents Involving U.S. Private and For-Hire Motor Carriers, $1973-1983$...................................... B-11

B.7 0bjects Struck During California Accidents, $1975-1983$............. B-12

B.8 0bjects Struck During California Accidents, Reordered According to Type of Accident, $1975-1983 \ldots \ldots \ldots \ldots \ldots \ldots \ldots \ldots$. B-15 


\section{APPENDIX B}

\section{Truck Accident Data}

\section{B.1 Introduction}

This appendix summarizes both the highway accident data which form the basis for the distribution of accident scenarios and the estimates of the probability distributions used in the probabilistic analysis of future truck accidents involving the transport of spent nuclear fuel. The primary sources of data are the Bureau of Motor Carrier Safety (BMCS), American Petroleum Institute (API), California Highway Patrol (CHP), and the California Department of Transportation (CALTRANS) reports on highway accidents. In addition, a Sandia report on severe accidents was the source of fire duration distributions and estimates of the probability of a fire.

Section B.2 discusses the data used to estimate the truck accident rate. Section B.3 discusses the distributions of truck velocities. Section B. 4 covers the distribution of train velocities used to analyze rail-highway grade crossing accidents. Section B.5 discusses the distribution of objects struck, and, finally, Sections B.6 and B.7 cover the fire accident data.

\section{B.2 Truck Accident Rate}

Information concerning truck accidents involving motor carriers of property that operate in interstate commerce is available in reports published by the BMCS of the U.S. Department of Transportation (DOT). B.1-B.13 Truck accidents are defined by the BMCS as occurrences involving a motor vehicle operated by a motor carrier subject to the Federal Motor Carrier Safety Regulations (49 CFR 390-397) resulting in (1) the death of one or more human beings; (2) bodily injury to one or more persons who, as a result, receives medical treatment away from the scene of the accident; and/or (3) total damage to all property aggregating dollar damage at or above the dollar damage threshold limit based on actual cost or reliable estimates.

Prior to 1973, the BMCS tabulated only those truck accidents with damage of $\$ 250$ or greater involving for-hire carriers, i.e., trucking firms that haul freight owned by another party. Since 1973, the BMCS has also tabulated 
accidents involving private, i.e., firms using their own, or leased, vehicles as part of their commercial operation to transport their own goods, as well as accidents of for-hire carriers. However, since 1973, the total vehicle miles have not been included in the BMCS reports. The accident rate for the period 1960-1972, $2.48 \times 10^{-6}$ accidents/vehicle-mile, is an estimate; however, (1) it is based on the experience some years ago, and (2) it is not clear what is defined as a truck. This definition is important because pickup trucks and vans, i.e., non tractor/semitrailer trucks, tend to have an accident rate closer to that of automobiles. Therefore, it was decided not to base the accident rate for this study on the BMCS data.

Another source of truck accident data is the database maintained by the API consisting of information supplied by petroleum industry companies. Accident data is available for the API for the period 1968 through 1981 for large trucks. B.14-B.18 Although a precise definition of an accident is not included in the reports, an accident rate based on the API data was used in this study. The API accident rate data was judged to be more reliable because shipments involving hazardous materials are usually more tightly controlled than shipments involving non-hazardous materials. In addition, the API data was judged to be most applicable to spent fuel shipment because trucks that transport gasoline type products are of similar size and weight to trucks that transport spent fuel. The API data is expected to be conservative because the average trip length of a gasoline truck is less than 28 miles and involves all types of roads. This will result in a higher accident rate than an accident rate based on cross-country trips that involve primarily interstates.

To allow for the imposition of the national speed 1 imit in 1973, only the data from 1973 through 1981 was used to estimate a truck accident rate. Table B.1 summarizes the API accident data for the years 1973 to 1981 . The estimated accident rate, $5.94 \mathrm{E} \times 10^{-6}$ accidents/truck-mile, is higher than the rate based on the BMCS data. 
Table B.1

Petroleum Industry Accident Data Summary, 1973-1981a/

\begin{tabular}{lccccc}
\hline Year & $\begin{array}{c}\text { No. of } \\
\text { Compy. }\end{array}$ & $\begin{array}{c}\text { No. of } \\
\text { Trucks }\end{array}$ & $\begin{array}{c}\text { No. of } \\
\text { Accidents }\end{array}$ & $\begin{array}{c}\text { Truck } \\
\text { Miles } \times 1000\end{array}$ & $\begin{array}{c}\text { Accident Rate/ } \\
\text { Truck-Mi } 7 \text { e }\end{array}$ \\
\hline 1973 & 73 & 20,046 & 3,804 & 508,783 & $7.4810^{-6}$ \\
1974 & 73 & 20,147 & 3,151 & 469,804 & $6.7110^{-6}$ \\
1975 & 69 & 29,071 & 4,089 & 779,260 & $5.2510^{-6}$ \\
1976 & 70 & 22,748 & 3,528 & 585,609 & $6.0210^{-6}$ \\
1977 & 69 & 21,508 & 2,784 & 519,446 & $5.3610^{-6}$ \\
1978 & 68 & 19,113 & 2,562 & 404,748 & $6.3310^{-6}$ \\
1979 & 63 & 21,414 & 2,889 & 467,939 & $6.1710^{-6}$ \\
1980 & 62 & 21,970 & 2,391 & 455,324 & $5.2510^{-6}$ \\
1981 & 81 & 21,158 & 2,445 & 465,571 & $5.2510^{-6}$ \\
\hline Total & & 197,175 & 27,643 & $4,656,484$ & $5.9410^{-6}$ \\
Avg/year & & 21,908 & 3,071 & 517,387 & \\
\hline
\end{tabular}

a/ American Petroleum Institute. B.14-B.18 


\section{B.3 Distributions of Velocity for Truck Accidents}

The velocity of the truck at the time of an accident is an important parameter in determining impact forces on cargos involved in highway accidents. This parameter, in combination with the angle of impact, is an estimate of the impact velocity of the cask at the time of the accident. The impact velocity, in combination with the cask orientation and the object struck or subsequent interaction of the truck with its environment after the accident begins, determines the forces and damage experienced by the cask. Thus, the distribution of truck velocities at the time of an accident is one of the necessary inputs into the probabilistic analys is of accidents involving spent fuel casks.

Considerable effort went into attempting to accumulate a database of accident data from past events which reasonably reflects what might be experienced by trucks transporting spent fuel casks in the future. To this end, annual reports on motor vehicle accidents, as accumulated by the CHP formed the basis for developing an appropriate collection of accident statistics. B.19-B.29 Although data from several classifications of accidents have been reported, e.g., all injury accidents, injury truck accidents, and all fatal accidents, we chose to estimate the desired distribution of velocities on fatal and injury accidents involving truck/semitrailers.

The distribution of velocities covering the years 1958-1967 is given in Table B.2. An important question with regard to the use of the data in Table B.2 as a basis for estimating velocities for future truck accidents is whether the traffic conditions in the 1958-1967 time period is comparable to traffic conditions which can be expected to be experienced in the future. Prior to 1959 California highway speed limits were $55 \mathrm{mph}$ for automobiles and $45 \mathrm{mph}$ for trucks (defined as trucks with three or more axles and any truck or truck tractor pulling one or more trailers) and cars with trailers. In 1959 the motor vehicle code was changed to 1 imit cars to $65 \mathrm{mph}$; however, trucks and cars with trailers were still limited to $45 \mathrm{mph}$ except on highways with four or more lanes (at least two lanes in each direction), where the speed limit was $50 \mathrm{mph}$. In 1963, the motor vehicle code was changed to limit cars on 
Table B.2

Distribution of Velocities for Truck/Semitrailers Involved in Fatal and Injury Accidents in California, 1958-1967a/

\begin{tabular}{cccc}
$\begin{array}{c}\text { Velocity } \\
\text { (mph) }\end{array}$ & $\begin{array}{c}\text { Number of } \\
\text { Accidents }\end{array}$ & $\begin{array}{c}\text { Fractional } \\
\text { Percent } \\
(\%)\end{array}$ & $\begin{array}{c}\text { Cumulative } \\
\text { Percent } \\
(\%)\end{array}$ \\
\hline 0 & 1,774 & 6.41 & 6.41 \\
$1-10$ & 4,143 & 14.96 & 21.37 \\
$11-20$ & 4,122 & 14.89 & 36.25 \\
$21-30$ & 4,248 & 15.34 & 51.59 \\
$31-40$ & 4,733 & 17.09 & 68.69 \\
$41-50$ & 7,264 & 26.23 & 94.92 \\
$51-60$ & 1,173 & 4.24 & 99.15 \\
$61-70$ & 171 & 0.62 & 99.77 \\
$>70$ & 63 & 0.23 & 100.00 \\
Tota 1 & 27,691 & 100.00 & \\
\hline al California Highway Patrol. B.19-B.29 & &
\end{tabular}


freeways to $70 \mathrm{mph}$ while trucks and cars with trailers were restricted to 50 mph on all highways.

The speed limits were again changed in 1967 to allow trucks and cars with trailers to travel up to $55 \mathrm{mph}$ over all highways. These regulations remained in effect until superseded by the national speed limit in 1973. Because the speed limits during the 1958-1967 time period were lower than the present 55 mph limit for all vehicles, the velocities in Table B.2 may be biased towards lower velocities. However, by choosing fatal and injury accidents, rather than al1 accidents (including non injury accidents), this bias has been somewhat compensated for because injury and fatal accidents generally involve higher velocities.

Accident data from North Carolina ${ }^{B} .30$ was used to estimate the effects of braking on impact velocity. Tables B. 3 and B.4 summarize the distribution of velocities for accidents involving all types of vehicles resulting in fatalities, injuries, or property damage for the years 1979-1981. In Table B.3, the velocities are based on estimates of the original vehicle velocity while in Table B.4 the velocities are estimates of the velocity at impact. As discussed in Section 5.0, a comparison of these two distributions was used as a basis for adjusting the distribution of truck velocities for the effects of braking during the evolution of an accident prior to vehicle impact.

\section{B.4 Distribution of Train Speeds at Rail-Highway Grade-Crossing Accidents}

The U.S. DOT Federal Railroad Administration (FRA) defines rail-highway grade-crossing accidents as any impact between railroad on-track equipment and an automobile, 'bus, truck, motorcycle, bicycle, farm vehicle, or pedestrian at a highway-rail grade crossing in which the amount of damage done to railroad equipment is at least a specified damage threshold limit. If the impact causes damage to railroad equipment less than the dollar damage threshold limit, it is classified as an incident. Prior to 1975, the damage threshold limit was $\$ 750$ and only rail-highway grade-crossing accidents were tabulated by the FRA. B.34 In 1975, the threshold was increased to $\$ 1750$ to account for 
Table B.3

Distribution of Estimated Original Vehicle Velocities for A11

Types of Accidents, North Carolina, 1979-1981a/

\begin{tabular}{|c|c|c|c|c|c|c|c|}
\hline $\begin{array}{c}\text { Velocity } \\
\text { (mph) }\end{array}$ & $\overline{1979}$ & $\frac{\text { Year }}{1980}$ & $\overline{1981}$ & Total & Avg. & $\begin{array}{c}\text { Fra. } \\
\text { Pct. } \\
(\%)\end{array}$ & $\begin{array}{c}\text { Cum. } \\
\text { Pct. } \\
(\%)\end{array}$ \\
\hline $\begin{array}{r}0 \\
1-5 \\
6-10 \\
11-15 \\
16-20 \\
21-25 \\
26-30 \\
31-35 \\
36-40 \\
41-45 \\
46-50 \\
51-55 \\
56-60 \\
61-65 \\
66-70 \\
71-75 \\
76-80 \\
81-85 \\
>85\end{array}$ & $\begin{array}{r}512 \\
22,191 \\
20,335 \\
13,846 \\
20,417 \\
17,336 \\
23,336 \\
33,147 \\
17,245 \\
22,028 \\
16,144 \\
15,336 \\
3,559 \\
2,071 \\
1,621 \\
751 \\
603 \\
134 \\
1243\end{array}$ & $\begin{array}{r}214 \\
19,976 \\
18,655 \\
12,697 \\
18,965 \\
16,388 \\
21,472 \\
33,147 \\
16,317 \\
21,049 \\
14,889 \\
14,301 \\
3,492 \\
1,907 \\
1,604 \\
685 \\
584 \\
127 \\
855\end{array}$ & $\begin{array}{r}188 \\
19,205 \\
17,865 \\
12,051 \\
18,042 \\
16,100 \\
21,582 \\
34,030 \\
16,075 \\
21,156 \\
14,315 \\
14,784 \\
3,261 \\
1,991 \\
1,476 \\
719 \\
539 \\
143 \\
807\end{array}$ & $\begin{array}{r}914 \\
61,372 \\
56,855 \\
38,594 \\
57,424 \\
49,824 \\
66,390 \\
100,324 \\
49,637 \\
64,233 \\
45,348 \\
44,421 \\
10,312 \\
5,969 \\
4,701 \\
2,155 \\
1,726 \\
404 \\
2,905\end{array}$ & $\begin{array}{r}305 \\
20,457 \\
18,952 \\
12,865 \\
19,141 \\
16,608 \\
22,130 \\
33,441 \\
16,546 \\
21,411 \\
15,116 \\
14,807 \\
3,437 \\
1,990 \\
1,567 \\
718 \\
575 \\
135 \\
968\end{array}$ & $\begin{array}{r}0.14 \\
9.25 \\
8.57 \\
5.82 \\
8.65 \\
7.51 \\
10.01 \\
15.12 \\
7.48 \\
9.68 \\
6.83 \\
6.69 \\
1.55 \\
0.90 \\
0.71 \\
0.32 \\
0.26 \\
0.06 \\
0.44\end{array}$ & $\begin{array}{r}0.14 \\
9.39 \\
17.96 \\
23.77 \\
32.43 \\
39.94 \\
49.94 \\
65.06 \\
72.54 \\
82.22 \\
89.06 \\
95.75 \\
97.31 \\
98.21 \\
98.92 \\
99.24 \\
99.50 \\
99.56 \\
100.00\end{array}$ \\
\hline Not Statedㅁ' & 45,590 & 43,290 & 42,526 & 131,406 & 43,802 & N/A & $N / A$ \\
\hline
\end{tabular}

a/. University of North Carolina Highway Safety Research Center. B. 30

b/ Excluded from percentage calculations. 
Table B. 4

Distribution of Estimated Vehicle Impact Velocities for A11 Types of Accidents, North Carolina, 1979-1981aㅢ

\begin{tabular}{|c|c|c|c|c|c|c|c|}
\hline $\begin{array}{c}\text { Velocity } \\
\text { (mph) }\end{array}$ & 1979 & $\frac{\text { Year }}{1980}$ & 1981 & Total & Avg. & $\begin{array}{c}\text { Fra. } \\
\text { Pct. } \\
(\%)\end{array}$ & $\begin{array}{c}\text { Cum. } \\
\text { Pct. } \\
(\%)\end{array}$ \\
\hline $\begin{array}{r}0 \\
1-5 \\
6-10 \\
11-15 \\
16-20 \\
21-25 \\
26-30 \\
31-35 \\
36-40 \\
41-45 \\
46-50 \\
51-55 \\
56-60 \\
61-65 \\
66-70 \\
71-75 \\
76-80 \\
81-85 \\
>85\end{array}$ & $\begin{array}{r}818 \\
30,831 \\
29,236 \\
20,279 \\
26,955 \\
18,904 \\
23,914 \\
19,368 \\
15,991 \\
11,589 \\
9,754 \\
4,936 \\
2,056 \\
818 \\
697 \\
250 \\
262 \\
58 \\
94\end{array}$ & $\begin{array}{r}413 \\
29,125 \\
28,273 \\
19,905 \\
26,958 \\
18,386 \\
23,301 \\
19,123 \\
15,091 \\
10,866 \\
9,249 \\
4,945 \\
2,028 \\
678 \\
687 \\
241 \\
251 \\
55 \\
87\end{array}$ & $\begin{array}{r}412 \\
29,181 \\
28,026 \\
19,811 \\
26,423 \\
18,619 \\
23,023 \\
18,706 \\
14,589 \\
10,554 \\
8,726 \\
4,730 \\
1,861 \\
691 \\
673 \\
239 \\
205 \\
52 \\
73\end{array}$ & $\begin{array}{r}1643 \\
89,137 \\
85,535 \\
59,995 \\
80,336 \\
55,909 \\
70,238 \\
57,197 \\
45,671 \\
33,009 \\
27,729 \\
14,611 \\
5,945 \\
2,187 \\
2,057 \\
730 \\
718 \\
165 \\
254\end{array}$ & $\begin{array}{r}548 \\
29,712 \\
28,512 \\
19,998 \\
26,779 \\
18,636 \\
23,413 \\
19,066 \\
15,224 \\
11,003 \\
9,243 \\
4,870 \\
1,982 \\
729 \\
686 \\
243 \\
239 \\
55 \\
85\end{array}$ & $\begin{array}{r}0.26 \\
14.08 \\
13.51 \\
9.48 \\
12.69 \\
8.83 \\
11.09 \\
9.03 \\
7.21 \\
5.21 \\
4.38 \\
2.31 \\
0.94 \\
0.35 \\
0.32 \\
0.12 \\
0.11 \\
0.03 \\
0.04\end{array}$ & $\begin{array}{r}0.26 \\
14.34 \\
27.85 \\
37.33 \\
50.02 \\
58.85 \\
69.94 \\
78.98 \\
86.19 \\
91.41 \\
95.79 \\
98.10 \\
99.03 \\
99.38 \\
99.71 \\
99.82 \\
99.93 \\
99.96 \\
100.00\end{array}$ \\
\hline Not Stated $b /$ & 60,635 & 50,952 & 50,261 & 161,848 & 53,949 & $N / A$ & $N / A$ \\
\hline
\end{tabular}

a/ University of North Carolina Highway Safety Research Center. B.30

b/ Excluded from percentage calculations. 
the effects of inflation. Also, at this time, the FRA started to include rail-highway grade-crossing incidents in their grade crossing accident data. B.31-B.38 This resulted in a substantial increase in the reported number of impacts between trains and other mobile objects in the grade-crossing accident data after 1975. Because of the difference in types of events recorded, only the rail-highway grade-crossing accident data after 1974 was used.

Table B.5 presents the distribution of train velocities at grade-crossing accidents/incidents involving motor vehicles. The reliability of the train accident/incident velocity at rail-highway grade-crossings can be considered good because railroad locomotives are equipped with accident recorders to record the train's velocity prior to, during, and after the accident, although on a very crude scale. The recorded train velocity while probably no more accurate than 5 to $10 \mathrm{mph}$, is certainly more reliable than after-the-fact velocity estimates made by investigating officers at highway accident sites.

\section{B.5 Highway Accident Object Frequency}

Data were collected from several sources to estimate the frequency of impact with particular objects. Two of the primary data sources were the CALTRANS for all vehicles and the BMCS for trucks.

Table B.6 presents the truck highway accident data obtained from the BMCS for the years 1973 through 1983.B.4-B.13 The object struck (for collision accidents) or accident type (for noncollision accidents) are categories as given by the BMCS. These categories are divided into nonfixed-object collisions, fixed-object collisionsys(for collision accidents), ran-off-road accidents, impact-with-roadbed accidents, or other noncollision accidents (for noncollision accidents). The BMCS data were divided this way in order to provide subcategories that would correspond with those defined by the CALTRANS in their reports on objects struck during highway accidents.

Table B.7 presents the primary objects struck during highway accidents, as reported by the CALTRANS for all vehicles for 1975 through 1983. B.39-B.47 A11 object struck subcategories are as defined by the CALTRANS and the object numbering system follows the CALTRANS convention. 
Table B.5

Distribution of Train Velocities at Rail-Highway Grade-Crossing Accident/Incidents Involving Motor Vehicles, 1975-1982 al

\begin{tabular}{|c|c|c|c|c|c|c|c|c|c|c|c|}
\hline $\begin{array}{l}\text { Velocity } \\
\text { (mph) }\end{array}$ & 1975 & 1976 & 1977 & $\frac{\text { Year }}{1978}$ & 1979 & 1980 & $19 \overline{81}$ & $\overline{1982}$ & Total & $\begin{array}{l}\text { Fra. } \\
\text { Pct } \\
(\%)\end{array}$ & $\begin{array}{c}\text { Cum. } \\
\text { Pct. } \\
(\%)\end{array}$ \\
\hline $\begin{array}{c}0-9 \\
10-19 \\
20-29 \\
30-39 \\
40-49 \\
50-59 \\
60-69 \\
70-79 \\
80-89 \\
>90 \\
\text { Total b/ }\end{array}$ & $\begin{array}{r}3,887 \\
2,221 \\
1,919 \\
1,365 \\
960 \\
391 \\
109 \\
61 \\
4 \\
8 \\
10,925\end{array}$ & $\begin{array}{r}3,793 \\
2,428 \\
2,098 \\
1,511 \\
1,026 \\
433 \\
127 \\
59 \\
6 \\
1 \\
11,482\end{array}$ & $\begin{array}{r}3,923 \\
2,339 \\
2,152 \\
1,600 \\
1,086 \\
419 \\
119 \\
68 \\
8 \\
2 \\
11,716\end{array}$ & $\begin{array}{r}4,098 \\
2,431 \\
2,097 \\
1,582 \\
1,106 \\
382 \\
95 \\
62 \\
2 \\
2 \\
11,857\end{array}$ & $\begin{array}{r}3,788 \\
2,303 \\
2,042 \\
1,457 \\
985 \\
351 \\
87 \\
51 \\
2 \\
1 \\
11,067\end{array}$ & $\begin{array}{r}3,224 \\
1,950 \\
1,589 \\
1,277 \\
887 \\
330 \\
96 \\
49 \\
2 \\
0 \\
9,402\end{array}$ & $\begin{array}{r}2,715 \\
1,724 \\
1,459 \\
1,061 \\
825 \\
279 \\
94 \\
55 \\
4 \\
1 \\
8,222\end{array}$ & $\begin{array}{r}2,125 \\
1,364 \\
1,257 \\
935 \\
742 \\
294 \\
97 \\
56 \\
1 \\
2 \\
6,873\end{array}$ & $\begin{array}{r}27,553 \\
16,765 \\
14,611 \\
10,788 \\
7,617 \\
2,879 \\
824 \\
461 \\
29 \\
17 \\
81,544\end{array}$ & $\begin{array}{r}33.79 \\
20.56 \\
17.92 \\
13.23 \\
9.34 \\
3.53 \\
1.01 \\
0.56 \\
0.04 \\
0.02 \\
100.00\end{array}$ & $\begin{array}{r}33.79 \\
54.35 \\
72.27 \\
85.50 \\
94.84 \\
98.37 \\
99.38 \\
99.94 \\
99.98 \\
100.00\end{array}$ \\
\hline
\end{tabular}

a/ U.S. Department of Transportation, Federal Railroad Administration, Office of Safety, Rail-Highway Grade-Crossing Accident/Incidents Bulletins. B.34-B. 41

b/ Excludes accidents of unknown velocities. 
Table B. 6

Summary of Objects Struck and Type of Accident for Accidents Involving U.S. Private and For-Hire Motor Carriers, 1973-1983a/

Type of Accident Total ay. b/ Pra. $\quad$ Pct. Remarks

$(\%)$

I. Nonfixed Object Collision

w/ Commercial Truck

w/ Automobile

w/ Pedestrian

w/ Bus

w/ Train

w/ Bicyclist

w/ Animal

w/ Motorcycle

w/ Other or Not Specified Subtota 1

\begin{tabular}{|c|c|c|}
\hline $\begin{array}{r}42,848 \\
143,573 \\
4,493 \\
1,477 \\
2,575 \\
1,259 \\
2,111 \\
2,680 \\
16,157 \\
217,173\end{array}$ & $\begin{array}{r}3,895 \\
13,052 \\
408 \\
134 \\
234 \\
114 \\
192 \\
244 \\
1,469 \\
19,743\end{array}$ & $\begin{array}{r}12.88 \\
43.15 \\
1.35 \\
0.44 \\
0.77 \\
0.38 \\
0.63 \\
0.81 \\
4.86 \\
65.26\end{array}$ \\
\hline
\end{tabular}

II. Fixed Object Collision

$\begin{array}{rrrr}29,476 & 2,680 & -8.86 & \text { Note } 1 \\ 246,649 & 22,423 & 74.12 & \\ 30,104 & 2,737 & 9.05 & \text { Note } 1 \\ & & & \\ 18,184 & 1,653 & 5.46 & \\ \frac{27,792}{45,976} & \frac{2,527}{4,180} & \frac{8.35}{13.82} & \end{array}$

V. Other Noncollision Accidents

Separation of Units

Fire

1,033

3,219

93.9

293

130

0.31

Cargo Loss/Spillage

1,433

$104:$

0.97

Cargo Shift $\therefore$

1,139

other or Not Specified.

Subtota 1

$\frac{3,213}{10,037}$

292

0.43

$d$

?, $10, \frac{3,213}{10,037}$

912

$0.34,:$

$\frac{0.97}{3.02}$

Noncol. Accidents Subtotal

Total Accidents

$\frac{86,117}{332,766}$

7.829

25.88

$30,251 \quad 100: 00$ a/ U.S. Department of Transportation, Bureau of Motor Carrier

b/ Based on 11 year period.

Note 1: Object distribution from California TASAS accident survey, see Table B.8. 
Table B.7

Objects Struck During California Accidents, 1975-1983a/

Object Struck

Total Avg.ㅁ $\quad \begin{aligned} & \text { Fra. } \\ & \text { Pct. }\end{aligned}$

(\%)

1. Side of Bridge Railing

2. End of Bridge Railing

3. Pier, Column, Abutment

4. Bottom of Structure (Overhead Bridge Structure)

5. Bridge End Post in Gore (01der Bridge w/Protective Is land)

10. Light or Signal Pole

11. Utility Pole

12. Pole (Type Note Stated)

13. Traffic Sign/Sign Post

14. Other Signs Not Traffic

15. Guardrai1

16. Barrier

17. \& 30. Wall (Concrete/Wood/Sound)

18. Dike or Curb

19. Traffic Island

20. Raised Bars (Delineation Bars, as Traffic Islands w/o Curb)

21. Concrete Object (Headwal1, Drop Inlet)

22. Guidepost, Culvert, Postmile Marker

23. Cut Slope or Embankment

24. Over Embankment

25. In Water

26. Drainage Ditch

27. Fence

28. Trees

29. Plants

40. Natural Material on Road

41. Temporary Barricades, Cones

42. Other Object on Road

43. Other Object off Road

44. Overturned

45. Crash Cushion

98. Unknown Object Struck

99. No Object Involved

00. Other Vehicle

$\begin{array}{rrr}9,473 & 1,053 & 0.82 \\ 1,689 & 188 & 0.15 \\ 810 & 90 & 0.07\end{array}$

$\begin{array}{lll}639 & 71 \quad 0.06\end{array}$

$\begin{array}{rrr}275 & 30.6 & 0.02 \\ 8,384 & 932 & 0.72 \\ 8,140 & 904 & 0.70 \\ 454 & 50 & 0.04 \\ 9,687 & 1,076 & 0.83 \\ 333 & 37 & 0.03 \\ 25,354 & 2,817 & 2.18 \\ 41,432 & 4,604 & 3.57 \\ 3,751 & 417 & 0.32 \\ 69,134 & 7,682 & 5.96 \\ 2,590 & 288 & 0.22\end{array}$

67

67

9,020

22,403

12,758
45

7,850

13,701

8,392

5,111

1,785

1,337

10,517

10,153

61,848

1,199

975

9,386

801,256

7.4

102

1,002

2,489

1,418

5.0

872

1,522

932

568

198

149

1,169

1,128

6,872

133

108

1,043

89,028

0.01

0.08

0.78

1.93

1.10

0.004

0.68

1.18

0.72

0.44

0.15

0.12

0.91

0.87

5.33

0.10

0.08

0.81

69.02 
Table B.7 Cont inued

\begin{tabular}{lrrr}
\hline Object Struck & Total & Avg. b/ & \multicolumn{1}{c}{$\begin{array}{r}\text { Fra. } \\
\text { Pct. } \\
(\%)\end{array}$} \\
\hline Total Primary 0bject Struck & $1,160,869$ & 128,985 & 100.00 \\
$X X . \quad$ Not Stated & 180 & 20 & N/A \\
YY. Not Appl icable & 239,655 & 26,628 & N/A \\
ZZ. Inval id Code & 164 & 18 & N/A \\
Total Accidents & $1,165,097$ & 129,455 & N/A \\
\hline
\end{tabular}

a/ TASAS Selective Record Retrieval. B.39-B.47

b/ Based on 9 year period.

N/A Not applicable. 
The CALTRANS accident data were reordered according to the accident categories defined in Table B.6. The result is Table B.8. Certain objects in Table B.8 were combined because of the similarity of these objects when considered in structural analysis calculations. The BMCS and the CALTRANS data on the object frequencies were combined to derive the probability of occurrences of the different accident scenarios.

\section{B.6 Truck Fire Duration Distributions}

The thermal response of the cask during a truck fire depends on the temperature of the fire, location of the fire relative to the cask and the duration of the fire. The type and amount of combustible materials will significantly affect the duration of a fire. Thus, the fire duration distribution will vary for different accident scenarios. For example, a fire involving a collision with a tanker truck can be expected to last longer than a fire involving a collision with an automobile or a collision with a noncombustible fixed object. To assess the probabilities of a truck cask's experiencing different thermal response levels, five fire duration distributions were developed. These distributions were associated with automobile collisions, truck collisions, collisions with fixed objects, other collisions including overturns and jackknifing, and noncollision fires. The

basis for these distributions was the fire duration program developed by Sandia. B.48 These distributions are summarized in Table 5.5 .

\section{B.7 Probability of Fire}

Not all truck accidents will involve a fire; thus it is necessary to estimate the probability of a fire given an accident. The likelihood of a fire can be expected to vary between accident scenarios. Several sources provided statistical information for several types of accidents. B.1-B.13,B.48 The probabilities of a fire given each of the different accident scenarios used in this study and listed in Table 5.9, are based on the statistics presented in the Sandia report on severe accidents. ${ }^{\text {B. }} 48$ 
Table B. 8

Objects Struck During California Accidents

Reordered According to Type of Accident, 1975-1983al

Type of Accident

Total Avg. b/ $\begin{aligned} & \text { Fra. } \\ & \text { Pct. } \\ & (\%)\end{aligned}$

Remarks

$(\%)$

I. Nonfixed Object Collision

40. Natural Material on Road

41. Temporary Barricades, Cones

42. Other Object on Road

98. Unknown Object Struck

00 . Other vehicle

Subtotal

\begin{tabular}{rrr}
1,785 & 198 & 0.15 \\
1,337 & 149 & 0.12 \\
10,517 & 1169 & 0.91 \\
975 & 108 & 0.08 \\
801,256 & $\frac{8,9028}{6028}$ & -69.02 \\
\hline 815,870 & $\frac{70.28}{9,0652}$ &
\end{tabular}

II. Fixed Object Collision

1-2. Side or End of Bridge Railing

3. Pier, Column, Abutment

4. Bottom of Structure

5. Bridge End Post in Gore

10-12. Light, Signal, Utility or Other Type Pole

13-14. Traffic Sign/Sign Post or Other Signs

15. Guardrai1

16. Barrier

17830. Wal1 (Concrete/Wood/Sound)

18-20. Dike, Curb, Traffic Island or Raised Bars

21. Concrete Object (Headwa11, Drop

22. Guidepost, Culvert, Postmile Marker

45. Crash Cushion

Subtotal

Collision Accidents Subtotal

$\begin{array}{rrrr}11,162 & 1,240 & 0.96 & \text { Note 1 } \\ 810 & 90 & 0.07 & \text { Note 2 } \\ 639 & 71 & 0.06 & \\ 275 & 30.6 & 0.02 & \end{array}$

$16,978 \quad 1,886 \quad 1.46$

$10,020 \quad 1,113 \quad 0.86$

$25,354 \quad 2,817 \quad 2.18$

$41,432 \quad 4,604 \quad 3.57$

$\begin{array}{lll}3,751 & 417 & 0.32\end{array}$

$71,791 \quad 7,977 \quad 6.18$

$\begin{array}{lll}921 & 102 & 0.08\end{array}$

$\begin{array}{lll}9,020 & 1,002 & 0.78\end{array}$

$\begin{array}{lll}1,199 & 133 & 0.10\end{array}$

$\overline{193,352} \overline{21,484} \quad \overline{16.66}$

$\overline{1,009,222} \overline{112,136} \quad \overline{86.94}$

III. Ran Off Road

23. Cut Slope or Embankment

24. Over Embankment

25. In Water

22,403

12,758

2,489

1.93

1.10

Note 3

26. Drainage Ditch

27. Fence

28. Trees

29. Plants

43. Other 0bject off Road

Subtotal

\begin{tabular}{rrr}
45 & 5.0 & 0.004 \\
7,850 & 872 & 0.68 \\
13,701 & 1,522 & 1.18 \\
8,392 & 932 & 0.72 \\
5,111 & 568 & 0.44 \\
10,153 & 1,128 & 0.87 \\
\hline 80,413 & $\frac{1,935}{6.93}$
\end{tabular}

Note 3 
Table B. 8 Continued

\begin{tabular}{|c|c|c|c|c|}
\hline Type of Accident & Total & Avg. & $\begin{array}{l}\text { Fra. } \\
\text { Pct. } \\
(\%)\end{array}$ & Remarks \\
\hline $\begin{array}{l}\text { IV. Impact with Roadbed } \\
\text { 44. Overturned }\end{array}$ & 61,848 & 6,872 & 5.33 & \\
\hline $\begin{array}{l}\text { Other Noncollision Accidents } \\
99 \text {. No Object Involved } \\
\text { Noncollision Accidents Subtotal }\end{array}$ & $\frac{9,386}{151,647}$ & $\frac{1,043}{16,850}$ & $\frac{0.81}{13.06}$ & \\
\hline Total Accidents & $1,160,869$ & 128,985 & 100.00 & \\
\hline
\end{tabular}

A11 LLNL calculations are based on static analysis. Static force is defined as ultimate static force at which complete collapse of object occurs.

a/ TASAS Selective Record Retrieval. B.39-B.47

b/ Based on 9 year period.

Note 1 Assume worst case that truck goes off bridge. Distributions of bridge heights and surfaces below bridges determined from Engineering Computer Corporation (ECC) survey in Appendix D.

Note 2 Distribution of bridge column size determined from ECC survey in Appendix D.

Note 3 Distribution of soil types and surfaces determined from ECC survey in Appendix D. 


\section{B.8 References}

B.1 1969 Accidents of Large Motor Carriers of Property, Bureau of Motor Carrier Safety, Federal Highway Administration, U.S. Department of Transportation, Washington, DC, December 1970.

B.2 1970 Accidents of Large Motor Carriers of Property, Bureau of Motor Carrier Safety, Federal Highway Administration, U.S. Department of Transportation, Washington, DC, March 1972.

B.3 1971-1972 Accidents of Large Motor Carriers of Property, Bureau of Motor Carrier Safety, Federal Highway Administration, U.S. Department of Transportation, Washington, DC, May 1974

B.4 1973 Accidents of Motor Carriers of Property, Bureau of Motor Carrier Safety, Federal Highway Administration, U.S. Department of Transportation, Washington, DC, July 1975.

B.5 1974 Accidents of Motor Carriers of Property, Bureau of Motor Carrier Safety, Federal Highway Administration, U.S. Department of Transportation, Washington, DC, 1975.

B.6 1975 Accidents of Motor Carriers of Property, Bureau of Motor Carrier Safety, Federal. Highway Administration, U.S. Department of Transportation, Washington, DC, 1976.

B.7 1976 Accidents of Motor Carriers of Property, Bureau of Motor Carrier Safety, Federal Highway Administration, U.S. Department of Transportation, Washington, DC, October 1977.

B.8 1977 Accidents of Motor Carriers: of Property, Bureau of Motor Carrier Safety, Federal Highway Administration, U.S. Department of Transportation, Washington, DC, May 1980. 
B.9 1978 Accidents of Motor Carriers of Property, Bureau of Motor Carrier Safety, Federal Highway Administration, U.S. Department of Transportation, Washington, DC, May 1980.

B.10 1979 Accidents of Motor Carriers of Property, Bureau of Motor Carrier Safety, Federal Highway Administration, U.S. Department of Transportation, Washington, DC, 1980.

B.11 1980-1981 Accidents of Motor Carriers of: Property, Bureau of Motor Carrier Safety, Federal Highway Administration, U.S. Department of Transportation, Washington, DC, August 1982.

B.12 1982 Accidents of Motor Carriers of Property, Bureau of Motor Carrier Safety, Federal- Highway Administration, U.S. Department of Transportation, Washington, DC, May 1983.

B.13 1983 Accidents of Motor Carriers of Property, Bureau of Motor Carrier Safety, Federal Highway Administration, U.S. Department of Transportation, Washington, DC, October 1984.

B.14 Summary of Motor Vehicle Accidents in the Petroleum Industry for 1977, American Petroleum Institute, Washington, DC, May 1978.

B.15 Suminary of Motor Vehicle Accidents in the Petroleum Industry for 1978, American Petroleum Institute, Washington, DC, August 1979.

B.16 Summary of Motor Vehicle Accidents in the Petroleum Industry for 1979, American Petroleum Institute, Washington, DC, June 1980.

B.17 Summary of Motor Vehicle Accidents in the Petroleum Industry for 1980, American Petroleum Institute, Washington, DC, September 1981. 
B.18 Summary of Motor Vehicle Accidents in the Petroleum Industry for 1981, American Petroleum Institute, Washington, DC, August 1982.

B.19 1957 Annual Statistical Report, Department of California Highway Patrol, Sacramento, CA, May 1958.

B.20 1958 Annual Statistical Report, Department of California Highway Patrol, Sacramento, CA, May 1959.

B.21 1959 Annual Statistical Report, Department of California Highway Patrol, Sacramento, CA, May 1960.

B.22 1960 Annual Statistical Report, Department of California Highway Patrol, Sacramento, CA, May 1961.

B.23 1961 Annual Statistical Report, Department of California Highway Patrol, Sacramento, CA, May 1962.

B.24 1962 Traffic Accident Statistics, Department of California Highway Patrol, Sacramento, CA, May 1963.

B.25 1963 Traffic Accident Statistics, Department of California Highway Patrol, Sacramento, CA, May. 1964.

B.26 1964 Traffic Accident Statistics, Department. of California Highway Patrol, Sacramento, CA, May 1:965

B.27 1965 Traffic Accident Statistics, Department of California Highway Patrol, Sacramento, CA , Apri1 1966:

B.28 1966 Report of Fatal and Injury Motor Vehicle Traffic Accidents, Department of California Highway Patrol, Sacramento, CA, July 1967. 
B.29 1967 Report of Fatal and Injury Motor Vehicle Traffic Accidents, Department of California Highway Patrol, Sacramento, CA, July 1968.

B.30 E. G. Hamilton, Single, Variable Tabulations for 1979-1981 North Carolina Accidents, University of North Carolina Highway Safety Research Center, Chapel Hi1l, NC, September 1977.

B.31 Rail-Highway Grade-Crossing Accidents/Incidents Bullet in for the Year Ended December 31, 1975, Office of Safety, Federal Railroad Administration, U.S. Department of Transportation, Washington, DC.

B.32 Rail-Highway Grade-Crossing Accidents/Incidents Bullet in for the Year Ended December 31, 1976, Office of Safety, Federal Railroad Administration, U.S. Department of Transportation, Washington, DC, December 1977.

B.33 Rail-Highway Grade Crossing Accident/Incident Bullet in No. 43, Calendar Year 1977, Office of Safety, Federal Railroad Administration, U.S. Department of Transportation, Washington, DC, July 1978.

B.34 Rail-Highway Crossing Accident/Incident and Inventory Bullet in No.-1, Calendar Year 1978, Office of Safety, Federal Railroad Administration, U.S. Department of Transportation, Washington, DC, October 1979.

B.35 Rail-Highway Crossing Accident/Incident and Inventory Bul let in No.2, Calendar Year 1979, Office of Safety, Federal Railroad Administration, U.S. Department of Transportation, Washington, DC, September 1980.

B.36 Rail-Highway Crossing Accident/Incident and Inventory Bulletin No. 3, Calendar Year 1980, Office of Safety, Federal Railroad Administration, U.S. Department of Transportation, Washington, DC, June 1981. 
B.37 Rail-Highway Crossing Accident/Incident and Inventory Bullet in No. 4, Calendar Year 1981, Office of Safety, Federal Railroad Administration, U.S. Department of Transportation, Washington, DC, June 1982.

B.38 Rail-Highway Crossing Accident/Incident and Inventory Bulletin No. 5, Calendar Year 1982, Office of Safety, Federal Railroad Administration, U.S. Department of Transportation, Washington, DC, June 1983.

B.39 TASAS Selective Record Retrieval Statewide Accident Summary for Year 1975 , State of California Department of Transportation, Sacramento, CA, October 1979.

B.40 TASAS Selective Record Retrieval Statewide Accident Summary for Year 1976, State of California Department of Transportation, Sacramento, CA, October 1979.

B.41 TASAS Selective Record Retrieval Total Statewide Accidents for Year 1977. State of California Department of Transportation, Sacramento, CA, May 1978.

B.42 TASAS Selective Record Retrieval Statewide Accident Summary for Year 1978, State of California Department of Transportation, Sacramento, CA, Apri1 1979.

B.43 TASAS Selective Record Retrieval, Summary Only, All Accidents for the Year 1978, State of California Department of Transportation, Sacramento, CA, August 1984.

B.44 TASAS Selective Record Retrieval, Summary Only, All Accidents for the Year 1979, State of California Department of Transportation, Sacramento, CA, August 1984. 
B.45 TASAS Selective Record Retrieval Statewide Accidents for Year 1981, State of California Department of Transportation, Sacramento, CA, April 1982.

B.46 TASAS Selective Record Retrieva? Statewide Summary 1982, State of California Department of Transportation, Sacramento, CA, May 1983.

B.47 IASAS Selective Record Retrieval Statewide Summary 1983, State of California Department of Transportation, Sacramento, CA, April 1984.

B.48 R. K. Clarke, et a1., Severities of Transportation Accidents, Sandia National Laboratory, Albuquerque, NM, SAND77-0001, 1977. 


\title{
APPENDIX C
}

\author{
List of Tables
}

Page

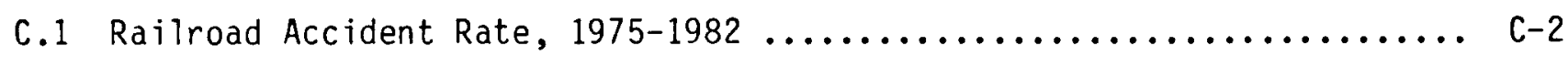

C.2 Distribution of Types of Railroad Accidents, 1975-1982 ............ C-5

C.3 Distribution of Train Velocities, Collisions, Main Line,

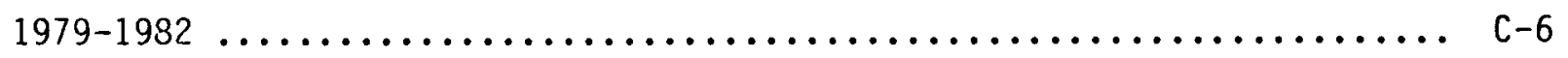

C.4 Distribution of Train Velocities, Derailments, Main Line, 1979-1982 ........................................... C-7

C.5 Distribution of Train Velocities for Rail-Highway Grade-Crossing Accidents/Incidents Involving Motor

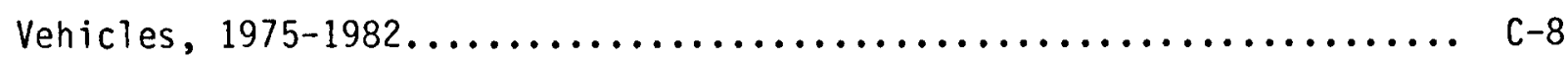

C.6 Distribution of Train Velocities, Other Accidents, Main Line,

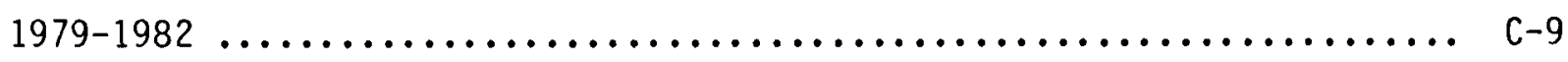

C.7 Railroad Fires Survey Results $\ldots \ldots \ldots \ldots \ldots \ldots \ldots \ldots \ldots \ldots \ldots \ldots \ldots$ C-11 


\section{APPENDIX C}

\section{Railroad Accident Data}

\section{C.1 Introduction}

This appendix summarizes both the railroad accident data which form the basis for the estimates of accident scenarios and the probability distributions used in the probabilistic analysis of future train accidents involving the transport of spent nuclear fuel. The primary sources of data were the statistical reports of railroad accidents produced by the office of Safety, Federal Railroad Administration (FRA) of the $U$. S. Department of Transportation (DOT).C.1-C.7 A Sandia report on severe accidents was the source of estimates of the probability of fire duration distributions. C. 8 Section C.2 discusses the data used to estimate the railroad accident rate and distribution of types of accidents. Section C.3 discusses the distributions of train velocity at the time of an accident; Section C.4 discusses the fire duration distribution.

\section{C.2 Railroad Accident Rate}

Federal law (49 CFR 225) requires all railroads to file monthly accident/incident reports with the office of Safety, FRA of the U. S. DOT. A railroad is defined, by regulation, as any system of surface transportation of persons or property over rails. It includes line-haul freight and passenger railroads; switching and terminal railroads; and passenger-carrying railroads including rapid transit, commuter, scenic, street, subway, elevated cable, and cog railways.

Train accidents are defined by the FRA Office of Safety as any event involving on-track railroad equipment that results in damage to railroad ontrack equipment, signals, track or track structure, and roadbed at or exceeding the dollar damage threshold. Prior to 1975, the threshold was \$750. Since 1975 this $1 \mathrm{imit}$ has been adjusted, to account for inflation, from $\$ 1750$ in 1975 to $\$ 4100$ in 1982 , the last year available for use in this study. Although initially adjusted biennially (i.e., every two years), since 1977 the adjustment has been annual. The yearly threshold limits are included in Table C. 1 . 
Table C. 1

Railroad Accident Rate, 1975-1982al

\begin{tabular}{ccccc}
\hline Year & $\begin{array}{c}\text { Train Miles } \\
\times 1000\end{array}$ & $\begin{array}{c}\text { Number } \\
\text { of } \\
\text { Accidents }\end{array}$ & $\begin{array}{c}\text { Accident } \\
\text { Rate }\end{array}$ & $\begin{array}{c}\text { Damage } \\
\text { Threshold }\end{array}$ \\
\hline 1975 & 755,033 & 8,041 & $1.06 \mathrm{E} 10^{-5}$ & $\$ 1,750.00$ \\
1976 & 774,764 & 10,248 & $1.32 \mathrm{E} 10^{-5}$ & $\$ 1,750.00$ \\
1977 & 750,042 & 10,362 & $1.38 \mathrm{E} 10^{-5}$ & $\$ 2,300.00$ \\
1978 & 751,964 & 11,277 & $1.50 \mathrm{E} 10^{-5}$ & $\$ 2,600.00$ \\
1979 & 763,429 & 9,740 & $1.28 \mathrm{E} 10^{-5}$ & $\$ 2,900.00$ \\
1980 & 717,662 & 8,451 & $1.18 \mathrm{E} 10^{-5}$ & $\$ 3,200.00$ \\
1981 & 676,216 & 5,781 & $8.55 \mathrm{E} 10^{-6}$ & $\$ 3,700.00$ \\
1982 & 573,369 & 4,589 & $8.00 \mathrm{E} 10^{-6}$ & $\$ 4,100.00$ \\
\hline Tota1 & $5,762,479$ & 68,489 & $1.19 \mathrm{E} 10^{-5}$ & \\
\hline
\end{tabular}

a/ U.S. Department of Transportation, Federal Railroad Administration, Accident/Incident Bulletins C.1-C.7 
In addition to train accidents, the FRA Office of Safety compiles and reports statistics on two related events: train incidents, and non-train incidents. Train incidents are defined as events involving on-track railroad equipment that result in the reportable death and/or injury or illness of one or more persons, but do not result in damage at or beyond the damage threshold, as defined in the previous paragraph. Non-train incidents are defined as events which result in a reportable death, injury, or $i 11$ ness arising from the operation of a railroad but not from the movement of railroad on-track equipment.

Damage to casks containing spent nuclear fuel will necessarily involve severe accidents (hence significant damage); thus; for this project, train accidents formed the basis for estimating railroad accident rates. Because of the effect of the damage threshold levels on the reported accidents, data from the period 1975 to 1982 were used to estimate the accident rate used in this study. The estimated railroad accident rate, $1.19 \times 10^{-5}$ accidents/trainmile/year, is the ratio of the number of reported accidents to the total miles for the 1975 to 1982 period.

Table C.1 presents the train mileage and number of accidents, as well as rate and damage threshold for each year during 1975 to 1982 . Train-miles, for this report, is defined as the sum of the locomotive miles, yard switching miles, and motor train miles as tabulated for each year by the FRA. The FRA defines a locomotive mile as the movement under its own power of a locomotive the distance of one mile whether coupled:or. without cars. This item covers miles run by locomotives in road services and in train and yard switching service. Switching miles are computed the thate of 6 miles/hour for the time actually engaged in such service. A motoritrain-mile is a movement under its own power of a motor train a distance of one mile

Accident severity varies between accidents; thus the level of damage that a cask might exper ience during an accident depends on the type of accident. Therefore, train accidents were subdivided into four types-collisions, derailments," rail=highway grade-crossing :accidents.) and other types of accidents. Data relevarit to this distribution; derived from the FRA reports, 
is given in Table C.2. Again, the distribution of accident types is based on the accidents during the 1975-1982 period. The important statistics are the percentages, for each type of accident, of all accidents presented in the bottom row of the table. For example, $13.41 \%$ of the train accidents were collisions.

Approximately $36 \%$ of the collisions involved derailment of at least one car. C. 8 These were grouped with the original derailment accidents. Derailment accidents were further partitioned into accident scenarios based on the events following the derailment. Accident scenarios considered included the car(s) falling over a bridge or embankment, hitting a slope or a structure, or rolling over. Categorization of derailment accidents into scenarios was not found in the literature. Thus, a distribution was developed based on similar statistics for truck accidents. This distribution is included in Fig. 2-5. To distinguish between the severity of accident scenarios, some of the accident scenarios were further subdivided, e.g., derailments involving a car's hitting a structure were subdivided into hitting small and large columns, abutments, and other accidents. Categorization of accidents into these types of scenarios was based on the Eggers study. C.9

\section{C.3 Impact Velocity Distribution}

The forces imposed on the cask at the moment of impact during an accident depend on the impact velocity of the cask or impacting object. Since impact velocity is a function of velocity and angle of impact, it is necessary to estimate the distributions of train velocities. Information on the train velocity at the time of an accident was derived, again, from the FRA data. Reliability of these statistics can be considered good since railroad locomotives are equipped with recorders to record the train's velocity prior to, during, and after the accident. The scale, although crude, is more reliable than the velocity estimates made by investigating officers at highway accident sites.

Distributions of train velocities based on accidents occurring on main lines during 1979 to 1982 are summarized in Tables C.3 through C.6 for 
Table C.2

Distribution of Types of Railroad Accidents, 1975-1982a/

\begin{tabular}{|c|c|c|c|c|c|c|}
\hline Year & $\begin{array}{c}\text { Train } \\
\text { Collisions }\end{array}$ & $\begin{array}{c}\text { Train } \\
\text { Derailments }\end{array}$ & $\begin{array}{c}\text { Rai1-Highway } \\
\text { Grade-Xing } \\
\text { Accidents }\end{array}$ & $\begin{array}{c}\text { Other } \\
\text { Accidents }\end{array}$ & $\begin{array}{c}\text { Total } \\
\text { Accidents }\end{array}$ & $\begin{array}{c}\text { Accident } \\
\text { Damage } \\
\text { Threshold }\end{array}$ \\
\hline $\begin{array}{l}1975 \\
1976 \\
1977 \\
1978 \\
1979 \\
1980 \\
1981 \\
1982 \\
\text { Tota1 } \\
\text { Fra. } \\
\text { Pct. (\%) }\end{array}$ & $\begin{array}{r}1,002 \\
1,370 \\
1,362 \\
1,476 \\
1,425 \\
1,201 \\
776 \\
572 \\
9,184\end{array}$ & $\begin{array}{r}6,328 \\
7,934 \\
8,073 \\
8,763 \\
7,482 \\
6,442 \\
4,366 \\
3,383 \\
52,771\end{array}$ & $\begin{array}{r}248 \\
352 \\
323 \\
286 \\
248 \\
246 \\
199 \\
178 \\
2,080\end{array}$ & $\begin{array}{r}463 \\
592 \\
604 \\
752 \\
585 \\
562 \\
440 \\
456 \\
4,454\end{array}$ & $\begin{array}{r}8,041 \\
10,248 \\
10,362 \\
11,277 \\
9,740 \\
8,451 \\
5,781 \\
4,589 \\
68,489\end{array}$ & $\begin{array}{l}\$ 1,750.00 \\
\$ 1,750.00 \\
\$ 2,300.00 \\
\$ 2,600.00 \\
\$ 2,900.00 \\
\$ 3,200.00 \\
\$ 3,700.00 \\
\$ 4,100.00\end{array}$ \\
\hline
\end{tabular}

a/ U.S. Department of Transportation, Federal Railroad Administration, Accident/Incident Bulletins C.1-C.7 
Table C.3

Distribution of Train Velocities, Col1isions, Main Line, 1979-1982a/

\begin{tabular}{|c|c|c|c|c|c|c|c|}
\hline \multirow[b]{2}{*}{$\begin{array}{c}\text { Velocity } \\
\text { (mph) }\end{array}$} & \multicolumn{4}{|c|}{ Year } & \multirow[b]{2}{*}{ Total } & \multirow{2}{*}{$\begin{array}{c}\text { Fra. } \\
\text { Pct. } \\
(\%)\end{array}$} & \multirow{2}{*}{$\begin{array}{c}\text { Cum. } \\
\text { Pct. } \\
(\%)\end{array}$} \\
\hline & 1979 & 1980 & $19 \overline{81}$ & 1982 & & & \\
\hline $1-10$ & 136 & 112 & 85 & 59 & 392 & 46.12 & 46.12 \\
\hline $11-20$ & 70 & 46 & 32 & 34 & 182 & 21.41 & 67.53 \\
\hline $21-30$ & 44 & 31 & 17 & 25 & 117 & 13.76 & 81.29 \\
\hline $31-40$ & 23 & 26 & 24 & 19 & 92 & 10.82 & 92.12 \\
\hline $41-50$ & 9 & 19 & 10 & 9 & 47 & 5.53 & 96.65 \\
\hline $51-60$ & 4 & 6 & 4 & 0 & 14 & 1.65 & 99.29 \\
\hline $61-70$ & 2 & 1 & 0 & 0 & 3 & 0.35 & 99.65 \\
\hline $71-80$ & 1 & 1 & 0 & 0 & 2 & 0.24 & 99.88 \\
\hline $81-90$ & 0 & 0 & 0 & 0 & & 0.00 & 99.88 \\
\hline${ }^{91} \mathrm{~b} /$ & $\frac{0}{280}$ & $\frac{1}{243}$ & $\frac{0}{172}$ & $\frac{0}{146}$ & $\frac{1}{850}$ & $\frac{0.12}{100.00}$ & 100.00 \\
\hline Totalo & 289 & 243 & $1 / 2$ & 146 & 850 & 100.00 & \\
\hline
\end{tabular}

a/ U.S. Department of Transportation, Federal Railroad Administration, Accident/Incident Bulletins C.1-C.7

b/ Excludes accidents of unknown velocities 
Table C. 4

Distribution of Train Velocities, Derailments, Main Line, 1979-1982a/

\begin{tabular}{|c|c|c|c|c|c|c|c|}
\hline \multirow[b]{2}{*}{$\begin{array}{c}\text { Velocity } \\
\text { (mph) }\end{array}$} & \multicolumn{4}{|c|}{ Year } & \multirow[b]{2}{*}{ Total } & \multirow{2}{*}{$\begin{array}{c}\text { Fra. } \\
\text { Pct. } \\
(\%)\end{array}$} & \multirow{2}{*}{$\begin{array}{c}\text { Cum. } \\
\text { Pct. } \\
(\%)\end{array}$} \\
\hline & 1979 & 1980 & 1981 & 1982 & & & \\
\hline $1-10$ & 1,736 & 1,278 & 793 & 587 & 4,394 & 40.42 & 40.42 \\
\hline $11-20$ & 841 & 634 & 416 & 359 & 2.250 & 20.70 & 61.12 \\
\hline $21-30$ & 783 & 616 & 444 & 340 & 2,183 & 20.08 & 81.20 \\
\hline $31-40$ & 325 & 333 & 238 & 195 & 1,091 & 10.04 & 91.24 \\
\hline $41-50$ & 202 & 191 & 137 & 129 & 659 & 6.06 & 97.30 \\
\hline $51-60$ & 64 & 60 & 54 & 61 & 239 & 2.20 & 99.50 \\
\hline $61-70$ & 19 & 6 & 10 & 6 & 41 & 0.38 & 99.88 \\
\hline $71-80$ & 6 & 1 & 2 & 1 & 10 & 0.09 & 99.97 \\
\hline $81-90$ & 1 & 1 & 0 & 1 & 3 & 0.03 & 100.00 \\
\hline $91 \mathrm{~h}$ & & 0 & 0 & 0 & 0 & 0.00 & 100.00 \\
\hline Total & $\overline{3,977}$ & $\overline{3,120}$ & $\overline{2,094}$ & $\overline{1,679}$ & $\overline{10,870}$ & 100.00 & \\
\hline
\end{tabular}

a/ U.S. Department of Transportation, Federal Railroad Administration, Accident/Incident Bulletins C.1-C.7

b/ Excludes accidents of unknown velocities 
Table C.5

Distribution of Train Velocities for Rai1-Highway Grade-Crossing Accidents/Incidents Involving Motor Vehicles, 1975-1982a-

\begin{tabular}{|c|c|c|c|c|c|c|c|c|c|c|c|}
\hline $\begin{array}{l}\text { Velocity } \\
\text { (mph) }\end{array}$ & 1975 & 1976 & 1977 & $\frac{\text { Year }}{1978}$ & 1979 & 1980 & 1981 & $\overline{1982}$ & Total & $\begin{array}{c}\text { Fra. } \\
\text { Pct. } \\
(\%)\end{array}$ & $\begin{array}{c}\text { Cum. } \\
\text { Pct. } \\
(\%)\end{array}$ \\
\hline $\begin{array}{c}0-9 \\
10-19 \\
20-29 \\
30-39 \\
40-49 \\
50-59 \\
60-69 \\
70-79 \\
80-89 \\
>90 \\
\text { Tota 1 b/ }\end{array}$ & $\begin{array}{r}3,887 \\
2,221 \\
1,919 \\
1,365 \\
960 \\
391 \\
109 \\
61 \\
4 \\
8 \\
10,925\end{array}$ & $\begin{array}{r}3,793 \\
2,428 \\
2,098 \\
1,511 \\
1,026 \\
433 \\
127 \\
59 \\
6 \\
1 \\
11,482\end{array}$ & $\begin{array}{r}3,923 \\
2,339 \\
2,152 \\
1,600 \\
1,086 \\
419 \\
119 \\
68 \\
8 \\
2 \\
11,716\end{array}$ & $\begin{array}{r}4,098 \\
2,431 \\
2,097 \\
1,582 \\
1,106 \\
382 \\
95 \\
62 \\
2 \\
2 \\
11,857\end{array}$ & $\begin{array}{r}3,788 \\
2,303 \\
2,042 \\
1,457 \\
985 \\
351 \\
87 \\
51 \\
2 \\
1 \\
11,067\end{array}$ & $\begin{array}{r}3,224 \\
1,950 \\
1,587 \\
1,277 \\
887 \\
330 \\
96 \\
49 \\
2 \\
0 \\
9,402\end{array}$ & $\begin{array}{r}2,715 \\
1,729 \\
1,459 \\
1,061 \\
825 \\
279 \\
94 \\
55 \\
4 \\
1 \\
8,222\end{array}$ & $\begin{array}{r}2,125 \\
1,364 \\
1,257 \\
935 \\
742 \\
294 \\
97 \\
56 \\
1 \\
2 \\
6,873\end{array}$ & $\begin{array}{r}27,553 \\
16,765 \\
14,611 \\
10,788 \\
7,617 \\
2,879 \\
824 \\
461 \\
29 \\
17 \\
81,544\end{array}$ & $\begin{array}{r}33.79 \\
20.56 \\
17.92 \\
13.23 \\
9.34 \\
3.53 \\
1.01 \\
0.56 \\
0.04 \\
0.02 \\
100.00\end{array}$ & $\begin{array}{c}33.79 \\
54.35 \\
72.27 \\
85.50 \\
94.84 \\
98.37 \\
99.38 \\
99.94 \\
99.98 \\
100.00\end{array}$ \\
\hline
\end{tabular}

a/ U.S. Department of Transportation, Federal Railroad Administration, Office of Safety, Rai1-Highway Grade-Crossing Accident/Incidents Bulletins ${ }^{B} .34-B .41$

b/ Excludes accidents of unknown velocities 
Table C.6

Distribution of Train Velocities, Other Accidents, Main Line, 1979-1982a/

\begin{tabular}{|c|c|c|c|c|c|c|c|}
\hline \multirow[b]{2}{*}{$\begin{array}{l}\text { Velocity } \\
\text { (mph) }\end{array}$} & \multicolumn{4}{|c|}{ Year } & \multirow[b]{2}{*}{ Tota 1} & \multirow{2}{*}{$\begin{array}{l}\text { Fra. } \\
\text { Pct. } \\
\text { (\%) }\end{array}$} & \multirow{2}{*}{$\begin{array}{l}\text { Cum. } \\
\text { Pct. } \\
(\%)\end{array}$} \\
\hline & 1979 & 1980 & 1981 & $\overline{1982}$ & & & \\
\hline $1-10$ & 83 & 83 & 60 & 59 & 285 & 17.59 & 17.59 \\
\hline $11-20$ & 73 & 46 & 53 & 56 & 228 & 14.07 & 31.67 \\
\hline $21-30$ & 104 & 93 & 59 & 59 & 315 & 19.44 & 51.11 \\
\hline $31-40$ & 89 & 104 & 58 & 63 & 314 & 19.38 & 70.49 \\
\hline $41-50$ & 72 & 65 & 64 & 61 & 262 & 16.17 & 86.67 \\
\hline $51-60$ & 35 & 38 & 26 & 23 & 122 & 7.53 & 94.20 \\
\hline $61-70$ & 13 & 16 & 7 & 13 & 49 & 3.02 & 97.27 \\
\hline $71-80$ & 7 & 9 & 14 & 7 & 37 & 2.28 & 99.51 \\
\hline $81-90$ & 0 & 1 & 3 & 2 & 6 & 0.37 & 99.88 \\
\hline 91 & 0 & 0 & 0 & 2 & & 0.12 & 100.00 \\
\hline Totaj & $\overline{476}$ & $\overline{455}$ & $\overline{344}$ & $\overline{345}$ & $\overline{1,620}$ & 100.00 & \\
\hline
\end{tabular}

a/ U.S. Department of Transportation, Federal Railroad Administration, Accident/Incident Bulletins C.1-C.7

b/ Excludes accidents of unknown velocities 
collisions, derailments, highway grade-crossing accidents, and other accidents respectively. The percentages and cumulative percentages shown in the bottom two rows of each table were used to estimate probability distributions for train velocities. The estimation procedure is discussed in Appendix $G$.

\section{C.4 Probabilities of Fire and Fire Duration Distributions for Train Accidents}

There is very little useful data regarding the occurrence of fires and the properties of the fire, such as duration, given a train accident. Table C.7 presents the results of surveys of train fires, compiled by the National Fire Protection Association for the years 1976-78 and 1982-83.C.10,C.11 over this time, for the railroads surveyed, approximately $1.24 \%$ of all railroad fires occur as a result of a collision or derailment. This is interpreted probabilistically as the (conditional) probability, given a fire, that the cause of the fire is either a collision or derailment. On the other hand, the probability of interest for this study is the (conditional) probability, given a collision (or a derailment), that a fire also occurs. To derive the latter probability from the former, it is necessary to have some estimate of the probability of a fire given an accident. The necessary data to estimate this probability was not found. Therefore, the Sandia study estimate of the probabilities of a fire's occurring, given an accident scenario was used. C. 8

No information was found regarding the duration of fires resulting from train accidents. Therefore, the simulated estimates for fire duration as developed in the Sandia study were used. C.8 
Table C.7

Railroad Fires Survey Results a/

Year

Pct.of

$1976 \quad 1977 \quad \frac{1978}{1982} 1983$ Total Avg. Total (\%)

Class I Railroads Surveyed:

$22 \quad 16 \quad 16$

NA

$\begin{array}{llll}\text { Trackage Surveyed (miles): } & 129,382 & 116,405 & 94,509 \\ \text { Total Class I Trackage (miles) : } & 240,250 & 236,351 & 233,956\end{array}$

Percentage of Total (\%):

$53.85 \quad 49.25 \quad 40.40$

NA NA 340,296

NA. NA 710,557

N/A N/A N/A

$\begin{array}{rr}19.0 & N / A \\ 113,432 & N / A \\ 236,852 & N / A \\ 47.89 & N / A\end{array}$

Number of Fires due to Operations and Transportation

Collisions and Derailments

Brake Shoe Sparks

Electrical Components

Engine Exhaust Sparks

18

198

24

157

34

35

354

Car and Van Heaters

Fuses

Hot Journal Boxes

Materials in Transit

I. C. Engines

other

Subtotal

\begin{tabular}{rrrr}
14 & 19 & 12 & 87 \\
115 & 188 & 63 & 721 \\
136 & 53 & 42 & 300 \\
17 & 120 & 195 & 709 \\
12 & 3 & 17 & 76 \\
7 & 7 & 5 & 42 \\
19 & 11 & 11 & 94 \\
22 & 5 & 8 & 118 \\
14 & 25 & 8 & 80 \\
58 & 82 & 78 & 303 \\
\hline 414 & -513 & $-\frac{139}{439}$ & $\overline{2,530}$
\end{tabular}

$17.4 \quad 1.24$

$144.2 \quad 10.30$

$60.0 \quad 4.28$

$141.8 \quad 10.12$

$15.2 \quad 1.09$

$8.4 \quad 0.60$

$18.8 \quad 1.34$

$23.6 \quad 1.68$

$16.0 \quad 1.14$

$60.6 \quad 4.33$

$-506.0-\frac{4.13}{36.13}$

Number of Fires due to Maintenances and Services

Smoking

Electrical

Flammable Liquids

Heaters and Appliances

Burning on Right-of-Way

Spontaneous Ignition

Welding, Cutting, Brazing

other

Subtotal
23

28

3

72

11

18

74

41
$-270 \quad 43$
262
20

26

10 :

69

12

27

55

\section{s}

13

$11 \quad 19$

$\begin{array}{lll}11 & 19 \quad 86\end{array}$

86
124

29

317

149

89

$\begin{array}{rrrr}64 & 59 & 63 & 315 \\ 26 & 29 & 24 & 163 \\ -220 & \frac{29}{333} & \frac{187}{1,272}\end{array}$

\begin{tabular}{rrrr}
64 & 59 & 63 & 315 \\
26 & 29 & 24 & 163 \\
\hline .220 & $\frac{29}{333}$ & -187 & 1,272
\end{tabular}

Number of Fires due to Outside or Undetermined Causês

Exposure Fires

Lightning and Storms

Trespassing (including Arson)

other

Undetermined Causes

Subtota 1

Grand Total

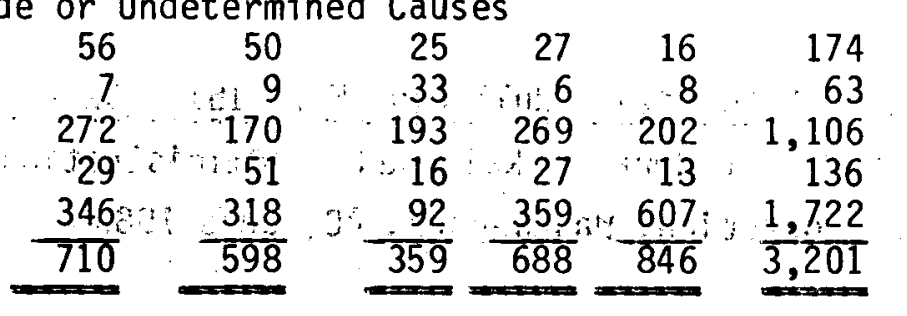

174

63

136

$\frac{1,7,22}{3,201}$

$1,756^{\circ} \quad 1,248 \quad 9931,534,1,472 \quad 7,003^{\circ}$
$17.2 \quad 1.23$

$24.8 \quad 1.77$

$5.8 \quad 0.41$

$63.4 \quad 4.55$

$29.8 \quad 2.13$

$17.8 \quad 1.27$

$63.0 \quad 4.50$

$32.6 \quad 2.33$

$\frac{32.6}{254.418 .16}$

a/ National Fire Protection Association C.8,C.9

$\bar{N} A$ Information not available at time of table preparation

N/A Not applicable

$$
\text { C-11 }
$$




\section{C.5 References}

C.1 Accident/Incident Bullet in No. 145, Calendar Year 1976, Office of Safety, Federal Railroad Administration, U.S. Department of Transportation, Washington, DC, December 1977.

C.2. Accident/Incident Bulletin No. 146, Calendar Year 1977, Office of Safety, Federal Railroad Administration, U.S. Department of Transportation, Washington, DC, August 1978.

C.3 Accident/Incident Bulletin No. 147, Calendar Year 1978, office of Safety, Federal Railroad Administration, U.S. Department of Transportation, Washington, DC, October 1979.

C.4 Accident/Incident Bulletin No. 148, Calendar Year 1979, office of Safety, Federal Railroad Administration, U.S. Department of Transportation, Washington, DC, July 1980.

C.5 Accident/Incident Bulletin No. 149, Calendar Year 1980, Office of Safety, Federal Railroad Administration, U.S. Department of Transportation, Washington, DC, June 1981.

C.6 Accident/Incident Bulletin No. 150, Calendar Year 1981, Office of Safety, Federal Railroad Administration, U.S. Department of Transportation, Washington, DC, June 1982.

C.7 Accident/Incident Bullet in No. 151, Calendar Year 1982, Office of Safety, Federal Railroad Administration, U.S. Department of Transportation, Washington, DC, June 1983.

C.8 R. K. Clarke, et al., Severities of Transportation Accidents, Sandia National Laboratory, Albuquerque, NM, SAND77-0001, 1977. 
C.9 P. Eggers, Severe Rail and Truck Accidents: Toward a Definition of Brounding Environments for Transportation Packages, U.S. Nuclear Regulatory Commission, Washington, DC, NUREG/CR-3499, 1983.

C.10 Fire Protection Handbook, 15th Edition, G. P. Mckinnon, Ed., National Fire Protection Association, Quincy, MA, 1981.

C.11 R. L. Best, National Fire Protection Association, Railroad Section, Quincy, MA, private communication, 1985. 


\section{APPENDIX D}

\section{List of Figures}

Page

D-1 Single column bent bridge structure configuration $\ldots \ldots \ldots \ldots \ldots \ldots \ldots$ D-8

D-2 Multi-column bent bridge structure configuration $\ldots \ldots \ldots \ldots \ldots \ldots \ldots . . \ldots$ D-9

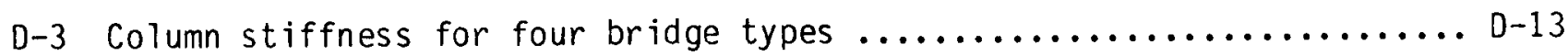

D-4 Force-displacement curves for single, square bridge columns ........ D-16

D-5 Force-displacement curves for single, rectangular bridge columns ..... D-17

D-6 Force-displacement curves for multi, square bridge columns .......... D-18

D-7 Force displacement curves for multi, rectangular bridge columns ...... 0 -19

List of Tables

Page

D.1 Type of Soil Adjacent to Interstate 5 from San Diego County/Orange County Line to Los Angeles County/Kern County Line ..... D-3

D.2 Type of Soil Adjacent to Interstate 80 from Davis, California

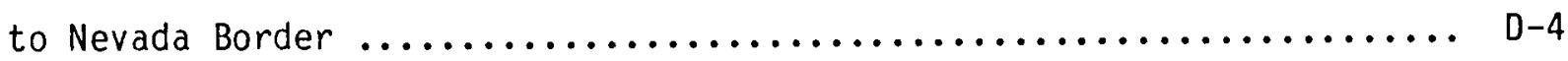

D.3 Type of Surface below Bridges on Interstate 80 from Davis, California to Nevada Border .......................... D-5

D.4 Bridges Along Interstate 5 from San Diego County/Orange County Line to Los Angeles County/Kern County Line Classified by Height................................. D-7

D.5 Twenty-Four Representative Column Configurations for Calculating

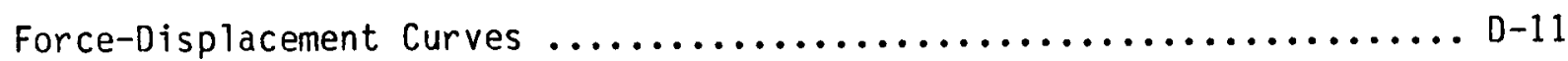


APPENDIX D

Highway Survey Data and Bridge Column Properties a/

\section{D.1 Introduction}

One important element in calculating shipping cask responses to accident loads is object hardness. When a shipping cask strikes a soft surface such as sand, the response of the cask is much less than when striking a hard object such as a concrete column. This appendix presents the data and evaluation results on two major subjects related to hard objects:

1) Statistical data on the total number of bridges, bridge heights, and surface conditions adjacent to highways, and below bridges,

2) The characteristics of bridge columns.

\subsection{Survey}

\section{D.2.1 Surface Conditions Adjacent to Highways and below Bridges}

The hardness of earth surfaces adjacent to highways can vary over a wide range. This variability can have a significant effect on the loadings that could be imposed on a cask or any other impacting object. The water and land (hard rock, soft rock/hard soil, and tillable soil) distribution along proposed spent fuel shipment routes between the east coast and west coast was initially estimated using agricultural soil survey data and geological highway maps for the United States. D.3,D.4 The initial distributions estimated from these sources were considered to be indicative of the types of surfaces which could be impacted along highways in the various regions of the United States. However, since highway construction and landscaping can greatly affect the adjacent surroundings, the initial distributions were used to select representative portions of Interstates 5 and 80 in California to

a/ The Engineering Computer Corporation (ECC) was the subcontractor that
performed the highway surveys and bridge column analyses. 
perform detailed highway surveys and to establish final distributions along highways.

A 133-mile portion of Interstate 5 was selected for the study. This portion of highway starts from the borderline between San Diego County and Orange County and ends at the borderline between Kern County and Los Angeles county. This portion of highway contains 20 miles of suburban, 50 miles of city, and 63 miles of rural area. The terrain which this portion of the highway crosses is essentially flat for 70 miles, rolling hills for 41 miles, and mountains for 22 miles. The types of earth adjacent to the highway were classified into three groups: tillable soil, non-tillable soil, and hard rock. The survey was performed by viewing the California Department of Transportation (CALTRANS) photo log. The result of the survey is summarized in Table 0.1. Although the highway crossed the Santa Susana Mountains, no hard rock, such as granite, was identified in the survey.

A similar highway survey of earth types adjacent to 122 miles of the roadway along a section of Interstate 80 from Davis, California, to the Nevada border was then performed. This section of Interstate 80 crosses the Sierra where numerous outcroppings of granite rock occur. The result of the soil survey is summarized, in Table 0.2. The survey also included the types and frequencies of surfaces that could be impacted below a bridge. These surfaces were classified into four categories: roadbeds, railbeds, water, and earth. The result of the survey is summarized in Table 0.3 .

\section{D.'2.2 Highway Bridges}

The same portion of Interstate 5 was used to compile statistical data on the number of bridges, bridge heights, and the size of columns. A two-step procedure was used in compiling data.

Step 1: View the California Department of Transportation (CALTRANS) photo $\log$ (a motion picture of the roadway as viewed by a motorist). Estimate the bridge column sizes and the number of bridges. 
Table D.1

Type of Soil Adjacent to Interstate 5 from San Diego County/Orange County Line to Los Angeles

County/Kern County Line

\begin{tabular}{|c|c|c|c|c|}
\hline \multirow[b]{2}{*}{ County } & \multicolumn{4}{|c|}{$\begin{array}{c}\text { Adjacent So } 11 \text { Type } \\
\text { (wiles) }\end{array}$} \\
\hline & Tillable & Nontiflable & Hard Rock & Total \\
\hline $\begin{array}{l}\text { Orange } \\
\text { Los Angeles } \\
\text { Total }\end{array}$ & $\begin{array}{r}44.27 \\
62.65 \\
16.39 \\
123.31\end{array}$ & $\begin{array}{l}0.12 \\
5.80 \\
\frac{3.60}{9.52}\end{array}$ & $\begin{array}{l}0 \\
0 \\
0 \\
\overline{0}\end{array}$ & $\begin{array}{r}44.39 \\
68.45 \\
19.99 \\
132.83\end{array}$ \\
\hline
\end{tabular}


Table 0.2

Type of Soil Adjacent to Interstate 80 from
Davis, California to Nevada Border

\begin{tabular}{|c|c|c|c|c|}
\hline \multirow[b]{2}{*}{ County } & \multicolumn{4}{|c|}{$\begin{array}{c}\text { Adjacent Soil Type } \\
\text { (miles) }\end{array}$} \\
\hline & Tillable & Nontillable & Hard Rock & Total \\
\hline Yolo & 2 & 0 & 0 & 2 \\
\hline Sacramento & 18 & 0 & 0 & 18 \\
\hline Placer & 60 & 2 & 3 & 65 \\
\hline Nevada & 29 & 6 & 0 & 35 \\
\hline Sierra & 1 & 1 & $\underline{0}$ & 2 \\
\hline Total & 110 & $\overline{9}$ & $\overline{3}$ & $\overline{122}$ \\
\hline
\end{tabular}

a/ 122-mile highway through mountainous terrain from Davis, California, to the California-Nevada borderline. 
Table 0.3

Type of Surface below Bridges on Interstate, 80 from Davis, California to Nevada Borderal

\begin{tabular}{lccccc}
\hline & \multicolumn{5}{c}{$\begin{array}{c}\text { Surface below Each Bridge } \\
\text { (bridge totals) }\end{array}$} \\
\cline { 2 - 5 } County & Road & River & Earth & RaiTroad & TotaT \\
\hline Yolo & 1 & 1 & 0 & 1 & 3 \\
Sacramento & 7 & 0 & 0 & 1 & 8 \\
Placer & 22 & 5 & 1 & 1 & 29 \\
Nevada & 12 & 6 & 0 & 1 & 19 \\
Sierra & 0 & 0 & 0 & $\frac{0}{4}$ & $\frac{0}{59}$ \\
\multicolumn{1}{c}{ Total } & $\frac{1}{42}$ & 12 & $\frac{1}{4}$ & & \\
\hline
\end{tabular}

a/ 122-mile highway through mountainous terrain from Davis, California, to the California-Nevada border line. 
Step 2: Review the general plans for several of the bridges to confirm the column sizes identified by visual inspection through the photo log and to obtain bridge heights.

Table 0.4 presents the result of the survey for the total number of bridges tabulated according to the bridge heights. Along the 133-mile roadway, 121 bridges were counted. Only 3 bridges exceed 50 feet in height. The rate is approximately $0.91 \mathrm{bridges} / \mathrm{mi}$ le.

While collecting data about the bridge rate, information was also collected on all of California state and interstate highways. The total number of bridges in California is 12,574 and the miles of state and interstate highways is 15,183 . This is very close to the detailed survey results of Interstate Highway 5.

\section{D.3 Bridge Column Structural Characteristics}

In order to estimate the response of a cask when impacting a bridge column, it is necessary to determine the level of hardness for that particular column. The level of hardness is normally represented by the forcedisplacement curve.

This subsection describes the approach used to develop the forcedisplacement curves for various column designs and the results of the detailed sensitivity study.

From the survey of Interstate 5, two typical bridge constructions are commonly seen along interstate highways: single-column bent bridge and multicolumn bent bridge, as shown in Figs. $D-1$ and $D-2$ respectively. Most of the bridge columns are either square or rectangular. Bridge span lengths and column bent widths vary from bridge to bridge. Since more than 12,000 bridges exist on state and interstate highways in California, estimating the column force-displacement curve for each bridge is a very complex task. In order to control the task, 13 different sizes of column cross-sections from $1 \mathrm{ft} \times 1 \mathrm{ft}$ to $4 \mathrm{ft} \times 64 \mathrm{ft}$ were selected. In combination with the number of bents, a total of 24 column configurations were selected for sensitivity study in 
Table 0.4

Bridges Along Interstate 5 from San Diego

County/Orange County Line to Los Angeles Cqunty/Kern

County Line Classified by Height

\begin{tabular}{|c|c|c|c|c|c|c|c|c|c|}
\hline \multirow[b]{2}{*}{ County } & \multicolumn{9}{|c|}{$\begin{array}{l}\text { Bridge Height } \\
(\mathrm{ft})\end{array}$} \\
\hline & $0-10$ & $11-20$ & $21-30$ & $31-40$ & $41-50$ & $51-60$ & $61-70$ & $71-80$ & $81-90$ \\
\hline Orange & $\begin{array}{l}3 \\
1\end{array}$ & $\begin{array}{l}4 \\
3\end{array}$ & $\begin{array}{r}16 \\
7\end{array}$ & 4 & & & & & \\
\hline $\begin{array}{l}\text { Los Angeles } \\
\text { Total }\end{array}$ & 1 & $\begin{array}{r}3 \\
7 \\
-5 \\
22\end{array}$ & $\begin{array}{l}17 \\
16 \\
18 \\
74\end{array}$ & $\begin{array}{l}6 \\
2 \\
1 \\
14\end{array}$ & $\begin{array}{l}2 \\
\frac{1}{3}\end{array}$ & $\frac{1}{1}$ & $\overline{1}$ & & $\frac{1}{1}$ \\
\hline
\end{tabular}

Total Mileage $=133$ miles

Total Bridges $=121$

a/ Each set (left/right pair, on/off ramps, etc.) counts only once. Special truck lanes in northern Los Angeles County are not counted. 


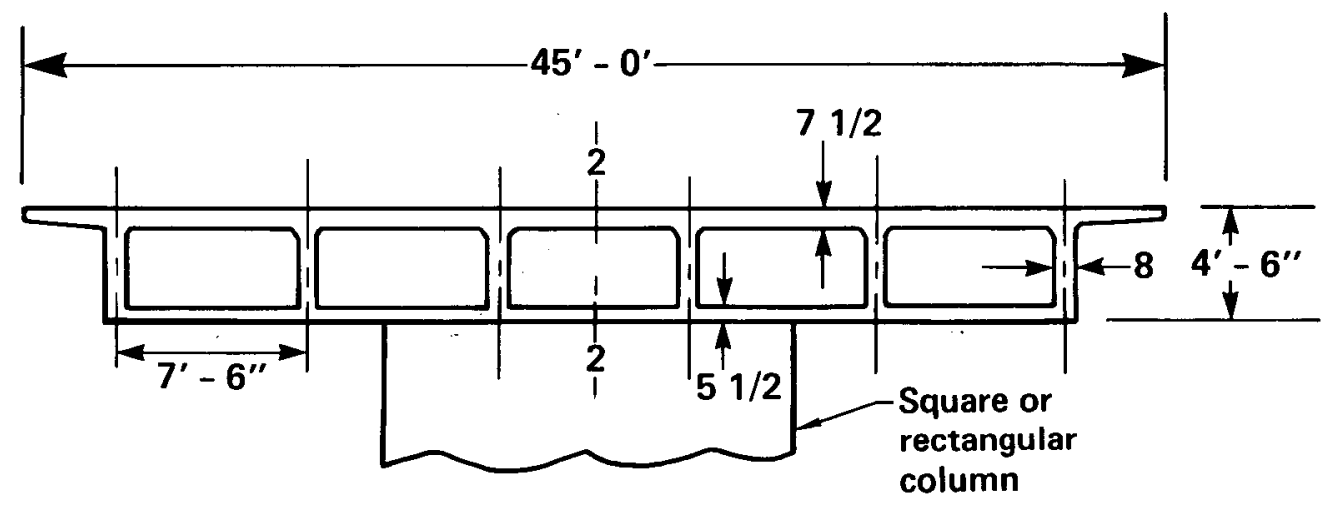

Section

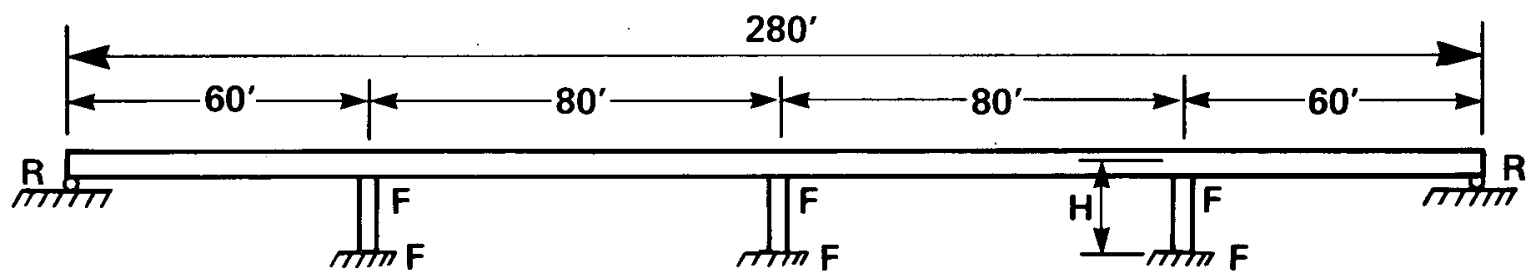

Elevation

Figure D-1 Single column bent bridge structure configuration. 

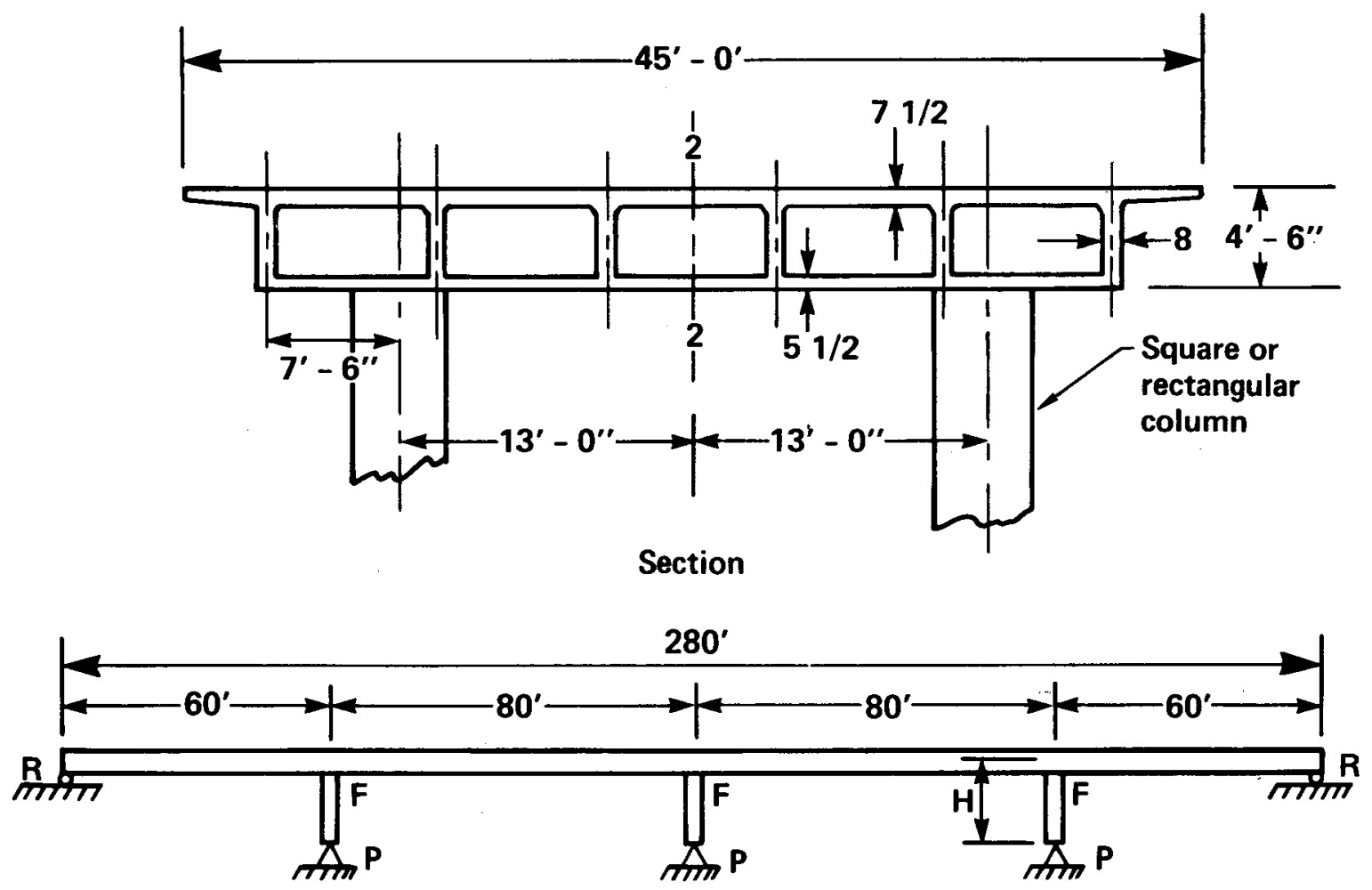

Elevation

Figure D-2 Multi-column bent bridge structure configuration. 
developing force-displacement curves. Table 0.5 lists all column configurations selected for the sensitivity study. Some of the column dimensions, such as $32 \mathrm{ft} \times 32 \mathrm{ft}, 16 \mathrm{ft} \times 16 \mathrm{ft}$, are not real structures. The inclusion of these dimensions in the analytical study is to help develop a set of continuous curves.

A11 24 different configurations were categorized into four groups according to the shape of columns, i.e., square or rectangular, and number of bents, i.e., single-bent or multi-bent. These four groups formed the four basic cases for the sensitivity study as listed below and indicated in Table D.5.

Case A: Bridges with square columns and single-column bents.

Case B: Bridges with rectangular columns and single-column bents.

Case C: Bridges with square columns and multi-column bents.

Case D: Bridges with rectangular columns and multi-column bents.

\section{D.4 Column Stiffness Sensitivity Study}

The sensitivity study assumed that a shipping cask would strike the bridge column 4 feet above the rough surface, or 6 feet above the bottom of the column (bottom of pier). The study includes column heights of 20 feet and 30 feet.

For a single-bent column, the assumption is that the bottom of the column is pinned and the top of the column is fixed. A normalized static force of 1,000 kips is applied at 6 feet above the bottom of the column to represent the impact force of the shipping cask. Deformation at the point of impact is calculated for all column sizes of both cases $A$ and $B$. The stiffness of the column is determined from the appl ied force and calculated deformation.

For the multi-bent configuration, the bridge is assumed to be a four-span bridge, which is most commonly seen along interstate highways. A beam-element 
Table 0.5

Twenty-Four Representative Column Configurations for Calculating Force-Displacement Curves

\begin{tabular}{|c|c|c|c|}
\hline Class & Number of Bents & Shape of Column & $\begin{array}{c}\text { Column Size } \\
\text { (cross-section) }\end{array}$ \\
\hline$A$ & Single & Square & $\begin{array}{r}1 \mathrm{ft} \times 1 \mathrm{ft} \\
2 \mathrm{ft} \times 2 \mathrm{ft} \\
4 \mathrm{ft} \times 4 \mathrm{ft} \\
8 \mathrm{ft} \times 8 \mathrm{ft} \\
16 \mathrm{ft} \times 16 \mathrm{ft} \\
32 \mathrm{ft} \times 32 \mathrm{ft}\end{array}$ \\
\hline B & Single & Rectangular & $\begin{array}{l}4 \mathrm{ft} \times 1 \mathrm{ft} \\
4 \mathrm{ft} \times 2 \mathrm{ft} \\
4 \mathrm{ft} \times 8 \mathrm{ft} \\
4 \mathrm{ft} \times 16 \mathrm{ft} \\
4 \mathrm{ft} \times 32 \mathrm{ft}\end{array}$ \\
\hline$\cdot$ & Multi & Square & $\begin{array}{r}1 \mathrm{ft} \times 1 \mathrm{ft} \\
2 \mathrm{ft} \times 2 \mathrm{ft} \\
4 \mathrm{ft} \times 4 \mathrm{ft} \\
8 \mathrm{ft} \times 8 \mathrm{ft} \\
16 \mathrm{ft} \times 16 \mathrm{ft} \\
32 \mathrm{ft} \times 32 \mathrm{ft}\end{array}$ \\
\hline$D$ & Multi & $\begin{array}{l}\text { Rectangular } \\
\because: \text { sur. }\end{array}$ & $\begin{array}{l}4 \mathrm{ft} \times 1 \mathrm{ft} \\
4 \mathrm{ft} \times 2 \mathrm{ft} \\
4 \mathrm{ft} \times 8 \mathrm{ft} \\
4 \mathrm{ft} \times 16 \mathrm{ft} \\
4 \mathrm{ft} \times 32 \mathrm{ft} \\
4 \mathrm{ft} \times 64 \mathrm{ft}\end{array}$ \\
\hline
\end{tabular}

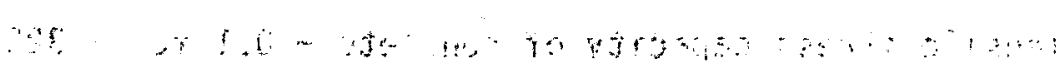

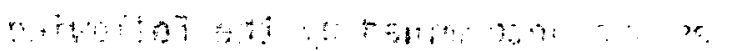

$\therefore \quad \therefore$ 
model along the bridge roadway was developed to represent the bridge superstructure. The bridge is assumed to be pinned at both ends. At each pier location, the multiple-bent column configuration is modeled by a space frame pinned at the bottom of the frame structure. The combined bridge superstructure and column space frames formed the total bridge design. A normalized static force of 1,000 kips is applied 6 feet above the bottom of the column. The deformation at the point of impact is calculated by the Structural Analysis Program 6 (SAP6) program. The force-deformation relationship is used to determine the stiffness of the columns for each pier. This process is performed on all the column sizes for cases $C$ and $D$.

Figure D-3 presents the results of this sensitivity study.

\section{D.5 Force-Displacement Curve}

The force-displacement curve was developed by following similar procedures to those described in the stiffness calculation. The same four groups (Cases A through D) were used. All the column sizes given in Table 0.5 were included in the sensitivity study. During this exercise, column capacity was considered in resisting axial force, shear force, and bending moment. The angle of impact to the column was also considered. The impact was analyzed for every $15^{\circ}$ angle. The smallest column capacity for resisting impact at the various impact angles is selected to represent the column capacity. In estimating column capacity, the following assumptions were made to simplify the problem:

1. Vertical reinforcement is $2 \%$

2. $f c^{\prime}=3,250$ psi

3. Tensile stress capacity of concrete $=0.1 \mathrm{fc}^{\prime}=325$ psi

4. Ties are determined by the following formula

$$
A_{s h}=0.30 S_{t} h_{c} \frac{f c^{\prime}}{f_{y}}\left(\frac{A g}{A c}-1\right)
$$


Case A: Square column, single-bent, column size $D^{\prime} \times D^{\prime}$

Case B: Rectangular column, single-bent, column size $4^{\prime} \times D^{\prime}$

Case C: Square column, multi-bent, column size $D^{\prime} \times D^{\prime}$

Case D: Rectangular column, multi-bent, column size $4^{\prime} \times D^{\prime}$

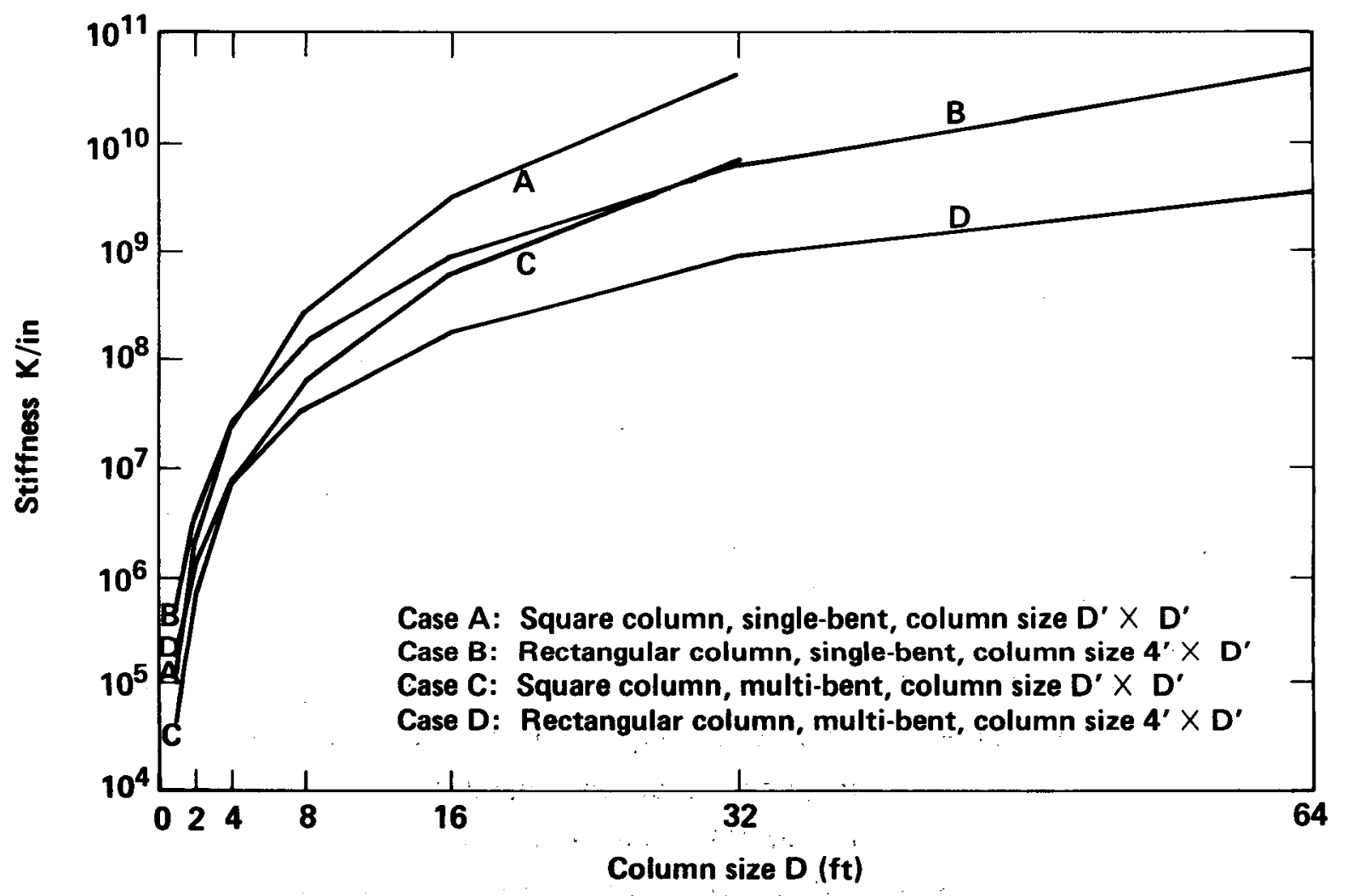

Figure D-3 Column stiffness for four bridge types. 
or

$$
A_{s h}=0.12 S_{t} h_{c} \frac{f c^{\prime}}{f_{y}}\left(0.5+1.25 \frac{P e}{f c^{\top} A g}\right)
$$

where

$$
\begin{aligned}
A_{s h} & =\text { area of transverse hoop bar, } \mathrm{ft}^{2} \\
\mathrm{fC}^{\prime} & =\text { specified compressive strength of concrete, psi } \\
h_{c} & =\text { total depth of shear head cross-section, } \mathrm{ft} \\
\mathrm{S}_{\mathrm{t}} & =\text { vertical spacing of ties, ft } \\
\mathrm{Pe} & =\text { maximum design axial load, lbs } \\
\mathrm{Ag} & =\text { gross area of section, } \mathrm{ft}^{2} \\
\mathrm{Ac} & =\text { area of concrete enclosed by tie, } \mathrm{ft}^{2} \\
\mathrm{f}_{y} & =\text { specified yield strength of re-bar, psi }
\end{aligned}
$$

5. Height of column is 20 feet.

6. Distance from the face of concrete to the center of vertical re-bars is 3 inches.

7. Moment magnification due to slenderness is ignored.

8. $P-\Delta$ effect is ignored.

From assumption number 3 , an axial force capacity was calculated for each different columin size (cross section). For the flexural capacity, the Reinforced Column (RECOL) computer code was used to estimate column strength at yield point. These axial and flexural capacities of a column are combined with the results from the stiffness calculation as generated in the bridge model by using the SAP6 computer code to correlate the force-displacement relationship for each different column size.

These force-displacement relation curves used to relate the column yield force and displacement at the location of impact are listed in 
Figs. D-4 through D-7 for all the column sizes listed in Table 0.5. The possible dominant failure modes are identified in each curve. For example, for each column size, we identify whether a plastic hinge or a sudden shear failure occurs first. The shear capacity for a column is based on the equation

$$
v_{u}=2\left(f c^{\prime}\right)^{1 / 2} b d+\frac{A_{s h} f_{y} d}{s}
$$

where

$\mathrm{b}=$ width of compression face, $\mathrm{ft}$

$d=$ distance from extreme compression fiber to centroid of tension reinforcement, $\mathrm{ft}$

$s=$ tie spacing, $f t$. 


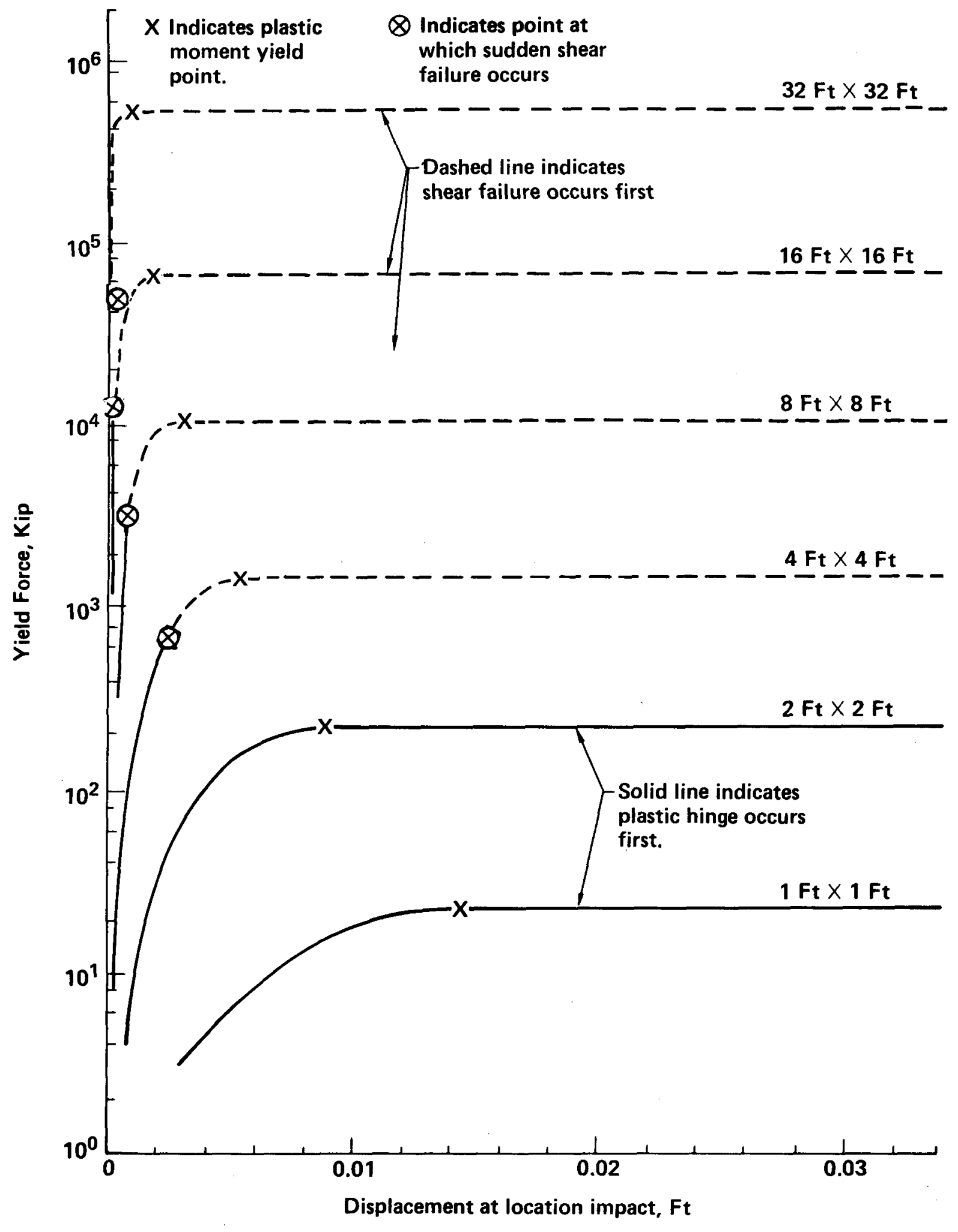

Figure D-4 Force-displacement curves for single, square bridge columns. 


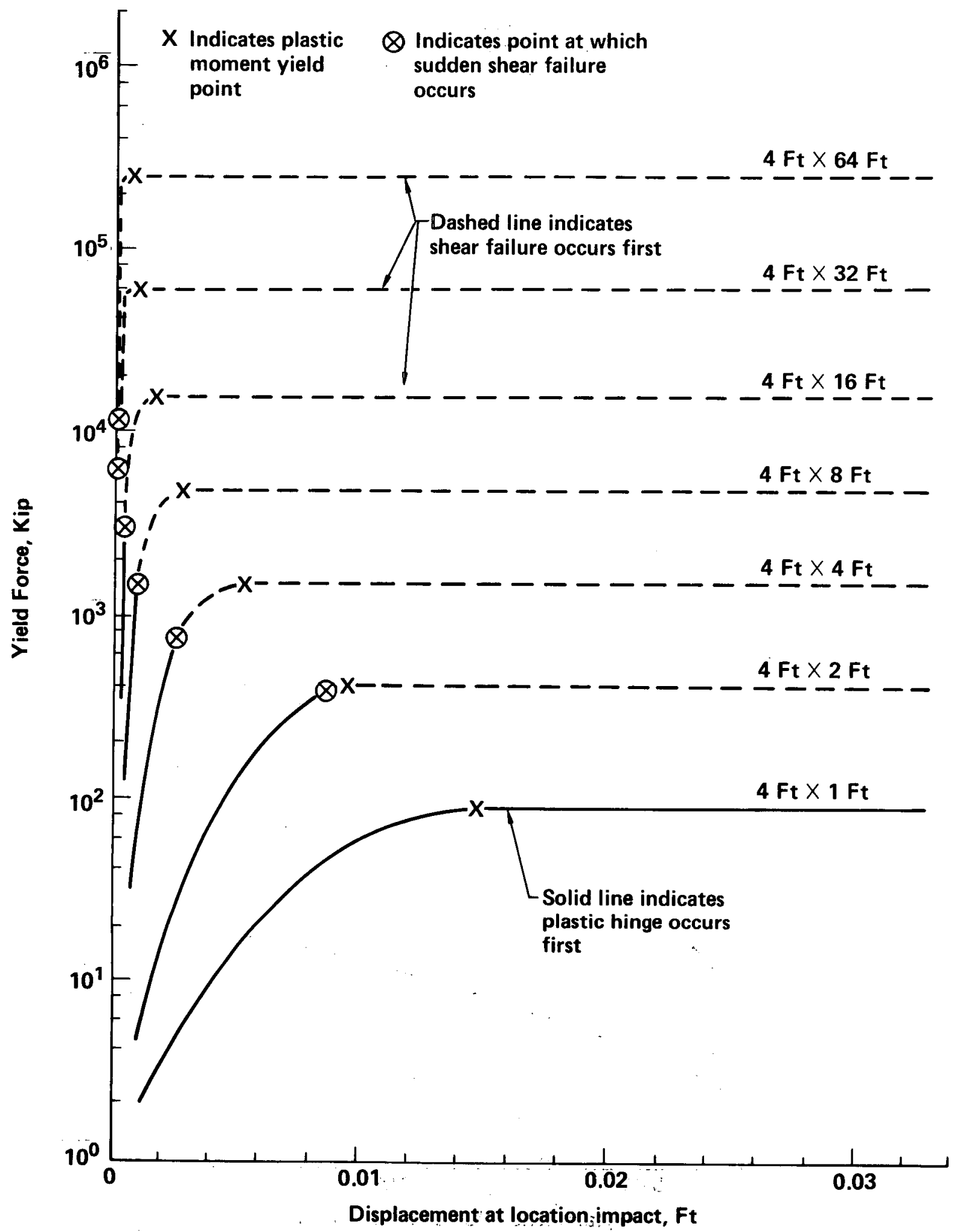

Figure 0-5 Force-displacement curves for single, rectangular bridge columns. 


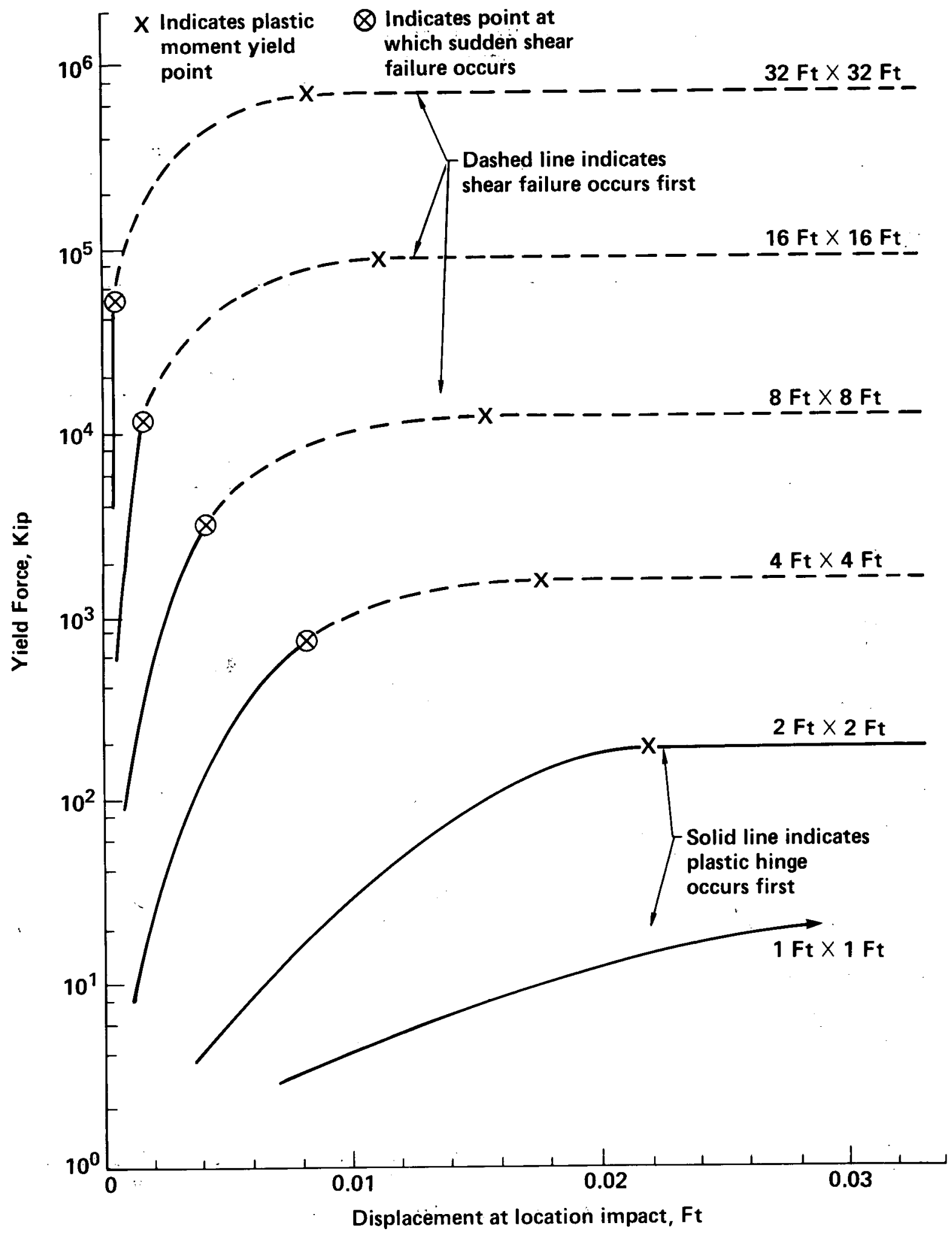

Figure D-6 Force-displacement curves for multi, square bridge columns: 


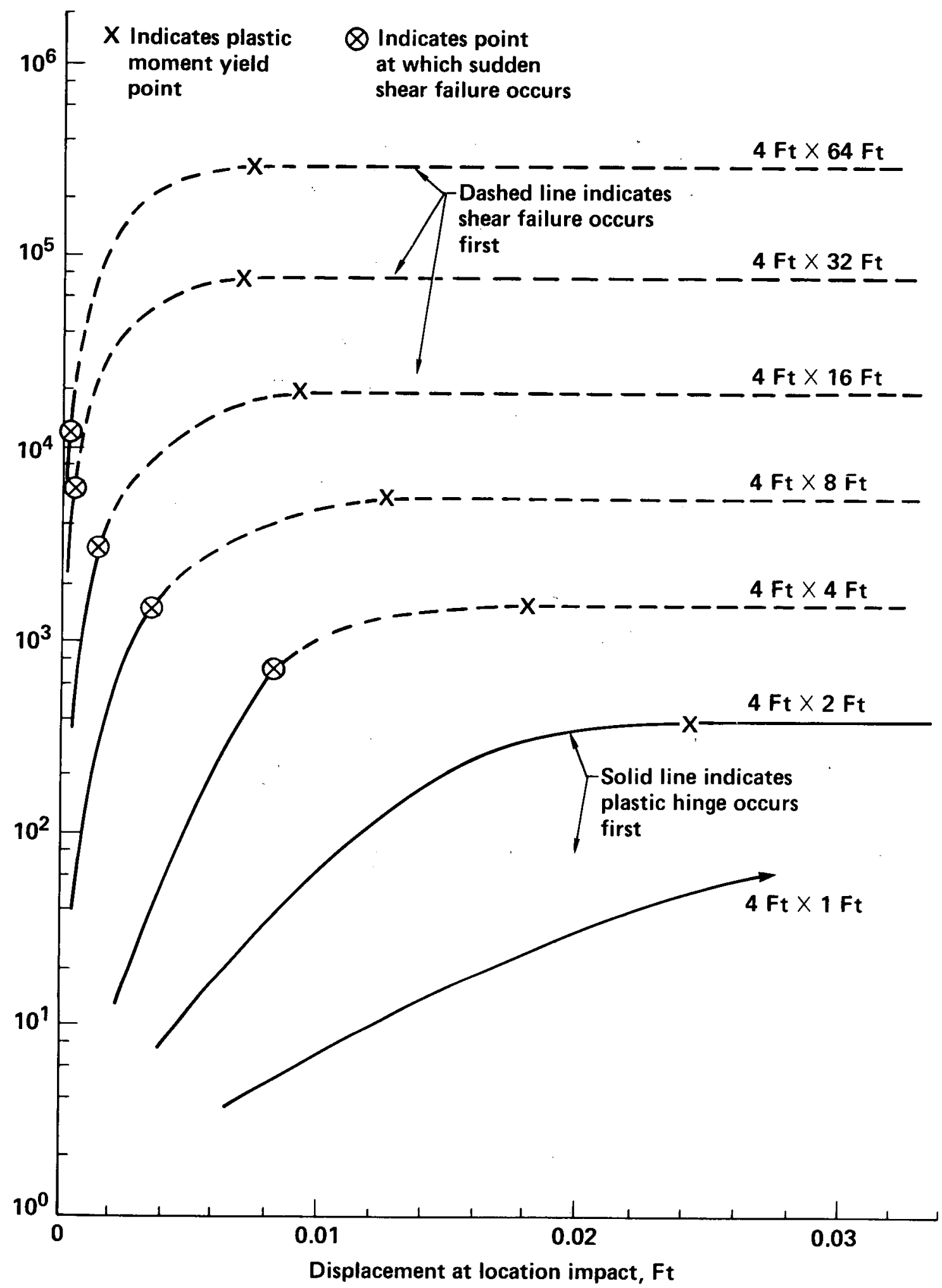

Figure D-7 Force-displacement curves for multi, rectangular bridge columns. 


\section{D.6 References}

D.1 Assessment of the Stiffness Characteristics of Bridge Substructure Components Encountered along a Section of Interstate 5, Engineering Computer Corporation, Sacramento, CA, February 1985. A contractor report to the Lawrence Livermore National Laboratory.

D.2 R. Imbsen, et a1., Soil and Terrain Surveys, Engineering Computer Corporation, Sacramento, CA, January 1985. A contractor report to the Lawrence Livermore National Laboratory.

D.3 Soil Survey, United States Department of Agriculture, Bureau of Chemistry and Soils, Superintendent of Documents, Washington, DC.

D.4 Geological Highway Map, American Association of Petroleum Geologist, TuTsa, OK.

D.5 University of Southern California, Structural Analys is Program 6 (SAP6), University of Southern California, Los Angeles, CA, 1980.

D.6 Engineering Computer Corporation, Reinforced Column (RECOL), Engineering Computer Corporation, Sacramento, CA, 1983. 


\section{APPENDIX E}

List of Figures

Page

E-1 Preliminary truck casks with three types of shielding, used for

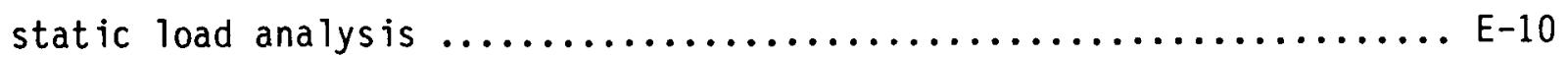

E-2 Preliminary rail casks with three types of shielding, used for

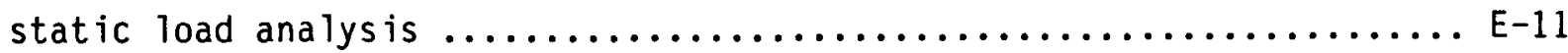

E-3 Representative cask models used for truck and rail cask analysis....... E-13

E-4 Deformation of truck cask during $60 \mathrm{mph}$ impact by a 21 -inch I-beam .... E-15

E-5 Distribution of plastic strain in outer shell due to I-beam impact ....E-16

E-6 Static force versus deflection for endwise loading of truck cask ...... E-18

E-7 Static force versus deflection for sidewise loading of truck cask ..... E-19

E-8 Static force versus deflection for endwise loading of rail cask ...... E-20

E-9 Static force versus deflection for sidewise loading of rail cask ......E-21

E-10 Loading conditions on trees and poles $\ldots \ldots \ldots \ldots \ldots \ldots \ldots \ldots \ldots \ldots . . . \ldots \ldots$

E-11 Finite element mesh for two-element inner-wall model by truck cask..... E-37

E-12 Lead slump in truck cask at $60 \mathrm{mph}$ impact ....................... E-39

E-13 Strain in lower steel structure for truck cask impact at $60 \mathrm{mph} . . .$. . E-40

E-14 Velocity versus time for truck cask impact at $60 \mathrm{mph} \ldots \ldots \ldots \ldots \ldots \ldots$ E-41

E-15 Finite element mesh for rail cask .......................... E-43

E-16 Lead slump in rail cask at $90 \mathrm{mph}$ impact ...................... E-45

E-17 Strain in lower steel structure for rail cask impact at $90 \mathrm{mph} . \ldots \ldots$. E-46

E-18 Velocity versus time for rail cask impact at $90 \mathrm{mph} \ldots \ldots \ldots \ldots \ldots \ldots . . .6-47$

E-19 Model of a truck cask impacting an unyielding surface $\ldots \ldots \ldots \ldots \ldots \ldots$ E-48

E-20 Truck cask impact on unyielding surface at $60 \mathrm{mph} \ldots \ldots \ldots \ldots \ldots \ldots \ldots . . . .60$

E-21 Impact on unyielding surface at $60 \mathrm{mph}$ - maximum plastic strain location ...................................... E-52

E-22 Rail cask impact on unyielding surface at $60 \mathrm{mph} \ldots \ldots \ldots \ldots \ldots \ldots \ldots . . . \ldots$. 53

E-23 Rail cask impact on unyielding surface at $60 \mathrm{mph}$ - maximum plastic strain location ................................. E-55

E-24 Fu11 side drop geometry including impact 1 imiters $\ldots \ldots \ldots \ldots \ldots \ldots \ldots$ E-56 
E-25 Deformations of truck cask during $60 \mathrm{mph}$ side drop (side view) ....... E-58

E-26 Distribution of plastic strain at end of impact (outer shel1) ........E-59

E-27 Comparison of 2-D deformations with 3-D deformations at

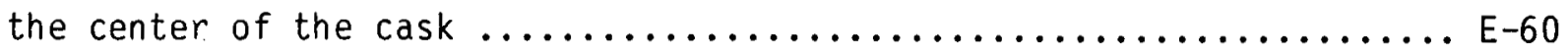

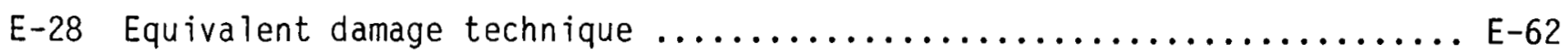

E-29 Soil model comparison with penetration test data $\ldots \ldots \ldots \ldots \ldots \ldots \ldots$ E-75

E-30 Finite element mesh for drops on soils $\ldots \ldots \ldots \ldots \ldots \ldots \ldots \ldots \ldots \ldots . \ldots \ldots$

E-31 Maximum plastic strain location on truck cask for impact

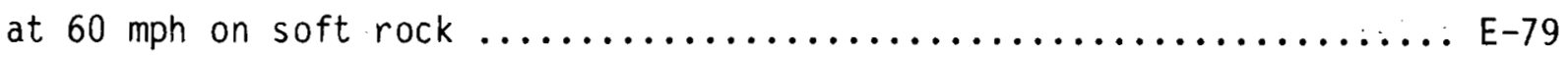

E-32 Maximum plastic strain location on rail cask for impact at

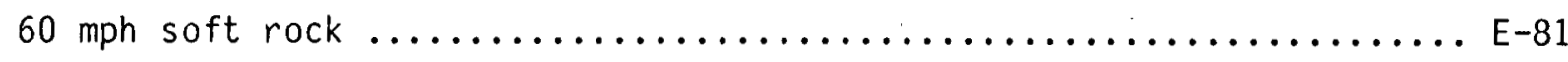

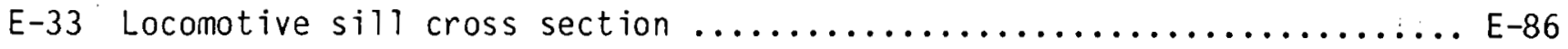

E-34 Sidewise off-center locomotive sill impact $\ldots \ldots \ldots \ldots \ldots \ldots \ldots \ldots \ldots$ E-87

E-35 Thirty mph sidewise off-center sill impact $\ldots \ldots \ldots \ldots \ldots \ldots \ldots \ldots \ldots . . .68$

E-36 Thirty mph sidewise off-center sill impact-maximum plastic strain

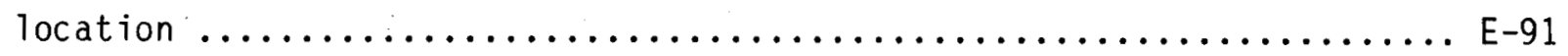

E-37 Model configurations for sidewise head-on sill impact ............ E-92

E-38 Sidewise head-on sili impact at $30 \mathrm{mph} \ldots \ldots \ldots \ldots \ldots \ldots \ldots \ldots \ldots . . . . . . .63$

E-39 Thirty mph sidewise head-on sill impact-maximum plastic

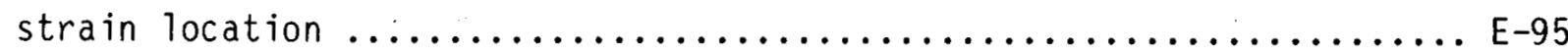




\section{APPENDIX E}

List of Tables

Page

E.1 304 Stainless Steel Structural Properties .................... E-4

E.2 Lead Structural Properties ........................... E-6

E.3 Uranium Structural Properties ......................... E-7

E.4 Balsa Wood Structural Properties ......................... E-9

E.5 Summary of Static Loading Calculations for Six Preliminary

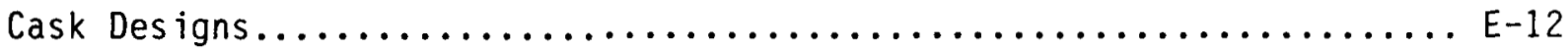

E.6 Bounding Crush Loads Comparison with Crush Loading

Capabilities of the Truck and Rail Casks ................... E-22

E.7 Quasi-Static Force Evaluation for Objects Potentially Impacted ........ E-26

E.8 Impact Velocities Required to Include Cab and Rail Car

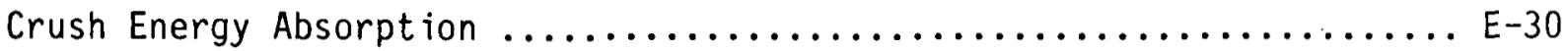

E.9 Truck Cask Strain Response to Impact on Unyielding Surface

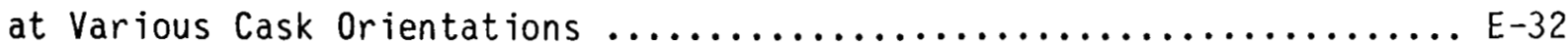

E.10 Rail Cask Strain Response to Impact on Unyielding Surface

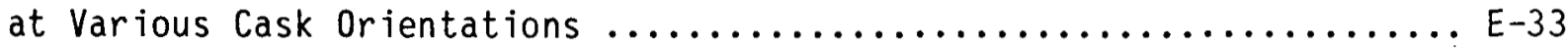

E.11 IMPASC Endwise Impact Benchmark Calculation Against NIKE 2-D ....... E-34

E.12 Summary of Truck Cask Endwise Impact Results .................. E-38

E.13 Summary of Rail Cask Endwise Impact ResuTts ................... E-44

E.14 Results of Truck Cask Sidewise Impact on an Unyielding Surface ....... E-51

E.15 Results of Rai1 Cask Sidewise Impact on an Unyielding Surface ........ E-54

E.16 Comparison of Equivalent Damage Technique Result with Real

Surface Impact Results ............................. E-66

E.17 Soit Parameters ..................................... E-69

E.18 Plate Bearing Test Simulation with NIKE $2-D \ldots \ldots \ldots \ldots \ldots \ldots \ldots \ldots \ldots$ E $\ldots \ldots 1$

E.19 Summary of Soil Types and Range of Soil Parameters ............... E-72

E.20 Selected Soil Parameters for this Study ............................ E-74

E.21 Summary of Cask Responses to Endwise Impacts on Real Surfaces ........ E-77 
E.22 Results of Truck Cask Sidewise Impacts on Real Surfaces

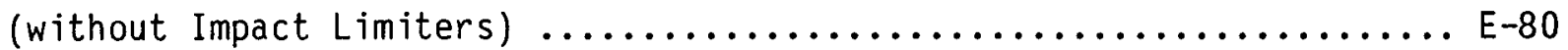

E.23 Results of Rail Cask Sidewise Impacts on Real Surfaces

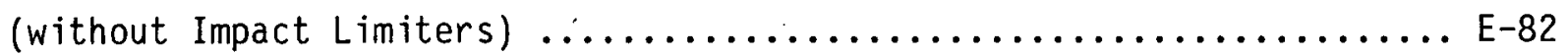

E.24 Interface Force for Water Impact (A11 Results Listed in Multiples

of Cask Weight, No Impact Limiters or Cab Crush Included) ..........E-85

E.25 Results of Sidewise. Off-Center Si11 Impact Aga inst Truck Cask ........ E-90

E.26 Results of Sidewise Head-on Sill Impact Against Truck Cask .......... E-94

E.27 Estimated Response of Rail Cask to Impact by Train Si11 ............ E-97 


\section{APPENDIX E}

Structural Analys is

\section{E.1 Introduction}

This appendix provides the structural models developed and the analyses performed to determine the responses of the representative truck and rail casks to a wide range of impact loads. The family of DYNA and NIKE computer codes were used extensively to calculate the responses of the casks. E.1,E.2

In Section E.2, the material properties used in the process for selecting the representative casks and evaluating the responses of the representative casks are presented. In Section E.3, the static analyses evaluations of different cask designs used to select the representative cask are presented.

In Section E.4, the types of mechanical loading conditions that can affect the strain response of a cask in an accident are discussed. In Section E.5, the quasi-static load evaluation performed for minor accidents are presented. In Section E.6, the structural model and strain response of the two representative casks to impacts on an unyielding surface are discussed. In Section E.7, the response of the two representative casks to impacts on real objects is estimated.

\section{E.2 Materials Properties}

Spent fuel casks must be designed and fabricated to national codes and standards or equivalent requirements. Although there is no specific section in the American Society of Mechanical Engineers (ASME) pressure vessel code applicable to spent fuel casks, the industry has used the ASME code extensively for designing and fabricating spent fuel casks. In this study, to the extent possible, properties of materials were taken from the ASME code. E. 3

Although it is preferred to use probability distributions for material properties that are based on actual fabrication data, discrete bounding values from the ASME code were used in this study. This approach was taken to simplify the modeling and analysis. If distribution had been used, the modeling and analysis would have been unnecessarily complex and unwarranted for the scope of this study. Consequently, conservative material properties 
based on the ASME code were used with loading calculations to estimate seal and weld damage to the representative casks.

Using ASME code properties, limiting plastic strain criteria were used in estimating the response and damage to the representative casks. In this case, the maximum strains would be associated with end-on impact caused by lead slump. Large local strains would be generated at the junctions of the inner containment shell and outer shell with the end closure. Ideally, weld joints would not be present in these areas where high local strains could occur. However, even if welds were present in these areas, most strains would be highly concentrated and could cause only local cracking. Since the extent of lead slump deformation would be limited, it would not be likely that the inner containment would completely rupture. Furthermore, the primary membrane strain on the inner containment cylinder would be compressive and a small fraction of the selected strain levels. The large strains developed at the discontinuities would be highly localized and oriented axially. On the outer she11, the primary membrane strains would be tensile. Even if complete separation from the end plate is postulated, the deformation of the lead would also limit the primary membrane strain to a small fraction of $30 \%$ strain. Consequently, the outer shell would remain intact and continue to maintain the integrity of the lead shielding. In conclusion, the postulated local strains on the order of $30 \%$ would not result in breaching of the cask but may result in local cracking.

Instead of evaluating specific closure and penetration designs, it was assumed for comparative purposes that closure and penetration seals fail when the strain level in the inner shell exceeds $0.2 \%\left(S_{1}\right)$. This approach was based on a review of current cask designs and their ability to withstand impact. forces with large strains. Temperature effects on the material properties were included in the analysis performed. Strain-rate effects were not included for most material properties for the following reasons:

(1) There is no standard set of strain-rate properties in the ASME code or adopted by industry. 
(2) Strain-rate effects generally improve material yield and ultimate strength by $0-30 \%$, but reduce ductility. When strain-rate effects are included for the cask structural materials, then they should be included for surface materials such as rock and concrete. In general, the improvement of material strength properties is greater for ductile type metallic materials than for ceramic type materials. For the purpose of evaluating representative casks impacting representative surfaces, the inclusion of strain-rate effects is not warranted and their exclusion is reasonably conservative.

(3) The strain effect in reducing the structural material ductility was accounted for by using conservative static ultimate strain values for the structural materials.

\section{E.2.1 304 Stainless Steel}

Material properties were obtained for 304 stainless steel from the ASME code.E.3 The properties are tabulated in Table E.1. The elastic-plastic material model used a bilinear fit representation with isotropic hardening. No strain-rate effects were included. The material model used was Material Type 3 in the NIKE 2-D/DYNA 2-D family of finite element codes; the 2-D designation indicating that two-dimensional modeling was performed.E.1,E.2 These codes use an updated geometry to calculate strains. Therefore, it was necessary to use true stress and true strain data, rather than the engineering stress and strain data provided in the ASME code. In order to approximate a value for ultimate true stress, based on ultimate engineering stress, data from Conway, et al., was used.E.4 The stress/strain data of Conway, et al., was not for SA-240, but for another 304 stainless. This, however, provided a means to interpolate a value of true stress for a given engineering stress from the ASME code.

$$
\begin{aligned}
\frac{75,000-71,300}{76,390-71,300} & =\frac{\sigma_{u_{\text {true }}}-85,730}{97,760-85,730} \\
\sigma_{u_{\text {true }}} & =94,475 \text { psi } .
\end{aligned}
$$


Table E.1

304 Stainless Steel Structural Properties

\begin{tabular}{|c|c|c|c|}
\hline Elastic modulus & $E$ & $=$ & $27.6 \times 10^{6}$ ps $i$ \\
\hline Hardening modulus & $E_{n}$ & $=$ & $2 \times 10^{5} \mathrm{psi}$ \\
\hline Poisson's ratio & $v$ & $=$ & 0.29 \\
\hline Engineering ultimate stress & $\sigma_{u}$ & $=$ & $75 \times 10^{3}$ psi \\
\hline True ultimate stress & $\sigma_{u}$ & $=$ & $94.5 \times 10^{3}$ psi \\
\hline Engineering ultimate strain & & $=$ & 0.40 \\
\hline True ultimate strain & & $=$ & 0.34 \\
\hline Yield stress & 0 & $=$ & $25 \times 10^{3}$ psi \\
\hline Density & $\rho$ & $=$ & $7.44 \times 10^{-4} \quad 1 b-\sec ^{2} /$ in $^{4}$ \\
\hline
\end{tabular}


The ultimate engineering strain value provided by the ASME code of $40 \%$ is equivalent to a true strain value:

$$
\begin{aligned}
\varepsilon_{u_{\text {true }}} & =\ln \left(1+\varepsilon_{u_{\text {eng }}}\right)^{\prime} \\
& =\ln (1+0.4) \\
& =0.34 .
\end{aligned}
$$

The ultimate strain percentage used in this study is $30 \%\left(S_{3}\right)$ to accommodate for the effects of strain rate on the reduction of ductility. The hardening modulus was calculated as follows:

$$
E_{n}=\frac{94,475-25,000}{0.34-.00091}=2 \times 10^{5} \text { psi. }
$$

\section{E.2.2 Lead}

The material properties used for lead in this study are presented in Table E.2.E.5 A bilinear fit was used to represent the elastic-plastic material. Strain hardening was used, with isotropic hardening in all calculations. It is considered to be unnecessarily conservative to exclude the strain-rate effect for the lead. The hardening modulus is more significant than the elastic modulus for lead because the lead shield yields relatively easily on impact. The hardening modulus used in this study compares well with the test results reported by Counts and Payne. E.6 Additional benchmarking testing is required to define the lead properties and bonding effects at the cask inner shell with high confidence.

\section{E.2.3 Uranium}

The material properties used for uranium are summarized in Table E.3. E.7 A bilinear fit was used to model its elastic-plastic characteristics for stress/strain. 
Table E.2

Lead Structural Properties

Elastic modulus

$E=2.22 \times 10^{6} \mathrm{ps} i$

Hardening modulus

$E_{n}=4.5 \times 10^{4} \mathrm{psi}$

Poisson's ratio

$v=0.43$

Yijeld stress

$\sigma_{y}=500 \mathrm{psi}$

Density

$\rho=1.06 \times 10^{-3} \mathrm{lb} \mathrm{sec} 2 / \mathrm{in}^{4}$ 
Table E. 3

Uranium Structural Properties

$\begin{array}{lrl}\text { Elastic modulus } & E=26 \times 10^{6} \mathrm{psi} \\ \text { Hardening modulus } & E_{n}=1 \times 10^{6} \mathrm{psi} \\ \text { Poisson's ratio } & \nu=0.21 \\ \text { Yield stress } & \sigma_{y}=4.6 \times 10^{4} \mathrm{psi} \\ \text { Density } & \rho=1.74 \times 10^{-3} \mathrm{lb}-\mathrm{sec}^{2} / \mathrm{in}^{4}\end{array}$




\section{E.2.4 Balsa Wood}

An elastic-plastic model was selected for modeling the balsa wood. E.8 The material properties used are tabulated in Table E.4.E.8 Material Type 10, from DYNA 2-D, was used for the wood material model.

\section{E.3 Preliminary Cask Designs and Cask Selection}

Six preliminary cask designs were developed to perform screening analyses to assess their responses to high-loading conditions. The designs included the use of three types of gamma shielding materials: lead, depleted uranium, and steel. Three truck and three rail casks were developed using each type of shielding. The pertinent materials and dimensions for the six preliminary cask designs are provided in Figs. E-1 and E-2 for the truck and rail casks, respectively.

Static force evaluations were performed using the NIKE 2-D finite element computer code for the six casks. The loading conditions applied on each of the casks are illustrated in Table E.5. In case (a), a pressure load was applied on one end over the entire closure region of the cask in increments of 200 psi with the other end of the cask resting on an unyielding surface. In case (b), a circular cross-section of the cask was loaded in increments of 200 psi over the top area of the cask with the bottom resting on an unyielding surface. In case (b), the model had a unit or one inch thickness. The yield force results of the two loading calculations for each of the six casks are summarized in Table E.5. The lead cask yielded at significantly lower loading conditions in all cases. Based on these results, the lead shielded cask was selected for developing a representative cask design for impact analysis.

\section{E.4 Mechanical Loading Conditions Caused by Accidents}

Mechanical loading conditions on a cask caused by an accident can result in damage to the inner shell of the cask. Mechanical loading conditions include impact, puncture (including missiles), and crush. Two representative cask designs were developed as shown in Fig. E-3: one for truck shipments and one for rail shipments of spent fuel. The representative truck cask 
Table E.4

Balsa Wood Structural Properties

Elastic modulus

Poisson's ratio

Yield stress

Sheer modulus

Density

$$
\begin{aligned}
E & =5.9 \times 10^{5} \mathrm{psi} \\
\nu & =0.0 \\
\sigma_{y} & =1.7 \times 10^{3} \mathrm{psi} \\
G & =2.95 \times 10^{5} \mathrm{psi} \\
\rho & =1.35 \times 10^{-5} \mathrm{pb}-\mathrm{sec}^{2} / \mathrm{in}^{4}
\end{aligned}
$$

E-9 

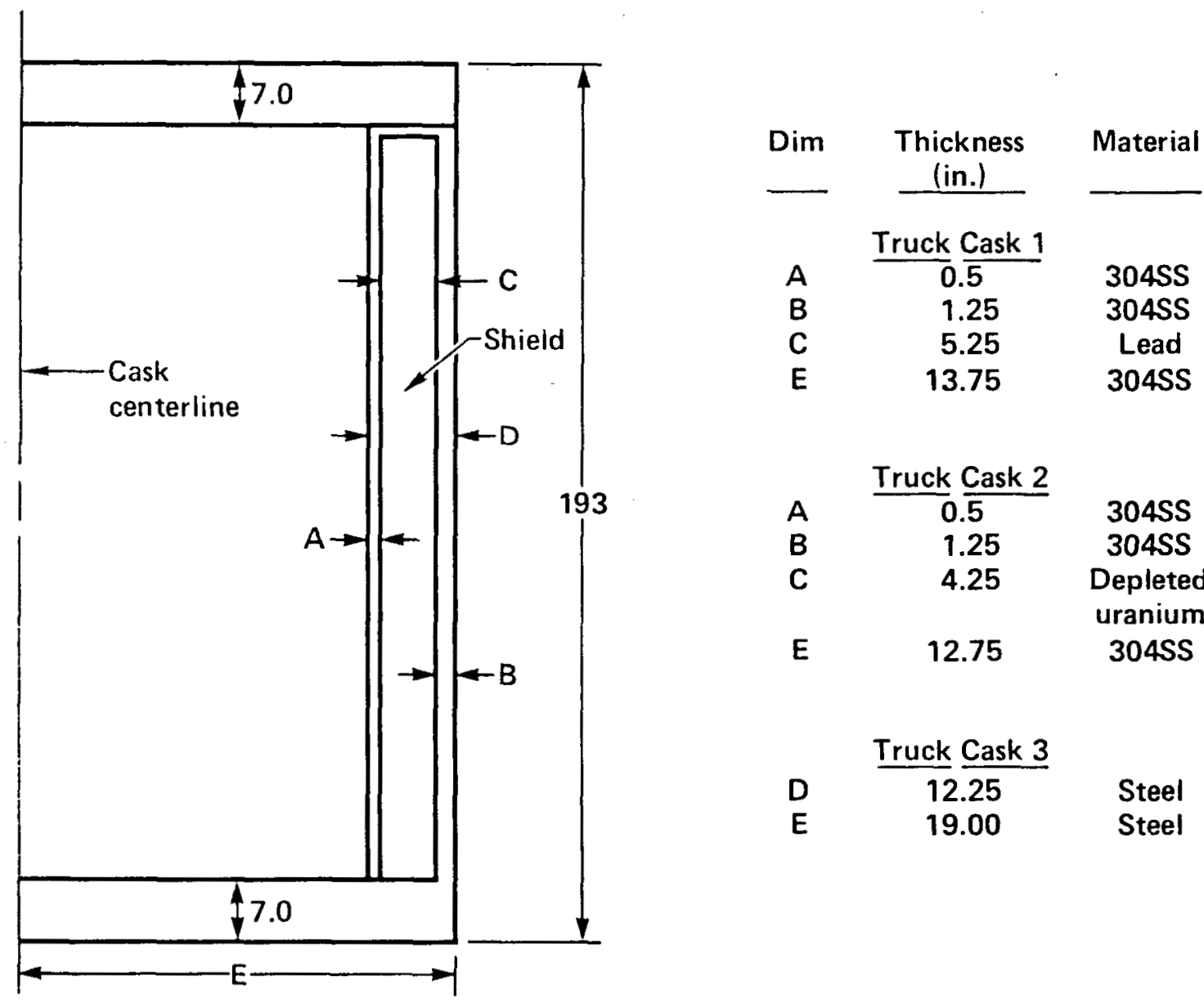

\begin{tabular}{|c|c|c|}
\hline \multicolumn{3}{|c|}{ Truck Cask 2} \\
\hline A & $0 . \overline{5}$ & $304 S S$ \\
\hline B & 1.25 & 304SS \\
\hline C & 4.25 & $\begin{array}{l}\text { Depleted } \\
\text { uranium }\end{array}$ \\
\hline $\mathbf{E}$ & 12.75 & 304SS \\
\hline
\end{tabular}

Truck Cask 3

$\begin{array}{lll}\text { D } & 12.25 & \text { Steel } \\ \text { E } & 19.00 & \text { Steel }\end{array}$

Figure E-1 Preliminary truck casks with three types of shielding, used for static load analysis.

$$
E-10
$$




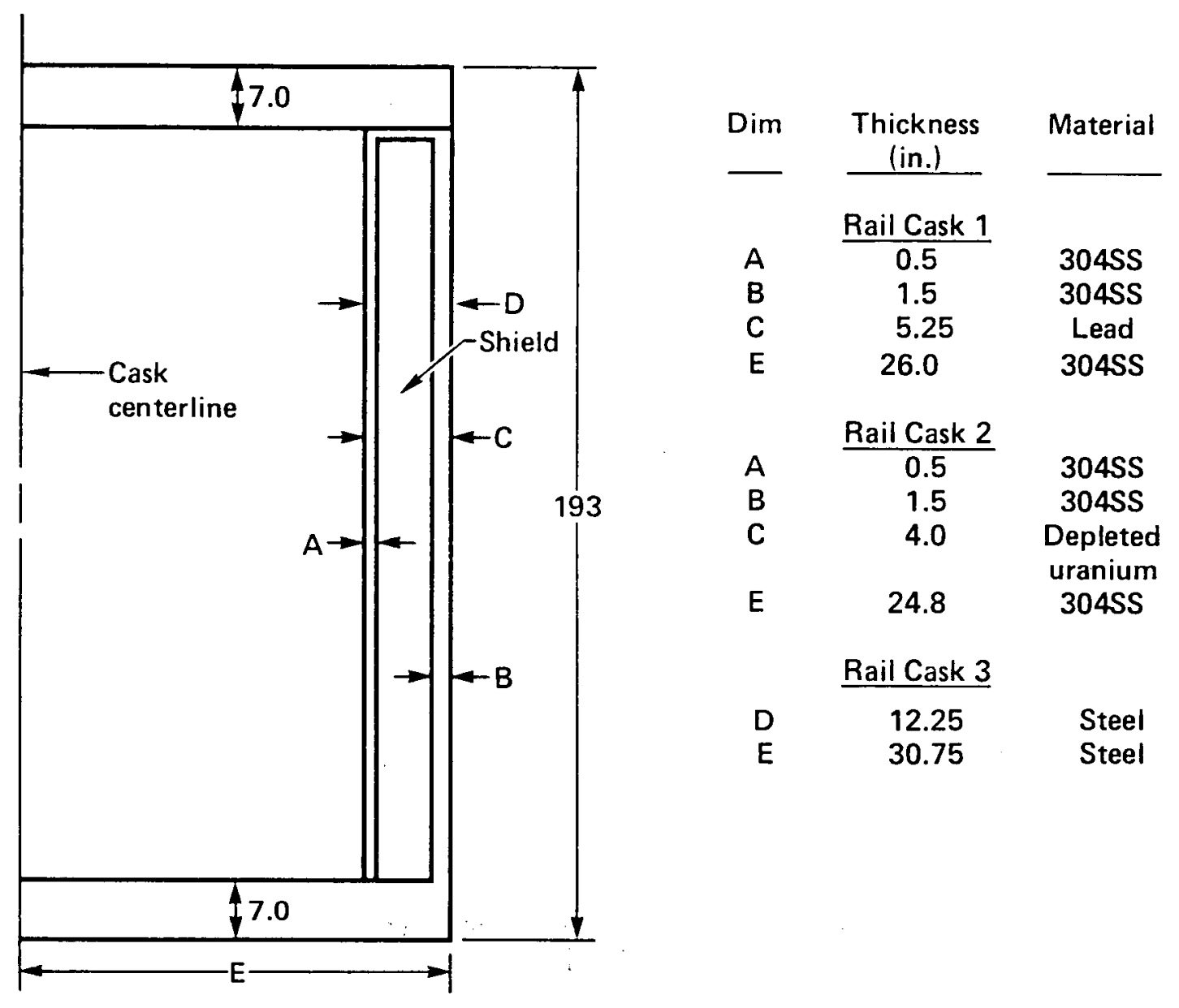

Figure E-2 Preliminary rail casks with three types of shielding, used for static load analysis. 
Table E.5

Summary of Static Loading Calculations for Six Preliminary Cask Designs

Loading

Configuration

Case (a), endwise

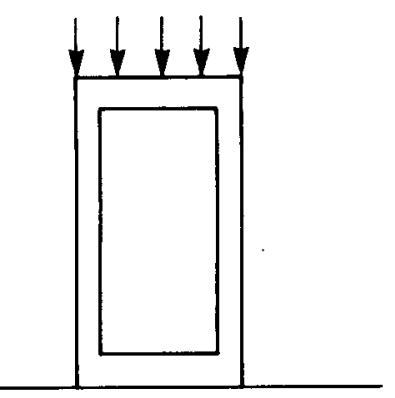

Case (b), sidewise

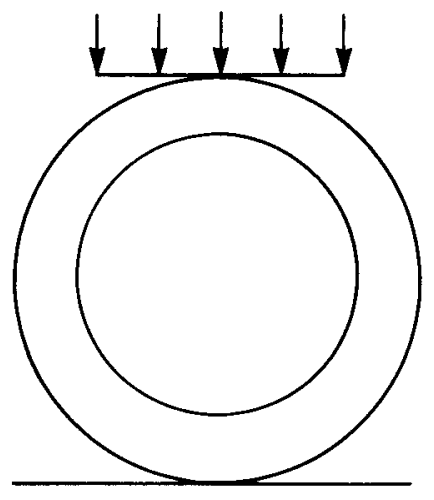

Cask

Type

Yield

Force (1bs)

ENDWISE LOADING

Truck

Lead $\quad 3,300,000$

Depleted uranium $8,000,000$

Stee 1

$11,000,000$

Rail

Lead

Depleted uranium

Stee 1

$8,000,000$

$17,000,000$

$40,000,000$

SIDEWISE LOADING

Truck

Lead

Depleted uranium $11,000,000$

Stee 7

$26,000,000$

Rail

Lead

Depleted uranium $3,700,000$

Stee 1 

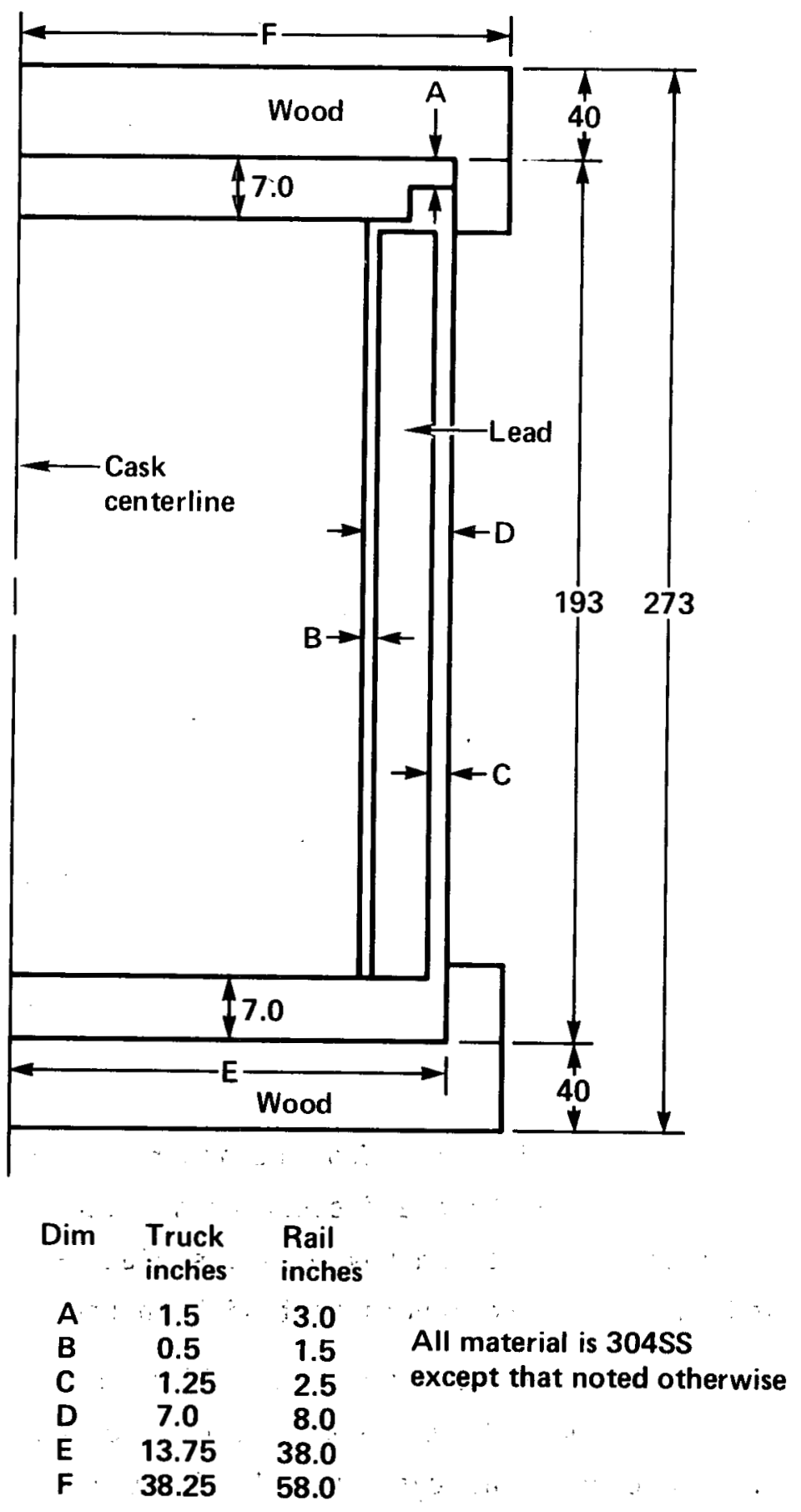

Figure E-3 Representative cask models used for truck and rail cask analysis.

$$
E-13
$$


(Fig. E-3) design uses the same dimensions as the preliminary lead truck cask design (Fig. E-1). The truck cask design allows transport of a single PWR fuel assembly. The representative rail cask design (Fig. E-3) dimensions differ from the preliminary lead rail cask design (Fig. E-2). The capacity of the rail cask is 21 PWR fuel assemblies which reflects the greater capacities of anticipated cask designs. Each design uses helium in the cask cavity.

Typically, as discussed in Sections E.6 and E.7, the dynamic force caused by impact on a hard surface can be in the range of 1-10 million pounds on the representative truck cask depending on the impact velocity (velocity component perpendicular to the surface impacted), the cask orientation, and the hardness of the surface. The strain at the inner wall of the cask can exceed $30 \%\left(S_{3}\right)$ at impact velocities greater than $75 \mathrm{mph}$. The dynamic forces generated by impacts on a hard surface are even higher for the rail cask compared to the truck cask because of the larger size and weight of the rail cask.

The possibility of puncture of the cask by a high energy-density object was evaluated. It was concluded that a high velocity I-beam would have the highest energy density of probable missiles generated in an accident and that the I-beam represented the bounding case for the puncture of a cask wall. E.9 Assuming that the I-beam is the bounding case, the representative truck cask was analyzed with DYNA 3-D (the 3-D designation indicating that threedimensional modeling was performed) for impact by a high energy I-beam.

The representative truck cask and I-beam were modeled using two planes of symmetry. The truck cask model included the inner and outer steel walls and the lead shielding but did not have end closures or impact limiters. The back side of the cask was supported by an unyielding surface. The 40 foot WF-21/96 I-beam was modeled as $1 / 4$ of the length unit with an equivalent weight.

The impact velocity was $60 \mathrm{mph}$, resulting in an impact force of 40,000 pounds by the I-beam. The deformations due to the impact are shown in Fig. E-4. The impact caused the cask wall to flatten locally and the I-beam to yield significantly at the point of impact. A maximum plastic strain of $5 \%$ developed in the outer wall of the cask as shown in Fig. E-5. The maximum 

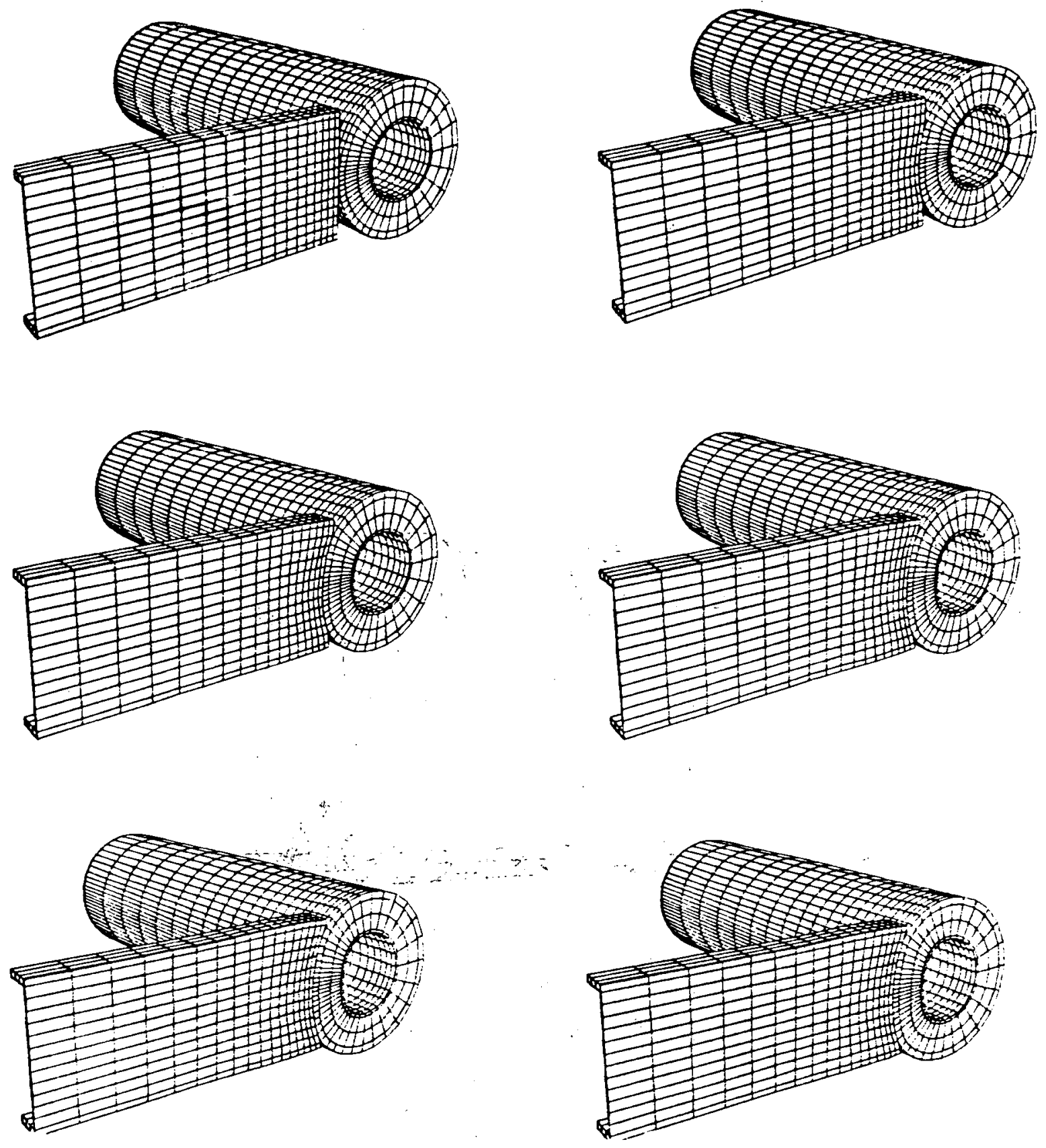

Figure E-4 Deformations of truck cask during $60 \mathrm{mph}$ impact by a 21-inch I-beam. 
TIME $=6.00102 E-03$

CONTOURS OF EFF. PLASTIC STRAIN

$M I N=0$.

$M A X=4.940 E-02$ IN ELEMENT 921
CONTOUR VALUES

$A=0$.

$B=6.00 E-03$

$C=1.20 E-02$

$D=1.80 E-02$

$\mathrm{E}=2.40 \mathrm{E}-02$

$F=3.00 E-02$

$\mathrm{G}=3.60 \mathrm{E}-02$

$\mathrm{H}=4.20 \mathrm{E}-\mathrm{O} 2$

$I=4.80 \mathrm{E}-02$

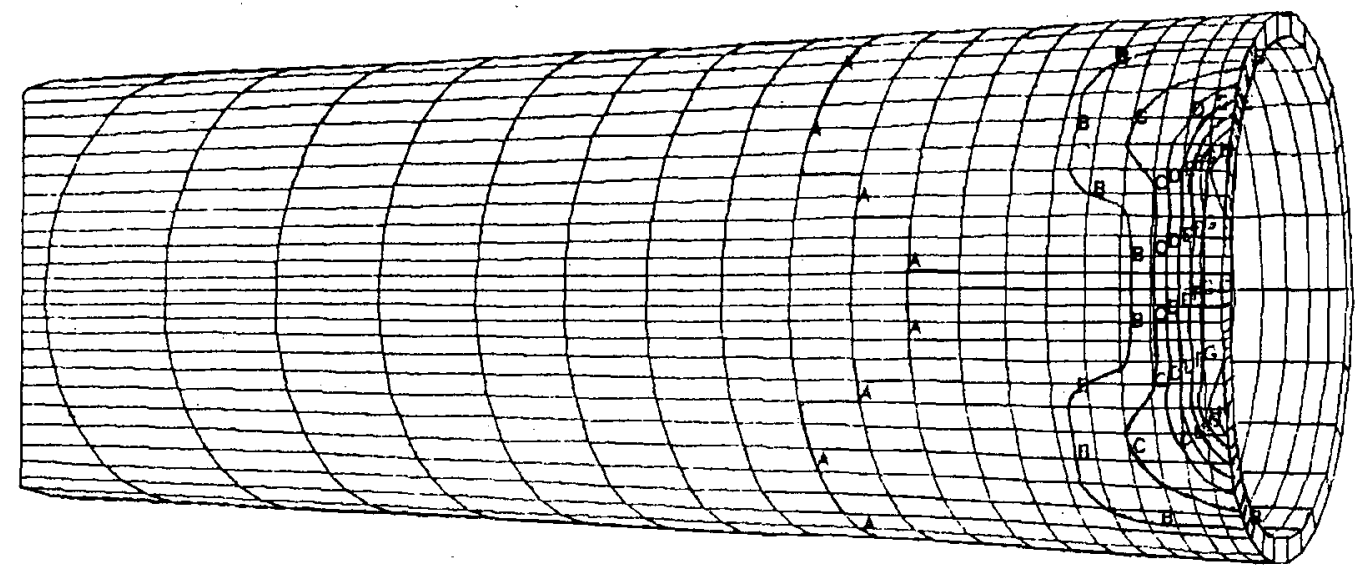

Figure E-5 Distribution of plastic strain in outer shell due to I-beam impact. 
stress and shear in the outer wall were 34,950 psi and 19,500 psi, respectively. The I-beam did not penetrate the cask wall.

In comparison with the I-beam impact, the train sill impact discussed in Section 7.4 on the truck cask is more severe. The impact force exceeded 9 million pounds and the strain was $20 \%$ for a $60 \mathrm{mph}$ impact. Therefore, it was concluded that the impact by a train sill is a more severe accident that may cause high local strains and stress to the cask walls. Due to the larger size and weight of the rail cask, it was also concluded that the impact of a train sill on the rail cask is more severe than the impact by an I-beam.

The possibility of crush of the representative casks by a heavy object was evaluated. Static force evaluations of the representative casks shown in Fig. E-3 were performed using the NIKE 2-D finite element computer code. As discussed in Section E.3, the loading conditions applied on each of the representative casks are the same as those used for the preliminary cask designs in Table E.5. The force deflection characteristics for each of the representative casks are shown in Figs. E-6 through E-9. The force where general yielding of the cask body occurs was selected for comparing their loading capabilities with the bounding crush loads from NUREG/CR-3498. E.9

In Table E.6, typical crush loads that could occur in real accidents are compared with the crush loading capabilities of the representative casks. The bounding crush load is a 200-ton locomotive that would rest on the rail cask by its sill. Both the truck and rail cask can support the weight of the locomotive without yielding.

Based on severe accident data, the frequency of occurrence of impact loads is at least a factor of 10 times higher than for puncture or crush loads. Therefore, since impact can generate higher loads and can occur more frequently, it is concluded that impact loads dominate the potential mechanical loading environment and only impact loads will be considered further. 


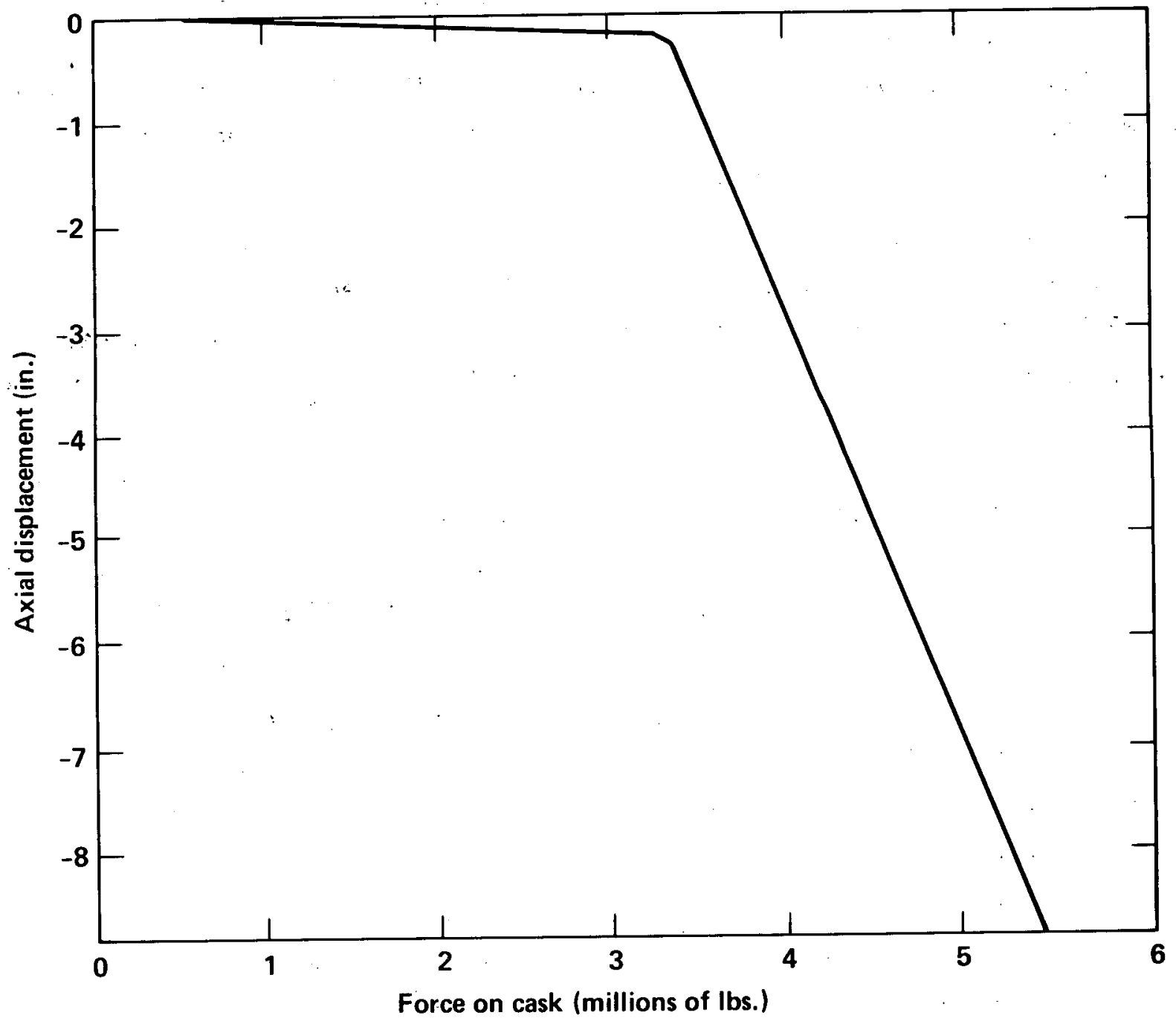

Figure E-6 Static force versus deflection for endwise loading of truck cask. E-18 


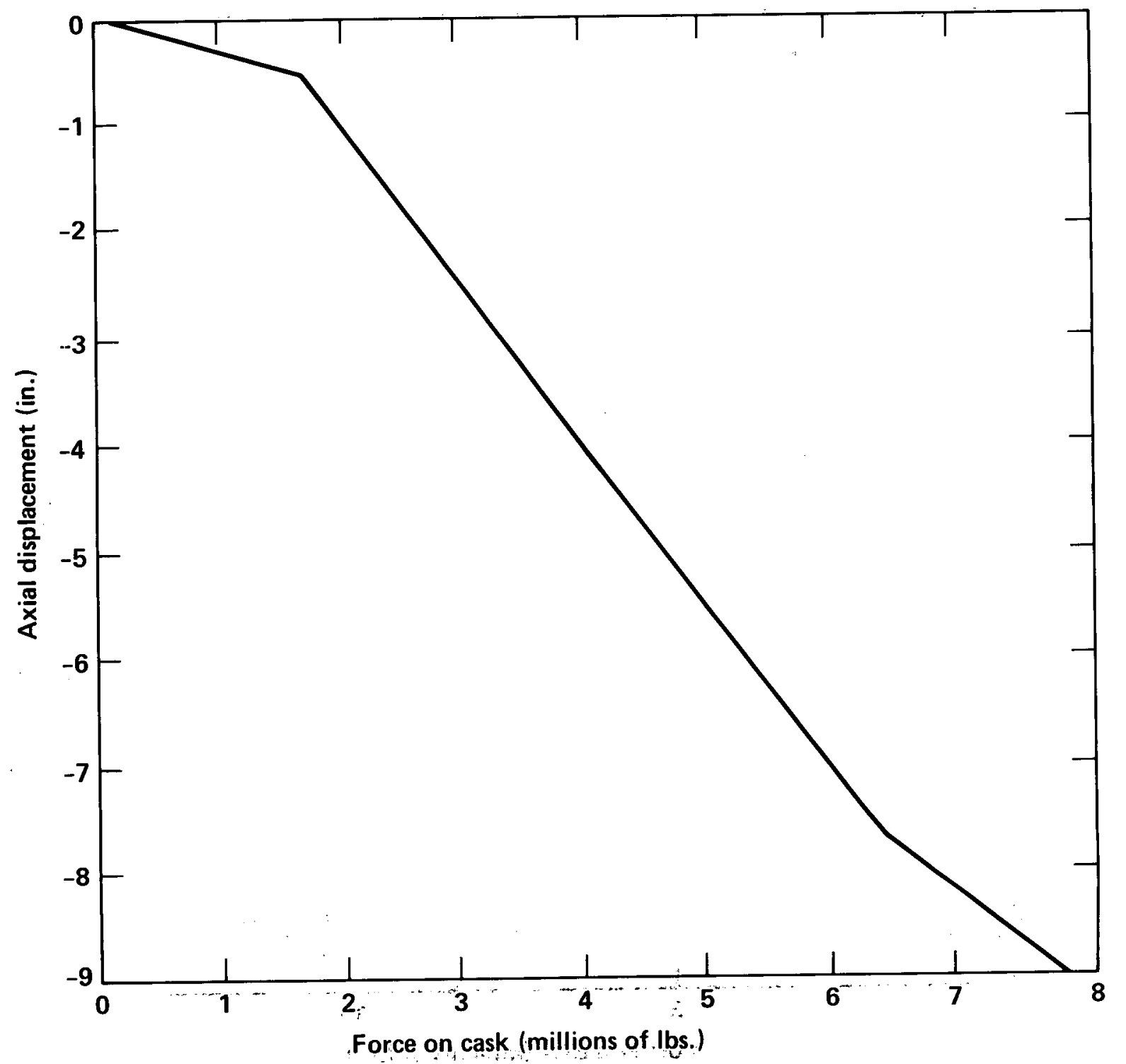

Figure E-7 Static force vërsus deflection for sidewise loading of truck cask.

$$
\text { E-19 }
$$




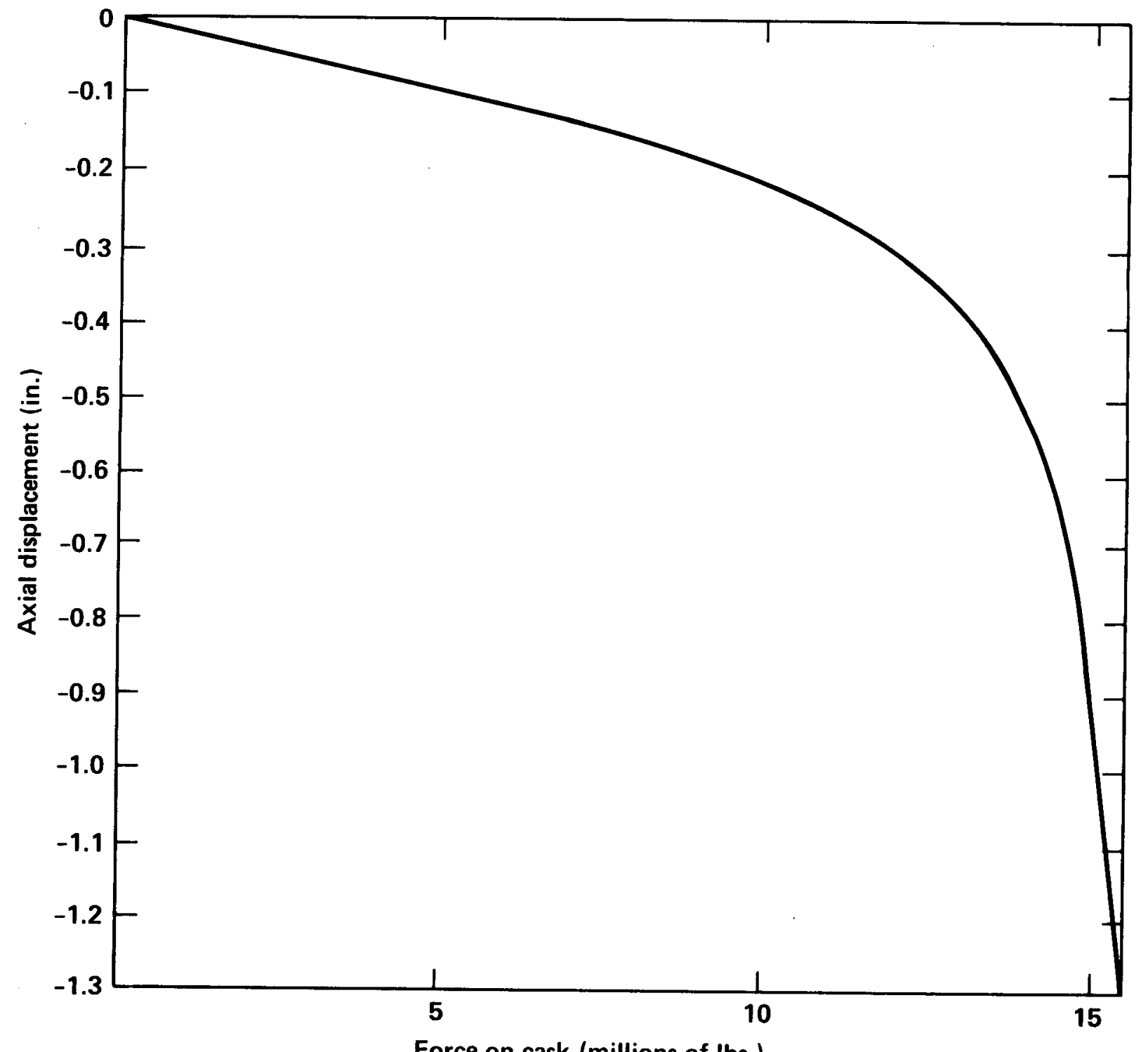

Force on cask (millions of lbs.)

Figure E-8 Static force versus deflection for endwise loading of rail cask. 


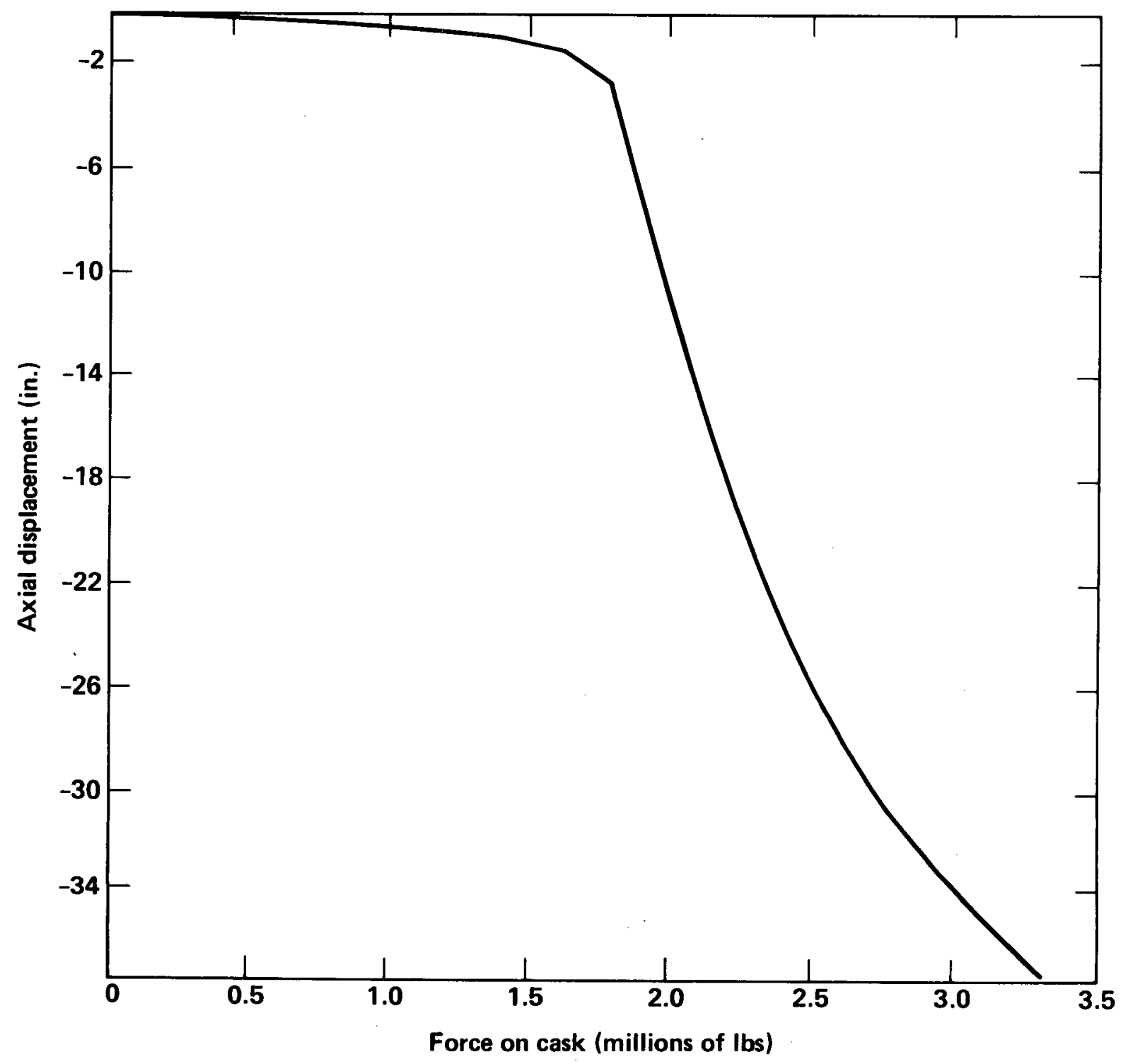

Figure E-9 Static force versus deflection for sidewise loading of rail cask. 
Table E.6

Bounding Crush Loads Comparison with Crush Loading

Capabilities of the Truck and Rail Casks

\begin{tabular}{|c|c|c|c|}
\hline $\begin{array}{l}\text { Bounding Crush } \\
\text { Force Description }\end{array}$ & $\begin{array}{l}\text { Resultant Force } \\
\text { (lbs) }\end{array}$ & $\begin{array}{c}\text { Truck Cask } \\
\text { Capabil ity } \\
\text { (1bs) }\end{array}$ & $\begin{array}{c}\text { Rail Cask } \\
\text { Capability } \\
\text { (1bs) }\end{array}$ \\
\hline $\begin{array}{l}\text { For highway accidents } \\
\text { the weight of a } 60,000 \\
\text { pound truck with its } \\
\text { contents. Weight is } \\
\text { carried across truck } \\
\text { frame width. }\end{array}$ & 60 thousand & 1.6 million & 1.6 million \\
\hline $\begin{array}{l}\text { For railway accidents } \\
\text { the weight of a } 200 \\
\text { ton locomotive. Weight } \\
\text { is distributed across } \\
\text { the train sill. }\end{array}$ & 400 thousand & 1.6 million & $1.6 \mathrm{million}$ \\
\hline
\end{tabular}




\section{E.5 Quasi-Static Loads Due to Minor Accidents}

In Section E.4, the minimum static force required to yield either the representative truck or rail cask was determined to be 1.6 million pounds. The static force required to yield the impacted object completely is in most cases significantly less than 1.6 million pounds. The static force required to yield either the representative truck or rail cask was compared with the force required to collapse potential objects to screen out low resistance objects from further analysis.

The maximum force that an object can generate during a high velocity impact was estimated using quasi-static methods. D'Alembert's principle was used to establish static force equivalent to the inertial force caused by deceleration. It was concluded that objects such as automobiles or truck trailers cannot generate forces greater than 400,000 pounds even at high velocities.

The static force required to collapse an automobile is less than 50,000 pounds. E. 10 The maximum impact forces for rail cars and truck tractortrailers are estimated from the static forces reported for the crash tests of spent fuel casks.E.11,E.12 The quasi-static forces for concrete structures such as walls, columns, : and abutments were estimated from the mechanical loading analyses of the roadside structures given in Appendix $D$.

The method used to determine the maximum impact force trees and posts could resist was a one-dimensional (1-D) beam hand, calculation to determine the limit moment. The loading condition we assumed is shown on Fig. E-10a. A plastic hinge forms when the entire tree/pole cross section yields at the location of maximum moment as shown in Fig. E-10b. - A yield stress of $8,400 \mathrm{psi}$ is assumed, based on three times the allowable for Douglas fir. E.13 The bounding force (force to produce plastic hinge) for a solid circular Douglas fir cross section is $P=233.38 R^{3}$ ibs, where $R$ is the radius of the tree in inches.

The bounding force for a pole, assuming a yield strength of $36 \mathrm{ksi}$, is

$$
P=1000 R_{0}\left(R_{0}^{2}-R_{i}^{2}\right)\left(\frac{1}{2-\frac{t}{R_{0}}}+1-\frac{t}{R_{0}}\right)
$$


(a)

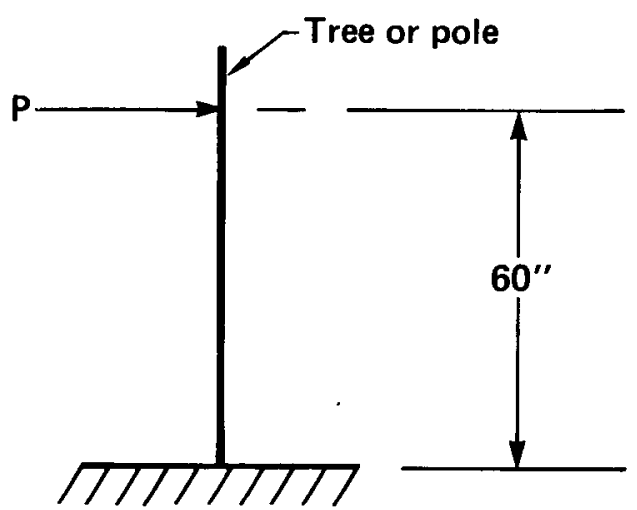

(b)

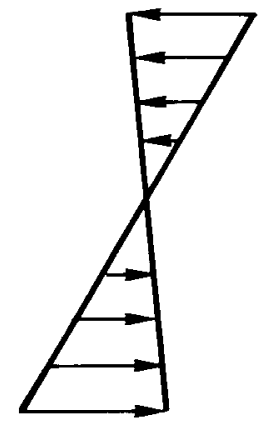

elastic

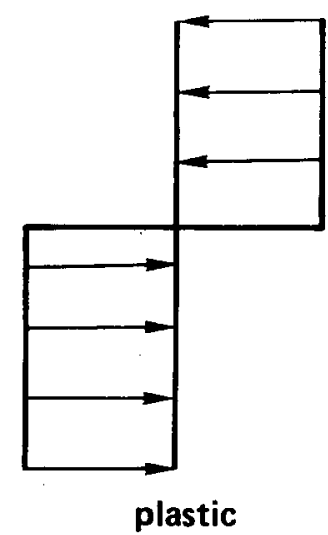

plastic

Plastic hinge forms when the entire cross section yields

Figure E-10 Loading conditions on trees and poles. 
where

$$
\begin{aligned}
& R_{0}=\text { the pole outside diameter, inches, } \\
& R_{i}=\text { the pole inside diameter, inches, } \\
& t=\text { the pole wall thickness, inches. }
\end{aligned}
$$

Two examples of minor target bounding forces follow: a 1.5-foot-diameter Douglas fir tree has a bounding force of $1.7 \times 10^{5} \mathrm{lbs}$, and a 10-inch-diameter standard schedule pole has a bounding force of $2.95 \times 10^{4}$ lbs.

Low-resistance objects such as trees, road signs, electricity poles, motorcycles, passenger cars, trailers, and trucks can be screened out based on static analysis. Assuming that the impact force is linearly applied, the force/unit length that could cause local deformation can be estimated. The representative cask can resist a linear force of 100,000 pounds/foot to generate a strain of less than $0.2 \%\left(S_{1}\right)$ at the inner shell. The linear force to crush objects in many accidents is much less than 100,000 pounds/foot. Table E.7 lists objects that are typically impacted in an accident, many of which do not generate a maximum total force greater than 400,000 pounds or a linear force greater than 100,000 pounds/foot.

Stronger and more massive objects, such as trains, bridge columns, abutments, and real surfaces such as roadbeds are analyzed in Section E. 7 .

\section{E.6 Impacts on Unyielding Surfaces}

Impact calculation for the representative casks onto unyielding surfaces were divided into two categories: those where the cask structural response is essentially elastic and those where the cask structural response is elasticplastic. The elastic response evaluations discussed in Subsection E.6.1 were performed primarily using the 1-D beam element code IMPASC. ${ }^{2} 14$ The elasticplastic response evaluations discussed in Subsection E.6.2 were performed using the DYNA and NIKE family of computer codes. 
Table E.7

Quasi-Static Force Evaluation for Objects Potentially Impacted

\begin{tabular}{lrr}
\hline Object & $\begin{array}{c}\text { Total Force } \\
(1 \mathrm{bs})\end{array}$ & $\begin{array}{c}\text { Linear Force } \\
(1 \mathrm{bs} / \mathrm{ft})\end{array}$ \\
\hline Truck Cask & & \\
Endwise & $3,300,000$ & 100,000 \\
Sidewise & $1,600,000$ & \\
Rail Cask & & 100,000 \\
Endwise & $13,000,000$ & $<10,000$ \\
Auto Sidewise & $1,600,000$ & $<17,000$ \\
Truck Tractor & 50,000 & $<70,000$ \\
Truck Trailer & 100,000 & $>250,000$ \\
Train & 450,000 & $<10,000$ \\
Motorcycle & $2,000,000$ & $<50,000$ \\
Bus & 20,000 & $<50,000$ \\
Sound Wal1 & 300,000 & $>225,000$ \\
$4 \times 4$ Column & 50,000 & \\
\hline
\end{tabular}




\section{E.6.1 Elastic Response of the Cask}

In order to perform the response calculation, it is essential that a proper computer code be selected. This computer code(s) must have the following special capacities or features:

1. Can provide dynamic impact analys is

2. Can analyze oblique impact

3. Can analyze impact : limiter non linear behavior

4. Can analyze lead slump effect

5. Can be run inexpensively.

Three computer codes were selected, NIKE 2-D/3-D, E.1 DYNA 2-D/3-D, E.2 and IMPASC. E.13 Each code has its special features, but also has weaknesses in meeting all the requirements. NIKE 2-D/3-D and DYNA 2-D/3-D are two of the most powerful finite element codes for dynamic impact analysis. They meet a 1 the requirements listed above except that they are expensive to run. Especially when dealing with oblique impact and nonlinear impact limiters, the required 3-D modeling can result in costly calculations.

In order to manage the large amount of tanalys is required for this study, a code had to be found that could do analys is less expensively. The IMPASC code was selected. IMPASC was developed specifically for dynamic impact analys is of shipping casks to assess whether they meet the 10 CFR 71 design requirements. It has a special feature for handling oblique impact. This code can also analyze nonlinear behavior of the impact limiter, and is inexpensive to run. The deficiency is that IMPASC cannot assess the leadslump effect.

The approach benchmarked the IMPASG results with DYNA/NIKE results to assess the lead slump, and then used the IMPASC code to run production calculations for impacts on an unyielding surface. From the sensitivity study 
performed with the DYNA/NIKE codes in Subsection E.6.1.3, it was found that lead slump will not occur under any conditions as long as the axial force on the cask is smaller than $40 \mathrm{~g}$. This is also the level at which it could be assured that the strain on the inner shell is less than $0.2 \%\left(S_{1}\right)$ and the closure seal is functional, since the impact limiter is designed to completely absorb the energy of this impact force level.

Sensitivity studies were performed to show that the inclusion of the cask contents does not significantly change the strain levels in the cask. The sensitivity studies included the following: lumping the weight of the contents at the bottom end of the cask, modeling the contents as elements with mass but no stiffness in the cask cavity, and modeling the contents with mass and an estimated stiffness to simulate fuel bundles and the fuel basket. Liquids such as water are not contained in the cask, because helium is the coolant. The resulting changes in stress-strains and g loads for the various models were not significant for the purposes of this study.

\section{E.6.1.1 Truck Cask Impact}

The IMPASC code was used to perform impact analys is on an unyielding surface for the truck cask. The analysis was done by varying the other two parameters: cask orientation angle and impact velocity. The cask response was calculated for the cask orientation angles of $0^{\circ}, 10^{\circ}, 30^{\circ}, 50^{\circ}, 70^{\circ}$, and $90^{\circ}$ and impact velocities of $30 \mathrm{mph}, 38 \mathrm{mph}$, and $45 \mathrm{mph}$. The impact velocity is defined as the velocity component in the direction perpendicular to the impact surface. The $0^{\circ}$ cask orientation angle represents impact to the side of the cask, whereas the $90^{\circ}$ cask orientation angle represents impact to the end of the cask.

For the $90^{\circ}$ angle case, the effects due to the truck cab crushing and lead slump pressure were included. The effects of lead pressure were calculated using NIKE and are discussed in Subsection E.6.1.3. The effects of the cab crush for front-wise impacts, which can be taken into account by increasing the impact velocity required to give equivalent strain, was estimated using an energy balance. The energy absorbed by the cab is estimated as 


$$
E_{a}=F_{I} \times d
$$

where $F_{I}$ is the impact or crush force of the truck $c a b$ in inches and $d$ is the total distance the cab can be crushed in inches. E.11 The kinetic energy required to cause the same response for the cask when the cab crush is included is estimated as

$$
\left(\frac{1}{2} M V_{2}^{2}\right)_{C}=E_{a}+\left(\frac{1}{2} M V_{1}^{2}\right)_{W C}
$$

where $M$ is the mass of the truck and cask in 1bs; $V_{2}$ is the impact velocity in $\mathrm{ft} / \mathrm{sec}$ used to find the strain, taking into account $\mathrm{cab}$ crush energy absorption; and $V_{1}$ is the impact velocity in $\mathrm{ft} / \mathrm{sec}$ without cab crush energy absorption as used in IMPASC code calculations. The mass of the truck was taken from SAND77-0270. E.11 The velocity required to cause the same cask response when cab crush is considered is

$$
v_{2}^{2}=-\frac{2 E_{a}+M V_{1}^{2}}{M}
$$

The effects of cab crush are included only for impact velocities up to $60 \mathrm{mph}$; at higher velocities the cask will break from its tie-downs and leave the truck without any velocity reduction caused by truck cab crush. E.11 Table E.8 summarizes the velocities required to cause the same cask response when cab crush is included as compared to the velocities without cab crush. The effective impact velocity to take into account cab crush, $v_{2}$, is used to determine the strain for a given impact velocity as calculated by the IMPASC code. For instance, the strain at $30 \mathrm{mph}$ as calculated by IMPASC for a truck cask is assumed to occur at $34.6 \mathrm{mph}$ when cab crush is taken into account. 
Table E.8

Impact Velocities Required to Include Cab and

Rail Car Crush Energy Absorption

\begin{tabular}{ccc}
$\begin{array}{c}\text { Velocity without } \\
\text { Crush } \\
\text { (mph) }\end{array}$ & $\begin{array}{c}\text { Velocity with } \\
\text { Cab Crush } \\
\text { (mph) }\end{array}$ & $\begin{array}{c}\text { Velocity with } \\
\text { Rail Car Crush } \\
\text { (mph) }\end{array}$ \\
\hline 30 & 34.6 & 35 \\
45 & 48.2 & 48.5 \\
60 & 62.4 & 62.8 \\
\hline
\end{tabular}


The strain variation with cask orientation angle for various impact velocities are given in Table E.9. From these results it was concluded that for the representative truck cask the endwise and sidewise strain responses bound the strain responses for all cask orientations. For cask orientations from $0-90^{\circ}$ the structural strain responses can be linearly interpolated between the sidewise and endwise strain responses. The strain is $0.2 \%\left(S_{1}\right)$ at the impact velocity of $32 \mathrm{mph}$ for sidewise impacts and $38 \mathrm{mph}$ for endwise impacts.

\section{E.6.1.2 Rai1 Cask Impact}

The IMPASC code was used to perform these analyses. The analys is was done by varying the other two parameters, i.e., cask orientation angle and impact velocity. The cask response was calculated for the cask orientation angles of $0^{\circ}, 10^{\circ}, 30^{\circ}, 50^{\circ}, 70^{\circ}$, and $90^{\circ}$ and impact velocities of $30 \mathrm{mph}, 45$ $\mathrm{mph}$, and $60 \mathrm{mph}$. The impact velocity is defined as the velocity component in the direction perpendicular to the object surface. The $0^{0}$ cask orientation angle represents the impact to the side of the cask, whereas the $90^{\circ}$ cask orientation angle is the impact to the end of the cask. The results of this sensitivity study are given in Table E.10. As done for the truck cask, for the $90^{\circ}$ angle case we included the effects of lead slump pressure and crushing the front end of the rail car transporting the cask. Table E.8 summarizes the velocities required to include the rail car crush effects. From the results it is concluded that for the representative rail cask, the endwise and sidewise strain responses bound the strain responses for all cask orientations. For cask orientations from $0-90^{\circ}$, the structural strain responses can be linearly interpolated between the sidewise and endwise strain responses. The strain at the inner wall is $0.2 \%\left(S_{1}\right)$ at the impact velocity of $55 \mathrm{mph}$ for sidewise impacts and $38 \mathrm{mph}$ for endwise impacts.

\section{E.6.1.3 IMPASC and NIKE Comparison}

The IMPASC code was benchmarked for endwise impacts at $30 \mathrm{mph}$ on an unyielding surface against the NIKE computer code. Table E.11 summarizes the pertinent results for the representative truck and rail casks. For the truck 
Table E.9

Truck Cask Strain Response to Impact on Unyielding

Surface at Various Cask Orientations

\begin{tabular}{|c|c|c|c|c|}
\hline \multirow{2}{*}{\multicolumn{2}{|c|}{$\begin{array}{c}\text { Cask Orientation } \\
\text { Angle } \\
\left({ }^{\circ}\right)\end{array}$}} & \multicolumn{3}{|c|}{$\begin{array}{c}\text { Strain } \\
(\%)\end{array}$} \\
\hline & & 30 & 38 & 45 \\
\hline & $\begin{array}{l}0 \\
10 \\
30 \\
50 \\
70 \\
90^{\mathrm{a}} /\end{array}$ & $\begin{array}{l}0.175 \\
0.133 \\
0.115 \\
0.107 \\
0.064 \\
0.060\end{array}$ & $\begin{array}{l}0.270 \\
0.210 \\
0.180 \\
0.180 \\
0.081 \\
0.200\end{array}$ & $\begin{array}{l}0.650 \\
0.260 \\
0.255 \\
0.244 \\
0.115 \\
2.00\end{array}$ \\
\hline
\end{tabular}

a/ Includes effects of cab crush and lead slump for $90^{\circ}$ impact 
Table E.10

Rail Cask Strain Response to Impact on Unyielding Surface at Various Cask Orientations

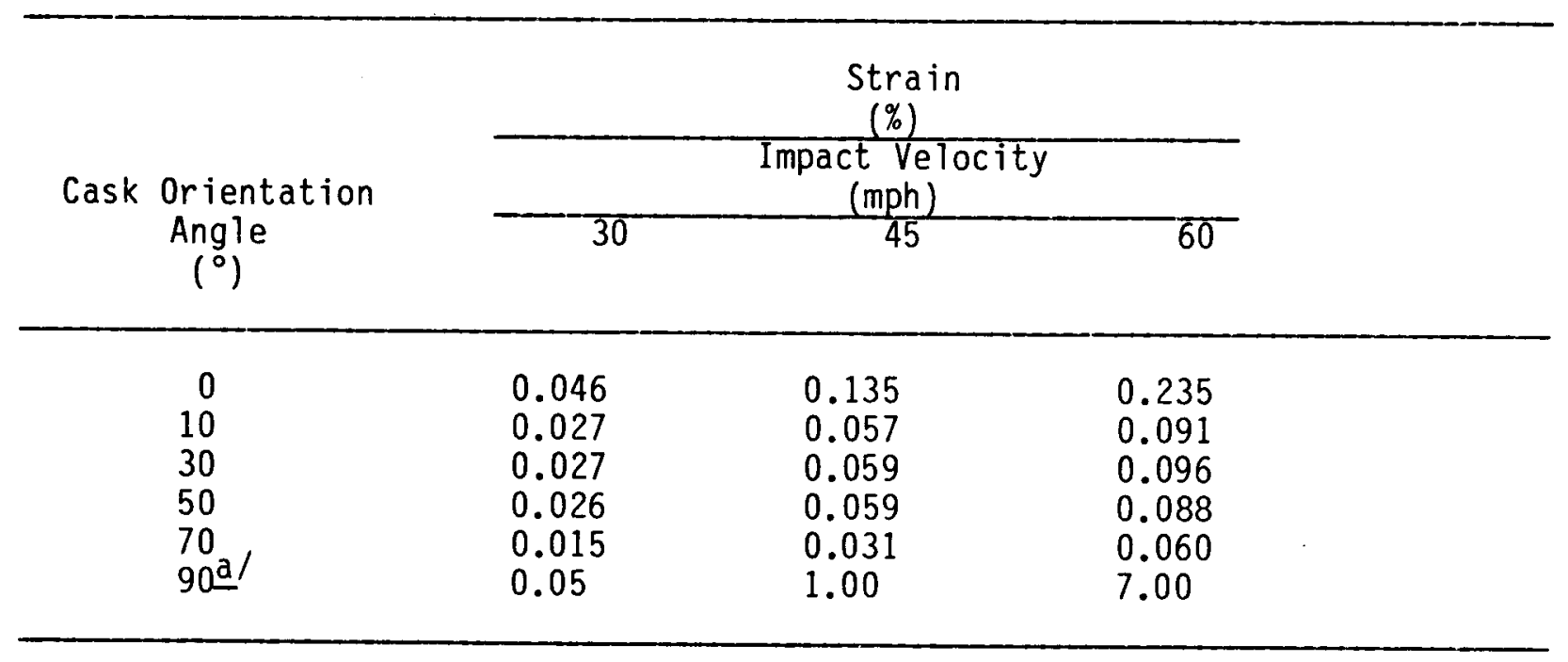

a/ Includes effects of cab crush and lead slump for $90^{\circ}$ impact 
Table E.11

IMPASC Endwise Impact Benchmark Calculation Against NIKE 2-D

\begin{tabular}{|c|c|c|c|c|c|}
\hline & \multicolumn{2}{|c|}{ Truck at $30 \mathrm{mph}$} & \multicolumn{3}{|c|}{$\mathrm{Ra} i 1$ at $30 \mathrm{mph}$} \\
\hline & NIKE 2-D & IMPASC & $\begin{array}{l}\text { Bonded } \\
\text { Elastic } \\
\text { NIKE 2-D }\end{array}$ & $\begin{array}{l}\text { Elastic- } \\
\text { Plastic } \\
\text { NIKE 2-D }\end{array}$ & IMPASC \\
\hline $\begin{array}{l}\text { Force } \\
\text { (g) }\end{array}$ & 37.5 & 45.0 & 36 & 36 & 28.6 \\
\hline $\begin{array}{l}\sigma \\
\text { axial } \\
\text { (psi) }\end{array}$ & -9543 & -12200 & -6732 & -12035 & -7100 \\
\hline $\begin{array}{l}\text { Maximum } \\
\text { deflection } \\
\text { of } 7 \text { imiter } \\
\text { (inches) }\end{array}$ & 25.8 & 26.5 & 25.3 & 25.8 & 26.5 \\
\hline $\begin{array}{l}\text { Maximum } \\
\text { plastic } \\
\text { strain or } \\
\text { effective } \\
\text { strain if } \\
\text { elastic } \\
\left({ }^{\circ}\right)\end{array}$ & 0.00077 & $N / A$ & 0.00038 & 0.0012 & N/A \\
\hline
\end{tabular}


cask calculations, the material properties of Section E.2 and cask configuration of Fig. E-15 were used. In the NIKE calculation the lead was assumed to be unbonded from the stainless steel shells, whereas in the IMPASC calculation the lead was assumed to be bonded. The calculated impact force was approximately $38 \mathrm{~g}$ at $30 \mathrm{mph}$ and the impact limiter deflection was approximately 26 inches in both.calculations. Rail cask calculations were made with NIKE for bonded and unbonded lead. The results for the bonded lead are in good agreement with the IMPASC results which are also based on the assumption of bonded Tead. The effect of assuming the lead unbonded is primarily an increase of the stress and strain on the inner shell of the cask caused by the lead pressure. From this benchmark comparison it was concluded that significant lead slump would not occur and the plastic strain is less than $0.2 \%\left(S_{1}\right)$ when the axial impact force on the cask is less than $40 \mathrm{~g}$.

\section{E.6.2 Elastic-Plastic Response by Cask}

Elastic-plastic calculations were necessary when cask impact forces exceeded $40 \mathrm{~g}$. Several of the capabilities of the DYNA and NIKE finite element codes that are critical to such calculations are (1) elastic-plastic material models, (2) sliding interfaces, (3) dynamic solutions, and (4) the ability to solve large deformation problems. The cask models include a 304 stainless steel inner wall, lead shielding and a 304 stainless steel outer wall. Each of the materials was modeled as a bilinear elastic-plastic material. The material properties used are summarized in Section E.2. The calculations were performed, for endwise, and sidewise impacts. The cask responses to impacts at other cask orientations are assumed to be bounded by the endwise and sidewise response results.

\section{E.6.2.1 Endwise Impacts}

Endwise impact"calculations were performed for the representative truck and rail casks striking an unyielding surface. The caskiswere dropped from several heights onto an unyielding surface to obtain their responses over a range of impact conditions. The casks were modeled as 2-D axisymmetric composite cylinders with closures as shown in Fig. E-5. MAZE was used to 
generate the finite element meshes. DYNA 2-D/NIKE 2-D were used to perform the impact calculations. E.15

\section{E.6.2.1.1 Truck Cask Impact}

The truck cask was modeled using two elements for the inner shell as shown in Fig. E-11. The results of the endwise impact calculations are summarized in Table E.12. The sudden deceleration of impact caused the lead shielding to slump and the cask length to decrease as shown in Fig. E-12 for the $60 \mathrm{mph}$ impact. The maximum strain conditions occur at the inner wall at the flange joint as shown in Fig. E-13 for the $60 \mathrm{mph}$ impact. The velocity changes with time, or decelerations, of the steel structure and the lead shielding were significantly different as shown in Fig. E-14 for the $60 \mathrm{mph}$ impact. Al1 impact calculations were terminated after rebound occurred. The lead slump is determined by finding the void between the cask stee 1 body and lead shield. For example, consider a truck cask impacting at $60 \mathrm{mph}$. The time for the lead and the steel to reach zero velocity is extrapolated from Fig. E-14 as 19 msecs. Then the curves on Fig. E-12 are extrapolated to 19 msecs. This gives 16.5 inches of axial displacement at the top of the lead, and 4.2 inches in the steel at the top of the lead cavity. The relative displacement is the lead slump, and is (16.5 - 4.2) 12.3 inches.

An average interface deceleration force was calculated for the cask at each impact velocity by averaging the interface force over the time it took the steel structure to come essentially to a stop as shown in Fig. E-14. For example, consider the truck cask impacting at $60 \mathrm{mph}$. The time for the total steel mass to come nearly to a stop is 6 msecs as determined from Fig. E-14. The stee 1 interface force acting for the first $6 \mathrm{msec}$ of impact ranges from a high of $373 \mathrm{~g}$ to a low of $143 \mathrm{~g}$, therefore the average interface force is the sum of the forces divided by 2 or $258 \mathrm{~g}$. The average interface deceleration force was used to estimate the cask response to impacts on real surfaces as discussed in Section E.7. 


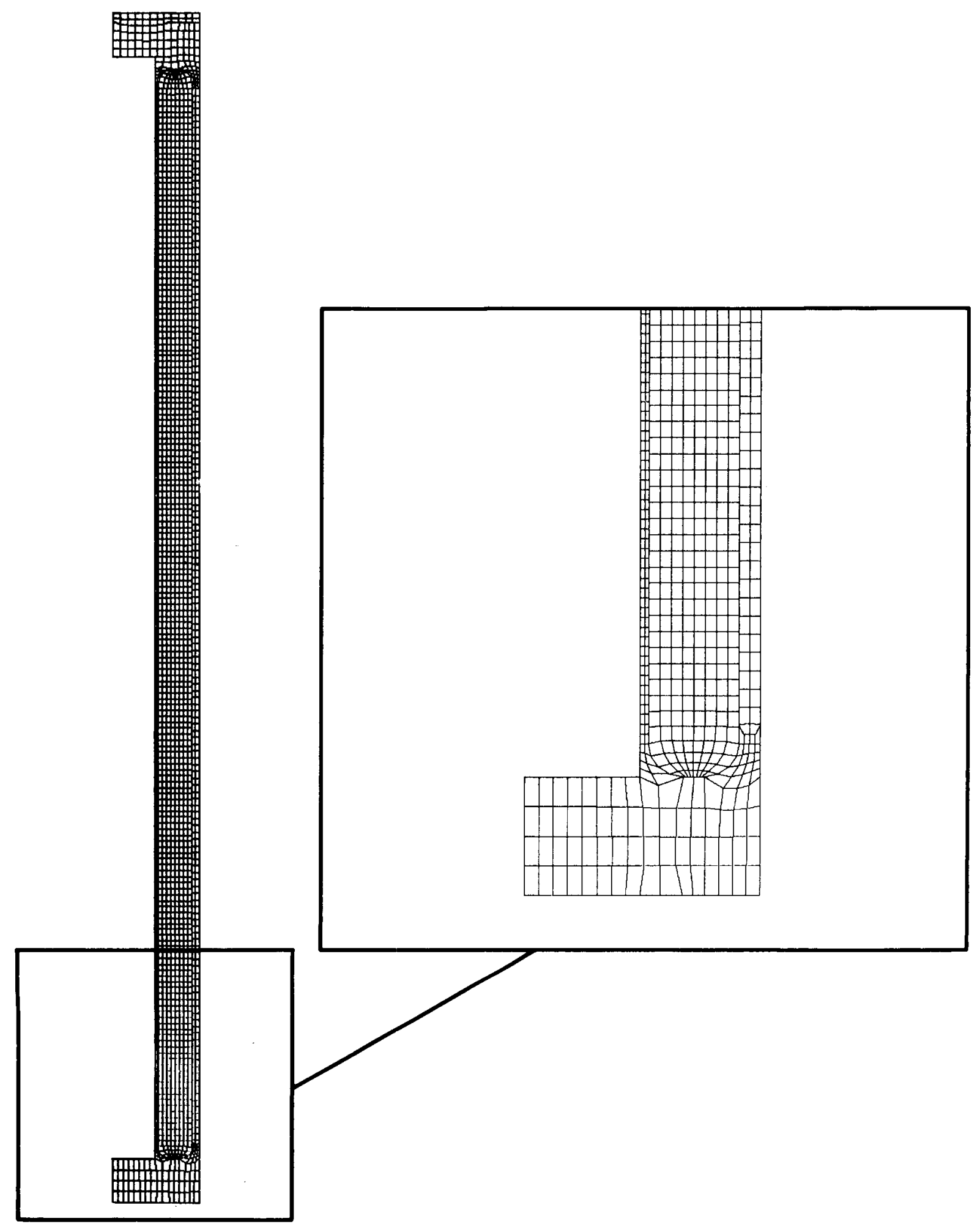

Figure E-11 Finite element mesh for two-element inner-wall model by truck cask. 
Table E.12

Summary of Truck Cask Endwise Impact Resultsal

\begin{tabular}{lccc}
$\begin{array}{c}\text { Velocity } \\
\text { (mph) }\end{array}$ & $\begin{array}{c}\text { Interface } \\
\text { Force } \\
(\mathrm{g})\end{array}$ & $\begin{array}{c}\text { Strain } \\
\text { Inner She } 11 \\
(\%)\end{array}$ & $\begin{array}{c}\text { Lead } \\
\text { Slump } \\
\text { (in) }\end{array}$ \\
\hline 30 & 38 & 0.077 & 0 \\
45 & 90 & 3.60 & 4 \\
$60 \mathrm{~b} /$ & 258 & 23.3 & 12.3 \\
90 b & 353 & 36.2 & 24 \\
\hline
\end{tabular}

a/ Cab crush not included in analysis.

b/ Impact limiter not included in analysis. 


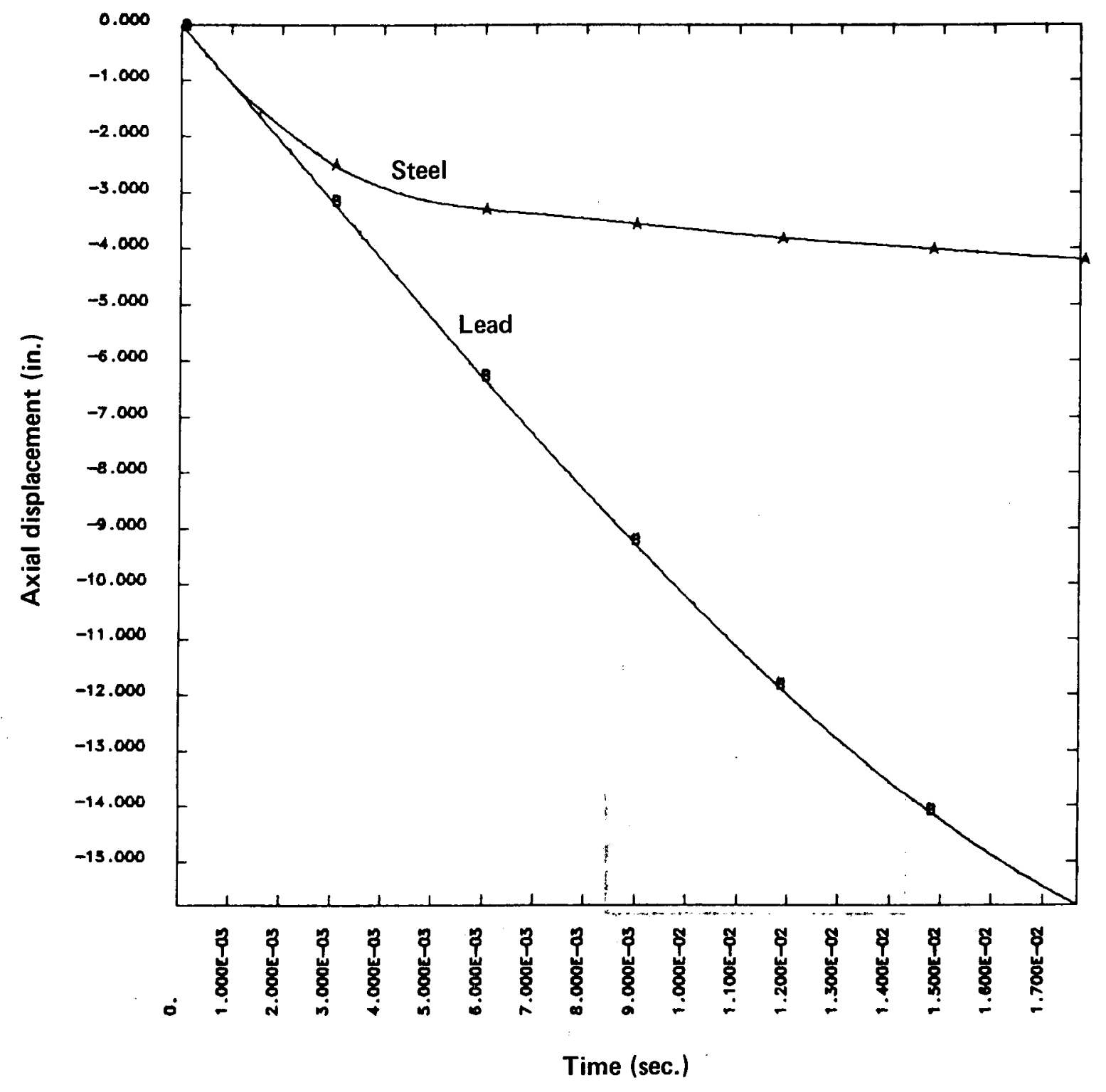

Figure E-12 Lead slump in truck cask at $60 \mathrm{mph}$ impact. 
$\operatorname{Min}(-)=0$

$\operatorname{Max}(+)=2.33 \mathrm{E}-01$

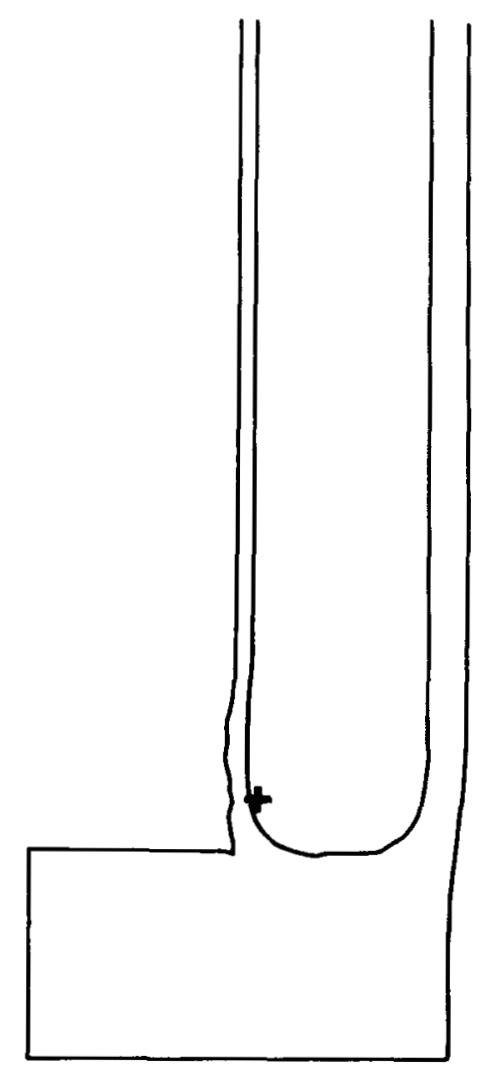

Figure E-13 Strain in lower steel structure for truck cask impact at $60 \mathrm{mph}$.

$$
E-40
$$




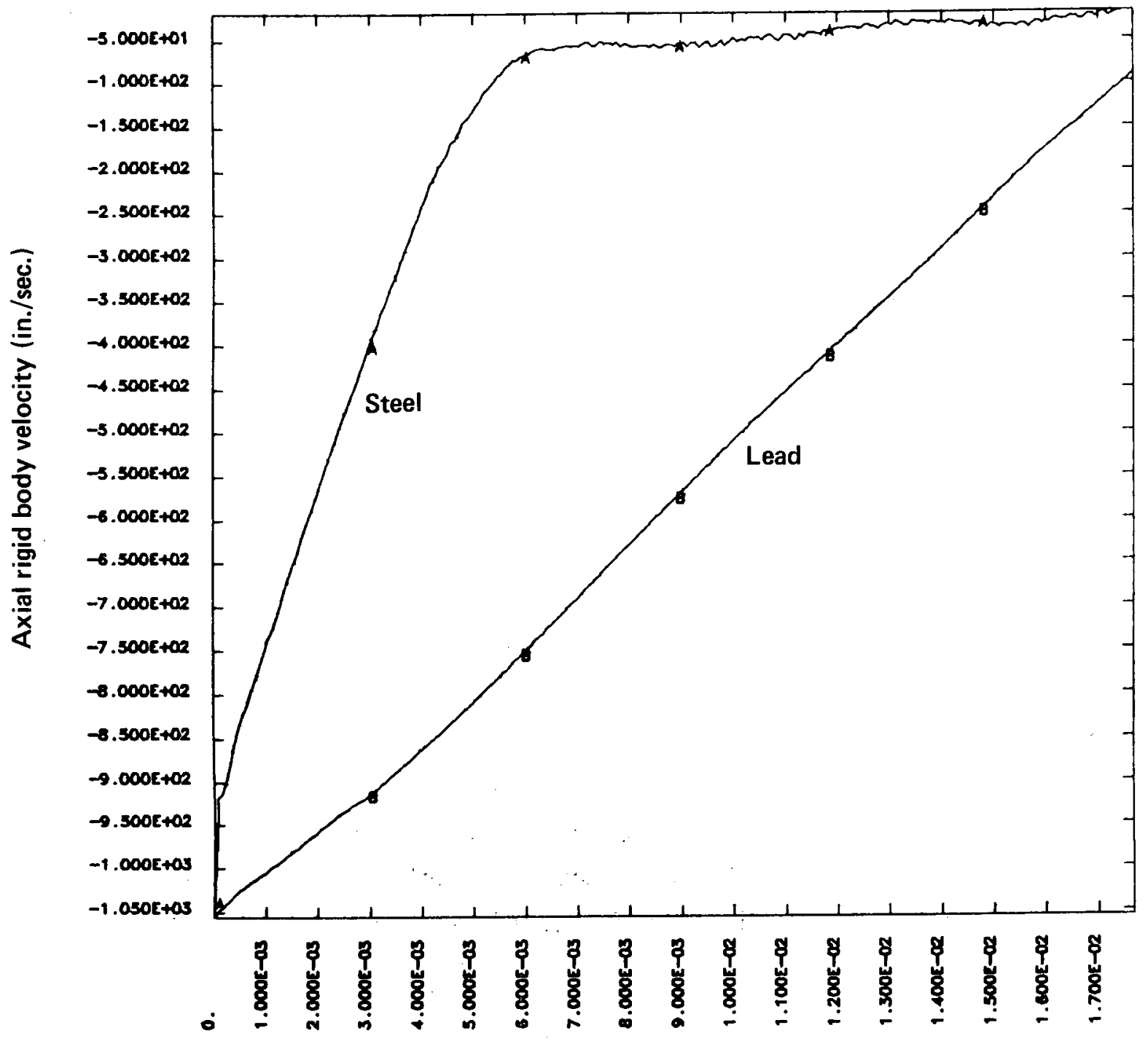

Time (sec.)

Figure E-14 Velocity versus time for truck cask impact at $60 \mathrm{mph}$. 


\section{E.6.2.1.2 Rail Cask Impact}

The rail cask was modeled using two elements for the inner shell as shown in Fig. E-15. The results of the endwise impact calculations are summarized in Table E.13. The lead slump that occurred in the rail cask is shown in Fig. E-16 for the $90 \mathrm{mph}$ impact. The strain condition in the steel structure at the end of impact is shown Fig. E-17 for the $90 \mathrm{mph}$ impact. The velocity change, for the steel structure and lead shielding is shown in Fig. E-18. The average interface deceleration force was calculated from Fig. E-18 for the $90 \mathrm{mph}$ impact with the method discussed in Subsection E.6.2.1.1 for the truck cask.

\section{E.6.2.2 Sidewise Impacts}

Two-dimensional plane strain analyses without impact limiters or end enclosures were performed for sidewise impacts on an unyielding surface to estimate the 3-D responses for the casks. This approximate 2-D method overestimates strain responses of the representative casks, particularly for impact velocities less than $60 \mathrm{mph}$ and for impacts on soft surfaces such as soil. The 2-D method was benchmarked in subsection E.6.2.2.3 with a 3-D impact analys is that modeled the representative truck cask with impact limiters and end closures. This approximate method eliminates the need to perform a series of 3-D sidewise impact analyses.

The 2-D truck cask models were developed using the SLIC interactive mesh generator. E.16 The dimensions in the SLIC command file were modified to generate the rail cask models. The cask models do not include contents. DYNA 2-D (2), an explicit 2-D hydrodynamic finite element program, was used to do the plane strain analysis.

\section{E.6.2.2.1 Truck Cask Impact}

For unyielding surface impacts, a vertical symmetry plane was used in the modeling as shown in Fig. E-19 to reduce the solution cost. The calculations were performed for three different truck cask initial velocities: $30 \mathrm{mph}, 60$ $\mathrm{mph}$, and $90 \mathrm{mph}$. The calculations were terminated when the cask started to 

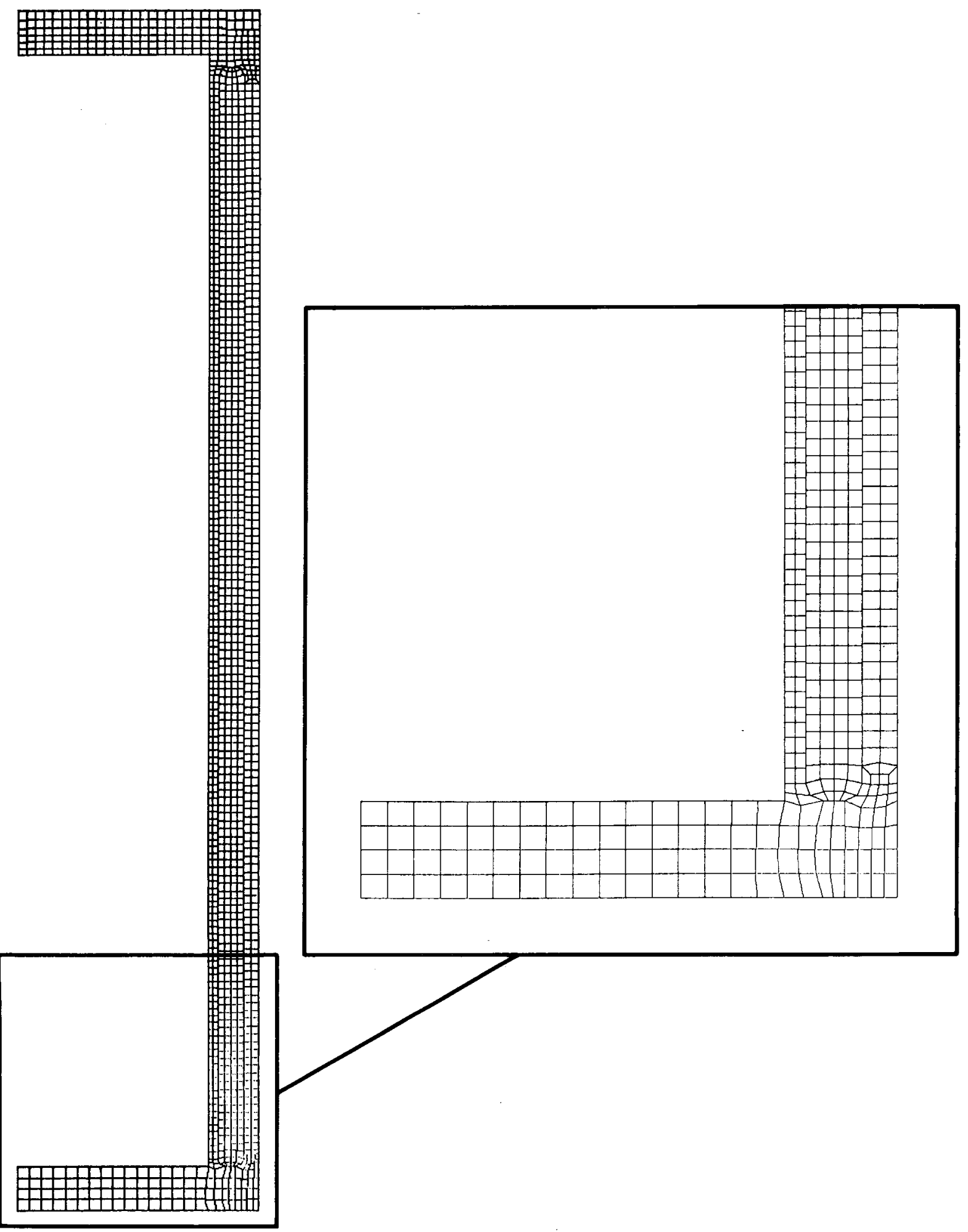

Figure E-15 Finite element mesh for rail cask.

$$
E-43
$$


Table E.13

Summary of Rail Cask Endwise Impact Resultsal

\begin{tabular}{cccc}
$\begin{array}{c}\text { Velocity } \\
(\mathrm{mph})\end{array}$ & $\begin{array}{c}\text { Interface Deceleration } \\
\text { Force } \\
(\mathrm{g})\end{array}$ & $\begin{array}{c}\text { Strain } \\
\text { Inner Wa11 } \\
(\%)\end{array}$ & $\begin{array}{c}\text { Lead } \\
\text { Slump } \\
(\text { in })\end{array}$ \\
\hline 30 & 36 & 0.12 & 0.5 \\
45 & 103 & 1.9 & 6.0 \\
90 b & 425 & 24.3 & 24.8 \\
\hline
\end{tabular}

a/ Cab crush not included in analysis.

b/ Impact limiter not included in analysis. 


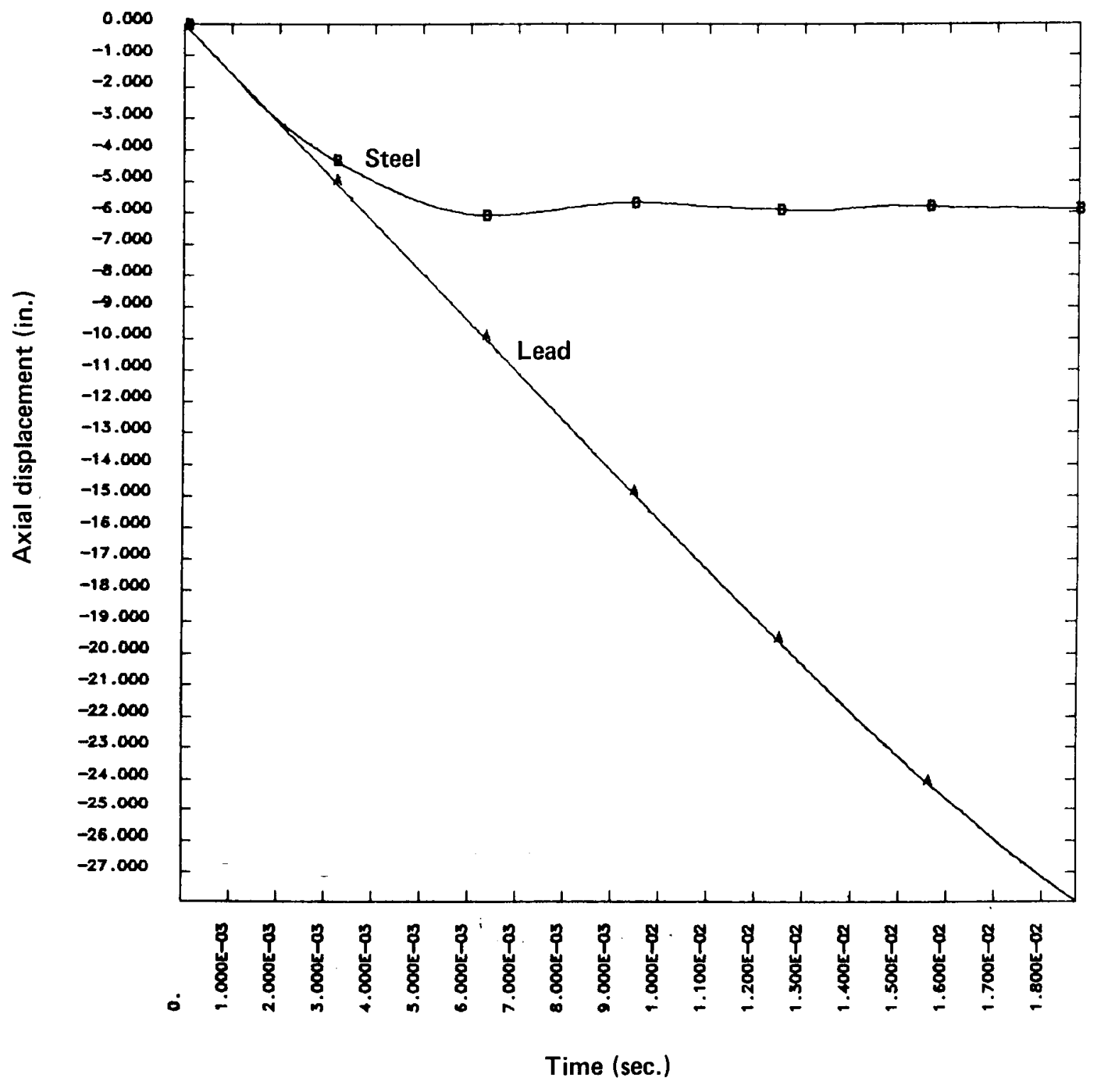

Figure E-16 Lead slump in rail cask at $90 \mathrm{mph}$ impact. 
$\operatorname{Min}(-)=0$

$\operatorname{Max}(+)=2.43 E-01$

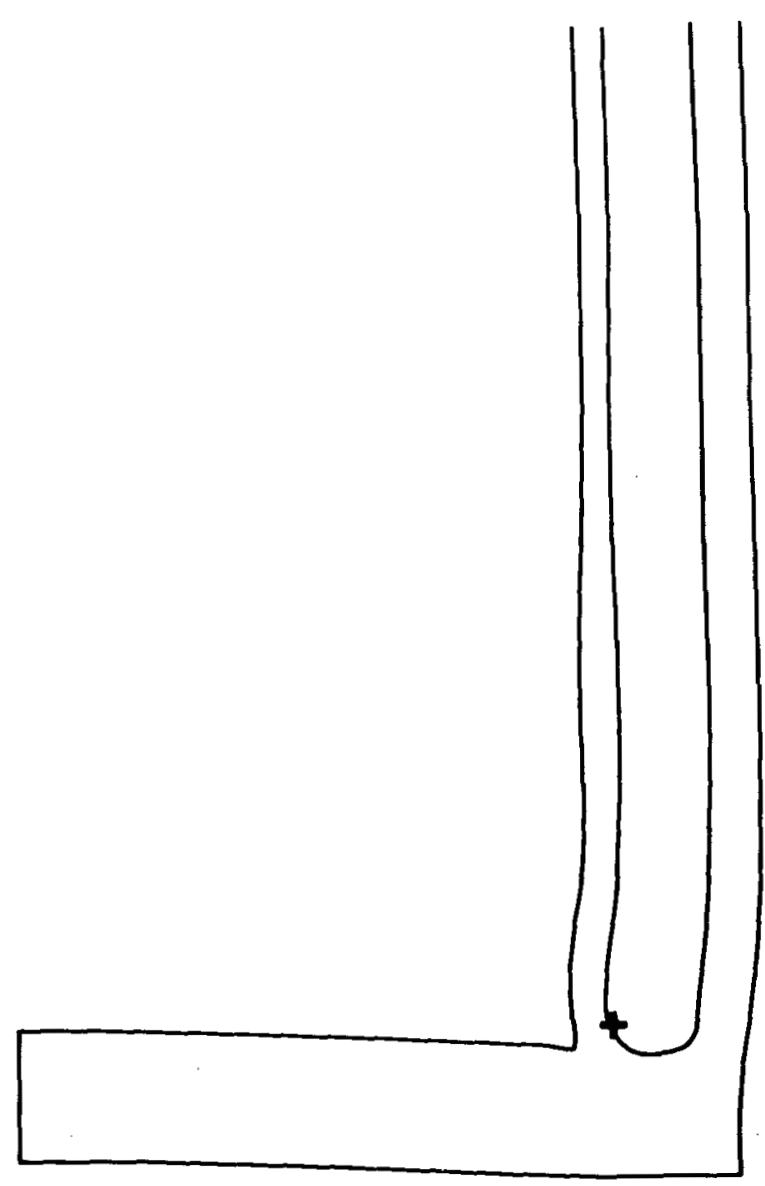

Figure E-17 Strain in lower steel structure for rail cask impact at $90 \mathrm{mph}$. 


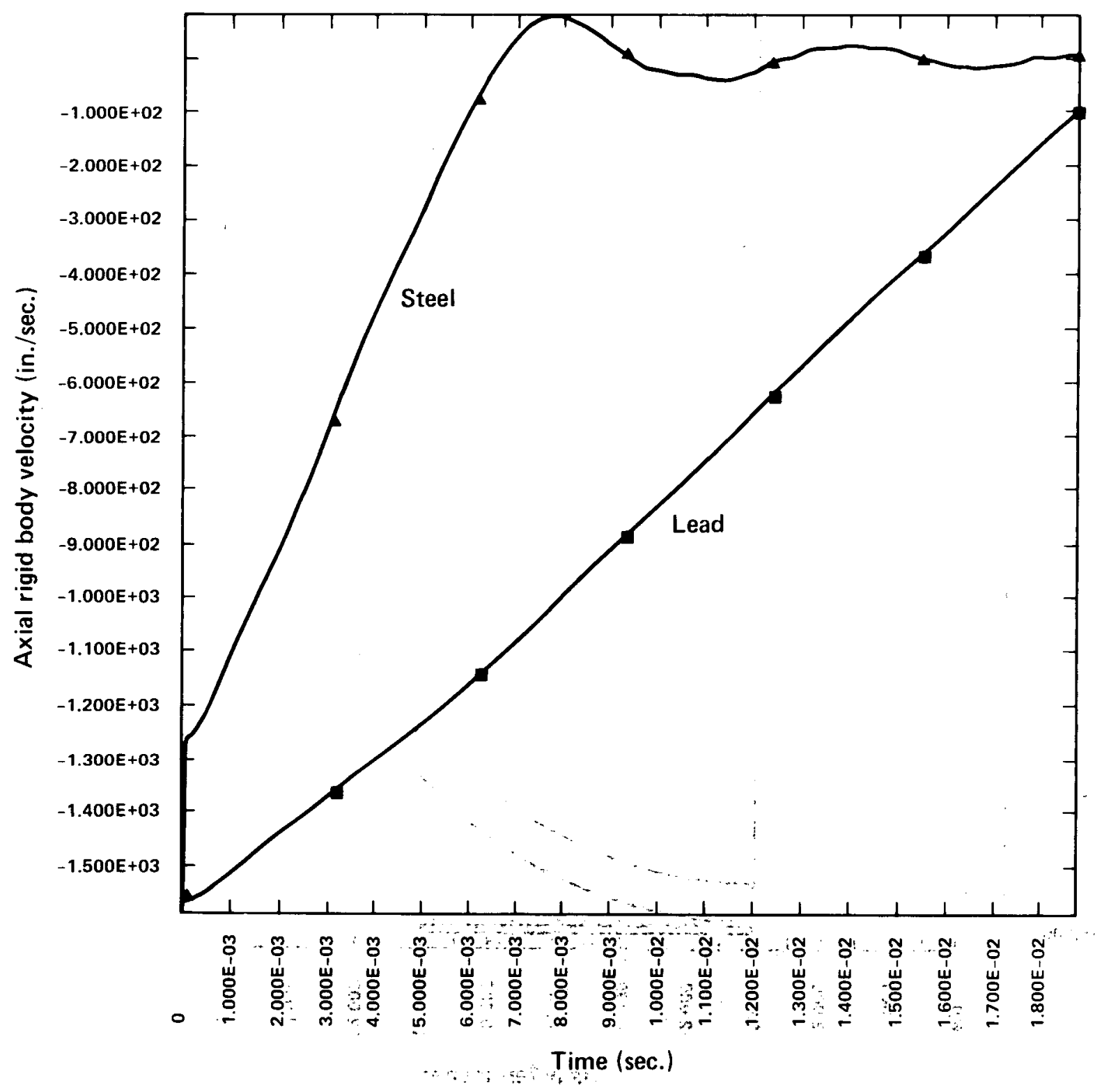

Figure E-18 Velocity versus time for rail cask impact at $90 \mathrm{mph}$. 


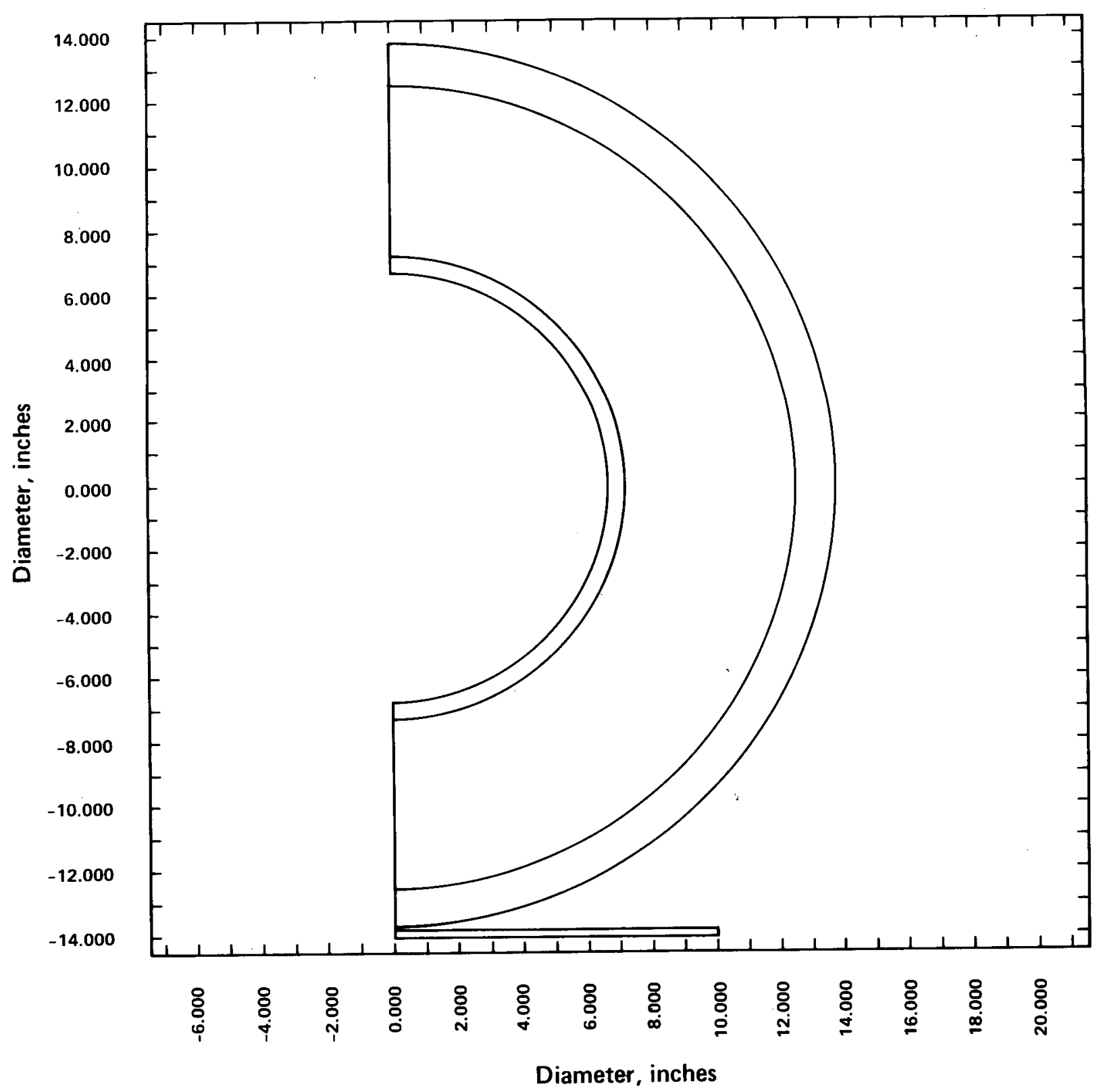

Figure E-19 Model of a truck cask impacting an unyielding surface. 
rebound. The sudden deceleration caused the cask to flatten as shown in Fig. E-20.

For the $30 \mathrm{mph}$ impact, the cask experienced $160 \mathrm{~g}^{\prime} \mathrm{s}$ and sustained a maximum effective stress of 36,000 ps $j$ and maximum plastic strain of $5.9 \%$ in the steel shel1s. For $60 \mathrm{mph}$, the $\mathrm{g}$ 's increased to 342, the maximum effective stress increased to 45,300 ps $i$ and the maximum plastic strain increased to 14\%. These results are summarized in Table E.14. The location of the maximum plastic strain is shown in Fig. E-21 for the 60 mph impact.

\section{E.6.2.2.2 Rail Cask Impact}

Calculations were performed for the rail cask with initial velocities of $30 \mathrm{mph}$ and $60 \mathrm{mph}$. The $30 \mathrm{mph}$ calculation was terminated when the cask started to rebound. The $60 \mathrm{mph}$ calculation was terminated when the cask started to fold on itself. The sudden deceleration caused the cask to flatten considerably and, in the $60 \mathrm{mph}$ case, to develop a plastic hinge as shown in Fig. E-22. The cask contents would to some degree resist the formation of the plastic hinge. However, the cask contents were not modeled.

For the $30 \mathrm{mph}$ impact, the cask experienced a force of $29 \mathrm{~g}$ 's and sustained a maximum effective stress of 32,400 psi and maximum plastic strain of $4.1 \%$ in the steel shells. For $60 \mathrm{mph}$, the $\mathrm{g}$ 's increased to 47 , the maximum effective stress increased to 37,400 psi and the maximum plastic strain increased to $7.2 \%$. These results are summarized in Table E.15. The location of the maximum plastic strain is shown in Fig. E-23 for the 60 mph impact.

\section{E.6.2.2.3 Three-Dimensional Sidewise Impact}

A 3-D truck shipping cask was modeled for the side-drop analys is with impact limiters. As shown in Fig. E-24, the model includes the inner and outer steel shells, the lead shielding, the steel end caps, and the balsa wood impact limiters. The finite element model was generated using SLIC, an interactive mesh generator. The impact limiters were not tied to the cask, conservatively allowing them to slide relative to the cask because any bolt retaining forces that could be present are unknown. Two planes of symmetry 

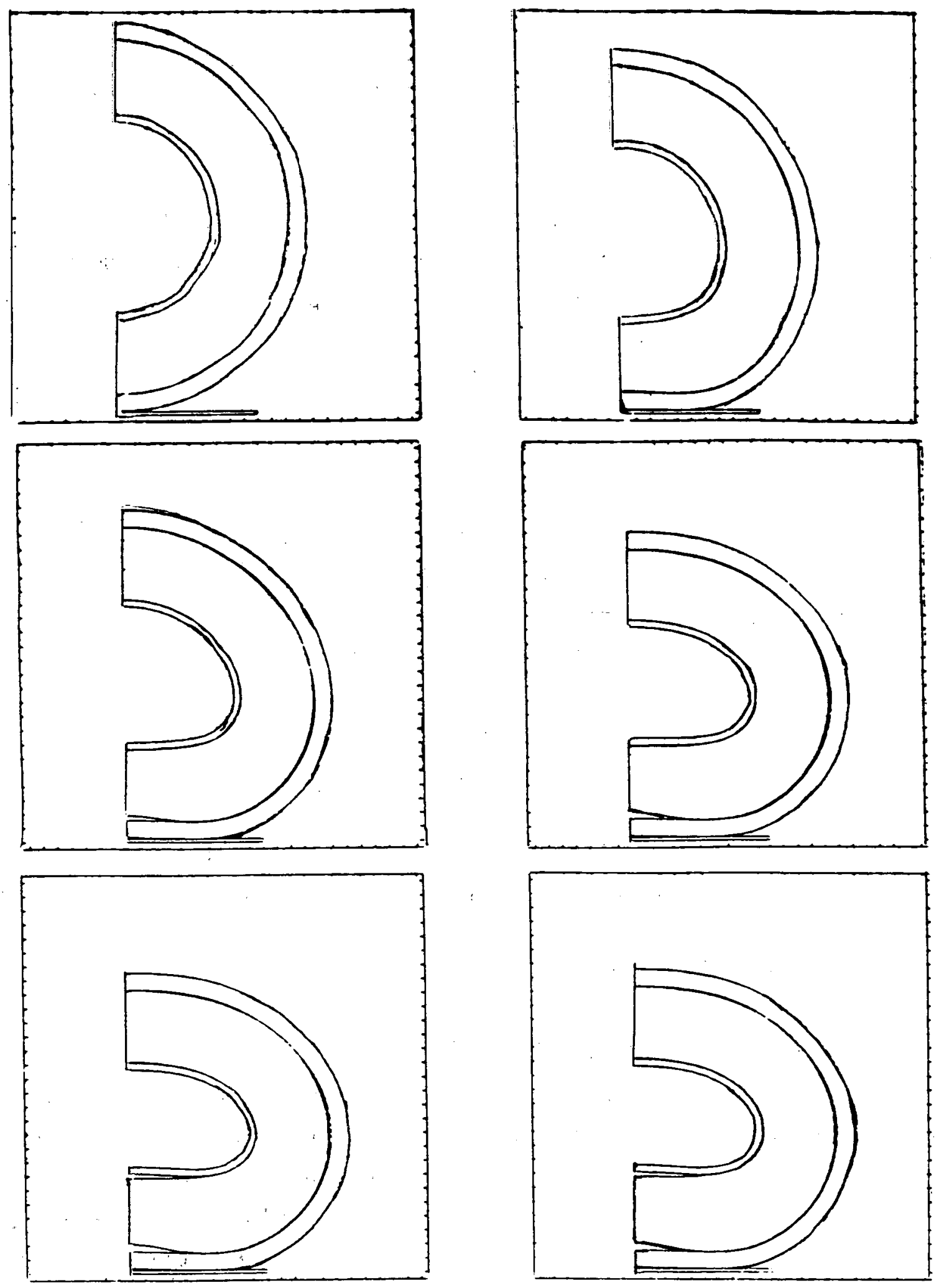

Figure E-20 Truck cask impact on unyielding surface at $60 \mathrm{mph}$.

$$
E-50
$$


Tabile E. 14

Results of Truck Cask Sidewise Impact on an Unyielding Surface

Cask impact velocity (mph)

Time at which rebound starts (sec)

g load on cask (g)

Maximum effective stress (psi)

Maximum plastic strain (\%)

$\begin{array}{rrr}30 & 60 & 90 \\ 0.0085 & 0.008 & 0.0075 \\ 160 & 342 & 547 \\ 36,000 & 45,300 & 63,100 \\ 5.9 & 14 . & 23.1\end{array}$

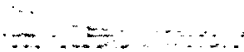

$E-5 \cdot 1$ 
$\operatorname{Min}(-)=0$

$\operatorname{Max}(+)=1.40 E-01$

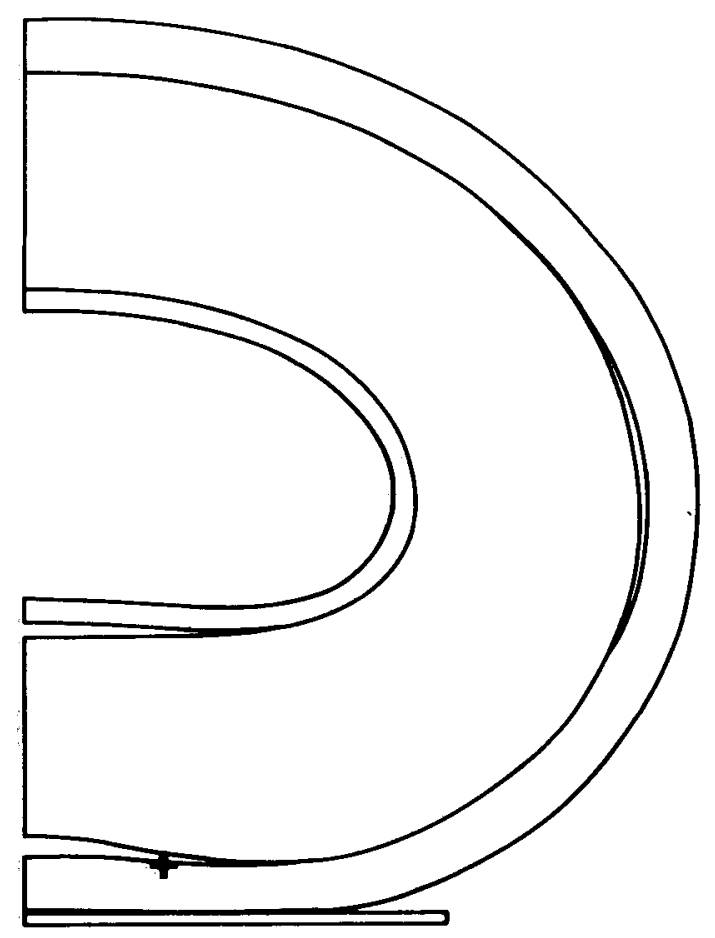

Figure E-21 Impact on unyielding surface at $60 \mathrm{mph}$ - maximum plastic strain location. 

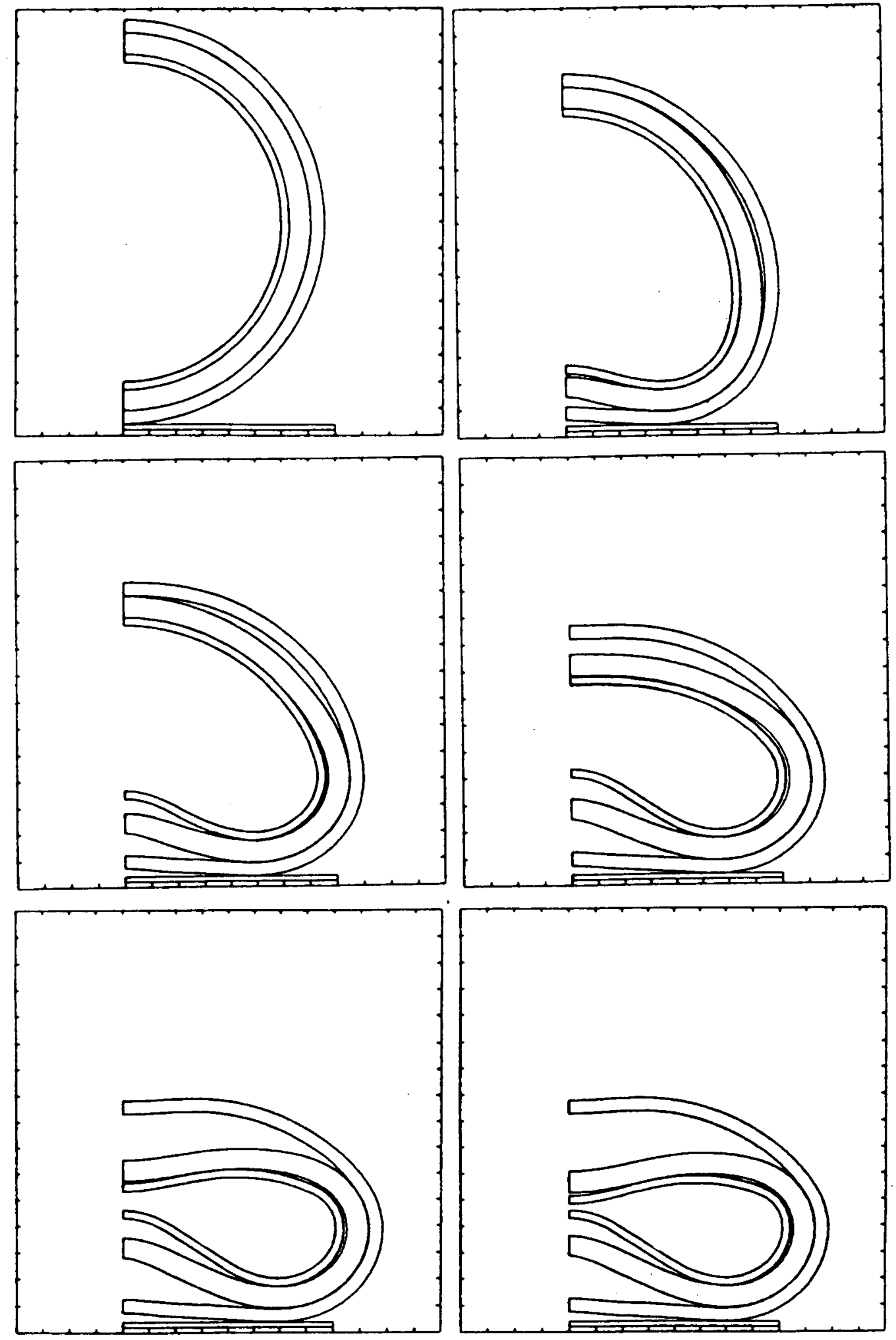

Figure E-22 Rail cask impact on unyielding surface at $60 \mathrm{mph}$. 
Table E. 15

Results of Rail Cask Sidewise Impact on an Unyielding Surface

\begin{tabular}{lrr}
\hline Cask impact velocity (mph) & 30 & 60 \\
Time at which rebound starts (sec) & 0.048 & N/A \\
g load on cask (g) & 29 & 47 \\
Maximum effective stress (psi) & 32,400 & 37,400 \\
Maximum plastic strain (\%) & 4.1 & 7.2 \\
\hline
\end{tabular}


$\operatorname{Min}(-)=0$

$\operatorname{Max}(+)=7.20 E-02$

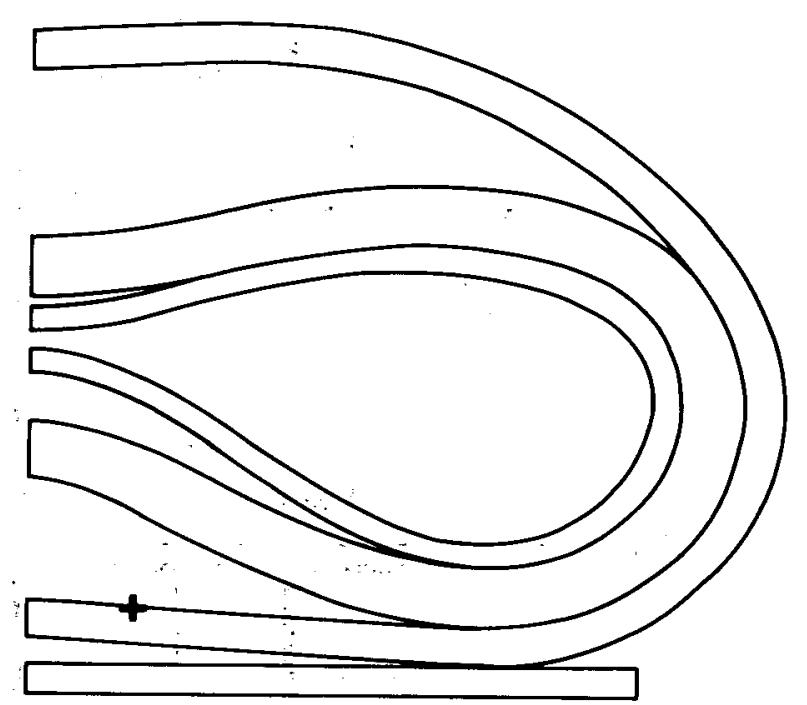

Figure E-23 Rail cask impact on unyielding surface at $60 \mathrm{mph}$ - maximum plastic strain location. 


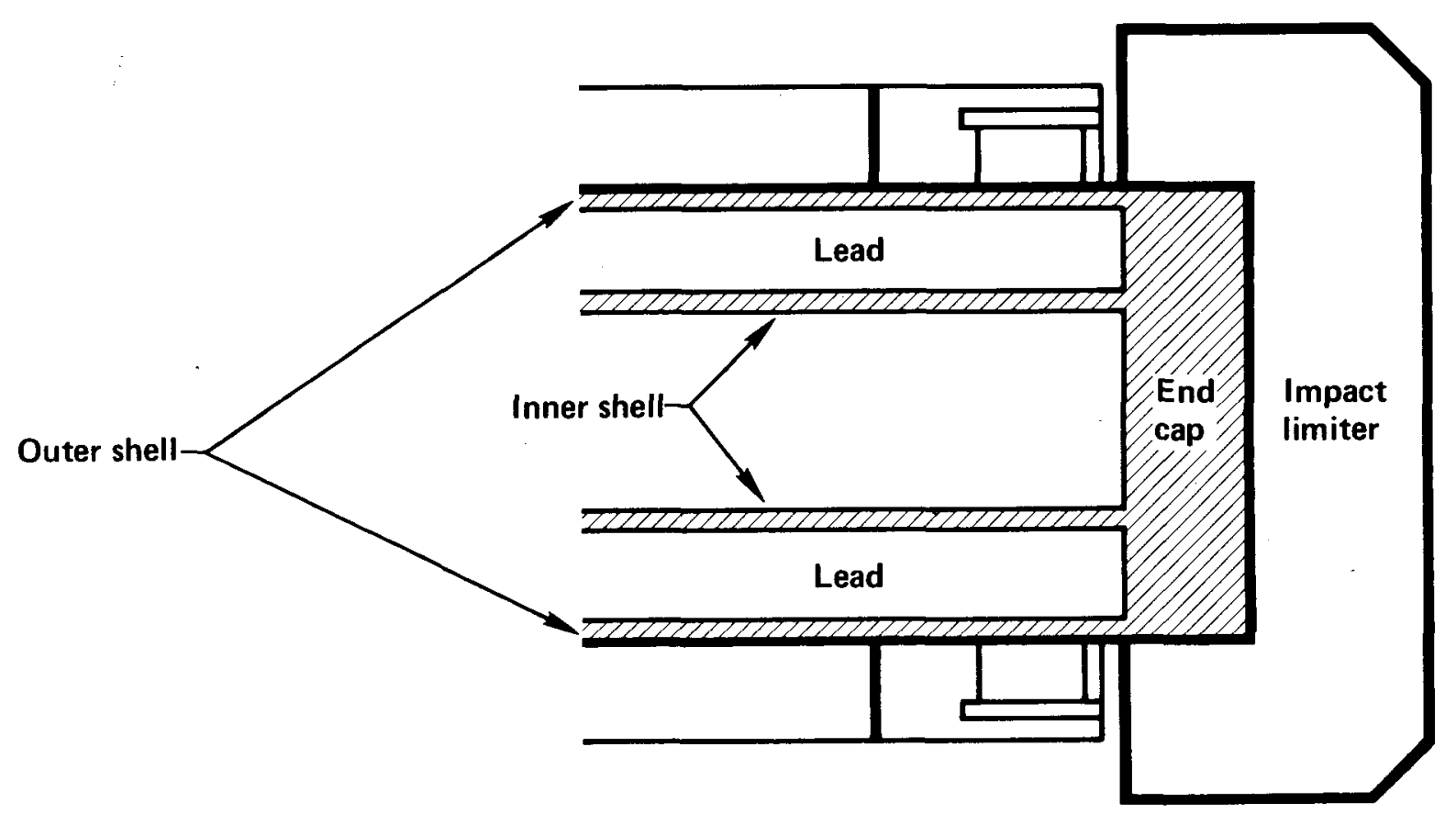

Figure E-24 Full side drop geometry including impact limiters. 
were incorporated to reduce the model's complexity. The inner and outer steel shells were modeled using the thick shell option in DYNA 2-D.

The impact velocity was $60 \mathrm{mph}$, resulting in deceleration of $108 \mathrm{~g}$ 's and the deformation shown in Fig. E-25. The cask bowed because it was supported by the impact limiters around the end caps. The center of the cask impacted the unyielding surface at almost $60 \mathrm{mph}$. The contact area increased to approximately half the length of the cask when impact was complete and rebound started to occur. The strain distribution shown in Fig. E-26 indicates that the maximums occur at the center of the cask. The maximum effective stress was 42,500 psi; the maximum plastic strain was $8.7 \%$; and the maximum shear stress was 24,400 psi.

The calculation of the full side-drop with impact limiters showed several things. First, the cask bows when the ends impact first because of the impact limiters. Second, as the cask bows and the center of the cask impacts the unyielding surface, the center of the cask is still traveling at almost full speed. The bowing does not slow down the center of the cask.

A thin slice of the cask was isolated at the center and compared with a 2-D plane strain calculation with the same impact velocity of $60 \mathrm{mph}$. The deformations are virtually the same as shown in Fig. E-27. The stresses and strains also compared favorably. Since the deformed slopes compared so closely, it was concluded that 2-D calculations can be used to represent 3-D impacts on surfaces at $60 \mathrm{mph}$ and greater.

\section{E.7 Impacts on Real Objects}

Ideally, it would be desirable to perform the response calculations assuming both representative casks and real impact surfaces. This can be done using either DYNA 2-D/3-D or NIKE 2-D/3-D computer codes. However, given that computer runs have to be performed to cover many variations in cask orientation angle, surface hardness, and impact velocities, expense precludes the use of DYNA or NIKE codes for each case. 

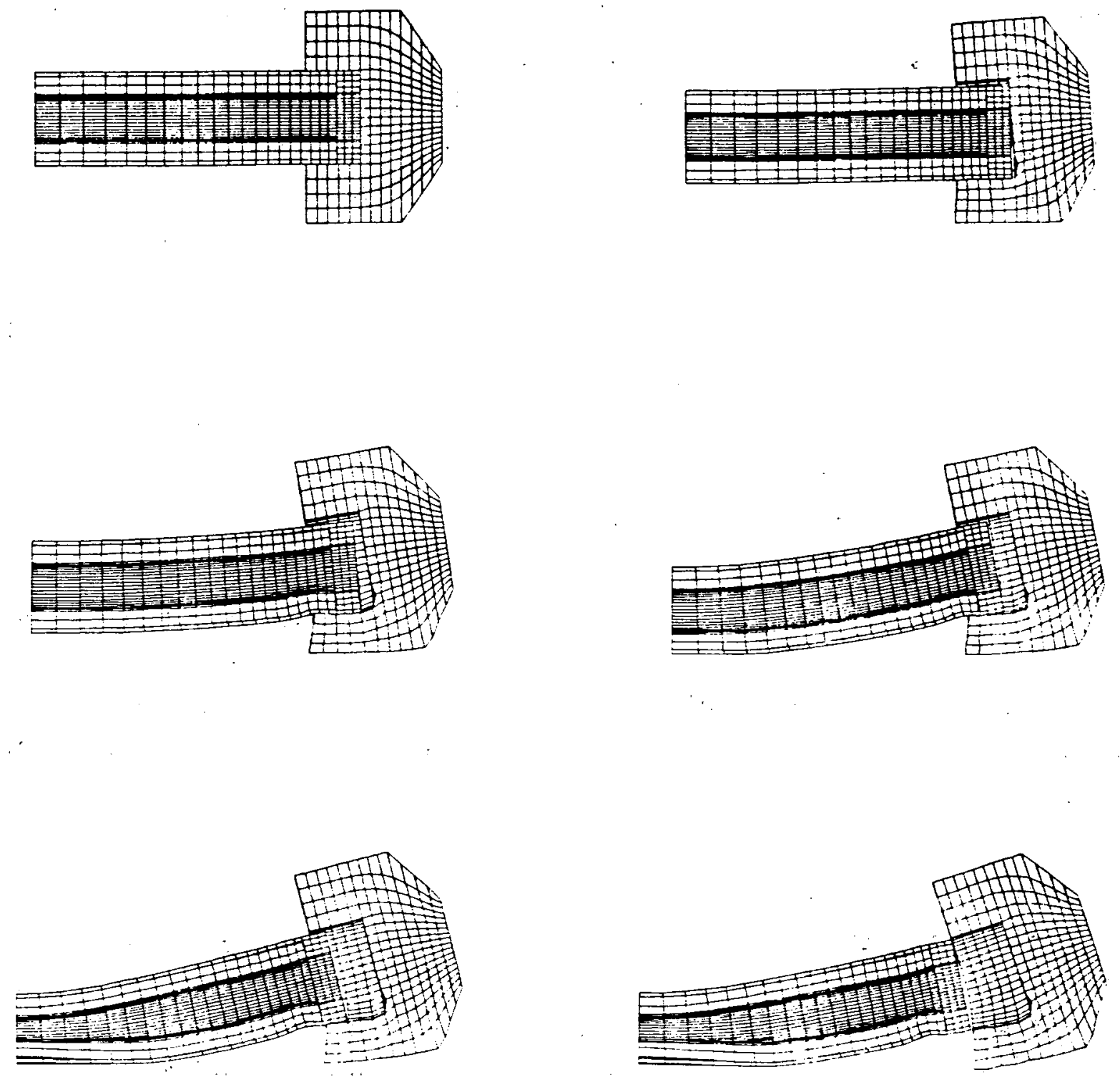

Figure E-25 Deformations of truck cask during $60 \mathrm{mph}$ side drop (side view) 
time $2.99004 e-02$

contours of eff. plastic strain

$\begin{array}{ll}\min : 0.686 \mathrm{in} \text { element } & 3561 \\ \max \cdot 8.686 \mathrm{in} & \text { element } 3163\end{array}$

$$
\begin{gathered}
\text { contour values } \\
\text { a: } 8.69 \mathrm{e}-03 \\
b=1.74 \mathrm{e}-02 \\
\mathrm{c}=2.61 \mathrm{e}-02 \\
\mathrm{~d}=3.47 \mathrm{e}-02 \\
\mathrm{e}=4.34 \mathrm{e}-02 \\
\mathrm{f}=5.21 \mathrm{e}-02 \\
9=6.08 \mathrm{e}-02 \\
h=6.95 \mathrm{e}-02 \\
\mathrm{i}=7.82 \mathrm{e}-02
\end{gathered}
$$

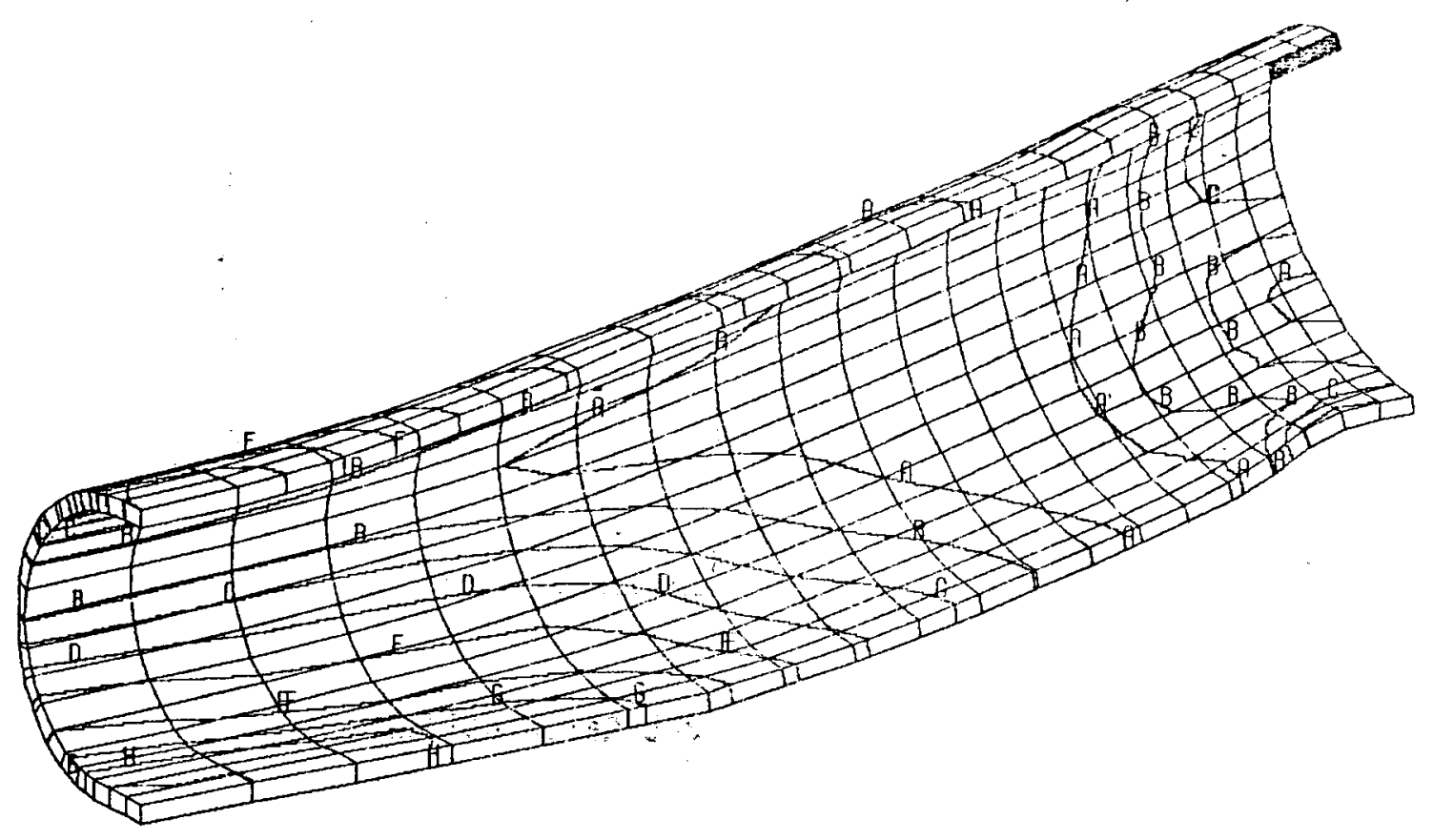

Figure E-26 Distribution of plastic strain at end of impact (outer she11). 


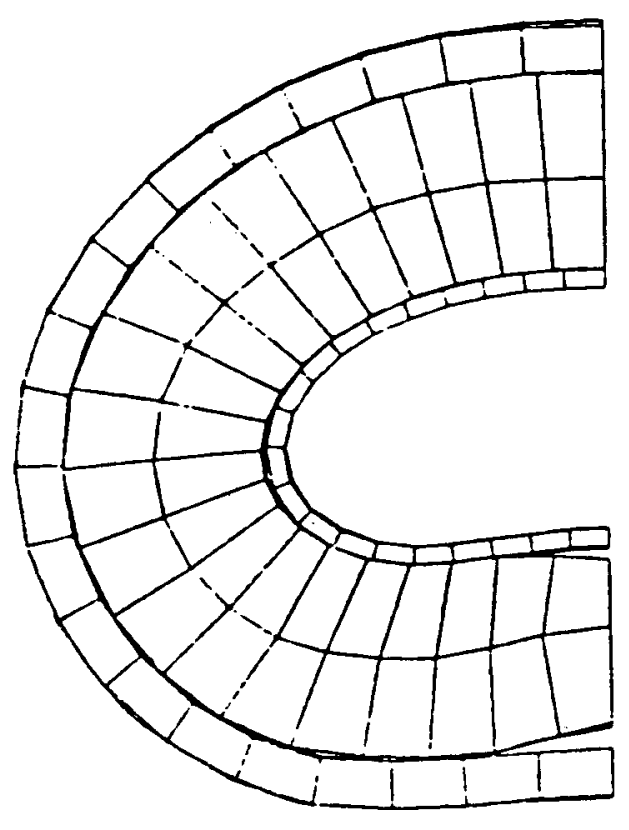

3-D Calculations

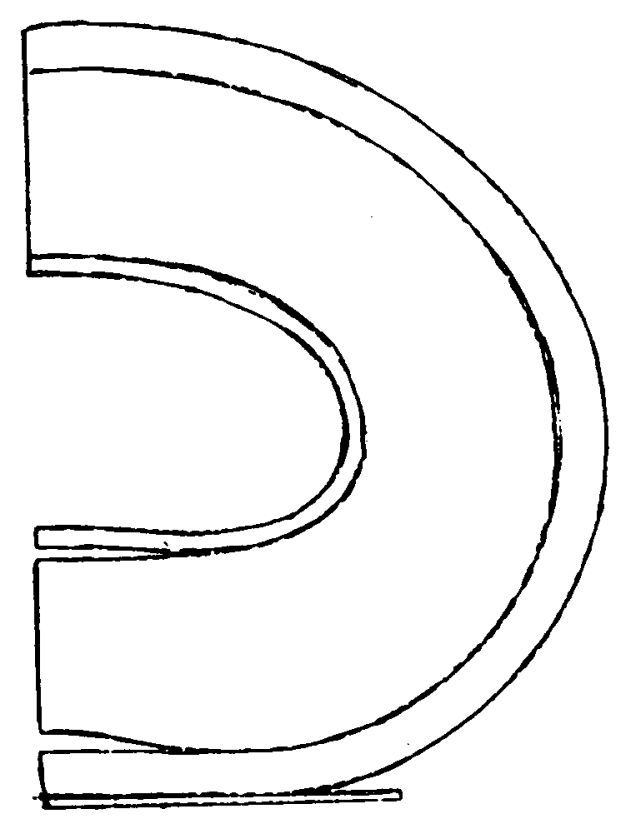

2-D Calculations

Figure E-27 Comparison of 2-D deformations with 3-D deformations at the center of the cask. 
To simplify the otherwise massive finite element analyses necessary to analyze a representative, i.e., a deformable cask impacting a deformable surface, an equivalent damage technique was devised. Using the equivalent damage technique described in Subsection E.7.1, the cask response was estimated for impacts on real surfaces.

\section{E.7.1 Equivalent Damage Technigue}

In the equivalent damage technique, the total deformation, and thus the total energy absorption caused by impact, is divided into two parts. The basic assumption is that the total energy of the falling cask is absorbed by deformation of the cask itself and the surface that it hits. In order to estimate how much of the energy is absorbed by the surface, the cask is modeled as a rigid body, and the surface is modeled as an energy-absorbing medium. Using this model, the impact force on the rigid cask can be determined for several velocities. In order to accomplish the necessary analyses, the characteristics of several real target surfaces must be determined.

The energy absorbed by the cask itself is estimated by modeling a deformable cask impacting an unyielding surface. Impact forces and corresponding cask deformations are determined for different impact velocities using this model. In a real situation both the cask and surface would deform. Taking the deformations from the two separate calculations and summing them gives a conservative estimate of the total deformation when a real cask hits a real surface. Since the force required to cause a $0.2 \%$ strain $\left(S_{1}\right)$ in the cask is known, the product of this force and the sum of the separately calculated deformations, calculated for the same force, conservatively gives the total deformation energy. By equating this total deformation energy to the kinetic energy, an equivalent velocity can be calculated. This equivalent velocity is then used to modify the curves generated by use of the IMPASC code (in which only an unyielding surface can be modeled) to take into account the effect of the real surface. Figure E-28 shows the analysis for the case of vertical end-drop without limiters. 


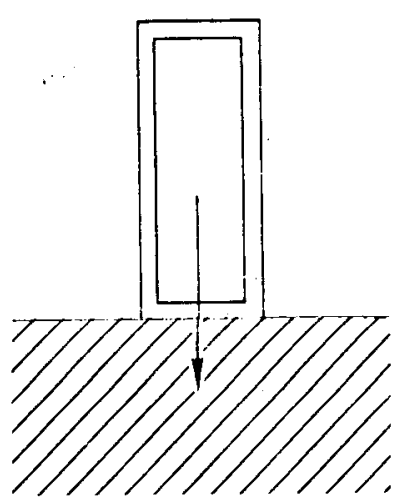

(a) Rigid cask impacts real surface

$\left(\frac{F}{W}\right) g=\frac{V_{1}^{2}}{2 d_{c 1}}$
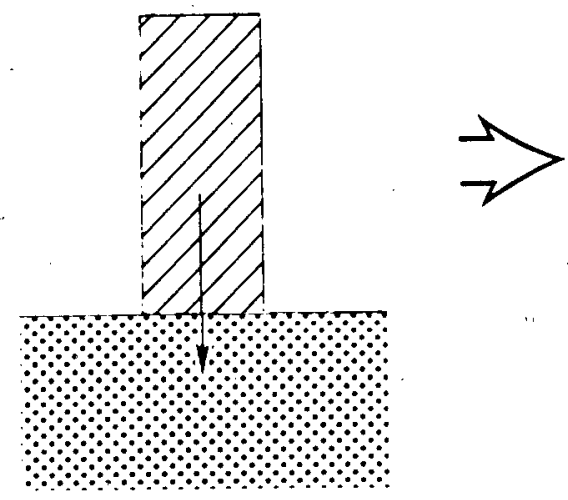

(b) Representative cask impacts unyielding surface

$$
\left(\frac{F}{W}\right) g=\frac{V_{2}^{2}}{2 d_{s 1}}
$$

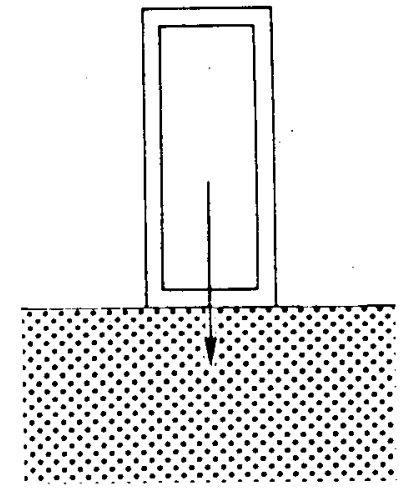

(c) Representative cask impacts real surface

Figure E-28 Equivalent damage technique. 
To illustrate the application of the equivalent damage technique, this discussion is restricted to the case of cask end-drop without limiters and a strain of $0.2 \%\left(\mathrm{~S}_{1}\right)$ even though this technique was used to calculate cask responses for other orientations, for casks with limiters, and higher strain levels.

In case (a), the representative truck or rail cask is impacted onto an unyielding surface so that all the kinetic energy is absorbed by the cask. The strain response of the cask is calculated as a function of impact velocity. Assuming constant deceleration during impact, the deceleration force can be estimated from an energy balance:

$$
\left(\frac{F}{W}\right) g=\frac{v_{1}^{2}}{2 d_{c 1}}=\text { deceleration force in } g \text { for unyielding surface }
$$

where $F$ is the force of impact in pounds, $W$ is the cask weight in pounds, $g$ is the gravitational constant in $\mathrm{ft} / \mathrm{sec}^{2}, V_{1}$ is the impact velocity in $\mathrm{ft} / \mathrm{sec}$, and $d_{c l}$ is the cask deformation in inches.

The cask deformation, $d_{c l}$, is related to the maximum strain on the inner wall where the $0.2 \%$ strain $\left(S_{1}\right)$ level is defined. The deceleration force, cask deformation, and the maximum strain at the inner wall are calculated over a range of impact velocities. The deceleration force, ( $F / W) g$, is identified where the $0.2 \%$ strain $\left(S_{1}\right)$ level occurs.

In case (b), a rigid body with the same outer dimensions as the cask is impacted onto real surfaces such as hard rock, soft rock, and tillable soil. All the kinetic energy is then absorbed by the surface. The deceleration force can be estimated by

$$
\left(\frac{F}{W}\right) g=\frac{v_{2}^{2}}{2 d_{s l}}=\text { deceleration force in of a rigid cask }
$$


where $V_{2}$ is the impact velocity in $\mathrm{ft} / \mathrm{sec}$ and $\mathrm{d}_{\mathrm{sl}}$ is the penetration into the surface in inches Again the deceleration force is calculated over a range of impact velocities. The impact velocity $v_{2}$ is determined for the same impact force identified in case (a) at the $0.2 \%$ strain $\left(S_{1}\right)$ level.

In case (c), the representative cask is impacted onto real surfaces. The impact velocity and kinetic energy are absorbed by both the cask and the surface. The deceleration force can be estimated by

$$
\left(\frac{F}{W}\right) g=\frac{v_{3}^{2}}{2\left(d_{s 1}+\frac{1}{d_{c 1}}\right)}=\begin{aligned}
& \text { deceleration force in } g \text { of a } \\
& \text { representative cask on a real surface }
\end{aligned}
$$

where $V_{3}$ is the impact velocity corresponding to the $0.2 \% \operatorname{strain}\left(S_{1}\right)$ level, and $d_{s l}$ and $d_{c l}$ are the penetration into the surface and cask deformation, respectively, as calculated separately for the same force. By equating Equations E.9 and E.10, the velocity $V_{3}$ is calculated:

$$
v_{3}^{2}=\frac{v_{2}^{2}\left(d_{s 1}+d_{c l}\right)}{\left(d_{s 1}\right)^{-}} \geq v_{2}^{2}
$$

A higher impact velocity is required to give equivalent damage for the case where energy is absorbed by both the cask and the surface. The equivalent damage technique was conservatively applied by assuming that either the cask or the impacted surface absorb all of the impact energy. The resulting average force on the cask was then used to estimate the strain on the inner she11. Consequently, the strain is significantly overestimated in those cases when significant energy is absorbed by both the cask and the surface. As shown by the benchmark calculation, this approach over compensates for the simplifying assumptions made to develop the equivalent damage technique. 
This equivalent damage technique was benchmarked by impacting the representative truck cask on soft rock and then comparing the calculated strain with the estimated strain from the equivalent damage technique for the same impact conditions.

To simplify the comparison, the impact limiter was not included in the benchmark analysis. The representative cask was impacted at $30 \mathrm{mph}$ on the soft rock surface. The cask response to the impact is summarized in Table E.16. The cask response using the equivalent damage technique is also summarized. The percentage strain response for the actual case is $5.4 \%$ compared to $14.3 \%$ estimated using the equivalent damage technique. In this benchmark case, it. was assumed that all of the energy is absorbed by the soft rock, because the resultant force is lower than that resulting from an equivalent drop onto an unyielding surface. From this benchmark calculation it was concluded that the equivalent damage technique as used in this study overestimates the cask response, yet provides reasonable results for estimating purposes.

\section{E.7.2 Soil Impacts}

A simple soil model was developed and benchmarked for evaluating impacts on soil with the representative casks as discussed in Subsections E.7.2.1 and E.7.2.2. The responses of the representative casks for endwise impacts on soil were estimated in Subsection E.7.2.3 using the equivalent damage technique. The responses of the casks were calculated with 2-D cask models in Subsection E.7.2.4 for sidewise impacts.

\section{E.7.2.1 Soil Model}

Three surfaces are considered to represent a range of credible impact scenarios. The surfaces considered simulate a hard rock, a soft rock including concrete, and tillable soil. Real surfaces exhibit complex characteristics but can be considered to deform elastically during the early part of the impact, followed by an energy dissipation phase. The exact nature of the energy dissipation mechanisms is not well known; therefore, a reasonable and simple elastic-perfectly plastic formulation was used. The two 
Table E.16

Comparison of Equivalent Damage Technique Result with Real Surface Impact Results

\begin{tabular}{lcccc}
\hline & $\begin{array}{c}\text { Real Cask } \\
\text { on Soft } \\
\text { Rock } \\
\text { Surface }\end{array}$ & $\begin{array}{c}\text { Rigid Cask } \\
\text { on Soft } \\
\text { Rock } \\
\text { Surface }\end{array}$ & $\begin{array}{c}\text { Real Cask on } \\
\text { Unyielding } \\
\text { Surface }\end{array}$ & $\begin{array}{c}\text { Equivalent } \\
\text { Damage } \\
\text { Technique }\end{array}$ \\
\hline Cask Velocity (mph) & 30 & 30 & 28.4 & 30.0 \\
Duration of Impact (msecs) & 17 & 7.5 & 17.0 & 17.0 \\
Interface Force at Impact (g) & 203 & 222 & 222.0 & 222.0 \\
Maximum Plastic Strain (\%) & 5.4 & N/A & 14.3 & 14.3 \\
Lead Slump (in) & 6 & N/A & 6.12 & 6.12 \\
\hline
\end{tabular}


parameters used in this formulation, namely the initial elastic modulus and the yield stress, can be calibrated to approximate an equivalent energyabsorbing medium. To provide the calibration, penetration data E.19 were used as discussed in Subsection E.7.2.2. Reasonable predictions of penetration were possible using the equivalent elastic-plastic formulation.

The material parameters required by the bilinear computer model, an elastic-plastic model referred to as Material Type 3 in the NIKE/DYNA input manua 1s, are

$$
\begin{aligned}
& E=\text { Young's modulus, psi, } \\
& \nu=\text { Poisson's ratio, unitless, } \\
& \sigma_{y}=\text { yield strength, psi, } \\
& \rho=\text { density, } 1 \mathrm{~b}-\sec ^{2} / \mathrm{in}^{4}, \\
& \beta=\text { hardening parameter, unit less, } \\
& E_{n}=\text { hardening modulus, psi, }
\end{aligned}
$$

$\sigma_{1 u l t}$ and $\sigma_{3 u 1 t}=$ principle stresses at ultimate stress state, psi.

A suitable range of yield stresses had to be determined for use within the elastic-perfectly plastic model. The standard method for predicting soil failure is the Mohr-Coulomb failure criterion, which states that soil will fail in shear at a value proportionat to the applied confining pressure, which varies with soil depth. "Even if it is asumed that yielding begins at a stress level equal to the failure stress (corresponding to the elasticperfectly plastic response assumption), it is necessary to consider a range of failure stress levels. E.17

To calculate the failure stress, oult, the data of J.M. Duncan, et a1., were used to provide an extensive list of soil parameters. E.18 Also, a 
relationship between the deviatoric failure stress, $\left(\sigma_{1}-\sigma_{3}\right)_{f}$, the friction angle, $\theta$, and the cohesion intercept is given by Duncan with the formula

$$
\left(\sigma_{1}-\sigma_{3}\right)_{f}=2 c \cos \theta+2 \sigma_{3} \sin \theta .
$$

The deviatoric failure stress is related to the ultimate deviatoric stress as follows: E. 17

$$
\left(\sigma_{1}-\sigma_{3}\right)_{f}=R_{f}\left(\sigma_{1}-\sigma_{3}\right)_{u 1 t}
$$

where $R_{f}$ is the failure ratio. Because $\left(\sigma_{1}-\sigma_{3}\right)_{f}$ is always less than $\left(\sigma_{1}-\sigma_{3}\right)_{u l t}$, the value of $R_{f}$ is always less than 1 , usually 0.5-0.9. Duncan lists soil parameters for about 150 soils. If, for a particular type of soil, e.g. sandy clay, the largest $\theta, c$, and $\sigma_{3}$, and the lowest value for $R_{f}$ are selected, a conservative value for the deviatoric failure stress can be calculated. Rearranging equation (E.13) gives:

$$
\sigma_{u 1 t}=\sigma_{1 u 1 t}=-\frac{\left(\sigma_{1}-\sigma_{3}\right)}{R_{f}}+\sigma_{3 u 1 t} .
$$

For an elastic-plastic model it is conservative to use the ultimate stress as the yield stress to estimate the maximum force on the cask.

From Duncan's data a summary of the conservative parameters found for 12 general categories of soils is given in Table E.17. 
Table E.17

Soil Parameters

\begin{tabular}{lccccr}
\hline \multicolumn{1}{c}{ Soils } & $\begin{array}{c}\text { Max } \\
\left(^{\circ}\right)\end{array}$ & $\begin{array}{c}\text { Max } c \\
\left.\text { (tons/ft }{ }^{2}\right)\end{array}$ & $\begin{array}{c}\text { Max } \\
\text { (psi) }\end{array}$ & $\begin{array}{c}\sigma_{3} \\
\text { (unitless) }\end{array}$ & $\begin{array}{c}\sigma_{y} \\
\text { (psi) }\end{array}$ \\
\hline Rockfill & 53 & 0 & 728 & 0.51 & 12051 \\
Sandy Gravel & 58 & 10.01 & 728 & 0.57 & 15107 \\
Clayey Grave1/Clayey Sand & 34 & 2.6 & 504 & 0.55 & 2847 \\
Silty Sand/Sandy Silt & 53 & 0.54 & 219 & 0.57 & 3277 \\
Sand & 49 & 0 & 1104 & 0.63 & 11892 \\
Silty Clay & 33 & 3.3 & 222 & 0.58 & 1161 \\
Lean Clay & 3 & 1.10 & 93.33 & 0.52 & 118 \\
Fat Clay & 4 & 1.5 & 156 & 0.65 & 196 \\
Silt & 45 & 0 & 115 & 0.57 & 1090 \\
& & & & & \\
\hline
\end{tabular}




\section{E.7.2.2 Soil Model Benchmark Calculations}

The soil model was benchmarked by comparing with test data. This was accomplished in two phases. The first was an analytical plate bearing test. This test is offten used to evaluate soils., subgrades, and pavements, especiality in road design, and uses the modulus of subgrade reaction, $k$, which is measured in situ with a plate bearing test. The test involves loading a circular disk, or stack of disks, usually 30 inches in diameter, at a specifijed deflection rate, and measuring the deflection at a predetermined load, usually 10 psi. The modulus $k$ is calculated as follows:

where

$$
k=\frac{p}{\Delta}, p s i / i n,
$$

$$
\begin{aligned}
& p=\text { unit load on plate, psi } \\
& \Delta=\text { deflection of plate, inches. }
\end{aligned}
$$

The results obtained for the modulus of subgrade reaction, $k$, were compared with predicted values $\mathrm{E}^{\mathrm{.} .18}$ and are summarized in Table E.18. The purpose of this check was to verify that the selected elastic plastic material model produced result.s that were not completely out of 1 ine. The results indicate that for elastic loads, the model significantly over-predicts the soil stiffness. The over-prediction is conservative for this study.

The second phase of the benchmark process was a review of work presented by C.W. Young,, E.20 and a comparison of his results with the soil model results. Young's method was developed to predict depth of earth-penetrating projectiles. Young uses a material parameter, which he calls 'S, in his formulation and has tabulated values of ' $S$ for a large variety of soils. Typical values of $S$ from Young E..20 are listed in Table E.19 with the bilinear soil parameters. E.17, E.. 19, E.21

From Table E.18 it is concluded that the parameters used to model soits can vary over a wide range for different types of soil and rocks. Also the types of soills and rocks can vary significantly within a specific land region.. To make the work manageable in analyzing impact with surfaces, the 
Table E.18

Plate Bearing Test Simulation with NIKE 2-D

\begin{tabular}{|c|c|c|}
\hline Soil & $\begin{array}{c}\text { Calculated } k \\
(p s i / i n)\end{array}$ & $\begin{array}{c}\text { Predicted kE.19 } \\
\text { (psi/in) }\end{array}$ \\
\hline 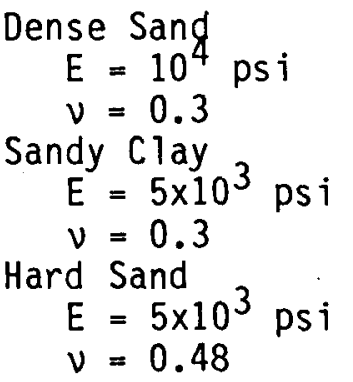 & 1100 & 300 or more \\
\hline
\end{tabular}


Table E.19

Summary of Soil Types and Range of Soil Parameters E.17,E.19, E.21

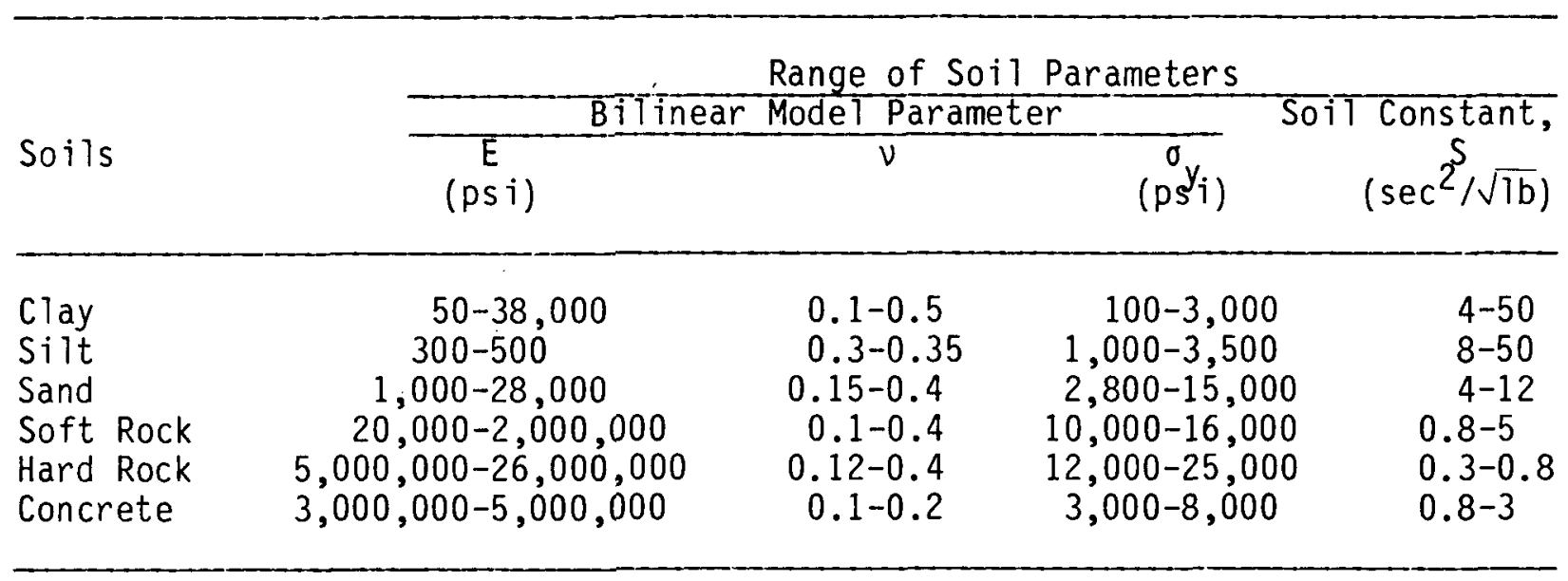


surfaces were classified into three groups: hard rock, soft rock including concrete, and tillable soil. The material properties selected to represent each of these groups are tabulated in Table E.20. The range of values for the parameters and Young's E.20 soil constant $S$ are tabulated for each group.

In Fig. E-29, the impact forces calculated using the elastic plastic model are plotted for impact on each of the three surfaces by a rigid truck cask as a function of impact velocity. Impact force ranges calculated using experimental formulas ${ }^{E .20}$ and a rigid truck cask are also plotted for general $S$ soil constants for comparison. For each of the groups there is good agreement between the DYNA 2-D results and the experimental ones.

\section{E.7.2.3 Endwise Impacts on Soit}

In order to use the equivalent damage technique to estimate the response of the representative casks for endwise impacts on real surfaces, the impact forces had to be calculated. These analyses were 2-D axisymmetric dynamic finite element analyses, using the code DYNA 2-D. A typical mesh is shown in Fig. E-30. The model includes an unyielding cylindrical falling body which has the same weight and radius as the representative truck and rail casks. A slideline was included between the unyielding cask and the surface. Slideline type three, sliding with voids, was selected from the DYNA 2-D Users Manual. The other possibility, slideline type four, was rejected because the penalty formulation required some adjustment depending upon the stiffness of the soil and the impact velocity, which was not suitable for a parametric study. The impact forces were calculated at four velocities, 30, 60, 90, and $120 \mathrm{mph}$. The impact forces are summarized in Table E.21 for the representative truck and rail casks.

\section{E.7.2.4 Sidewise Impacts on Soil}

Two-dimensional plane strain analys is without impact limiters or end closures were performed for sidewise impacts on the three surfaces to estimate the 3-D responses of the two representative casks. The 2-D truck and rail cask models were developed using the MAZE interactive mesh generators. The cask models do not include contents. DYNA 2-D was used to calculate the responses. 
Table E.20

Selected Soil Parameters for this Study

\begin{tabular}{|c|c|c|c|c|}
\hline $\begin{array}{l}\text { Represented } \\
\text { Surface }\end{array}$ & $\frac{\text { Bilinear }}{E}$ & $\frac{\text { Mode } 1}{v}$ & $\frac{\text { Parameter }}{\sigma_{y_{i}}}$ & $\begin{array}{c}\text { Soil Constant, } S \\
\text { Range } \\
\left(\sec ^{2} / \sqrt{1 b}\right)\end{array}$ \\
\hline $\begin{array}{l}\text { Soi } 1 \\
\text { Soft Rock, } \\
\text { Concrete } \\
\text { Hard Rock }\end{array}$ & $\begin{array}{r}6,000 \\
3,640,000 \\
7,000,000\end{array}$ & $\begin{array}{l}0.4 \\
0.2\end{array}$ & $\begin{array}{r}1,000 \\
4,000 \\
25,000\end{array}$ & $\begin{array}{l}5-50 \\
1-5 \\
0.3\end{array}$ \\
\hline
\end{tabular}




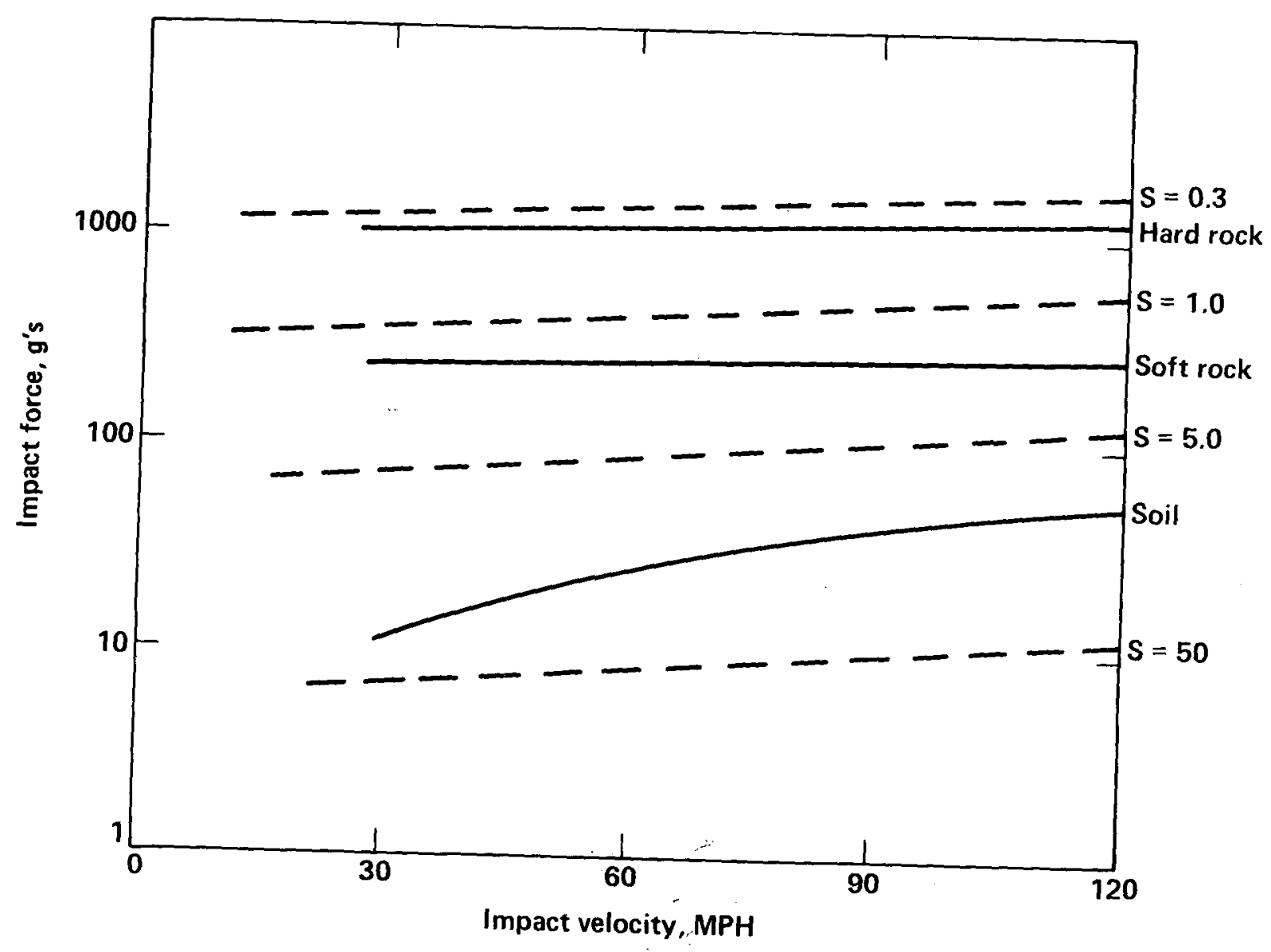

$S=$ Soil constant

Figure E-29 Soil model comparison with penetration test data. 


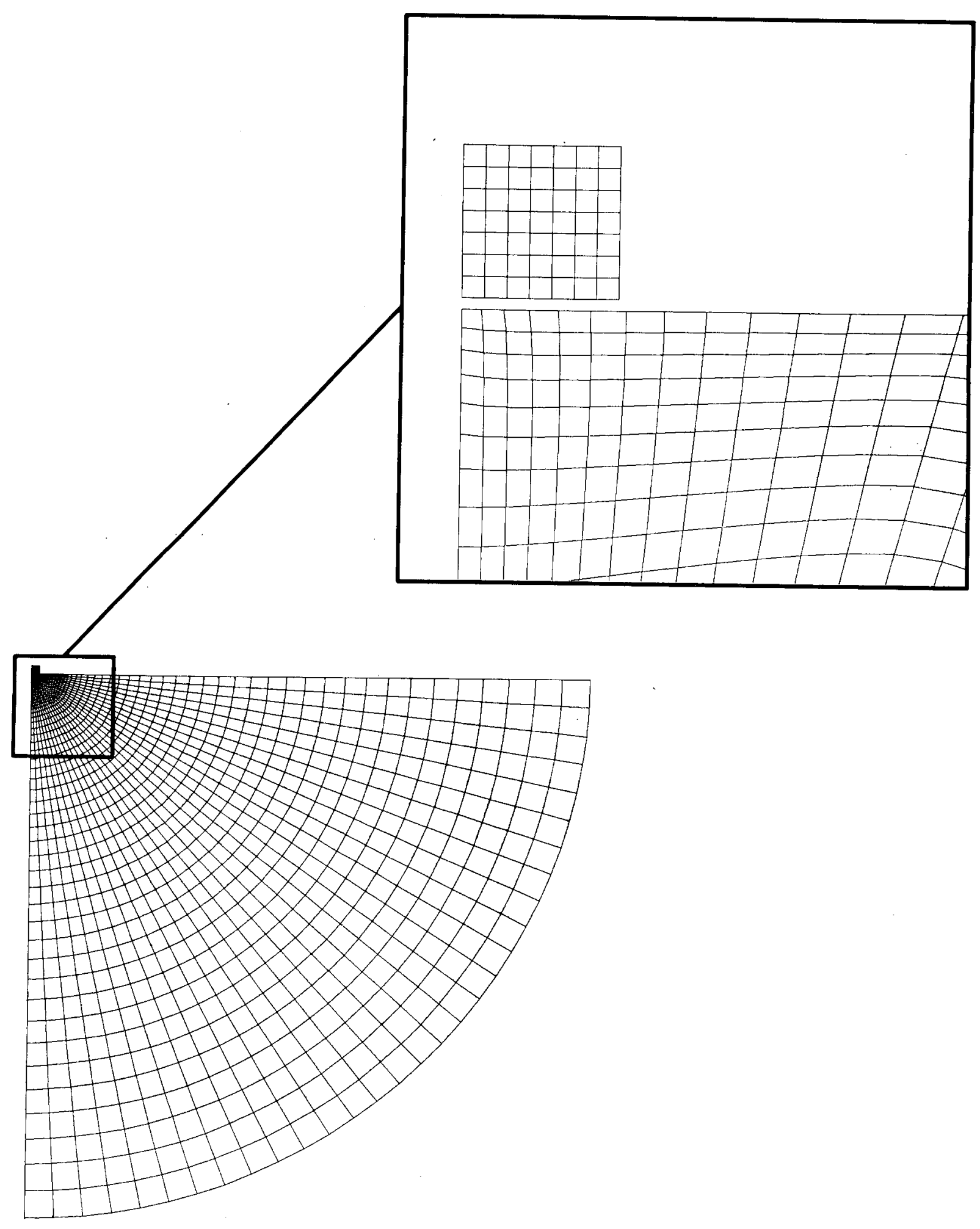

Figure E-30 Finite element mesh for drops on soils. 
Table E.21

Summary of Cask Responses to Endwise Impacts on Real Surfaces

\begin{tabular}{|c|c|c|c|c|c|c|}
\hline \multirow[b]{4}{*}{$\begin{array}{c}\text { Velocity } \\
\text { (mph) }\end{array}$} & \multicolumn{6}{|c|}{$\begin{array}{c}\text { Impact Force } \\
(\mathrm{g})\end{array}$} \\
\hline & \multicolumn{3}{|c|}{ Truck Cask } & \multicolumn{3}{|c|}{ RajT Cask } \\
\hline & \multicolumn{3}{|c|}{ Surface Type } & \multicolumn{3}{|c|}{ Surface Type } \\
\hline & $\begin{array}{l}\text { Hard } \\
\text { Rock }\end{array}$ & $\begin{array}{l}\text { Soft } \\
\text { Rock }\end{array}$ & So il & $\begin{array}{l}\text { Hard } \\
\text { Rock }\end{array}$ & $\begin{array}{l}\text { Soft } \\
\text { Rock }\end{array}$ & Soil \\
\hline 30 & 1050 & 250 & 12 & -- & 420 & 16 \\
\hline 60 & 1310 & 270 & 26 & -- & -- & 110 \\
\hline 90 & 1340 & - & 40 & -- & 600 & 200 \\
\hline 120 & 1360 & 290 & 54 & -- & -- & -- \\
\hline
\end{tabular}




\section{E.7.2.4.1 Truck Cask Impacts}

The truck cask response to endwise impacts on hard rock surfaces was essentially the same as the response to impact on an unyielding surface. Since the cask stiffness is less for sidewise impacts, sidewise impact analyses were performed only for impacts on soil and soft rock. The calculations were performed for $30 \mathrm{mph}$ and $60 \mathrm{mph}$ impacts on soil and $30 \mathrm{mph}$ and 90. mph impacts on soft rock. The effective plastic strain distribution at the time the maximum occurs is shown in Fig. E-31 for the $60 \mathrm{mph}$ impact on soil. The results of the impact calculations are summarized in Table E.22. The maximum strain response of the cask was $2.45 \%$ and $7.62 \%$ at the inner she 11 for the $30 \mathrm{mph}$ and $60 \mathrm{mph}$ impacts on the soil. The strain response at the inner she 11 was $5.03 \%$ and $13.6 \%$ for the impacts on the concrete surface at $30 \mathrm{mph}$ and $90 \mathrm{mph}$, respectively.

\section{E.7.2.4.2 Rail Cask Impacts}

As was done for the truck cask, sidewise impact analyses were performed for the rail cask for impacts on soil and soft rock. The calculations were performed for $30 \mathrm{mph}$ and $60 \mathrm{mph}$ impacts on soil and $30 \mathrm{mph}$ and 90 mph impacts on soft rock. The effective plastic strain distribution at the time the maximum occurs is shown in Fig. E-32 for one of the cases studied. The results of the impact calculations are summarized in Table E.23. The maximum strain responses at the inner she 11 for impacts on soil were $2.17 \%$ and $3.37 \%$ at $30 \mathrm{mph}$ and $60 \mathrm{mph}$, respectively. The maximum strain responses of the rail cask was lower than those of the truck cask because of its greater flexibility.

\section{E.7.3 Water Impact}

An analys is of water impact for wedge shaped bodies is provided in the literature for use in ship hull design. E.22,E.23 A phenomenon, substantiated during an experimental investigation of flat bottom slamming at the Naval Ship Research and Development Center, is described wherein, during flat bottom slamming, air is trapped between the impact surface of the falling body and the water surface, thereby cushioning the impact. E.23 Thus the impact angle 


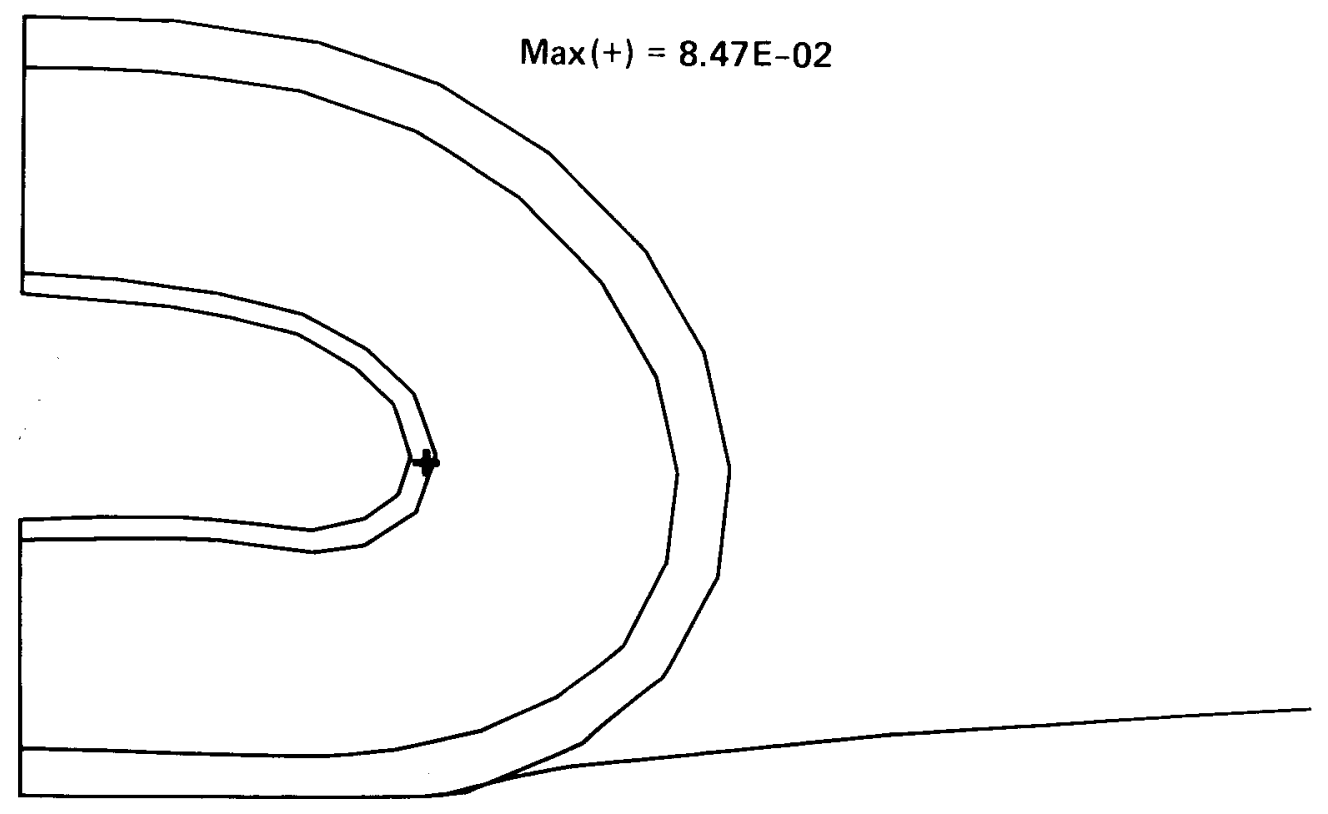

Figure E-31 Maximum plastic strain location on truck cask for impact at 60 mph on soft rock. 
Table E.22

Results of Truck Cask Sidewise Impacts on Real Surfaces (without Impact Limiter)

\begin{tabular}{|c|c|c|}
\hline \multirow[b]{3}{*}{$\begin{array}{l}\text { Velocity } \\
\text { (mph) }\end{array}$} & \multicolumn{2}{|c|}{$\begin{array}{c}\text { Strain at Inner Wall } \\
(\%)\end{array}$} \\
\hline & \multicolumn{2}{|c|}{ Surface Type } \\
\hline & Soil & Soft Rock \\
\hline $\begin{array}{l}30 \\
60 \\
90\end{array}$ & $\begin{array}{l}2.45 \\
7.62 \\
--\end{array}$ & $\begin{array}{l}5.03 \\
-- \\
13.6\end{array}$ \\
\hline
\end{tabular}




$$
\operatorname{Min}(-)=0
$$

$\operatorname{Max}(+)=3.37 E-02$

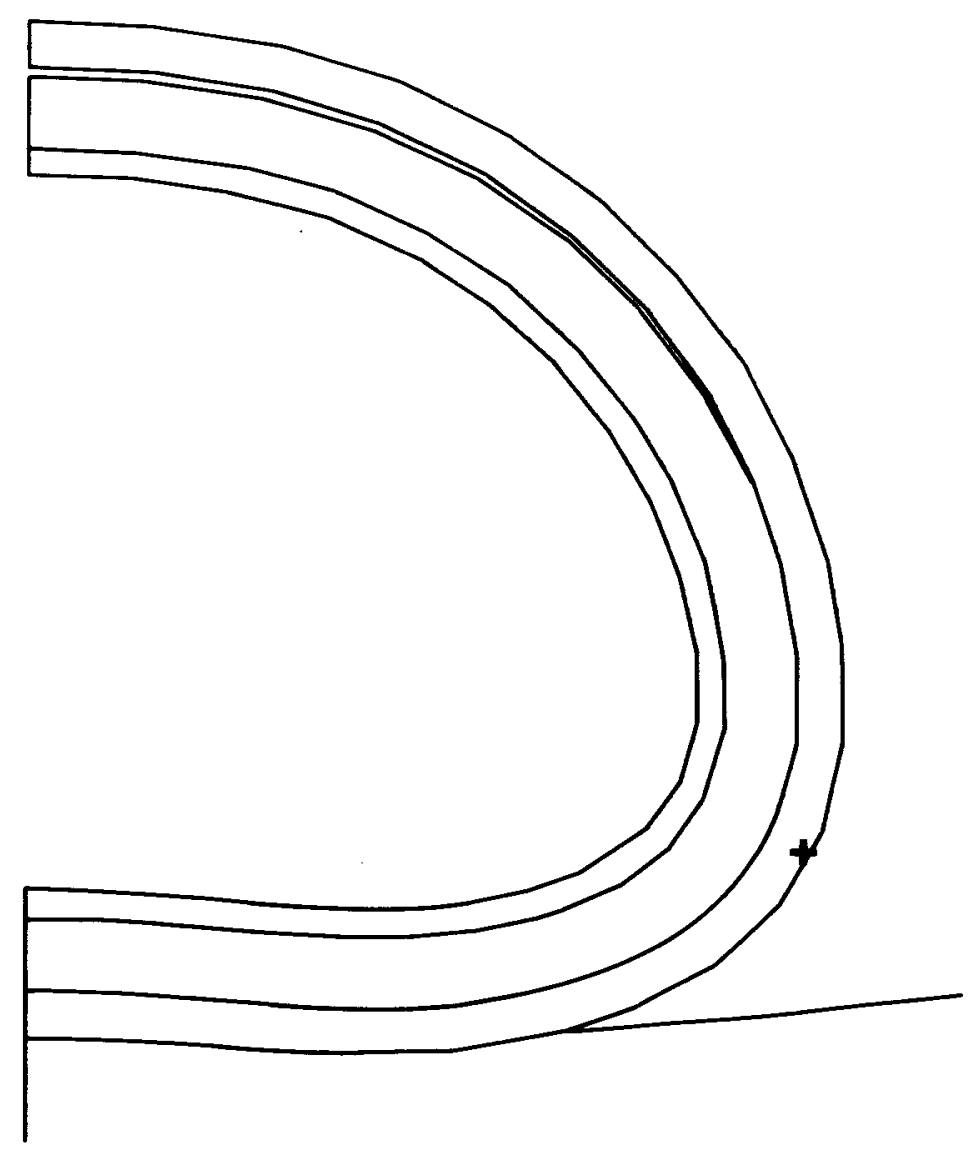

Figure E-32 Maximum plastic strain location on rail cask for impact at 60 mph on soft rock. 
Table E.23

Results of Rail Cask Sidewise Impacts on Real Surfaces (without Impact Limiter)

\begin{tabular}{ccc} 
& \multicolumn{2}{c}{$\begin{array}{c}\text { Strain at Inner Wall } \\
(\%)\end{array}$} \\
\cline { 2 - 3 } $\begin{array}{c}\text { Velocity } \\
(\mathrm{mph})\end{array}$ & \multicolumn{1}{c}{ Surface Type } \\
\cline { 2 - 3 } & Soit & Soft Rock \\
60 & 2.17 & 3.78 \\
90 & 3.37 & 10.10 \\
\hline
\end{tabular}


producing the highest impact force is not $90^{\circ}$, but $87^{\circ}$. An approximation of the impact force on a cask falling into a body of water is made by integrating the pressure, over an area equal to the cask end cross sectional area: E.23

$$
p(x)=\frac{1}{2} \rho V^{2}\left[\frac{\pi}{(90-\beta)\left(1-\frac{x^{2}}{L^{2}}\right)^{1 / 2}}-\frac{\frac{x^{2}}{L^{2}}}{1-\frac{x^{2}}{L^{2}}}+\frac{2 \pi}{V^{2}}\left(L^{2}-x^{2}\right)^{1 / 2}\right]
$$

and

$$
\text { Force }=\int_{0}^{L} q p(y) d y
$$

where

$$
\begin{aligned}
& y=L x, f t, \\
& q=2 L\left(x-x^{2}\right)^{1 / 2}, f t, \\
& B=\text { compliment of deadrise angle, }{ }^{0}, \\
& \rho=\text { mass density of water, } \mathrm{lb} / \mathrm{ft}^{3}, \\
& L=\text { cask diameter, ft, } \\
& V=\text { cask impact velocity, ft/sec, } \\
& \ddot{z}=0 \text { (i.e., assume that impact acceleration, = } \mathrm{g} \text {, is }
\end{aligned}
$$


Using Simpson's Rule for integration, the interface forces were calculated for the two unyielding casks with the same external dimensions as the representative casks for four impact velocities and three cask orientations. For the large diameter rail cask, the loads due to impact on water can be quite wigh for the $87^{\circ}$ impact angle. However these loads drop off rapidly for other impact angles. The results of the calculations are summarized in Table E..24. The equivalent damage technique is used to estimate the strain response of the casks to the calculated impact forces.

\section{E.7.4 Train Sill Impacts}

\section{E.7.4.1 Impact on Truck Cask}

Two scenarios were evaluated for a locomotive sill impacting a truck cask: the sill impacting the cask sidewise head-on; and the sill impacting the cask sidewise off-center. The cross-section of the model used to simulate a locomotive sill is shown in Fig. E-33 and consists of two plates connected with two large I-beams. E.9 The sill was modeled as a solid object with modified properties. For the sidewise head-on impacts the sill was modeled as a plate 3.5 inches thick to approximate its axial strength. For the sidewise off-center impacts the sill was modeled as a plate 11.5 inches thick to approximate its bending strength. In both cases, the density of the sill was calculated for a locomotive weight of 200 tons.'

The si77 was first modeled as shown in Fig. E-34 to impact at a point at $45^{\circ}$ on the truck cask from the sidewise head-on position. Calculations were made with the locomotive sill impacting the cask at velocities of $30 \mathrm{mph}$ and $60 \mathrm{mph}$. In both cases, the cask moved away from the sill at an angle and achieved a velocity lower than the, initial sill velocity. Also, the sill underwent a slight rotation and remained undamaged. However, the cask sustained large deformations where the sill scraped across it. Also as illustrated in Fig. E-35., the sudden acceleration caused the cask to flatten.

For the $30 \mathrm{mph}$ impact, the cask experienced a force of $110 \mathrm{~g}$ 's and sustained a maximum effective stress of 40,100 ps $i$ and maximum plastic strain of $7.5 \%$ in the steel shel1.s. For $60 \mathrm{mph}$, the force increased to $206 \mathrm{~g}$ 's, the 
Table E.24

Interface Force for Water Impact

(A11 Results Listed in Multiples of Cask Weight, No Impact Limiters or Cab Crush Included)

\begin{tabular}{|c|c|c|c|c|c|c|}
\hline \multirow[b]{3}{*}{$\begin{array}{l}\text { Velocity } \\
\text { (mph) }\end{array}$} & \multirow{2}{*}{\multicolumn{3}{|c|}{$\frac{\text { Unyielding Truck Cask }}{\text { Impact Orientation }}$}} & \multirow{2}{*}{\multicolumn{3}{|c|}{$\frac{\text { Unyielding Rail Cask }}{\text { Impact Orientation }}$}} \\
\hline & & & & & & \\
\hline & $87^{\circ}$ & $45^{\circ}$ & $0^{0}$ & $87^{\circ}$ & $45^{\circ}$ & $0^{\circ}$ \\
\hline $\begin{array}{r}30 \\
60 \\
90 \\
120\end{array}$ & $\begin{array}{r}17.7 \\
70.8 \\
159.3 \\
283.2\end{array}$ & $\begin{array}{r}0.9 \\
3.6 \\
8.5 \\
14.5\end{array}$ & $\begin{array}{l}12.6 \\
50.4 \\
119 \\
203\end{array}$ & $\begin{array}{r}37.8 \\
151.3 \\
340.5 \\
605.3\end{array}$ & $\begin{array}{r}1.9 \\
7.6 \\
17.1 \\
30.4\end{array}$ & $\begin{array}{r}10 \\
39 \\
88 \\
155\end{array}$ \\
\hline
\end{tabular}




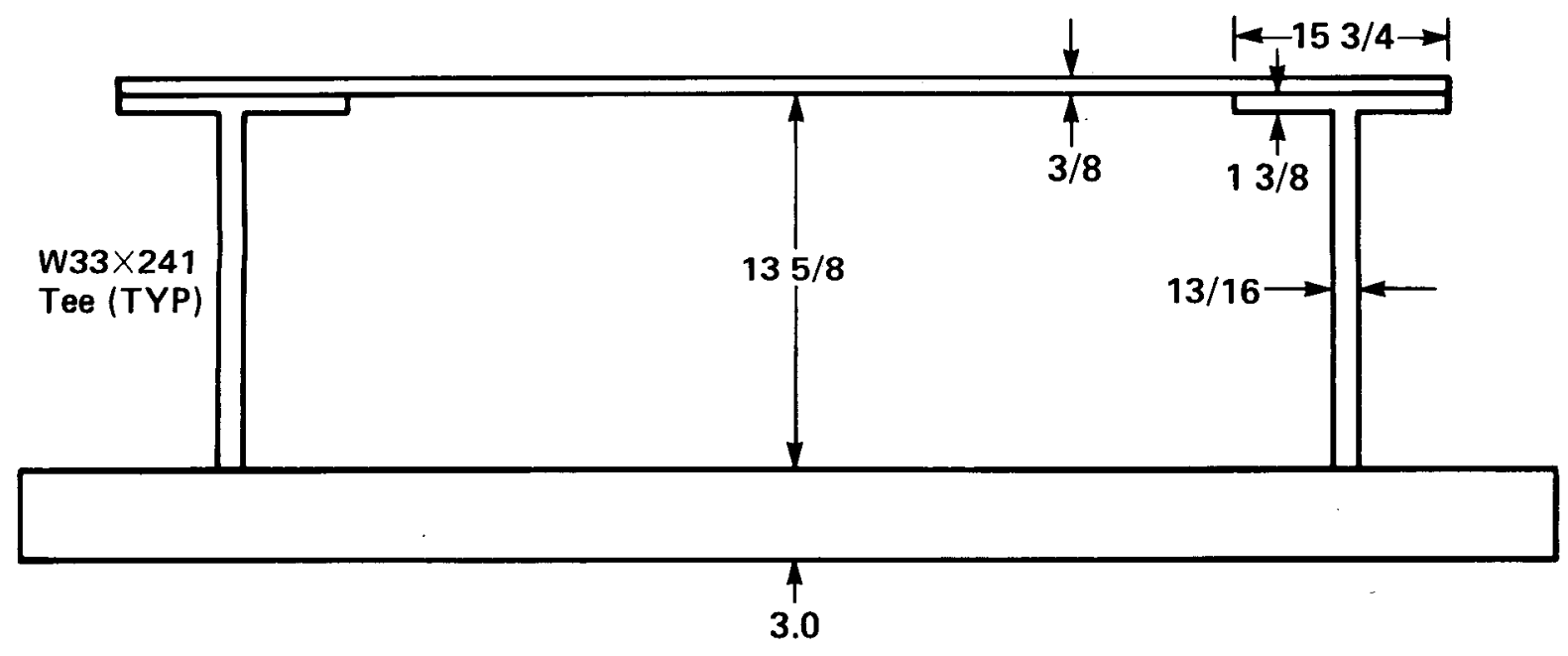

Dimensions in inches

Figure E-33 Locomotive sill cross section. 


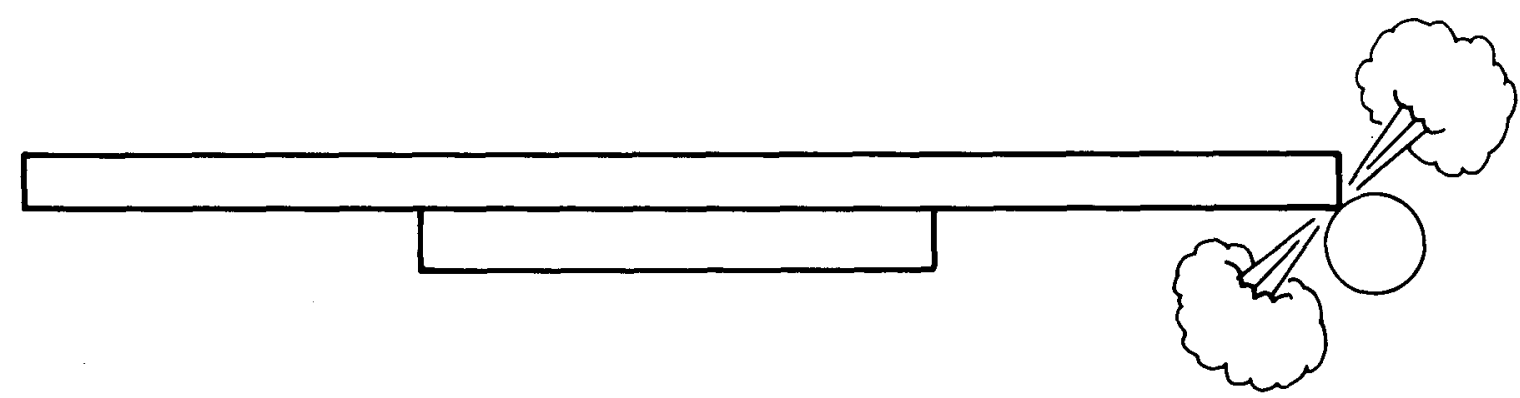

Figure E-34 Sidewise off-center locomotive sill impact.

$$
E-87
$$



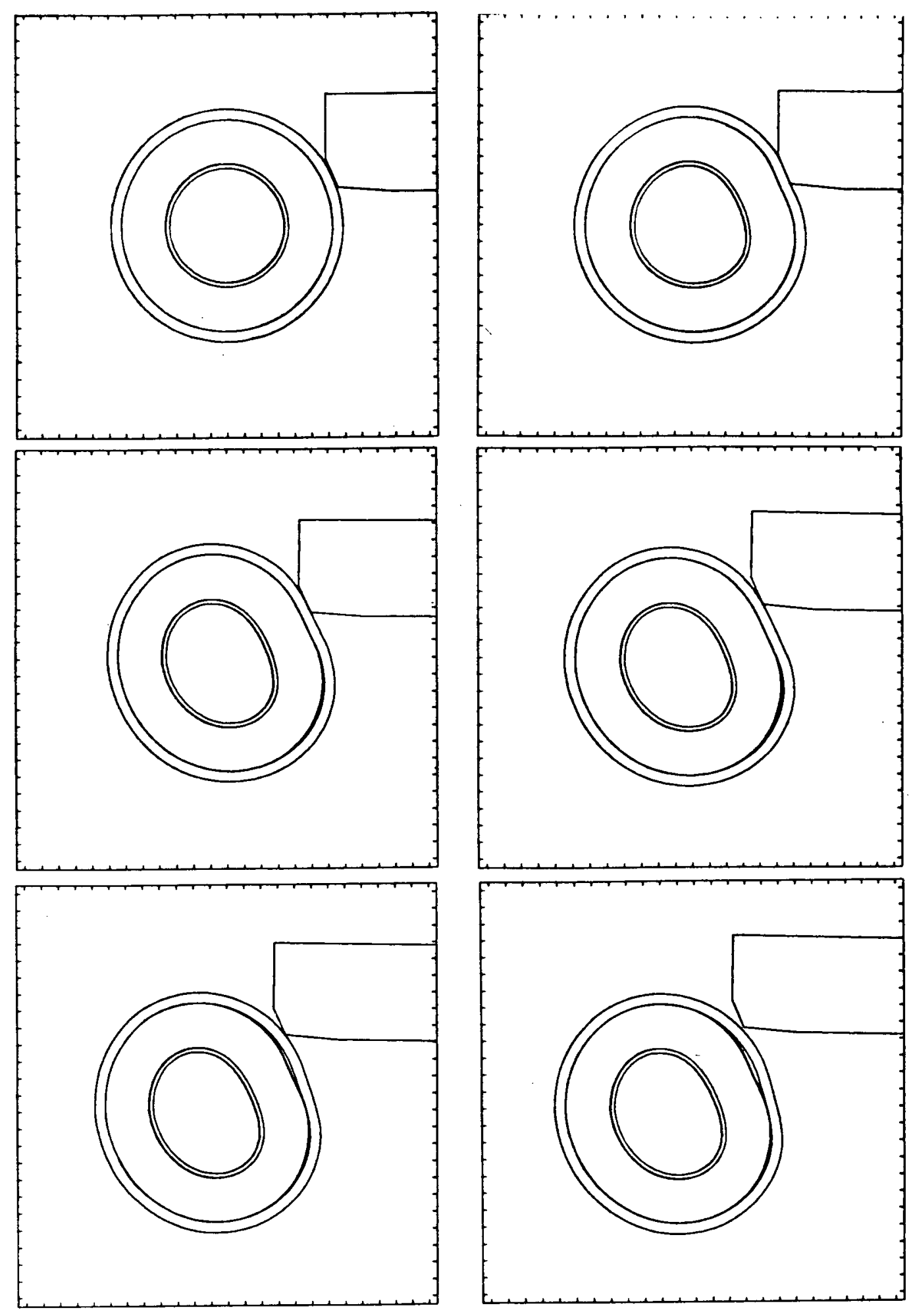

Figure E-35. Thirty mph sidewise off-center sill impact. 
maximum effective stress increased to 50,000 psi, and the maximum plastic strain increased to $12.8 \%$. These results are summarized in Table E.25. The location of the maximum plastic strain is shown in Fig. E-36.

For the sidewise head-on impact, the complete 2-D strain truck cask model was analyzed for impact with the locomotive sill. This model was used only for the impact at $30 \mathrm{mph}$; based on these results, symmetry was used for the 60 mph impact to reduce the solution cost. The modeling is shown in Fig. E-37. In both cases, the cask achieved a velocity higher than the sill velocity and the sill was undamaged. However, the cask sustained large deformations in the impact zone. Also, the sudden acceleration caused the cask to flatten as shown in Fig. E-38.

For the $30 \mathrm{mph}$ impact, the cask experienced a force of $138 \mathrm{~g}$ 's, a maximum effective stress of $50,000 \mathrm{psi}$, and a maximum plastic strain of $12.4 \%$ in the steel shells. For $60 \mathrm{mph}$, the force increased to $265 \mathrm{~g}$ 's, the maximum effective stress increased to 65,000 psi, and the maximum plastic strain increased to $20 \%$. These results are summarized in Table E.26. The location of the maximum plastic strain is shown in Fig. E-39.

None of our cask models included contents. For the truck cask, the mass of the contents is not large compared to the mass of the cask. The truck cask is very much like a thick-walled cylinder and under the severe impact conditions, it is able to support itself. For the rail cask, the mass of the contents is very large compared to the mass of the cask. Also, the rail cask is like a thin-walled cylinder. Under the severe impact conditions, it is unable to support itself. Thus, contents are very important to the rail cask calculations and should be modeled to provide more accurate impact forces and $\mathrm{g}$ loads and to support the cask as it collapses.

Our comparison of the maximum effective stresses and plastic strains sustained by the two casks for the different impact conditions shows that the sidewise sill head-on impact into the truck cask is the most severe. The offcenter impact is less severe because the sill transfers less energy as it strikes a glancing blow to the cask. The truck cask impacting on the unyielding surface is less severe than the sidewise head-on impact. However, the maximum $\mathrm{g}$ loads occur in the impacts on an unyielding surface. The E-89 
Table E.25

Results Sidewise of Off-Center Sill Impact Against Truck Cask

Locomotive sill velocity (mph)

Locomotive sill velocity (in/sec)

Duration of impact (sec)

Velocity at end of impact (in/sec)

Angle of departure of cask $\left({ }^{\circ}\right)$

$\mathrm{g}$ load on cask $(\mathrm{g})$

Maximum effective stress (psi)

Maximum plastic strain (\%)

Maximum plastic strain at inner shell(\%)
$30 \quad 60$

$528 \quad 1056$

$0.012 \quad 0.011$

$425 \quad 637$

52

110

40,100

7.5

2.3
42

206

50,800

12.8 
$\operatorname{Max}(+)=7.54 \mathrm{E}-02$

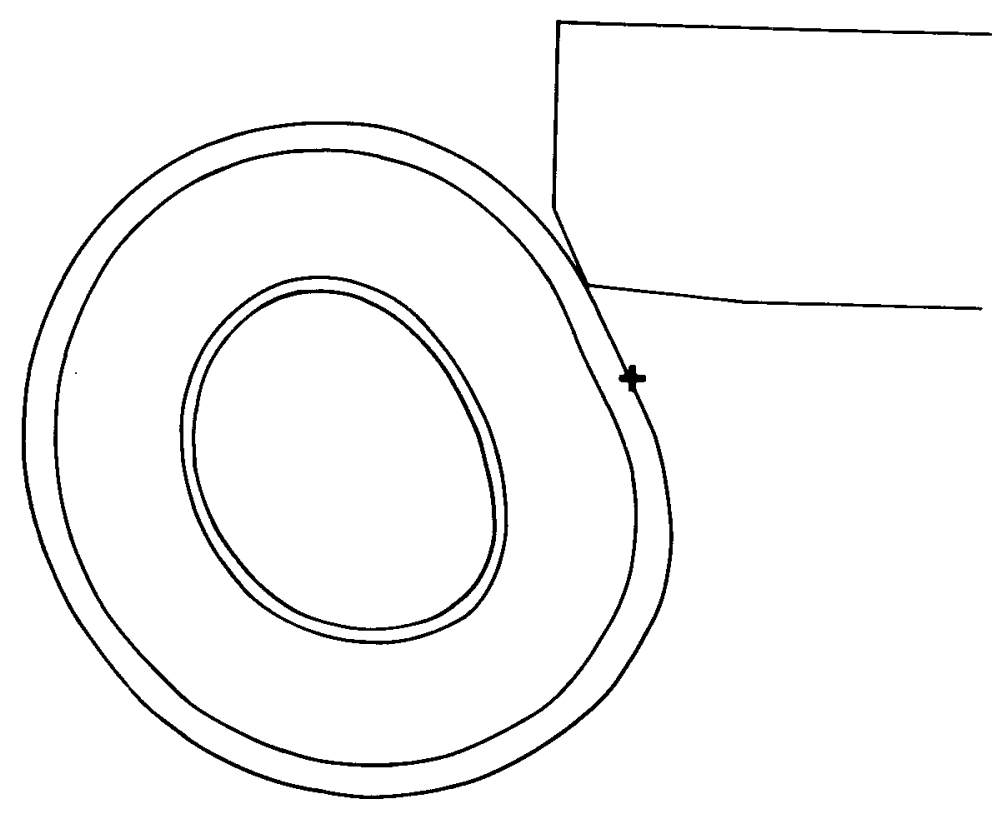

Figure E-36 Thirty mph:sidewise off-center sill impact-maximum plastic strain location. 


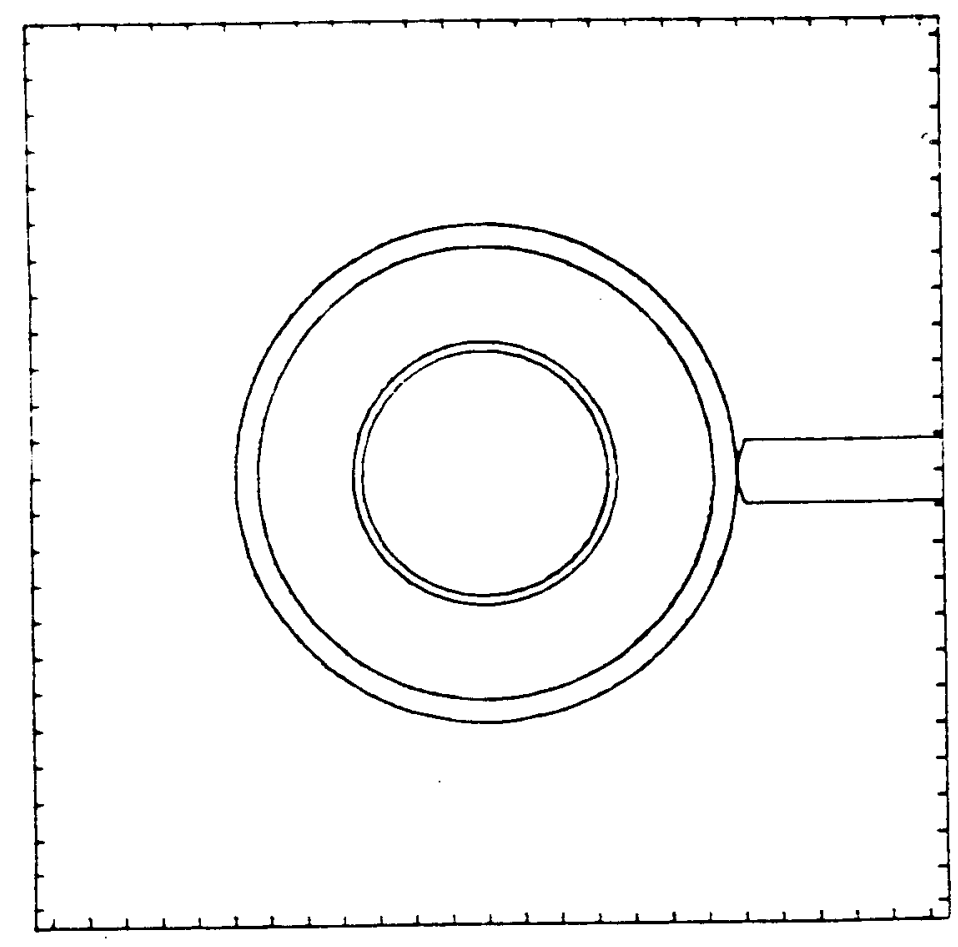

$30 \mathrm{mph}$ model

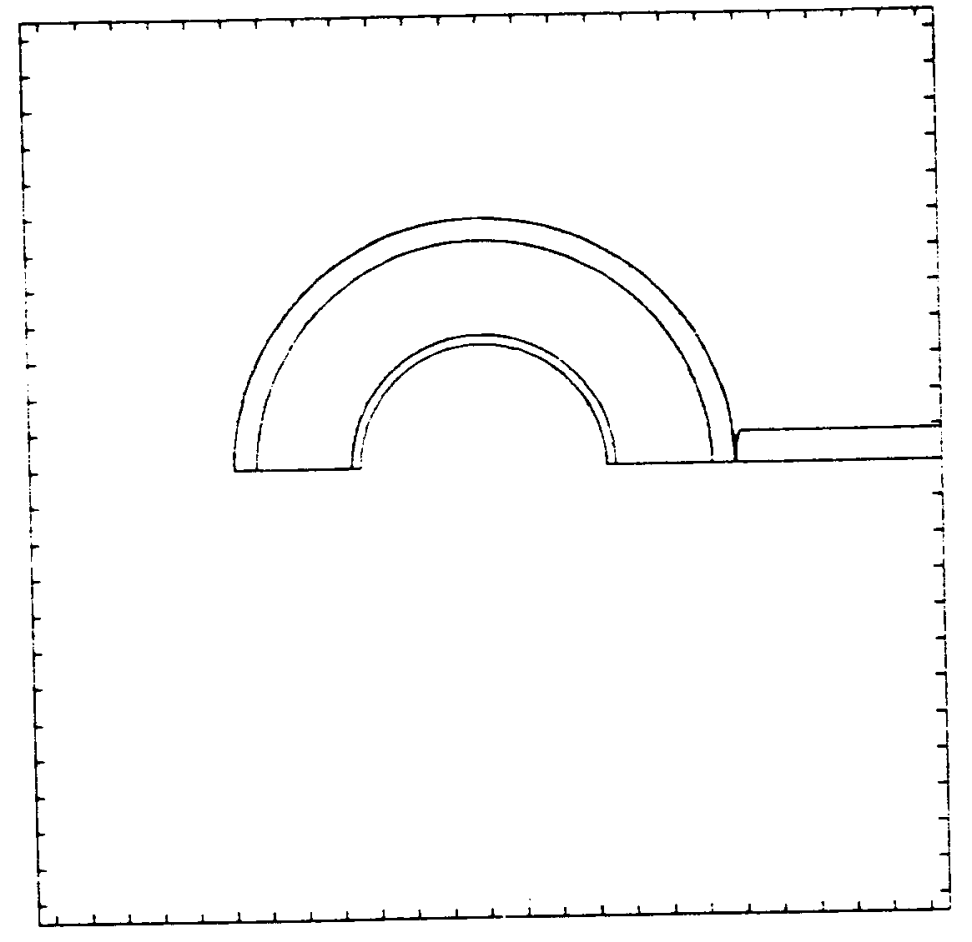

$60 \mathrm{mph}$ model

Figure E-37 Mode1 configurations for sidewise head-on sill impact. Note use of symmetry for $60 \mathrm{mph}$ case. 

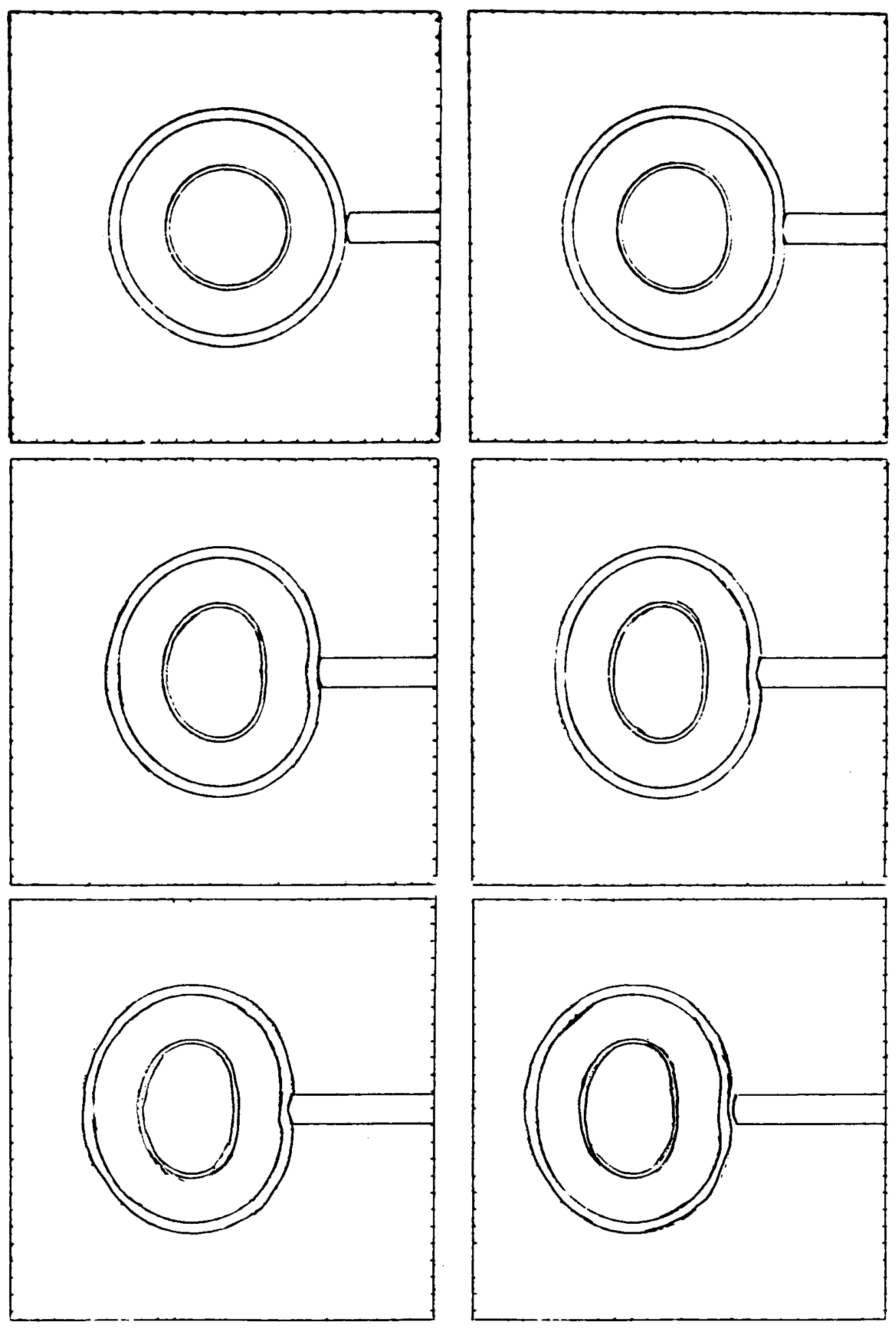

Figure E-38 Sidewise head-on sill impact at $30 \mathrm{mph}$. 
Table E.26

Results of Sidewise Head-on Sill Impact Against Truck Cask

\begin{tabular}{lrr}
\hline Locomotive sill velocity (mph) & 30 & 60 \\
Locomotive sill velocity (in/sec) & 528 & 1056 \\
Duration of impact (sec) & 0.0125 & 0.0135 \\
Velocity at end of impact (in/sec) & 575 & 1130 \\
g load on cask (g) & 138 & 265 \\
Maximum effective stress (psi) & 50,000 & 65,000 \\
Maximum plastic strain (\%) & 12.4 & 20 \\
Maximum plastic strain at inner shell (\%) & 3.7 & 6.0 \\
\hline
\end{tabular}




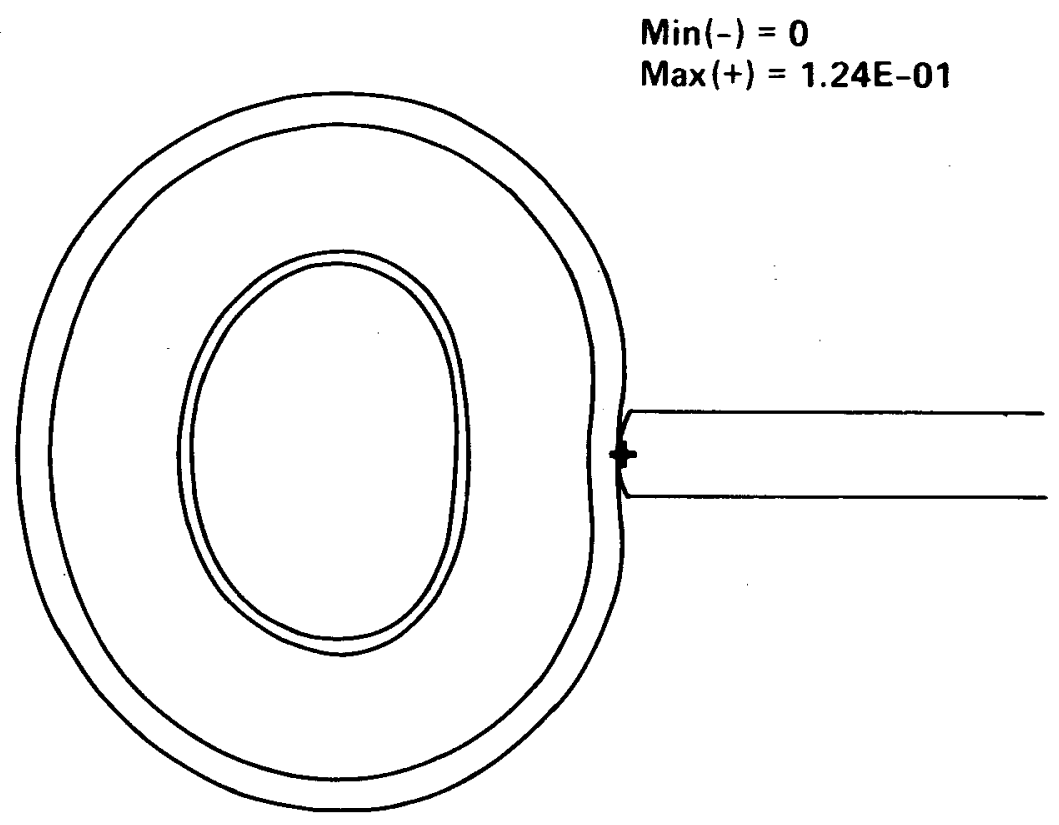

Figure E-39 Thirty mph sidewise head-on sill impact-maximum plastic strain location. 
sidewise head-on impact causes severe local deformations before the cask is accelerated to its final speed. Also, the locomotive sill has some give to it. These combined effects soften the impact. The rail cask endures the least severe stresses, strains, and g loads, yet it sustains the most severe deformations. This is because the rail cask is more ductile than the truck cask, causing a very soft impact.

\section{E.7.4.2 Impact on Rail Cask}

The response of the representative rail cask was estimated for impacts with a train sill by using the truck cask results. The response was estimated by multiplying the truck cask results for the train sill impact times the rail cask results for the unyielding surface impact and dividing by the truck cask results for the unyielding surface impact. The estimated responses of the representative rail cask to impacts by a train sill are summarized in Table E.27. The estimated strains are conservatively high because of the greater size and weight of the rail cask compared to the truck cask. 
Table E.27

Estimated Response of Rail Cask to Impact by Train Sill

\begin{tabular}{lcc}
\hline & \multicolumn{2}{c}{$\begin{array}{c}\text { Strain Response } \\
(\%)\end{array}$} \\
\cline { 2 - 3 } $\begin{array}{c}\text { Velocity } \\
\text { (mph) }\end{array}$ & $0^{0}$ & $45^{\circ}$ \\
\hline 30 & & \\
60 & 2.3 & 1.4 \\
\hline
\end{tabular}

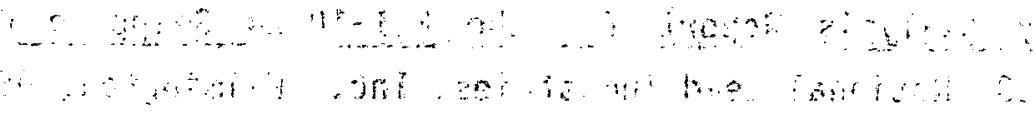




\section{E.8 References}

E.1 J. 0. Hallquist, NIKE 2-D: An Implicit, Finite-Deformation, Finite Element Code for Analyzing the Static and Dynamic Response of TwoDimensional Solids, Lawrence Livermore National Laboratory, Livermore, CA, UCRL-52678, 1979, and Revision 1, NIKE 2-D: An Implicit, FiniteDeformation, Finite Element Code for Analyzing the Static and Dynamic Response of Two-Dimensional Solids, Lawrence Livermore National Laboratory, Livermore, CA, UCID-18822, 1981.

E.2 J. 0. Hallquist, User's Manual for Dyna 2-D--An Explicit TwoDimensional Hydrodynamic Finite Element Code with Interactive Rezoning, Lawrence Livermore National Laboratory, Livermore, CA, UCID-18756, Rev. 2, 1984 .

E.3 American Society of Mechanical Engineers, ASME Boiler and Pressure Vessel Code, Section III, Division 1, The American Society of Mechanical Engineers, United Engineering Center, 345 East 47th Street, New York, NY 10017, July 1983.

E.4 J. B. Conway, R. h. Stentz, and J. T. Berling, Fatigue, Tensile, and Relaxation Behavior of Stainless Steels, Technical Information Center, office of Information Services, United States Atomic Energy Commission, Oak Ridge, TN, 1975.

E.5 Safety Analysis Report for the NLI-10-24 Shipping Cask, Docket No. 70-9023, National Lead Industries, Inc., Wilmington, DE, February 1976.

E.6 J. Counts and J. B. Payne, Evaluation of Analys is Methods for Type B Shipping Container Impact Problems, Los Alamos Scientific Laboratory, Los Alamos, NM, LA-6640-MS, 1979. 
E.7 Consolidated Safety Analys is Report IF-300 Shipping Cask "Appendix I", Docket No. 70-1220, General Electric Company, San Jose, CA, January 1971.

E.8 C. R. Adams, et al, A Comparison of Analytical Techniques for Analyzing a Nuclear-Spent-Fuel Shipping Cask Subjected to an End-on Impact, U.S. Nuclear Regulatory Commission, Washington, DC, NUREG/CR-2018, 1981.

E.9 P. Eggers, Severe Rail and Truck Accidents: Toward a Definition of Bounding Environments for Transportation. Packages, U.S. Nuclear Regulatory Commission, Washington, DC, NUREG/CR-3498, 1984.

E.10 N. E. Shoemaker, et al., Consumer Information Crash Test Program, U.S. Department of Transportation, Washington, DC, DOT HS-802011, September 1976.

E.11 M. Huerta, Analysis, Scale Modeling, and Full-Scale Tests of a Truck Spent-Nuclear-Fuel Shipping System in High Velocity Impacts Against a Rigid Barrier, Sandia National Laboratory, Albuquerque, NM, SAND77-0270, 1978.

E.12 M. Huerta, Analysis, Scale Modeling, and Full-Scale Tests of a Railcar and Spent-Nuclear-Fuel Shipping Cask in a High Velocity Impact Against a Rigid Barrier, Sandia National Laboratory, Albuquerque, NM, SAND780458, Febuary 1980.

E.13 Uniform Building Code, International Conference of Building Officials, Whittier, California, 1979.

E.14 T. A. Nelson, et. a 1., SCANS - Shipping Cask Analysis System, Vol. 1, Impact Analysis Code User's Manual, Lawrence Livermore National Laboratory, Livermore, CA, UCID-20674/Vol. 1, Draft Report to be published, U.S. Nuclear Regulatory Commission, Washington, DC, NUREG/CR-4554, 1986. 
E.15. J. 0. Hallquist, MAZE, An Input Generator for DYNA 2-D and NIKE 2-D, Lawrence Livermore National Laboratory, Livermore, CA, UCID-19029, June 1983.

E.16. M. A. Gerhard, SLIC, Interactive Graphic Mesh Generator, Lawrence Livermore National Laboratory, Livermore, CA, UCRL-52823, September 1979.

E.17 G. F. Sowers, Introductory Soil Mechanics and Foundations: Geotechnical Engineering, Fourth Edition, MacMillan Publishing Company, New York, NY, 1979.

E.18 J. M. Duncan, P. Byrne, K. S. Wong, and P. Mabry, Strength, StressStrain and Bulk Modulus Parameters for Finite Element Analyses of Stresses and Movements in Soil Masses, University of California at Berkeley, Berkeley, CA, UCB/GT/80-01, 1980.

E.19 W. Yoder, Principles of Pavement Design, Second Edition, J. Wiley and Sons, New York, NY, 1975.

E.20 C. W. Young, "Depth Prediction for Earth-Penetrating Projectiles", Journal of the Soil Mechanics and Foundations Division, Proceedings of the American Society of Civil Engineers Vo1. 95, No. SM3, Proceedings Paper 6558, American Society of Civil Engineers, New York, NY, May 1969.

E.21 J. E. Bowles, Foundation Analys is and Design, Second Edition, McGrawHill, New York, NY, 1977

E.22 H. Wagner, Über Stoss und Gleitvorgange an der oberfläche von Flussigkeiten," Zeitschrift Fur Angewandte Mathematik und Mechanik, August 1932. 
E.23 S. L. Chuang, "Experiments on STamming: of Wedge-Shaped Bodites, " Journal of Ship Research, September 1967. 


\section{APPENDIX $\mathbf{F}$}

List of Figures

F-1 Fire modeling of casks ............................... F-3

F-2 Modeled cask dimensions for TACO input $\ldots \ldots \ldots \ldots \ldots \ldots \ldots \ldots \ldots \ldots \ldots \ldots$ F-8

F-3 Lead mid-thickness temperatures for truck cask versus duration of regulatory fire ................................ F-14

F-4 Lead mid-thickness temperatures for rail cask versus duration of regulatory fire $\ldots \ldots \ldots \ldots \ldots \ldots \ldots \ldots \ldots \ldots \ldots \ldots \ldots \ldots \ldots$

F-5 Initial heat flux on truck cask for various fire conditions

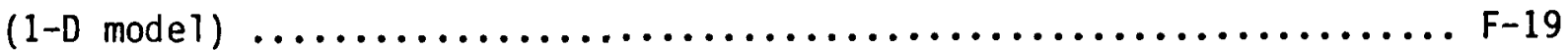

$\mathrm{F}-6$ Heat flux on truck cask versus duration of $1475^{\circ} \mathrm{F}$ and $1825^{\circ} \mathrm{F}$ fires .... F-21

F-7 Integrated heat flux on truck cask versus duration of

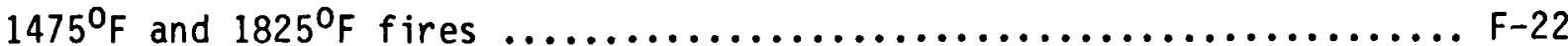

F-8 Lead mid-thickness temperature for truck cask versus duration of $1825^{\circ} \mathrm{F}$ fire ................................... F-23

F-9 Heat flux on rail cask versus duration of $1475^{\circ} \mathrm{F}$ and $1825^{\circ} \mathrm{F}$ fires .... F-25

F-10 Lead mid-thickness temperature for rail cask versus

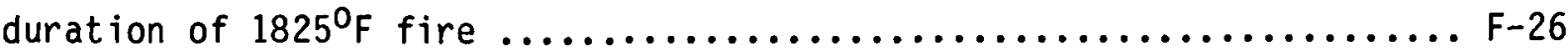

F-11 Initial and average heat flux on truck and rail cask versus flame temperature $\ldots \ldots \ldots \ldots \ldots \ldots \ldots \ldots \ldots \ldots \ldots \ldots \ldots \ldots \ldots \ldots \ldots$. . . . 27

F-12 Cask on ground with tangent flame front $\ldots \ldots \ldots \ldots \ldots \ldots \ldots \ldots \ldots \ldots \ldots$ F-29

F-13 Cask on ground--distant from flame front .....................F-30

F-14 Fraction of heat load from nonengulfing fires $\ldots \ldots \ldots \ldots \ldots \ldots \ldots \ldots \ldots, F-32$

List of Tables

F.1 Material Thermal Properties ..................................F-11

F.2 Internal Heating from Fuel Asse mblies $\ldots \ldots \ldots \ldots \ldots \ldots \ldots \ldots \ldots \ldots \ldots \ldots \ldots \ldots$ F-12 


\section{APPENDIX $F$}

Thermal Analysis

\section{F.1 Introduction}

This appendix provides the thermal models developed and the analyses performed to determine the responses of the representative truck and rail casks to a wide range of fire conditions. The computer code TACO-2D was used to perform the calculations. F.1

In Section F.2, the types of thermal loading conditions that can effect the temperature response of a cask in an accident are discussed. The highest loading condition is caused by large, long-duration fires and is selected for further evaluation. In Section F.3, the thermal loading conditions on a cask caused by real fire conditions are discussed. Referenced fire conditions and modeling are defined for evaluating real fire effects on casks.

In Section F.4, the thermal model and transient temperature response of the two representative casks to regulatory fire conditions are discussed. In Section F.5, the transient temperature response of the two representative casks is estimated for different heat loading conditions and a wide spectrum of real fire conditions defined by fire duration, temperature, and location.

\section{F.2 Thermal Loading Conditions Caused by Accidents}

Thermal loading conditions on a cask caused by an accident can result in cask temperature increases... The thermal loading conditions include fires, torch fires, and cask burial. Typically, as discussed in Section F.5, a fire can heat a cask at an average heat flux of 5,000 . Btu/hhr-ft ${ }^{2}$ from several minutes to several hours. The total heat absorbed in a fire can be 1,000 to $50,000 \mathrm{BTU} / \mathrm{ft}^{2}$ depending on the fire temperature, location, and duration.

Torch fires can heat $a^{r}$ loca 1 izedsarea of alicas at rates 1.5 to 2.5 times higher than a fire, but inn comparison sto fires, do not deposit large quantities of heat into the cask: As demontrated in torch fire tests at Sandia,F.2 no significantuliocalized damage occurs to the cask even at the high heating fluxes because the heat is quickly dissipated to other portions of the cask thus limiting the rise in the local temperature.

$$
\text { F-1 }
$$


Burial of the cask can cause the temperature of the cask and contents to rise because of the decay heat from the fuel. Burial of the cask can cause thermal isolation, where the decay heat from the fuel may have to be transferred through the surrounding material causing the burial. The decay heat flux from the fuel in a cask is typically 50-350 Btu/hr-ft ${ }^{2}$ depending primarily on the number of fuel assemblies, their burnup, and their time out of a reactor. The decay heat flux from the fuel assemblies is 15-50 times lower than that which can be typically absorbed from a fire. The cask, which is relatively large and not easily buried, would have to be buried for several days before any significant damage to the cask could occur that could result in radioactive releases.

Based on severe accident data, the frequency of occurrence of fires is at least 10 times higher than for torch fires or complete burial of a cask. Therefore, since fires can generate higher heat loads and can occur more frequently, it is concluded that fires dominate the potential thermal environment and only fires require further evaluation.

\section{F.3 Reference Fire Conditions and Modeling}

In Fig. F-1(a), a three-dimensional (3-D) model of a cask engulfed in a real fire is given. The heat transfer from the fire to the cask can vary with time and position along the length and around the diameter of the cask. The effects of the fire can be significantly different on the various components located on the cask. To simplify the heating analysis of the cask and its components, currently licensed cask designs were reviewed to relate the temperatures at the middle portion of the cask to the temperatures of the other positions of the cask, particularly the closure seals. The location of valve boxes was also considered because they could be exposed to heat loads and temperatures approaching this middle portion of the cask. From this review, it was concluded that the temperature response and damage to the cask and its components could be conservatively bounded by analyzing the middle portion of the cask and using the four temperature response levels defined in Section 4.0 for the centerline of the lead shielding. Using this approach, the 3-D model in Fig. $F-1(a)$ is reduced to the two-dimensional (2-D) model in Fig. $F-1(b)$ for analysis. 


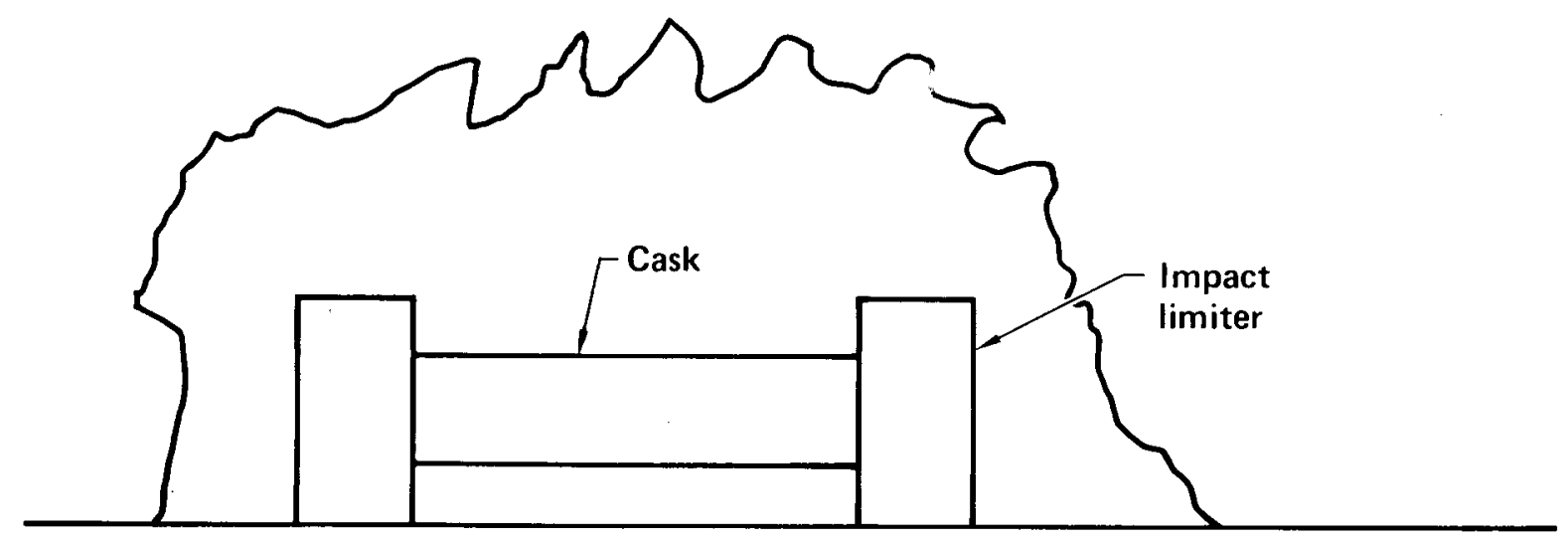

a) Three-dimensional cask fire model

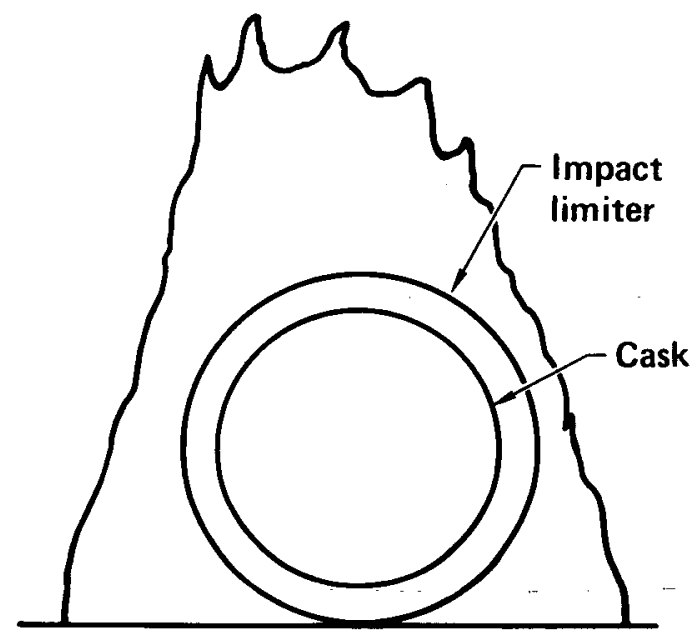

b) Two-dimensional cask fire model

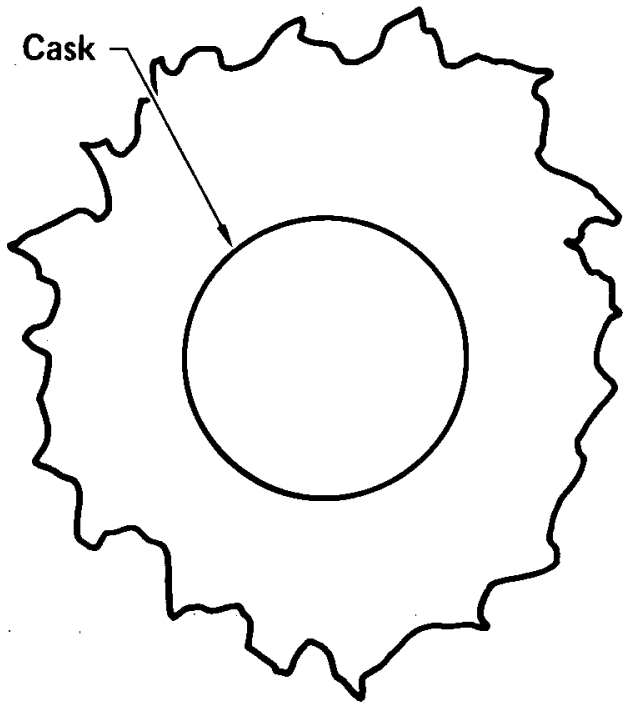

c) One-dimensional cask fire model

Figure F-1 Fire modeling of casks. 
In a real engulfing fire, the spent fuel cask is partially shielded from the heat by either the transport vehicle or the ground. In real fires the amount of heat transferred to the cask differs significantly from that from a hypothetical totally engulfing fire, represented by the one-dimensional (1-D) model in Fig. $\mathrm{F}-1(\mathrm{C})$.

The role of convection from the flame may be significant for cases in which the cask is enclosed within or very near the flame while on either the ground or the vehicle. There does not appear to be sufficient experimental evidence to formulate any general rule to evaluate convection coefficients in this geometry. Also the flame temperature can vary significantly along the diameter of the cask. A common analytical approach is to consider the flame to be isothermal, with a single value for emissivity and a conservatively high temperature to attempt to account for the convective effects, since these are the most highly variable and difficult effects to measure and to model.

In the case of engulfing fires, the radiative heat load from an isothermal fire to the cask can be calculated as follows: F. 3

$$
\dot{Q}_{r}=\sigma A_{s} C_{s-f}\left(T_{f}^{4}-T_{s, 0}^{4}\right)
$$

where

$$
\begin{aligned}
\dot{Q}_{r} & =\text { radiant heat load per unit length and time, Btu/ft-hr } \\
\sigma & =\text { Stefan-Boltzman constant, Btu/hr- } \mathrm{ft}^{2}-{ }^{\circ} \mathrm{K}^{4} \\
\mathrm{C}_{\mathrm{S}-\mathrm{f}} & =\text { configuration factor, unitless } \\
\mathrm{A}_{\mathrm{S}} & =\text { area of cask exposed to flame, } \mathrm{ft}^{2} / \mathrm{ft} \\
\mathrm{T}_{\mathrm{f}} & =\text { flame temperature, absolute, }{ }^{\circ} \mathrm{R}
\end{aligned}
$$


$T_{S, 0}=$ initial cask surface temperature, absolute, ${ }^{\circ} \mathrm{R}$

For a real fire the configuration factor for two gray, diffuse bodies exchanging heat is given by:

$$
A_{s} C_{s-f}=\frac{1}{\frac{1-\varepsilon_{f}}{\varepsilon_{f} A_{f}}+\frac{1}{A_{s} F_{s-f}}+\frac{1-\varepsilon_{s}}{\varepsilon_{s} A_{s}}}, \mathrm{ft}^{2} / f t
$$

where

$$
\begin{aligned}
A_{f} & =\text { area of flame involved, } \mathrm{ft}^{2} / \mathrm{ft} \\
\mathrm{F}_{s-f} & =\text { geometric view factor from cask to fire, unitless } \\
\varepsilon_{f} & =\text { flame emissivity }=0.9, \text { unitless } \\
\varepsilon_{s} & =\text { cask surface emissivity }=0.8, \text { unitless }
\end{aligned}
$$

and all other terms are as previously defined.

If it is assumed as shown in Fig. F-1(b) that no significant fire exists below the horizontal centerline and within the diametral dimension of the cask, the geometric view factor from the cask to the fire below the centerline for one side of the lower portion of the cask is given by:

$$
\left(A_{s} F_{s-f}\right)_{B}^{3}=\frac{\pi r}{4}, f t^{2} / f t
$$

where

$$
r=\text { radius of cask, ft }
$$


for a 2-D infinitely long cylinder. The area-configuration factor calculated using Equation F.2 is:

$$
\left(A_{s} C_{s-f}\right)_{B}=\frac{2 \pi}{\frac{1-\varepsilon_{f}}{\varepsilon_{f}}+\frac{2}{\pi}\left(\frac{1+\varepsilon_{s}}{\varepsilon_{s}}\right)}, \mathrm{ft}^{2} / \mathrm{ft}
$$

Assuming that the cask is completely engulfed by the fire above the centerline, the area-configuration factor above the centerline is given by:

$$
\left(A_{s} C_{s-f}\right)_{A}=\pi r \bar{\varepsilon}, f t^{2} / f t
$$

where

$$
\bar{\varepsilon}=\text { effective emissivity }=\overline{1} / \varepsilon_{f}+-\frac{1}{1 / \varepsilon_{s}-1} \text {. }
$$

Adding the results of Equations $F .4$ and $F .5$ together, the total areaconfiguration factor for a real fire is:

$$
\left(A_{s} C_{s-f}\right)_{T}=\frac{2 \pi}{\frac{1-\varepsilon_{f}}{\varepsilon_{f}}+\frac{2}{\pi}\left(\frac{1+\varepsilon_{s}}{\varepsilon_{s}}\right)}+\pi r \bar{\varepsilon}, \mathrm{ft}^{2} / \mathrm{ft}
$$

A hypothetical regulatory engulfing fire is shown as a $1-0$ fire in Fig. $F-1(c)$. The regulatory fire is defined as having a fire temperature of $1475^{\circ} \mathrm{F}$, a flame emissivity of 0.9 , and a fire duration of 0.5 hour. The areaconfiguration factor for the regulatory fire is: 


$$
\left(A_{s} C_{s-f}\right)_{T}=2 \pi r \bar{\varepsilon} \quad, \quad f t^{2} / f t
$$

Then the ratio of the heat load of real fires to a hypothetical uniform fire is the ratio of Equations F.6 and F.7:

$$
\frac{\dot{Q}_{r}}{\dot{Q}_{h}}=\frac{\left[\frac{1-\varepsilon_{f}}{\varepsilon_{f}}+\frac{2}{\pi}\left(\frac{1+\varepsilon_{s}}{\varepsilon_{s}}\right)\right]}{r \bar{\varepsilon}}+1 / 2=0.78
$$

for the same flame emissivity of 0.9 , cask surface emissivity of 0.8 , fire temperature, and cask surface temperature.

Based on Equation F.8, a higher flame temperature is required for the cask to absorb the same amount of heat for a real fire compared to a hypothetical fire. As derived in. Section F.5, the hypothetical regulatory fire with a fire temperature of $1475^{\circ} \mathrm{F}$ generates the same heat load on a cask as a $1700^{\circ} \mathrm{F}$ real fire. The reference fire conditions are defined to be the $1700^{\circ} \mathrm{F}$ real fire that generates the same heat load as the regulatory fire. The 1-D model (Fig. $F-1(C)$ ) can be used to approximate the 2-D model (Fig. $F-1(b)$ ) provided that the heat loading conditions are appropriately accounted for.

\section{F.4 Cask Temperature Response to Regulatory and Reference Fire Conditions}

The transient thermat response of a representative truck and rail cask to an engulfing reference fire was analyzed using TACO. $\vec{F} .1$ A I-D model of the casks engulfed by the regulatory fire simplifies the calculation and predicts reasonably well the thermal response of the major volume of the casks. This mode 1 is used to estimate the cask response to the reference $1700^{\circ} \mathrm{F}$ real fire engulfing a cask. Figure F-2 shows the geometry of the modeled casks.

The initial temperature distribution within each cask from heat generated by the spent fuel was established before subjecting the cask to the modeled 


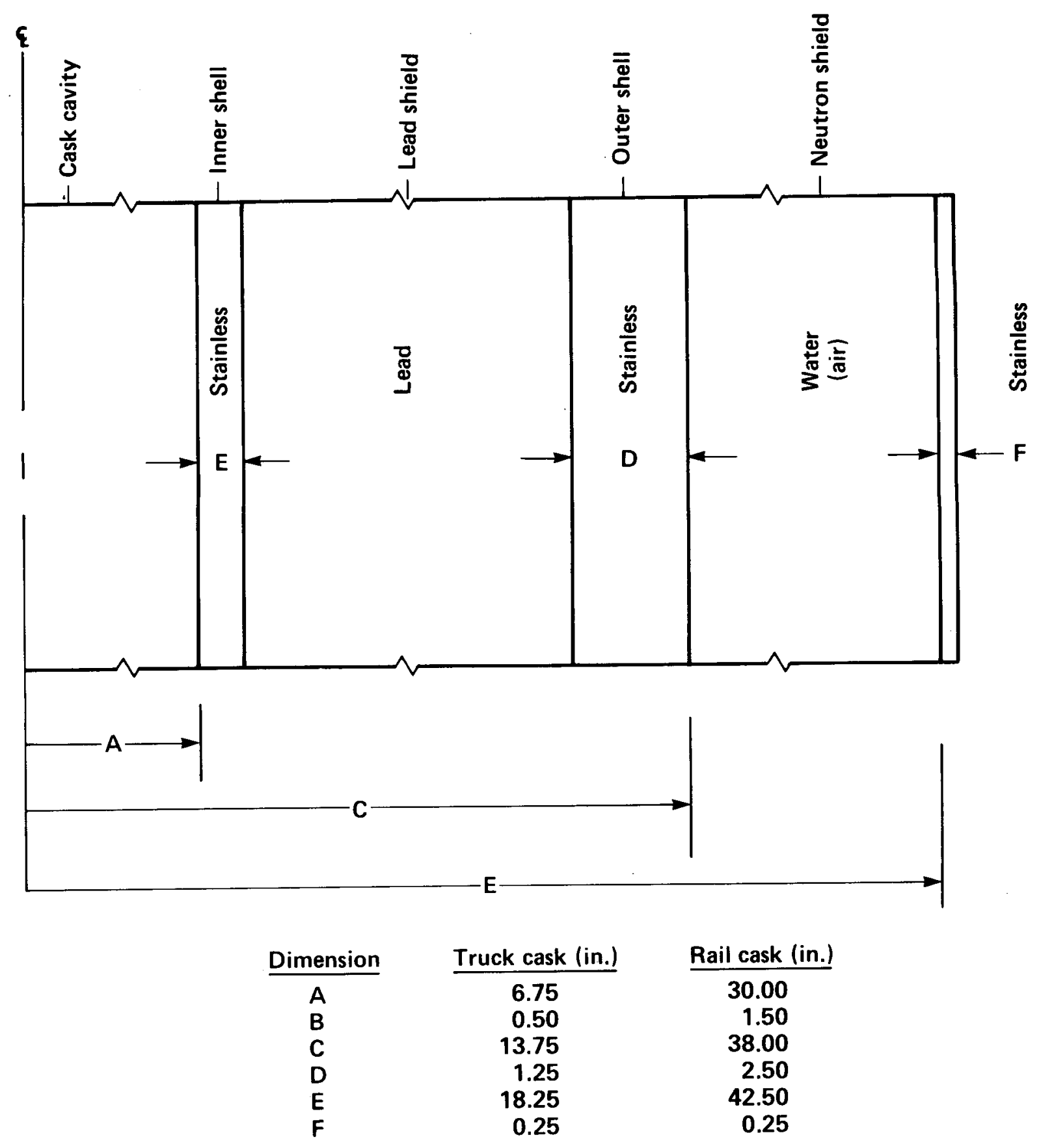

Figure F-2 Modeled cask dimensions for TACO input.

$$
\text { F-8 }
$$


fire environment. The steady-state evaluation was performed using TACO, with the assumption that the neutron shield tank is filled with water. The heat transfer through the water is by conduction and natural convection. A convenient way to model the natural convection is through the use of an effective conductivity for the water. Holman gives a relationship for effective conductivity of a fluid in a horizontal cylindrical annulus as: $F .4$

$$
\frac{k e}{k}=C\left(G r_{\delta} \operatorname{Pr}\right)^{r} \text {, unitiess }
$$

where

$$
\begin{aligned}
k_{e} & =\text { effective thermal conductivity, Btu/hr-ft- }{ }^{\circ} \mathrm{F}, \\
k & =\text { thermal conductivity, Btu/hr-ft- }{ }^{\circ} \mathrm{F}, \\
G r_{\delta} & =\text { Grashof Number }=\frac{g \rho^{2} B \delta^{3} \Delta T}{\mu^{2}}, \text { unitless } \\
\operatorname{Pr} & =\text { Prandtl Number, unitless } \\
g & =\text { gravitational constant, ft/sec }{ }^{2} \\
\beta & =\text { volume coefficient of expansion, } 1 /{ }^{\circ} \mathrm{F} \\
\delta & =\text { annulus width, ft } \\
\rho & =\text { density, lb/ft }{ }^{3} \\
\mu & =\text { dynamic viscosity, lb/sec-ft } \\
\Delta T & =\text { temperature difference across annulus., }{ }^{\circ} \mathrm{F}
\end{aligned}
$$




$$
\begin{aligned}
r= & 0.29 \text { for } 6 \times 10^{3} \leqq \mathrm{GrPr}<10^{6} \\
& 0.20 \\
C= & 0.11 \text { for } 6 \times 10^{6} \leqq \mathrm{GrPr}<10^{8} \leqq \mathrm{GrPr}<10^{6} \\
& 0.40 \quad 10^{6} \leqq \mathrm{GrPr}<10^{8} .
\end{aligned}
$$

This expression was evaluated over the expected temperature range, and an average value of effective conductivity of water as a function of bulk temperature was used.

Table F.1 tabulates the material thermal properties used in the analysis. Table F.2 lists the internal heat assumed for the fuel assemblies within the two casks. A uniform value of $1.0 \mathrm{Btu} / \mathrm{hr}-\mathrm{ft}^{2}{ }^{\circ}{ }^{\circ} \mathrm{F}$ was used to represent natural convective heat removal from the cask surface.

The results of the steady-state analys is for the casks show a surface temperature of $147^{\circ} \mathrm{F}$ for the truck and $242^{\circ} \mathrm{F}$ for the rail cask.

For the regulatory fire, only radiation heat transfer occurs. The heat flux from a hypothetical engulfing fire on the surface of the cask due to radiation heat transfer is given by:

$$
q=\sigma \tilde{\varepsilon}\left(T_{f}^{4}-T_{s}^{4}\right), B t u / h r-f t^{2}
$$

where

$$
T_{s}=\text { cask (neutron shield) surface temperature, absolute, }{ }^{\circ} R
$$

and all other terms are as previously defined.

It is next assumed that before being engulfed by fire, the water leaks out of the neutron shield tank. Heat transfer in the annulus is now through the combined modes of radiation across the gap and convection and conduction 
Table F.1

Material Thermal Properties

\section{Stainless Steel. Density \\ Temperature

\begin{tabular}{c}
$\left({ }^{\circ} \mathrm{F}\right)$ \\
\hline 50 \\
250 \\
500 \\
750 \\
1000 \\
1250 \\
1500 \\
2372
\end{tabular}

\section{Lead}

Dens ity

Melt Point

Latent Heat

Temperature

$\left({ }^{\circ} \mathrm{F}\right)$

50

250

619

1500

1832
$494.2 \mathrm{lb} / \mathrm{ft} \mathrm{t}^{3}$

Therma 1 Conductivity

$\frac{\left(B t u / h r-f t-{ }^{\circ} \mathrm{F}\right)}{7.92}$

8.64

9.72

10.86

12.06

13.5

14.46

16.92
Specific Heat

(Btu:i1b)

0.107

0.11 .

0.120

0.133

0.138

0.144

0.150

0.170

$708.5 \mathrm{lb} / \mathrm{ft}^{3}$

$621.5^{\circ} \mathrm{F}$

$10.25 \mathrm{Btu} / 1 \mathrm{~b}$

Therma 1 Conductivity

(Btu/hr-ft-o F)

Spec if ic Heat.

19.97

(Btu/ 1b)

19.2

0.031

0.032

10.4

0.0332

8.64

0.034

8.64

0.0328

Water

Density

Specific Heat

$62.431 \mathrm{~b} / \mathrm{ft}^{3}$

$1.0 \mathrm{Btu} / 1 \mathrm{~b}^{\circ} \mathrm{F}$

Temperature

\begin{tabular}{c}
$\left({ }^{\circ} \mathrm{F}\right)$ \\
\hline 104 \\
140 \\
176 \\
212 \\
284
\end{tabular}

Eff. Thermal Conductivit.y. (Btu/hr-ft- ${ }^{\circ} \mathrm{F}$ )
2. 76
3.0 .1
3.25
3.46
4.34 
Table F.2

Internal Heating from Fuel Assemblies

Heat Load

(KBtu/hr)

Truck Cask

6.82

Rai 1 Cask

71.4 
through the air. As in the case for water, the same relationship holds, but a single value of effective thermal. conductivity of the air as a function of bulk temperature can lead to serious errors. The equation for total heat transfer in the annulus is:

$$
q_{a n}=\frac{\sigma\left(T_{s}^{4}-T_{i}^{4}\right)}{\frac{1}{\varepsilon_{i}}+\frac{d_{i}}{d_{0}}\left(\frac{1}{\varepsilon_{0}}-1\right)}+\frac{2 K_{e}\left(T_{s}-T_{j}\right)}{d_{i} \ln \left(d_{0} / d_{i}\right)}, B t u / h r-f t^{2}
$$

where

$$
\begin{aligned}
& d_{i}=\text { neutron shield inner diameter, } f t \\
& d_{0}=\text { neutron shield outer diameter, } f t \\
& k_{e}=\text { effective air thermal conductivity, Btu } / \mathrm{hr}-\mathrm{ft}-{ }^{\circ} \mathrm{F} \\
& \mathrm{T}_{i}=\text { neutron shield inner diameter temperature, absolute, }{ }^{\circ} \mathrm{R}
\end{aligned}
$$

and all other terms are as previously defined.

Solving this equation over the entire expected temperature range for both surfaces of the annulus and then using an interval halving technique results in a constant value for the effective air thermal conductivity, with a maximum root-mean-square error in the total heat transferred of less than $2.5 \%$, for equal surface emissivities between 0.3 and 0.5 .

The temperature response of the representative truck cask was calculated for the regulatory fire with a flame temperature of $1475^{\circ} \mathrm{F}$, a flame emissivity of 0.9 , and a cask surface emissivity of 0.8 . The temperature at the middle of the lead shield thickness is plotted in Fig. F-3. The cask temperature reaches $500^{\circ} \mathrm{F}\left(\mathrm{T}_{1}\right)$ in 1.08 hours and $600^{\circ} \mathrm{F}\left(\mathrm{T}_{2}\right)$ in 1.35 hours. As the lead mid-thickness temperature increases beyond the $600^{\circ} \mathrm{F}\left(\mathrm{T}_{2}\right)$ level, the lead at 


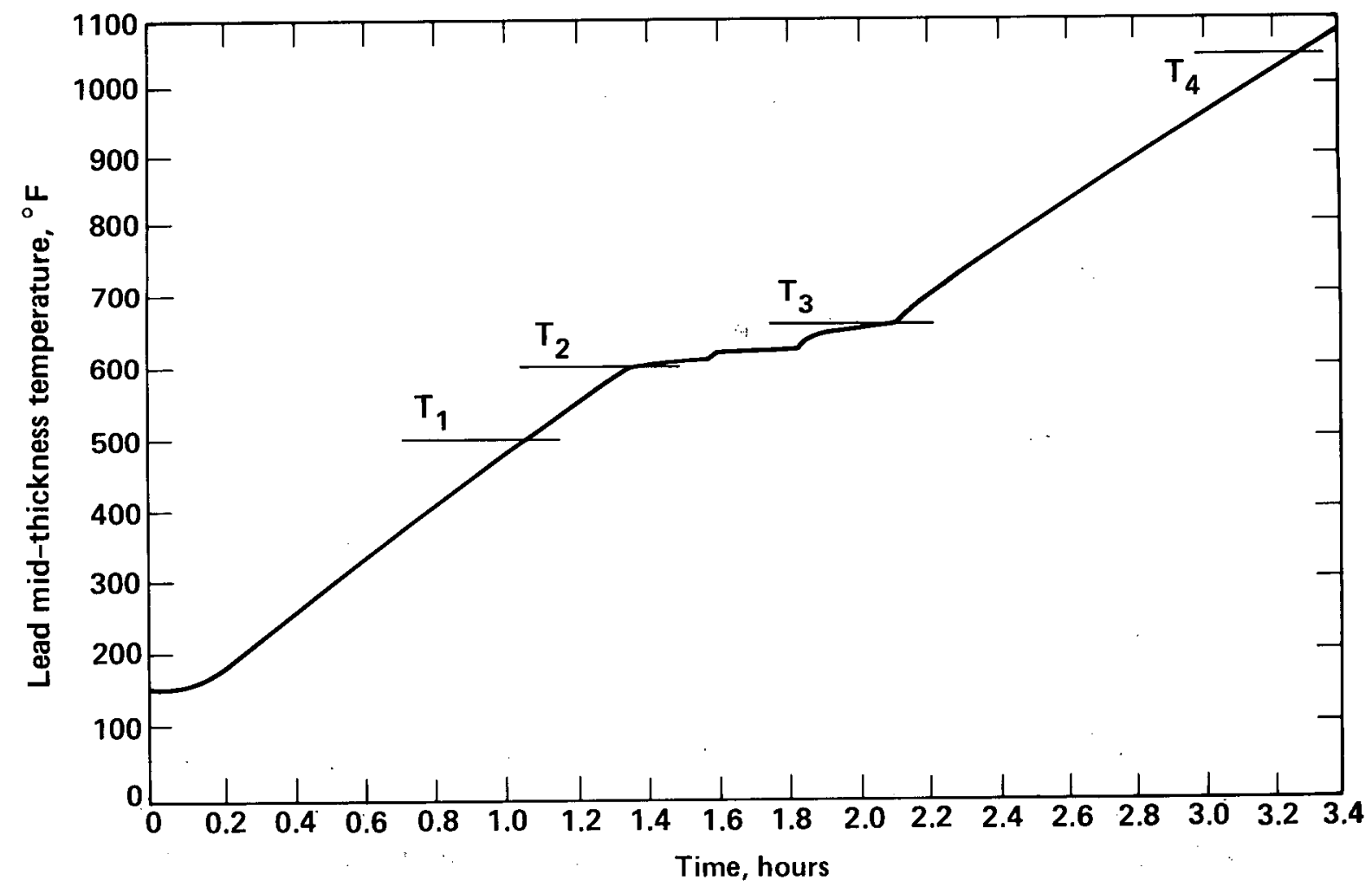

Figure F-3 Lead mid-thickness temperatures for truck cask versus duration of regulatory fire. 
the outer shell starts to melt. The lead melts at the inner shell in 2.1 hours as the mid-thickness temperature reaches $650^{\circ} \mathrm{F}\left(T_{3}\right)$. The $1050^{\circ}$ temperature $\left(\mathrm{T}_{4}\right)$ level is reached in 3.3 hours.

The temperature response of the representative rail cask was also calculated for the hypothetical engulfing fire. The temperature at the middle of the lead shield thickness is plotted in Fig. F-4. The cask temperature reaches $500^{\circ} \mathrm{F}\left(\mathrm{T}_{1}\right)$ in 1.35 hours, and $600^{\circ} \mathrm{F}\left(\mathrm{T}_{2}\right)$ in 1.8 hours. As the read mid-thickness temperature of the lead increases beyond the $600^{\circ} \mathrm{F}\left(\mathrm{T}_{2}\right)$ level, the lead at the outer shell starts to melt. The lead melts at the inner shell in 2.6 hours as the mid-thickness temperature reaches $650^{\circ} \mathrm{F}\left(T_{3}\right)$. The $1050^{\circ} \mathrm{F}$ temperature $\left(T_{4}\right)$ level is reached in 5.1 hours.

These temperature response and heat flux results for the regulatory fire were used to evaluate real fires.

\section{F.5 Cask Response to a Spectrum of Real Fire Conditions}

In order to calculate the thermal response of a cask to a real engulfing fire, certain fire parameters are required. The principal parameters required are fire temperature, flame emissivity, convection velocities, and fire duration. These fire parameters depend upon variables that include type of fuel, amount of fuel, the fuel-air mixture, fire geometry, local temperatures, humidity, and wind conditions. Based on the information provided, the fire temperatures range from 1400 to $2400^{\circ} \mathrm{F}$, flame emissivities range from 0.4 to 1.0 , and convection velocities range from nearly 0 to 20 feet/second. F.5-F.10

The initial heat flux from a hypothetical engulfing fire on the surface of the cask is given by:

$$
\dot{q}=\sigma \tilde{\varepsilon}\left(T_{f}^{4}-T_{s, 0}^{4}\right)+h\left(T_{f}-T_{s, 0}\right), B t u / h r-f t^{2}
$$

where 


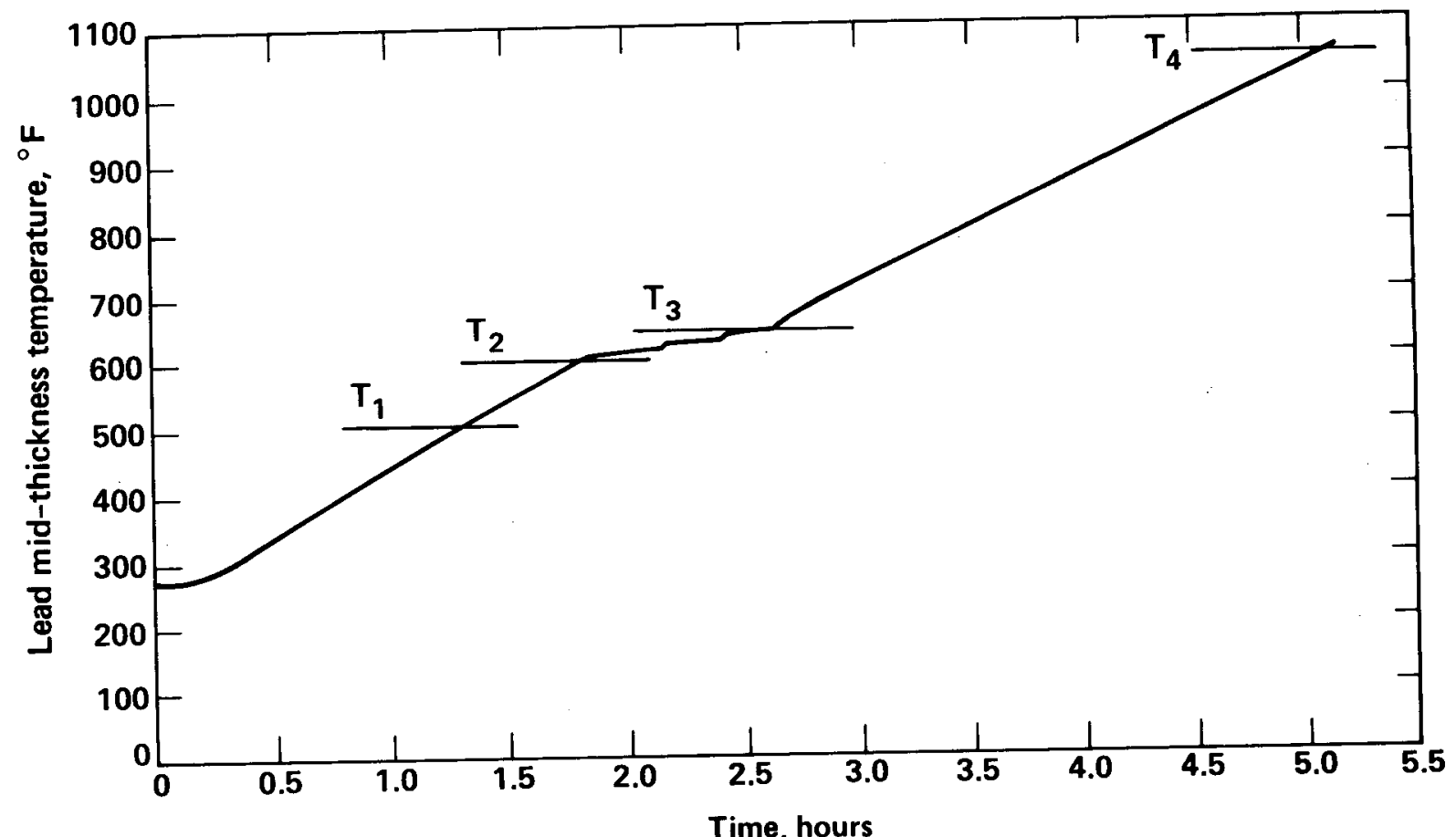

Figure F-4 Lead mid-thickness temperatures for rail cask versus duration of regulatory fire. 


$$
h=\text { convective heat transfer coefficient, Btu/hr-ft }{ }^{2}-{ }^{\circ} \mathrm{F}
$$

and all other terms are as previously defined.

Experimentally determined values for the convection heat transfer coefficient in an engulfing fire have been determined. F.7 The values given for an 8.53 inch diameter cylinder range from 5.2 to $15.8 \mathrm{Btu} / \mathrm{hr}-\mathrm{ft}^{2}-^{\circ} \mathrm{F}$ as a perimeter mean. These values can be scaled within the scaled Reynolds Number by the following relationship:

$$
h=h_{\text {ref }}\left(\frac{{ }^{d} \text { ref }}{d}\right)^{0.195}, \text { BTU } / h r-f t^{2}-{ }^{0} F
$$

where

$$
\begin{aligned}
h_{\text {ref }} & =\text { reference convection heat transfer coefficient, Btu/hr- } \mathrm{ft}^{2}-\mathrm{O}_{\mathrm{F}} \\
d_{\text {ref }} & =\text { reference diameter }=8.53 \text { inches, } \\
d & =\text { diameter }, \text { inches }
\end{aligned}
$$

as long as the scaled Reynolds Number is within the range of applicability. The scaled Reynolds Number is given by:

$$
R_{e}=\operatorname{Re}_{\text {ref }}\left(\frac{d}{d_{\text {ref }}}\right)^{0.805} \text {, unitiess }
$$

where

$$
R_{e}=\text { scaled Reynolds Number, unitless }
$$




$$
\operatorname{Re}_{\text {ref }}=\text { reference Reynolds Number }=73,725 \text {. }
$$

The scaled values of the convection heat transfer coefficient are found to be:

$$
3.9 \text { to } 11.9 \mathrm{Btu} / \mathrm{hr}-\mathrm{ft}^{2}-{ }^{\circ} \mathrm{F} \text { for the truck cask, }
$$

and

3.3 to $10.1 \mathrm{Btu} / \mathrm{hr}-\mathrm{ft}^{2}-^{\circ} \mathrm{F}$ for the rail cask.

Figure F-5 gives the initial heat flux on the surface of the truck cask as a function of flame temperature, flame emissivity, cask emissivity, and convection heat transfer coefficient. This figure provides a wide spectrum of fire conditions which can be related to the regulatory fire conditions in terms of initial heat fluxes. For example, from Fig. F-5, it is determined that an engulfing fire with a $\mathrm{flame}$ temperature of $1300^{\circ} \mathrm{F}$, a flame emissivity of 0.9 , a cask emissivity of 0.8 , and a convection heat transfer coefficient of $5 \mathrm{Btu} / \mathrm{hr}-\mathrm{ft}^{2}-{ }^{\circ} \mathrm{F}$ generates the same initial heat flux to a cask surface as a regulatory fire. For these specific conditions, the initial response of the cask would be essentially the same as its initial response to a regulatory fire. The initial heat fluxes for a rail cask are similar.

A sensitivity study was performed to compare the response of the representative cask for different fire conditions and initial heat fluxes to the responses calculated for the regulatory fire. The initial heat flux to the cask when engulfed by a regulatory fire is:

$$
\begin{aligned}
& \mathrm{q}=17,646 \mathrm{Btu} / \mathrm{hr}^{-\mathrm{ft}^{2}} \text { for the truck cask and } \\
& \mathrm{q}=17,510 \mathrm{Btu} / \mathrm{hr}^{-\mathrm{ft}^{2}} \text { for the rail cask. }
\end{aligned}
$$




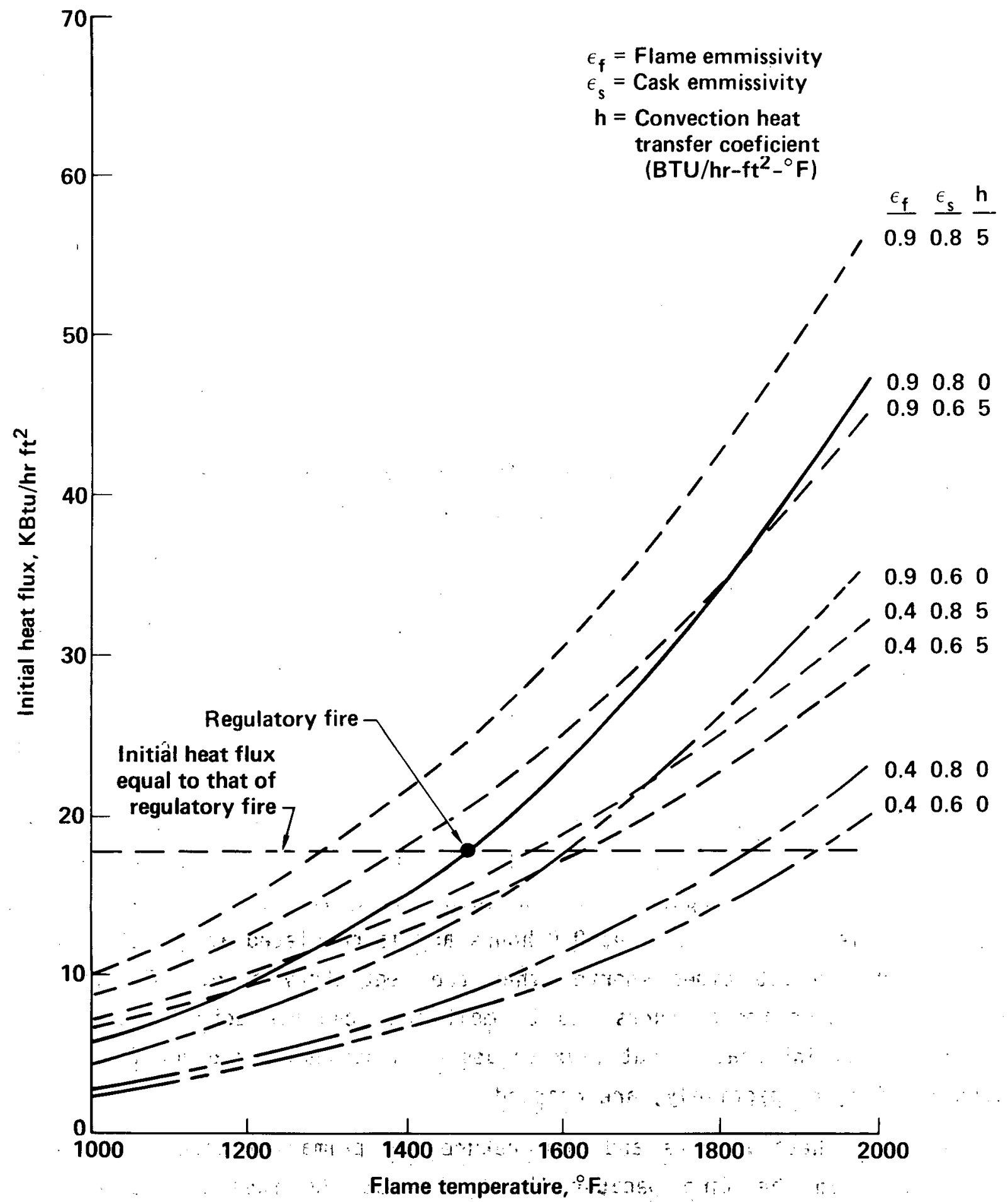

Figure $F-5$ Initial heat flux on truck cask for various fire conditions (1-D model). 
The second fire for comparison was chosen arbitrarily, but within the limits of real fires. The flame temperature was chosen to be $1825^{\circ} \mathrm{F}, f 1$ ame emissivity of 0.9 , and a surface emissivity of 0.8 . The initial heat flux to the cask is:

$$
\begin{aligned}
& q=35,260 \mathrm{Btu} / \mathrm{hr}-\mathrm{ft}^{2} \text { for the truck cask and } \\
& q=34,650 \mathrm{Btu} / \mathrm{hr}-\mathrm{ft}^{2} \text { for the rail cask. }
\end{aligned}
$$

Thus the initial heat flux is about double that caused by the regulatory fire for each of the casks.

The variations of the heat flux for the regulatory and $1825^{\circ} \mathrm{F}$ fires are plotted in Fig. F-6 as a function of time for the truck cask. The heat flux drops rapidly and then decreases slowly because the water jacket acts as a thermal barrier. The heat fluxes after about 1 hour are reduced to 4,500 $\mathrm{Btu} / \mathrm{hr}-\mathrm{ft}^{2}$ for the regulatory fire and $6,750 \mathrm{BTU} / \mathrm{hr}-\mathrm{ft}^{2}$ for the $1825^{\circ} \mathrm{F}$ fire. The integrated heat flux absorbed into the cask is plotted in Fig. F-7 for the regulatory and $1825^{\circ} \mathrm{F}$ fires. The integrated flux rises rapidly at first until the thermal barrier heats up and then limits the heat flux to the cask. The centerline temperatures for the lead shield are plotted in Figs. F-3 and F-8 for the regulatory and $1825^{\circ} \mathrm{F}$ fires, respectively. For the regulatory fire, lead melt starts after 1.35 hours and takes 0.75 hours to complete all the melting. As would be expected for the $1825^{\circ} \mathrm{F}$ fire with a heat flux 1.5 times higher than lead, melt starts at 0.9 hours and is completed after 0.5 hours or times which are 1.5 times shorter than the regulatory fire. The times to reach the melting temperatures and to melt the lead are actually determined when the total integrated heat flux values of approximately $6,000 \mathrm{Btu} / \mathrm{ft}^{2}$ and $9,000 \mathrm{Btu} / \mathrm{ft}^{2}$, respectively, are reached.

The cask heat-up rate and temperature are primarily determined by the heat flux from the fire because the heat from the fuel bundle is about $41 \mathrm{Btu} / \mathrm{hr}-\mathrm{ft}^{2}$. Therefore, it is concluded that the time it takes a specific fire to heat the cask to a specific temperature is approximately proportional to the average heat flux or heat load to the cask. 


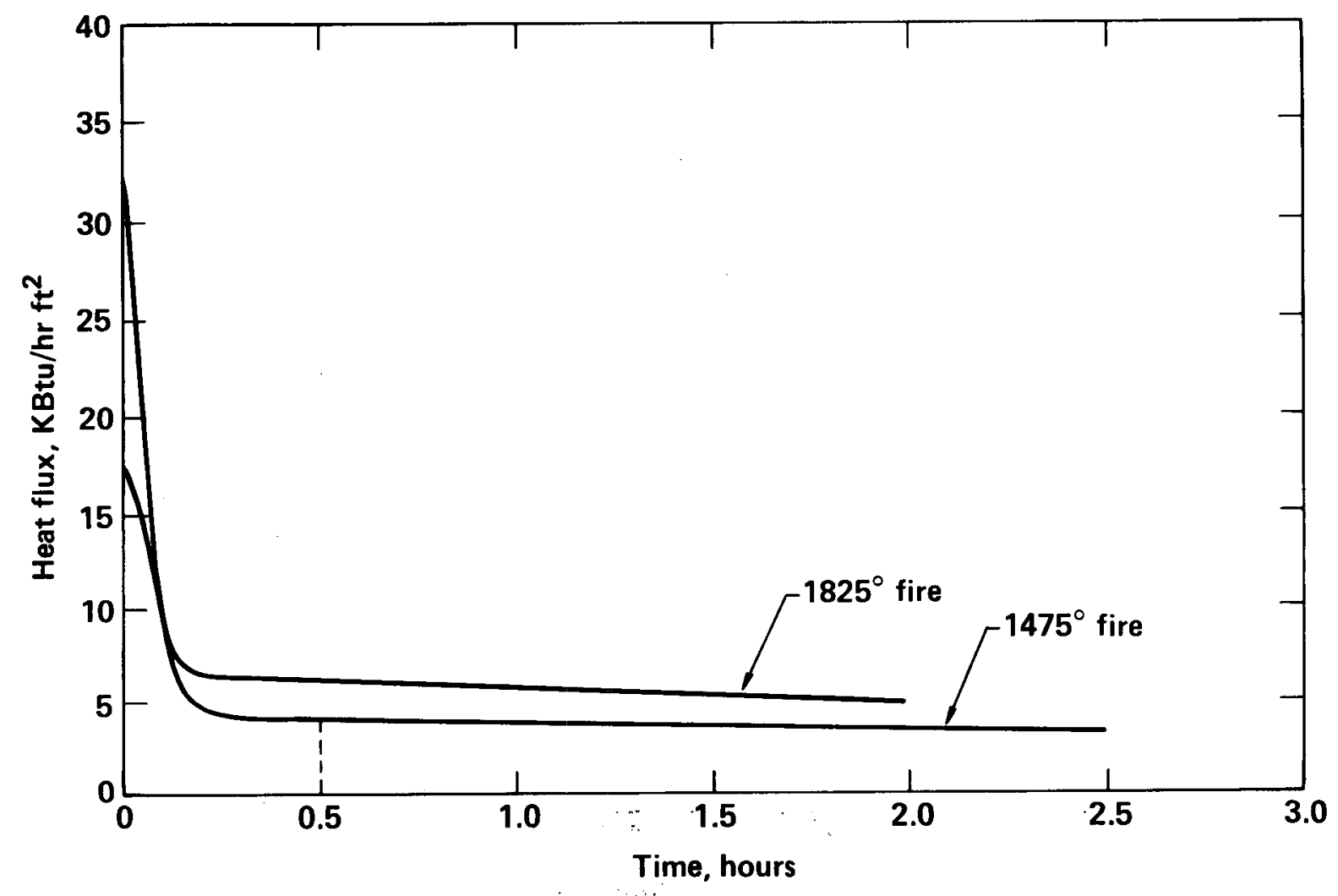

Figure F-6 Heat flux on truck cask versus duration of $1475^{\circ} \mathrm{F}$ and $1825^{\circ} \mathrm{F}$ fires. 


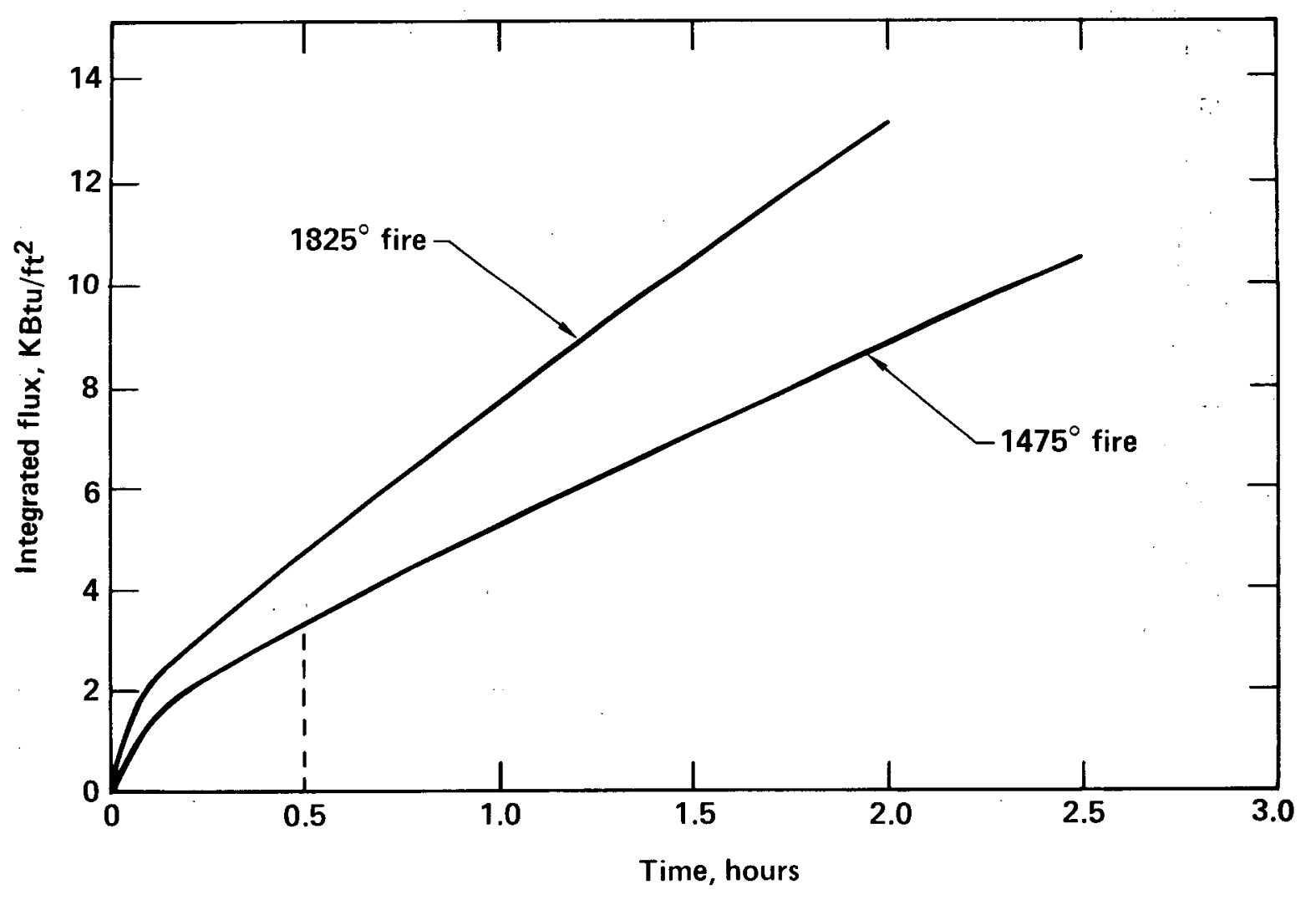

Figure F-7 Integrated heat flux on truck cask versus duration of $1475^{\circ} \mathrm{F}$ and $1825^{\circ} \mathrm{F}$ fires. 


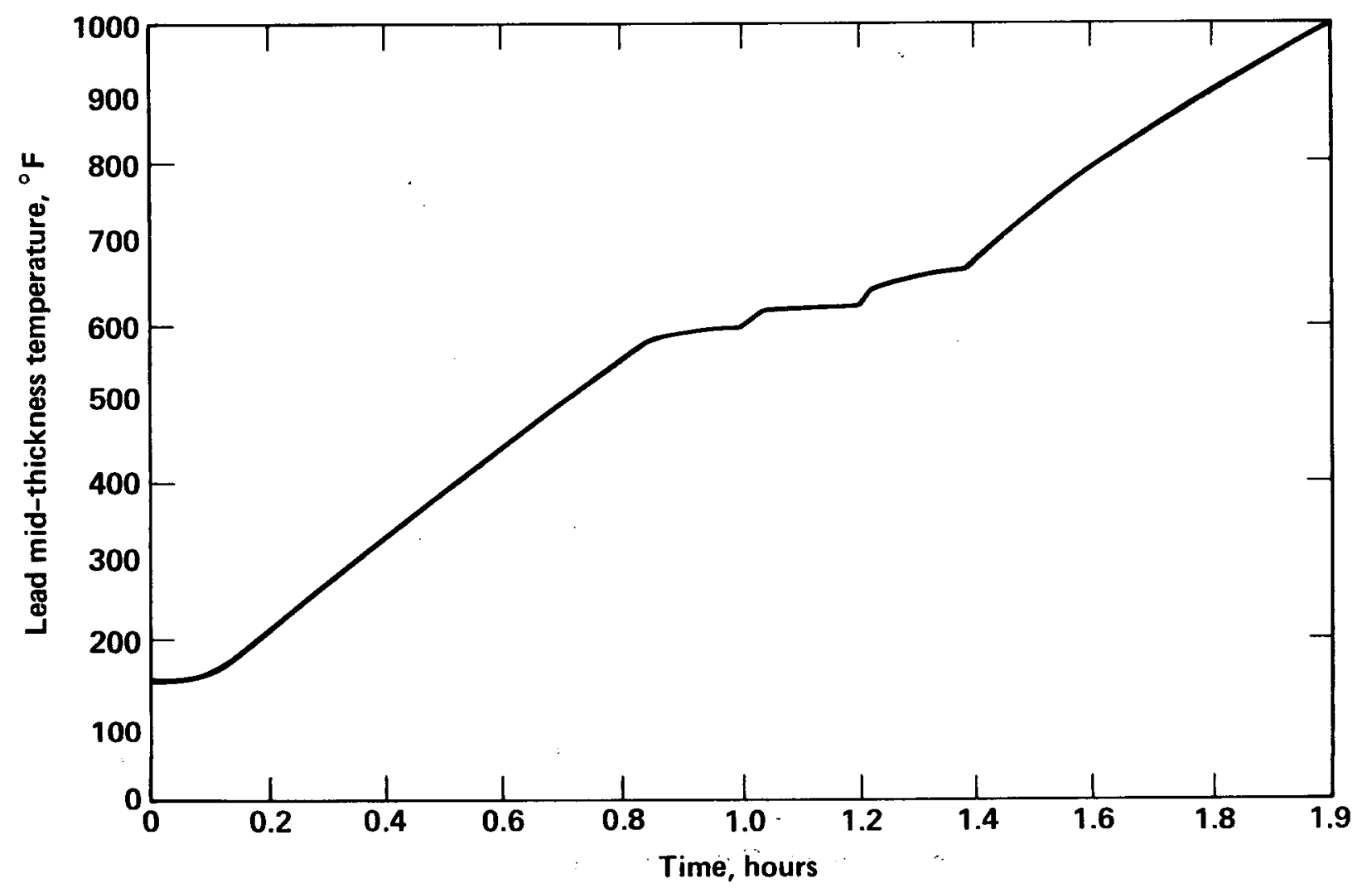

¿ $\because \cdots, \cdots: \cdots: \cdots$

Figure F-8 Lead mid-thickness temperature for truck cask versus duration of $1825^{\circ} \mathrm{F}$ fire.

\section{F-23}


The transient 'thermal analysis for the rail cask was performed in a manner similar to that used for the truck cask. The variations of the heat fluxes for the regulatory and $1825^{\circ} \mathrm{F}$ fires are plotted in Fig. F-9 as functions of time. As with the truck cask case, the heat flux drops rapidly and levels off because the water jacket acts as a thermal barrier. The heat fluxes after about 1 hour are reduced to $4,500 \mathrm{Btu} / \mathrm{hr}-\mathrm{ft}^{2}$ for the regulatory fire and $7,000 \mathrm{BTU} / \mathrm{hr}-\mathrm{ft}^{2}$ for the $1825^{\circ} \mathrm{F}$ fire. These results are similar to those calculated for the truck and indicate that these heat flux values apply to a wide range of cask sizes. The cask will heat up at a rate determined by the heat flux from the fire. The time to reach a particular temperature for the cask is determined by the heat flux. The centerline temperatures for the lead shielding are plotted in Figs. F-4 and F-10 for the regulatory and $1825^{\circ} \mathrm{F}$ fires, respectively. For the regulatory fire, the lead melting begins about 1.8 hours after the fire initiation and is complete at about 2.6 hours. For the $1825^{\circ} \mathrm{F}$ fire, the lead melt begins at 1.2 hours and is complete within 1.8 hours. These melting times are nearly proportional to the fire heat fluxes or heat loads.

In Fig. F-11, the heat flux on the surfaces of the truck and rail cask is plotted as a function of flame temperature, flame emissivity of 0.9 , and cask emissivity of 0.8. The initial heat flux is given. Also, the average heat flux values are given at 1 hour durations for the $1475^{\circ} \mathrm{F}$ and $1825^{\circ} \mathrm{F}$ fires.

As derived in Section F.3, the heat load ratio of a real fire to a hypothetical fire is 0.78 for the same flame temperature. To absorb the same heat load per unit time from a real engulfing fire compared to a hypothetical engulfing fire, the average heat flux on the cask has to be increased. The required heat flux is 1.28 times higher for a real fire. From Fig. F-11 it is determined that a flame temperature of $1700^{\circ} \mathrm{F}$ is required to provide an average flux of $6,400 \mathrm{Btu} / \mathrm{hr}-\mathrm{ft}^{2}$ which is 1.28 times higher than the heat flux derived from regulatory conditions. Therefore, it is concluded that a $1700^{\circ} \mathrm{F}$ real fire provides a heat load to the cask and results in temperature responses similar to those for a $1475^{\circ} \mathrm{F}$ regulatory fire. 


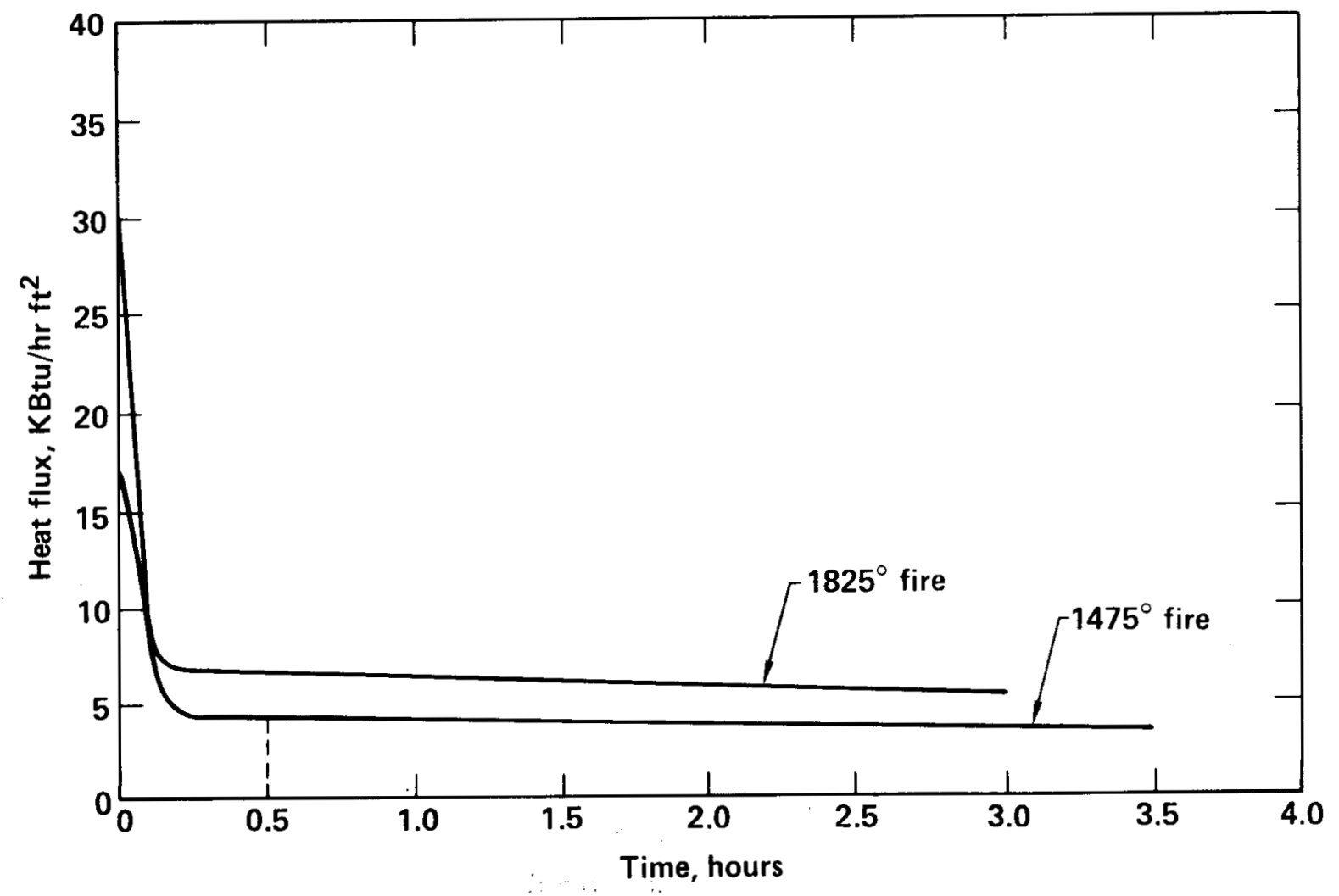

Figure F-9 Heat flux on rail cask versus duration of $1475^{\circ} \mathrm{F}$ and $1825^{\circ} \mathrm{F}$ fires.

$$
F-25
$$




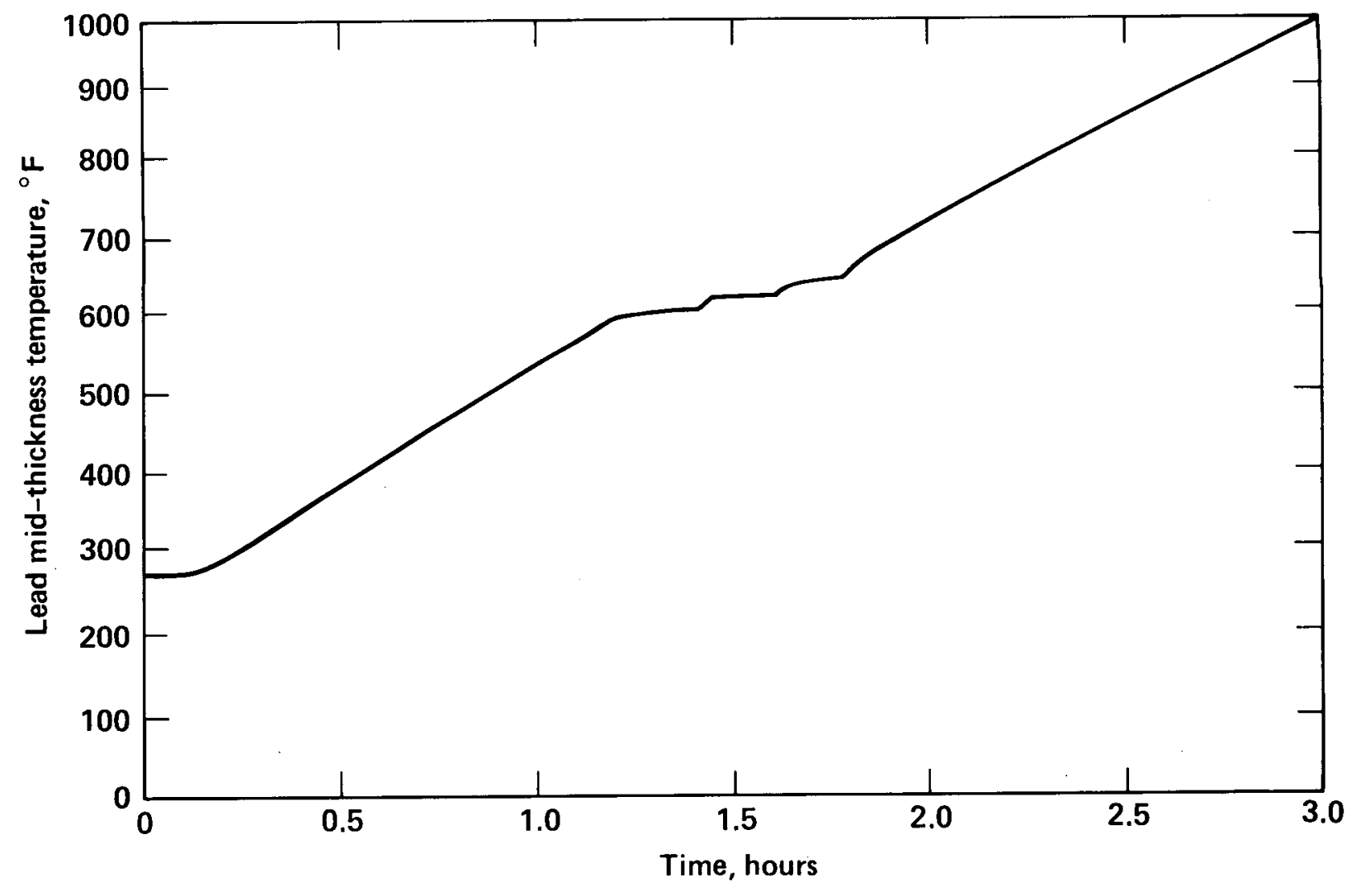

Figure F-10 Lead mid-thickness temperature for rail cask versus duration of $1825^{\circ} \mathrm{F}$ fire. 


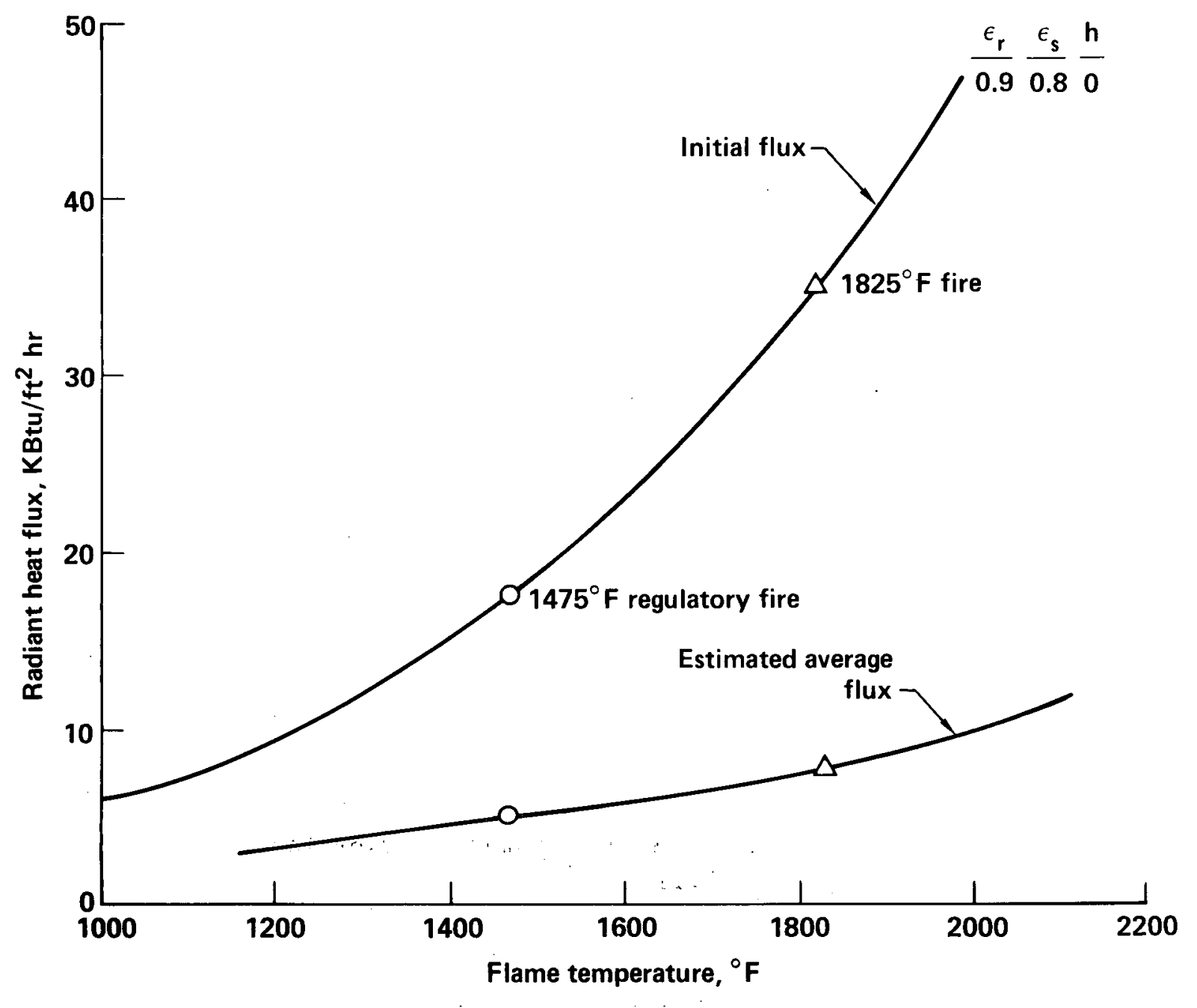

Figure F-11 Initial and average heat flux on truck and rail cask versus flame temperature. 
The heat load to the cask also varies with the location of the fire with respect to the cask. For the case in which the flame front is just tangent to the cask, as shown in Fig. F-12, the geometric view factor to the part of the cask below the horizontal centerline is: F.11

$$
A_{s} F_{s-f}=\frac{\pi r}{4}, f t^{2} / f t
$$

The geometrical view factor to the upper portion of the cask is given by the relationship:

$$
A_{s} F_{s-f}=\frac{r \delta}{2}, f t^{2} / f t
$$

where

$$
\begin{aligned}
& \delta=\pi-2 \tan ^{-1}\left(\frac{r}{h-r}\right), \text { radians } \\
& h=f l \text { ame height, } \mathrm{ft}
\end{aligned}
$$

Finally, for the case in which the cask is removed a distance from the flame front as shown in Fig. F-13, the geometric view factor from the entire cask to the flame is given by: F.11

$$
A_{s} F_{s-f}=r\left[\tan ^{-1}\left(\frac{h-r}{x}\right)+\tan ^{-1}\left(\frac{r}{x}\right)\right], f t^{2} / f t
$$

where

$$
x=\text { separation distance, } f t
$$




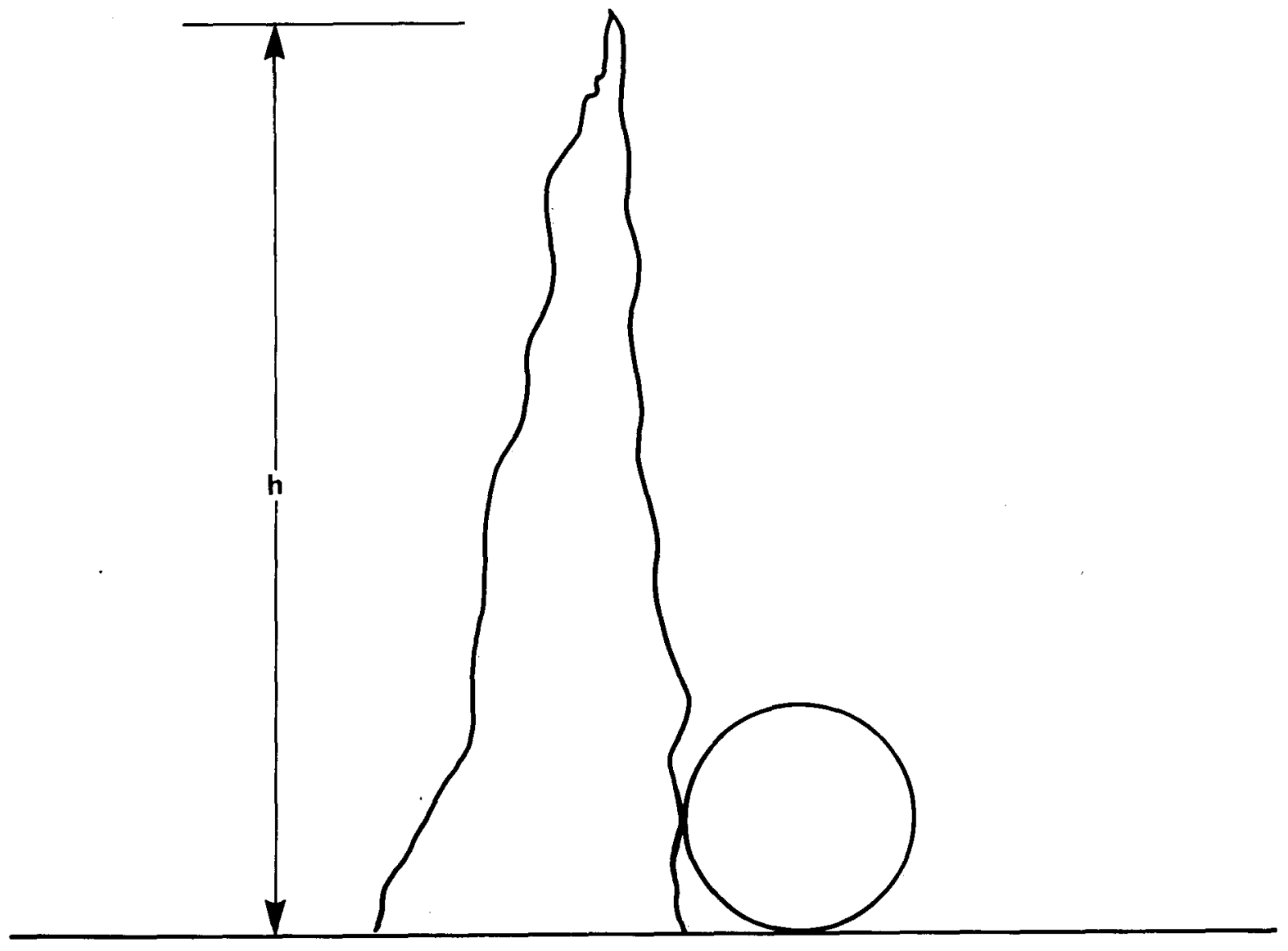

Figure F-12 Cask on ground with tangent. flame front.

F-29 


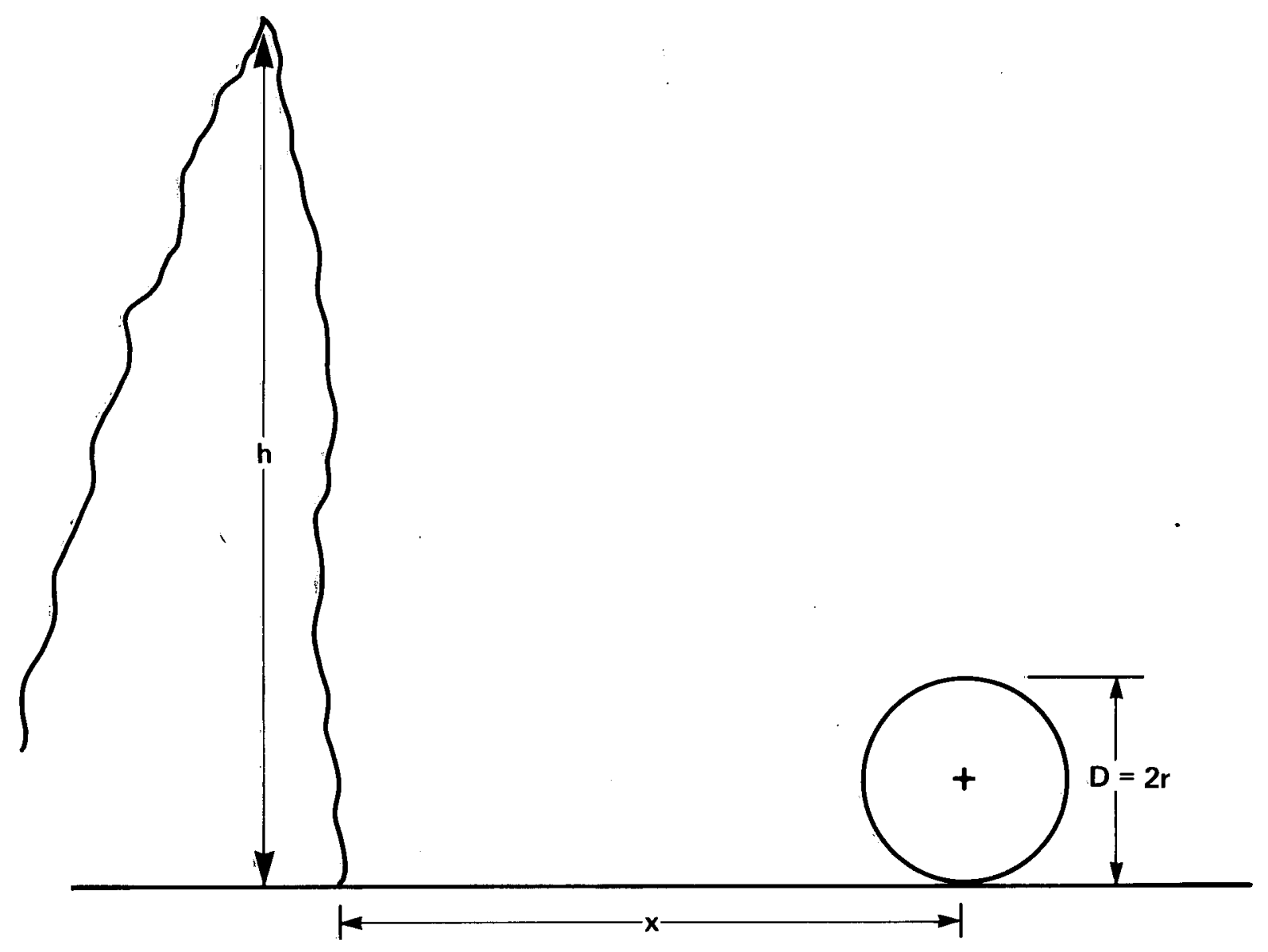

Figure F-13 Cask on ground--distant from flame front. 


$$
\begin{aligned}
A_{s} & =r(\pi+\psi), \mathrm{ft}^{2} / \mathrm{ft} \\
\psi & =\tan ^{-1}\left(\frac{h-4}{x}\right)-\tan ^{-1}\left(\frac{r}{h^{2}+x^{2}-2 h r}\right), \text { radians }
\end{aligned}
$$

and all other terms are as previously defined.

Evaluating these expressions over a range of distances relative to the cask diameter results in the family of curves for the heat load on the cask relative to the engulfing fire value versus the separation distance divided by the cask diameter as shown in Fig. F-14. The total heat load drops rapidly from the reference regulatory value as the distance from the fire increases. In addition at distances removed from the flame, a lower value of emissivity for the cask surface is likely since a blackening of the surface from soot in the flame is less probable, leading therefore to even lower heat loading. In addition to lower heat loading, the cask involved in a nonengulfing fire is able to reject heat by reradiation and natural convection to the environment. 


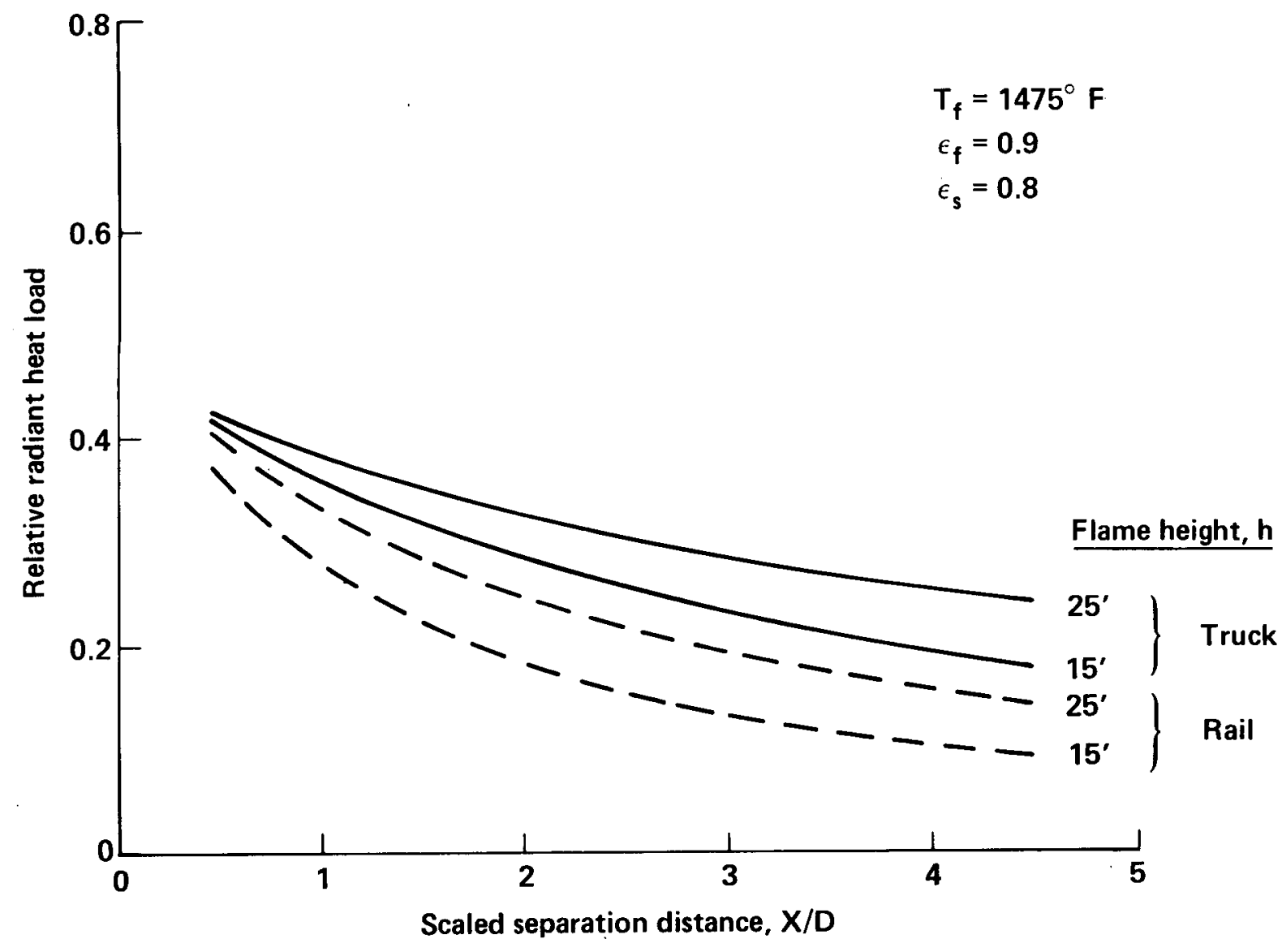

Figure F-14 Fraction of heat load from nonengulfing fires. 


\section{References}

F.1 P. J. Burns, TACO-2D - A Finite Element Heat Transfer Code, Lawrence Livermore National Laboratory Report, Livermore, CA, UCID-17980, Rev. 2, January 1982.

F.2 M. G. Vigil, A. A. Trujillo, H. R. Yoshimura, HNPF Spent Fuel Cask Temperature Response; Torch Impinging on Water Filled Neutron Shield, Sandia National Laboratory, Albuquerque, NM, SAND82-0704, March 1982.

F.3 W. H. McAdams, Heat Transmission, McGraw-Hi1l, New York, NY, 1954.

F.4 J. P. Holman, Heat Transfer, McGraw-Hill, New York, NY, 1976.

F.5 R. K. Clarke, et. al., Severities of Transportation Accidents, Sandia National Laboratory, Albuquerque, NM, SLA-74-001, July 1976.

F.6 Final Report on Assessment of Rail Cask Performance in Railroad Accident Environments, Ridihalgh, Eggers and Associates, Columbus, OH, June 1977.

F.7 L. H. Russe1, and J. A. Canfield, "Experimental Measurement of Heat Transfer to a Cylinder Immersed in a Large Aviation-Fuel Fire", Journal of Heat Transfer, August 1973.

F.8 B. E. Bader, "Heat Transfer in Liquid Hydrocarbon Fuel Fires", Proceedings-International Symposium for Packaging and Transportation of Radioactive Materials, January 1965.

F.9 B. Hagglund, and L. Persson, The Heat Radiation from Petroleum Fires, FOA Report C 20126-d6(A3), Forsvarets Forskningsansta1t, Huvudenhet 2, July 1976.

F.10 L. Orloff, and J. de Ris, Froude Modeling of Pool Fires, Technical Report FMRC J. I. OHON3. BU RC81-BT-9, Factory Mutual Research, October 1983. 
F.11 R. Siege1, and J. R. Howe11, Thermal Radiation Heat Transfer, McGrawHill, New York, NY, 1972. 
APPENDIX G

Probability Estimation Techniques

\section{G.1 Introduction}

Assessment of the probability of the potential responses of a cask to various accident scenarios depends on (1) the description of the distributions of the accident parameters given an accident, and (2) integration of these probability distributions over the appropriate subranges of values of the accident parameters. An important accident parameter is the velocity of the transporting vehicle, either truck or train, at the time of the accident. The distribution of vehicle velocities at the point of an accident is unknown; however, there are data which can be used to estimate the distribution of velocities either subjectively, as in the case of trucks, or recorded, as for trains. In Section G.2, a method of estimation, called maximum entropy, is described for developing the distribution of vehicle velocities using observed velocities at past accidents. This method was applied to both trucks and trains to develop estimates of the appropriate probability distributions of velocity. Given descriptions of the distributions of vehicle velocities and other accident parameters, assessment of the probability of potential cask responses involves integrating several probability functions. The integration process is described in Section G.3. Specifically, Section G.3 describes an approximation, based on sums of discrete probabilities, to the integration of the continuous distributions.

\section{G.2 Maximum Entropy Method of Estimation}

Given the historical data on velocities of vehicles involved in accidents, there are several methóds, such as least squares, maximum likelihood, and density estimation, which can be used to estimate the probability distribution of velocities." Most methods require some identification of the form (family) of the probability distributions. Several distributions and mixtures of distributions were fitted to the accident data but no one family consistently fit all the data. 
Since a specific parametric family of distributions was not readily identifiable, a reasonable approach is to evaluate a nonparametric estimate of the probability distributions of velocity. Although not as powerful, i.e., it has a greater uncertainty, the nonparametric approach allows the data to determine the form of the distribution of velocities rather than forcing the distribution to be of some specific type, e.g., normal or lognormal. If a specific distribution is used and it is not correct, then estimates of probabilities derived from the incorrect distribution can be biased significantly. Thus, we chose to estimate the distributions of velocity nonparametrica11y.

To determine a nonparametric estimate of the distributions of velocity, we based the estimates on the maximum entropy method of estimation. This approach is based on information theory and provides a procedure for estimating a probability distribution, with maximum entropy, consistent with the information available about a random variable. Subject to certain conditions and the appropriate interpretation of probability, .1 it can be shown that the entropy function

$$
H\left(p_{1}, \ldots, p_{K}\right)=-\sum_{k=1}^{K} p_{i} \log p_{i}
$$

measures the amount of "uncertainty" represented by a probability distribution $\left(\hat{p}_{1}, \ldots, \mathrm{p}_{K}\right)$ for a variable $x$ (where it is assumed that $x$ is discrete and has range $\left.x_{1}, \ldots, x_{k}\right)$. Given some information about the distribution of $x$, such as its expected value and variation or uncertainty, a reasonable criterion for estimating the probability distribution $p_{1}, \ldots, p_{K}$ is to maximize the entropy function, (G.1), consistent with the information available, i.e., if $\mu_{0}, \sigma_{0}^{2}$ are the expected value and variance, to estimate $p_{1}, \ldots, p_{k}$ such that

$$
\sum_{k=1}^{k} x_{k} p_{k}=\mu_{0}
$$




$$
\sum_{k=1}^{k}\left(x_{k}-\mu_{0}\right)^{2} p_{k}=\sigma_{0}^{2}
$$

That is, an estimate of the probability distribution $p_{1}, \ldots, p_{K}$ is the set of values $\tilde{p}_{1}, \ldots, \tilde{p}_{k}$ such that

$$
\begin{gathered}
H\left(\tilde{p}_{1}, \ldots, \tilde{p}_{k}\right)=\max H\left(p_{1}, \ldots, p_{K}\right) \\
\left(p_{1}, \ldots\right)
\end{gathered}
$$

subject to the constraints

$$
\begin{gathered}
\sum_{k=1}^{k} p_{k}=1 \\
\sum_{k=1}^{k} x_{k} p_{k}=\mu_{0} \\
\sum_{k=1}^{k}\left(x_{k}-\mu_{0}\right)^{2} p_{k}=\sigma_{0}^{2} .
\end{gathered}
$$

Introducing Lagrangian multipliers $\lambda_{0}, \lambda_{1}, \lambda_{2}$ " associated with the three constraints, the estimated probabilities are

$$
\tilde{p}_{i}=e^{-\left[\lambda_{0}+\lambda_{1} x_{i}+\lambda_{2}\left(x_{i}-\mu_{0}\right)^{2}\right]}
$$


where

$$
\lambda_{0}=\log \sum_{k=1}^{k} e^{-\left[\lambda_{1} x_{k}+\lambda_{2}\left(x_{k}-\mu_{0}\right)^{2}\right]}
$$

and $\lambda_{1}, \lambda_{2}$ are solutions to the equations

$$
\begin{aligned}
n^{-1} \sum_{k=1}^{k} x_{k} e^{-\left[\lambda_{1} x_{k}+\lambda_{2}\left(x_{k}-\mu_{0}\right)^{2}\right]}=\mu_{0} \\
n^{-1} \sum_{k=1}^{k}\left(x_{k}-\mu_{0}\right)^{2} e^{-\left[\lambda_{1} x_{k}+\lambda_{2}\left(x_{k}-\mu_{0}\right)^{2}\right]}=\sigma_{0}^{2}
\end{aligned}
$$

where

$$
n=\sum_{k=1}^{k} e^{-\left[\lambda_{1} x_{k}+\lambda_{2}\left(x_{k}-\mu_{0}\right)^{2}\right]}
$$

Thus, a discrete probability distribution can be constructed which maximizes entropy and which equals the specified mean and variance. In our application of the methodology, we used the mean and the variance of the historical data on velocities as the available information.

If the variable $x$ is considered to be a continuous variable, i.e., its probability distribution has a density function, the estimated density function $f(x)$ can be approximated, based on maximizing entropy, using the identity

$$
d p=f(x) d x
$$


Approximating the density function by a discrete relative histogram $\left[\left(\Delta p_{k}, \Delta x_{k}\right): k=1, \ldots, k\right]$,

$$
f\left(x_{k}\right) \stackrel{\Delta p_{k}}{\Delta x_{k}} .
$$

However, in our notation $\Delta p_{k}=p_{k}$ and, assuming a partition of the (finite) range $R_{x}$ of $X$ into $N$ equal subintervals of length $\Delta x$,

$$
\Delta x=R_{x} / N
$$

the maximum entropy estimate of $f\left(x_{k}\right)$ is

$$
\begin{aligned}
\tilde{f}\left(x_{k}\right) & =\lim _{\Delta \rightarrow 0} \frac{e^{-\left[\Delta \lambda_{0}+\Delta \lambda_{1} x_{k}+\Delta \lambda_{2}\left(x_{k}-\mu_{0}\right)^{2}\right]}}{R_{x} / N} \\
& \cong \frac{e^{-\left[\lambda_{0}+\lambda_{1} x_{k}+\lambda_{2}\left(x_{k}-\mu_{0}\right)^{2}\right]}}{R_{x} / N}
\end{aligned}
$$

for sufficiently small $\Delta$.

The estimated probability distribution, as described by the estimated cumulative distribution function, is based on cumulative sums of the $\tilde{f}\left(x_{k}\right)$ 's, interpolating for $x=x_{k}$. This is the method used to estimate the probability distributions for vehicle velocities prior to and at the point of an accident. The uncertainty of using the sample information for specifying $\mu_{0}$ and $\sigma_{0}^{2}$ was not quantified, nor was the sensitivity investigated for the predicted probabilities of the various response states. Some parametric 
estimates of the distributions of velocities were analyzed, and these would provide some basis for an investigation of sensitivity.

\section{G.3 Discretized Probability Integration}

Estimation of the probability that the response of a cask to an accident is a specific response state, e.g., $R(2,3)$, between $0.2 \%\left(S_{1}\right)$ and $2 \%\left(S_{2}\right)$ strain and between $600^{\circ} \mathrm{F}\left(T_{2}\right)$ and $650^{\circ} \mathrm{F}\left(T_{3}\right)$ lead mid-thickness temperature, is based on evaluating a pair of double integrals of probability distribution and density functions (see Equation 5.23). Some of the probability distribution and density functions are known analytically, but some, for example the distributions of velocities estimated by the method of maximum entropy, are only known numerically. In either case, the integration is complex and cannot be done analytically. Instead, evaluation of the estimated probabilities is based on the identity, given the appropriate conditions,

$$
\int_{a}^{b} H(t) d t=\lim _{\Delta \rightarrow 0} \sum_{k=1}^{K(\Delta)}\left[H\left(t_{k}+\Delta_{u}\right)-H\left(t_{k}-\Delta_{\ell}\right)\right] \Delta t_{k}
$$

$$
=\sum_{k=1}^{K(\Delta)}\left[H\left(t_{k}+\Delta_{u}\right)-H\left(t_{k}-\Delta_{\ell}\right)\right] \Delta t_{k}
$$

for sufficiently small $\Delta$. In this application, the function $H(t)$ itself involves the integral of probability distributions and density functions.

The computer code TASP was developed to perform the necessary summations to approximate the probability integrals (in addition, the code contains all the appropriate probabilities). In each case the code partitions the range of integration into an appropriate number of subintervals to integrate over a probability distribution. When appropriate, the code conservatively evaluates a function at the upper (lower) limit of a subinterval to assure that the estimated probability is conservative. However, the estimate is not overly 
conservative because a reasonable number of subintervals are used for the approximation. Thus, in the context of the inputs, the estimated probabilities are considered good estimates.

\section{G.4 References}

G.1 E. T. Jaynes, "Information Theory and Statistical Mechanisms", Physical Review, Vol. 106, No. 4, May, 1957, pp. 620-630. 


\section{APPENDIX H}

List of Figures

Page

H-1 Impact of weighted steel cylinder into a rigid rail $\ldots \ldots \ldots \ldots \ldots \ldots . . . .5$

H-2 Finite element mesh for one-quarter of the cylinders $\ldots \ldots \ldots \ldots \ldots \ldots . . .6$

H-3 Deformed shapes of the cylinder impacting a rail (DYNA 3-D

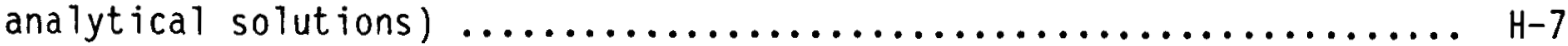

H-4 Mesh of steel nose cone $\ldots \ldots \ldots \ldots \ldots \ldots \ldots \ldots \ldots \ldots \ldots \ldots \ldots \ldots \ldots \ldots$

$\mathrm{H}-5$ Location of tied and sliding interfaces $\ldots \ldots \ldots \ldots \ldots \ldots \ldots \ldots \ldots \ldots$

H-6 Sequence of deformed configurations $\ldots \ldots \ldots \ldots \ldots \ldots \ldots \ldots \ldots \ldots \ldots$

$\mathrm{H}-7$ Computed and experimental force-displacement curve. The steps in the curves correspond to void closures $\ldots . \ldots \ldots \ldots \ldots \ldots \ldots \ldots . . .11$

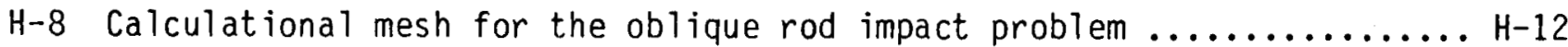

H-9 Deformed shapes of a rod impacting an oblique rigid wall $\ldots \ldots \ldots \ldots \ldots$ H-13

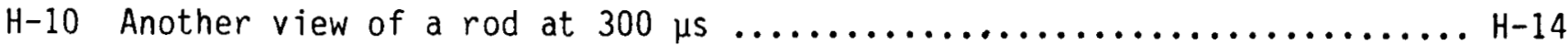

$\mathrm{H}-1 \mathrm{l}$ Final profiles at $3000 \mu \mathrm{s}$ (a) experiment and (b) computed .......... H-15 


\section{APPENDIX $\mathrm{H}$}

Benchmarking for Computer Codes Used in Impact Analyses

\section{H.1 Introduction}

Several computer codes were used in the structural impact analyses to estimate cask responses for the various accidental impact loading conditions in this study. Impact is a governing loading condition in the cask structural evaluation. The results and conclusions stated in this report rely on the adequacy of these codes to estimate structural response. Impact is a loading condition which can generate large amounts of energy during a very short duration of impact. During the impact, energy changes form from potential into kinetic, ard into strain energy. After the initial impact, the cask has a potential for bouncing back into the air depending on the target hardness and the property of impact limiters. Rigid body motion is involved during this process. In order to estimate the structural damage due to the second impact, i.e., the other end of the cask hitting the target after bouncing around in the air, the computer code needs to have a special capability of handling rigid body motion. Most of the finite element computer codes available today cannot handle the rigid body motion and, therefore, were not selected for this study. To assess cask response to the impact orientation, i.e., the angle between the cask longitudinal axis and the target surface, the selected computer codes need to have the capability of handling impact at an angle. Impact limiters play an important role in cask response. During impact, the limiter will enter a nonlinear region. The selected computer codes need to be capable of handling nonlinear impact-limiter responses.

The representative casks selected in this study use a lead layer for shielding. In order to model the lead behavior inside the inner and outer steel shells, the computer codes need to be capable of handiing sliding between two surfaces of different materials. Not every computer code can satisfy all these specified requirements. Certain computer codes may be capable of meeting partial requirements. It is necessary that the user understands the limitations of the codes selected. 
Three computer codes were selected to perform various types of impact analysis in this study. They are DYNA 2-D/3-D, NIKE 2-D/3-D (the 2D/3D designation indicating that either two-dimensional or three-dimensiona 1 modeling can be performed), and IMPASC (part of the SCAN system). All three codes were developed and maintained at Lawrence Livermore National Laboratory through other programs in the Laboratory. The limitations of each code are understood. During the course of calculating cask response, the analytical group worked very closely with the code development group. In many cases, the codes were modified to suit the specific needs of this study. There is high confidence that these codes were properly used within code capability in calculating cask response when subjected to impact loads. The qualifications of users is only part of the concerns in assuring adequate analytical solutions.

The next. question is how can the selected computer codes simulate the impact conditions and the structural response. To answer this question, computer codes are generally benchmarked by comparing their results against one or more of the following: (1) results from closed form engineering solutions, (2) test data, and (3) other computer codes which have been benchmarked. This appendix presents benchmark codes for DYNA 3-D. The other codes, DYNA 2-D, NIKE 2D/3-D, and IMPASC have been benchmarked against DYNA 3$D$, hence this benchmark test also generally applies to the other codes.

To date, these codes have not been benchmarked for predicting lead sliump. Although at least one foreign country has performed impact tests with lead casks and used DYNA 2-D for benchmarking, these results are proprietary and cannot be disclosed. Therefore all of the calculations done in this study with DYNA and NIKE were performed assuming conservative lead properties and boundary conditions that over predict lead slump and the strain on the inner wall of the representative cask models.

\section{H.2 Benchmark Cal ibrations for DYNA 3-D}




\section{H.2.1 Impact of Cylinder into Rail}

The steel cylinder shown in $F i g . ~ H-I$ is impacted into a long rigid rail at $1676 \mathrm{~cm} / \mathrm{sec}$. Attached to the ends of this cylinder are weights of $62.3 \mathrm{M}$ dyne. An experimental test was conducted and the final configuration was measured.

One quarter of the cylinder was modeled by using DYNA 3-D with two planes of symmetry using the mesh illustrated in Fig. H-2. This mesh contains 3432 elements. Elastic-perfectly plastic behavior was assumed for the steel with a yield strength of 0.0131 Mbar.

Deformed shapes at approximately millisecond increments are shown in Fig. $H-3$. At $6.4 \mathrm{~ms}$ the cylinder can be seen to have completely rebounded with its final deformed shape. A maximum residual dent of 1.53 inches was calculated. A maximum dent of 1.44 inches was measured at the same location in an experimental test.

\section{H.2.2 Nose Cone Analys is}

Figure H-4 shows the DYNA 3-D mesh (6074 nodes, 4356 elements) used to model a steel (yield strength $=0.0048 \mathrm{Mbar}, E_{t}=0.0138 \mathrm{Mbar}$ ) nose cone that, on impact, has been designed by Sandia Laboratories in Livermore to limit the resultant force transmitted to the aft section. ${ }^{H .1}$ The mass of the aft section is mocked with a high-density material, $131,477 \mathrm{gm} / \mathrm{cm}^{3}$, in the top rows of elements.

This problem is interesting from a code development viewpoint because it exercises the sliding interface logic. Five interfaces are defined of which two are tied. The locations of these interfaces are depicted in Fig. H-5.

Deformed shapes at 3,000 $\mu$ s intervals are shown in Fig. H-6. At $15,000 \mu \mathrm{s}$ the peak deformation is reached and the nose cone begins to rebound.

Comparisons with experimental data from a static test showed excellent agreement with the calculation. ${ }^{H .1}$ The final shape obtaining in the experiment was very close to the final computed shape. In Fig. H-7, the computed force deflection curve from DYNAP is compared to the experiment. only minor discrepancies exist. 


\section{H.2.3 Oblique Impact of Rod}

An aluminum rod $30.5 \mathrm{~cm}$ long and $0.638 \mathrm{~cm}$ in diameter impacts a rigid wall oriented at $10^{\circ}$ at a velocity of $20,170 \mathrm{~cm} / \mathrm{sec}$. The material behavior is simulated with material model 11 using the properties defined in UCRL80465. H.2 Fig. H-8 shows the DYNA 3-D calculational mesh.

The computed results showed good agreement with the experimental profiles up to $600 \mu \mathrm{s}$. At later times the experiments showed more curvature in the rod. Four factors probably contributed to these late time discrepancies.
- coarse zoning,
- inaccurate material properties,
- rigid wall approximation to armor plate,
o lack of interface friction.

Figure $\mathrm{H}-9$ shows a sequence of deformed configurations. Figure. H-10 shows a view of $300 \mu$ s to illustrate the cross-sectional zoning. Figure H-11 shows the residual experimental profile for comparison to the computed result at $3,000 \mu \mathrm{s}$. 


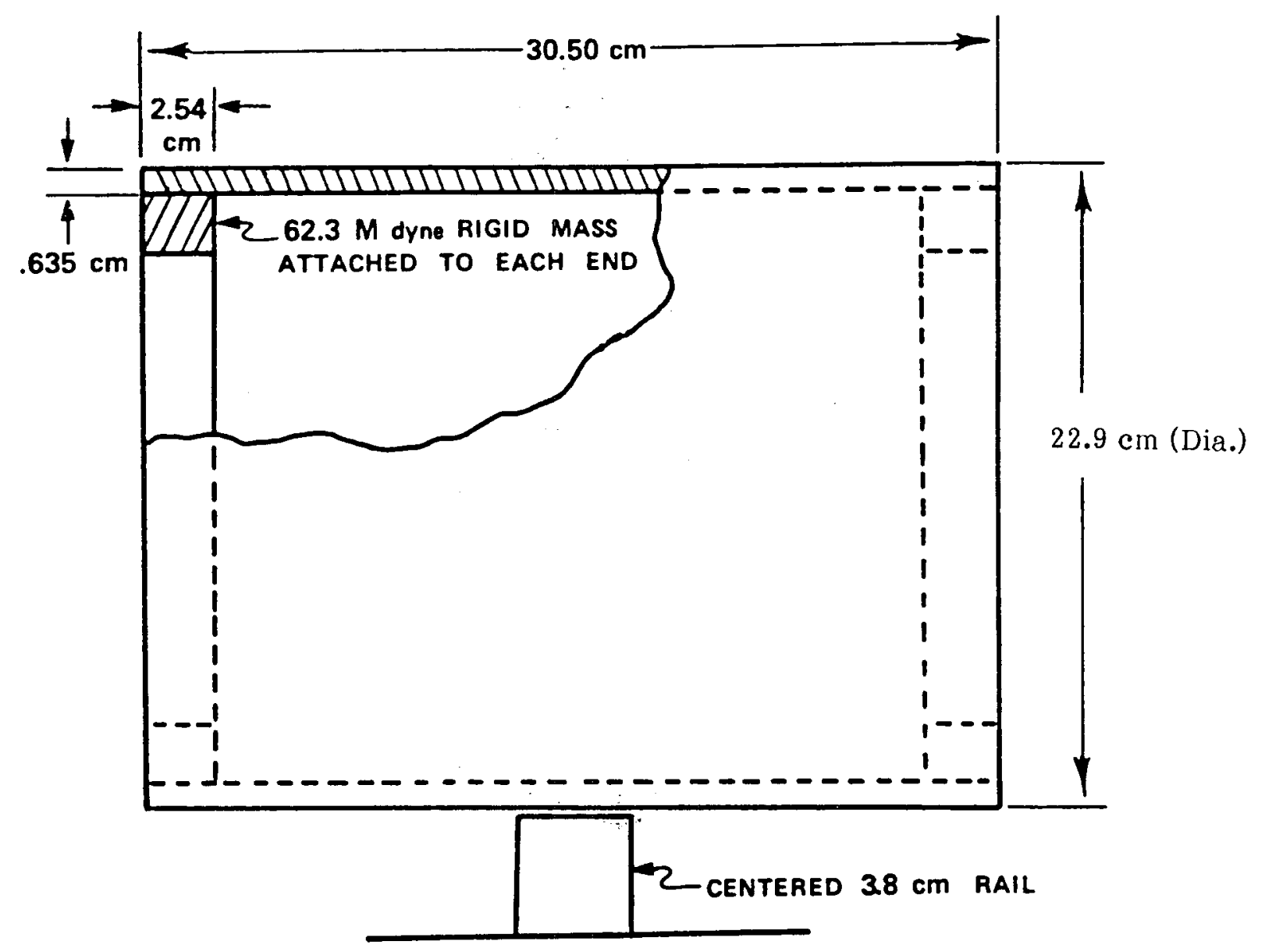

Figure H-1 Impact of weighted steel cylinder into: a rigid rail. 


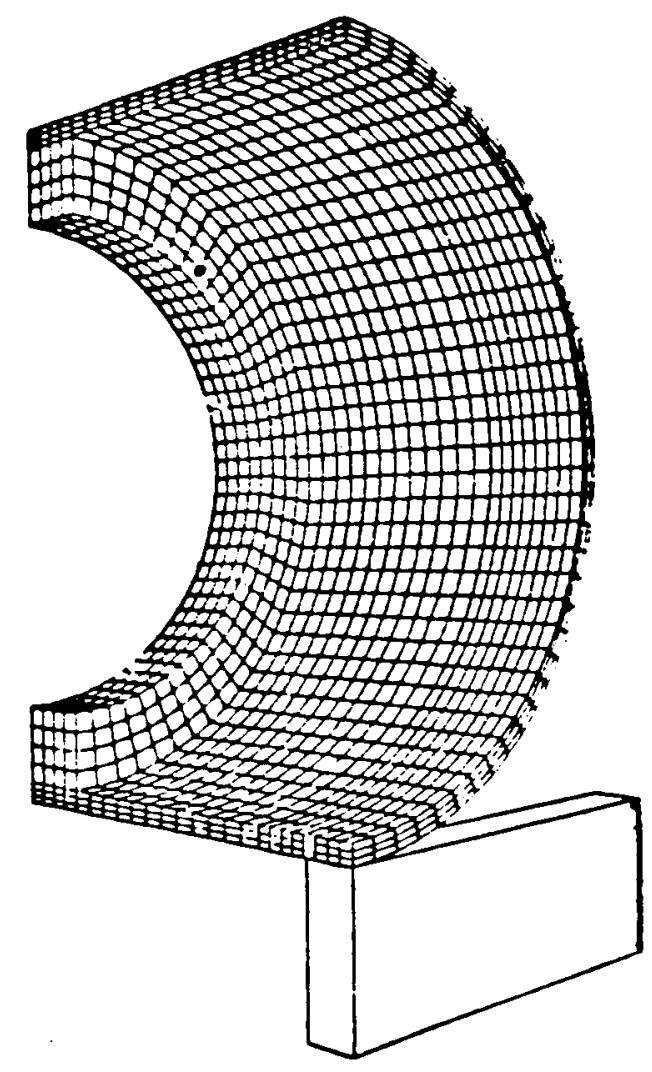

Figure H-2 Finite element mesh for one-quarter of the cylinders. 

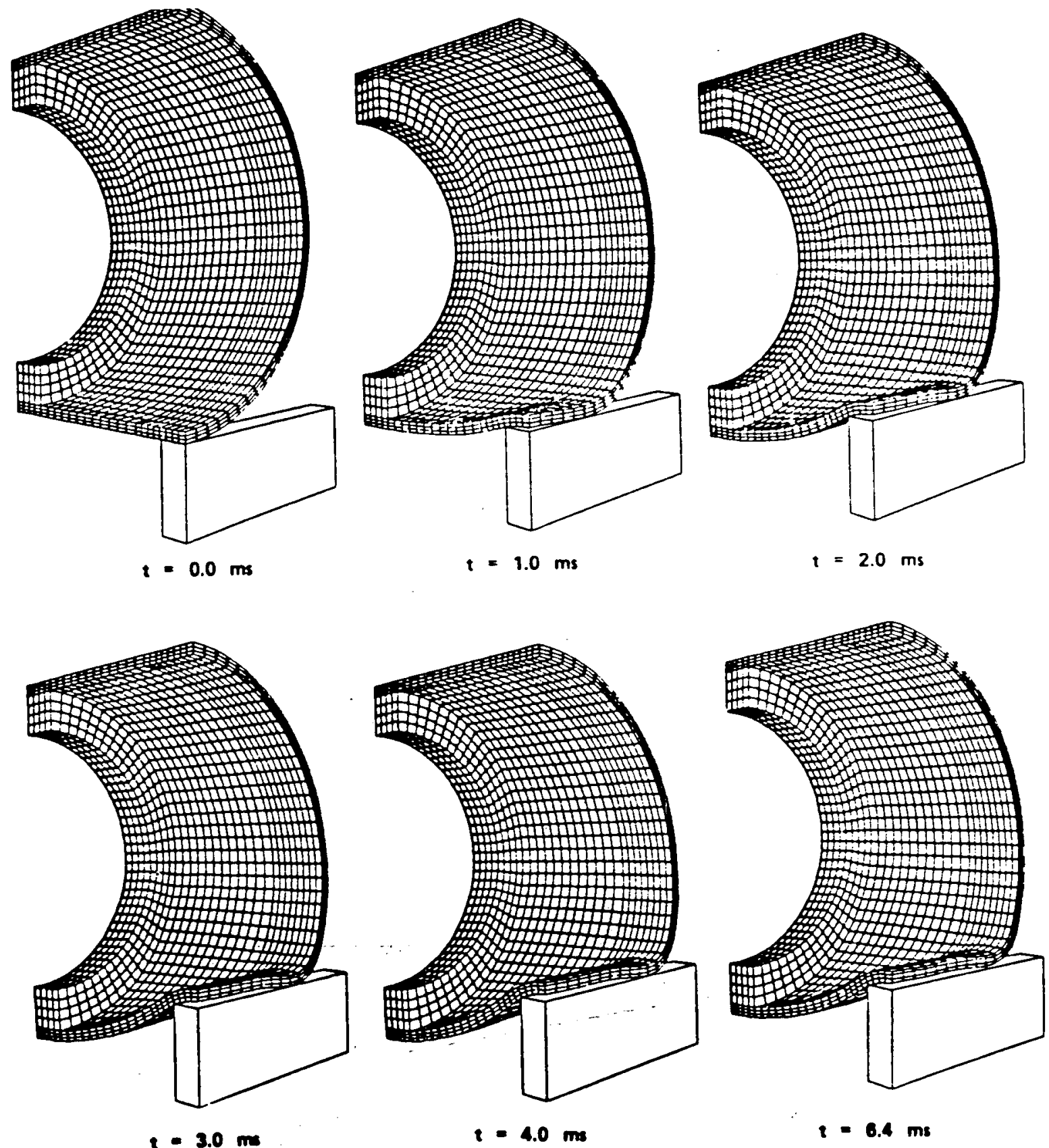

Figure H-3 Deformed shapes of the cylinder impacting a rail (DYNA 3-D analytical solutions). 


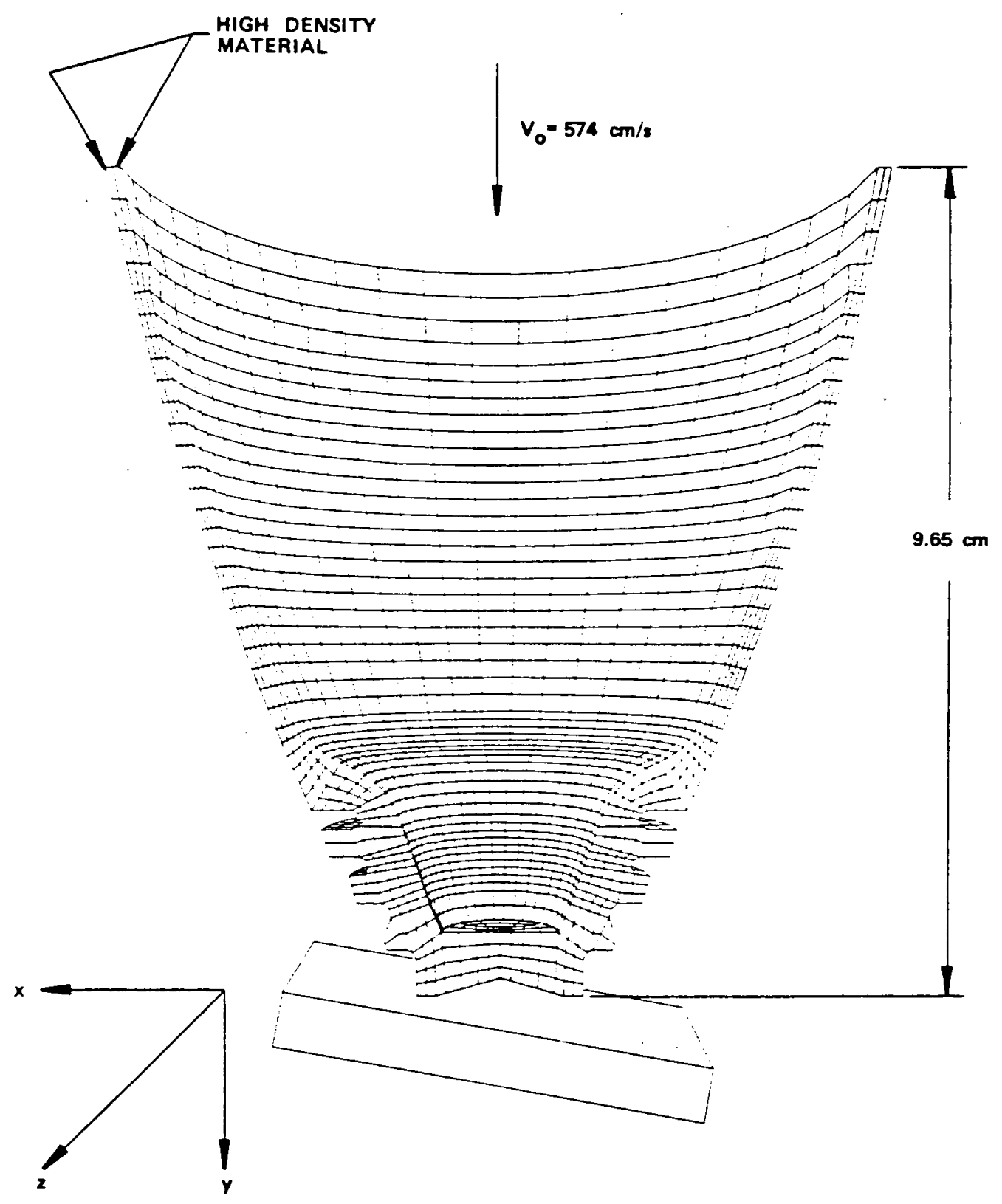

Figure $\mathrm{H}-4$ Mesh of steel nose cone. 


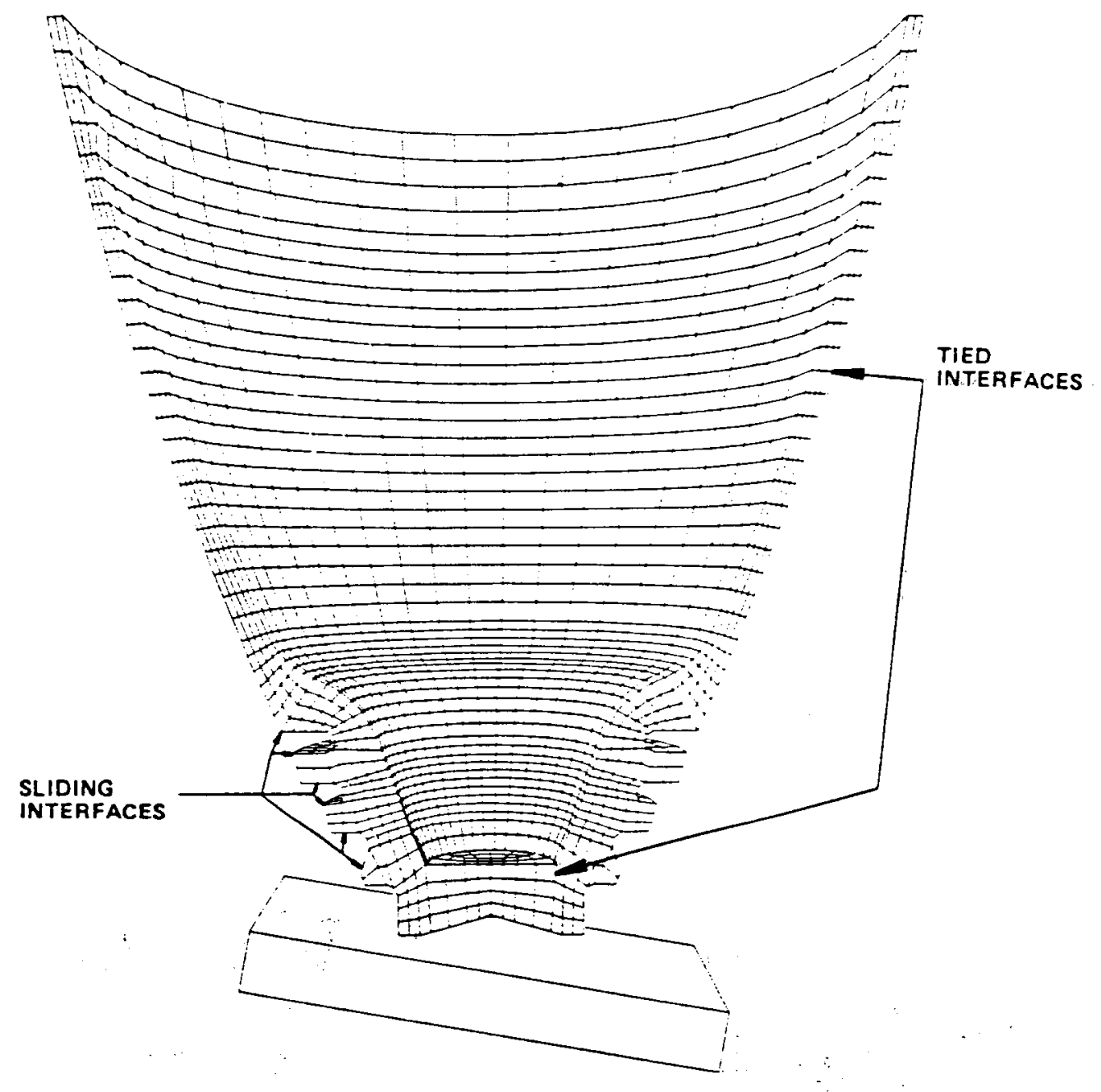

Figure H-5 Location of tied and sliding interfaces. 


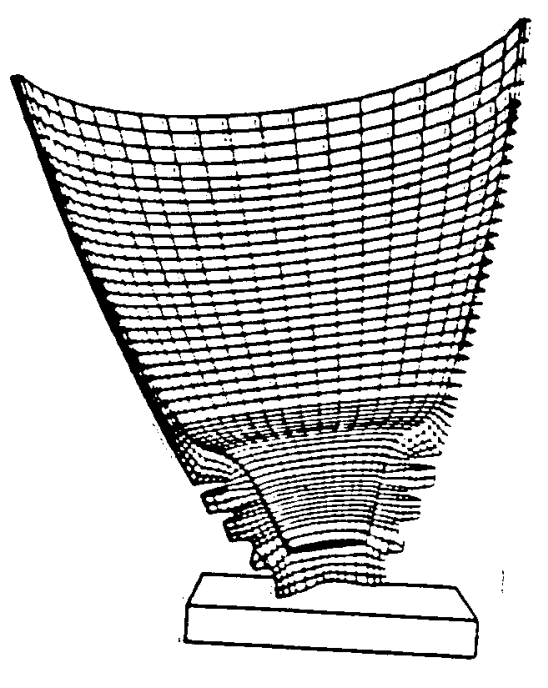

$t=0.0$

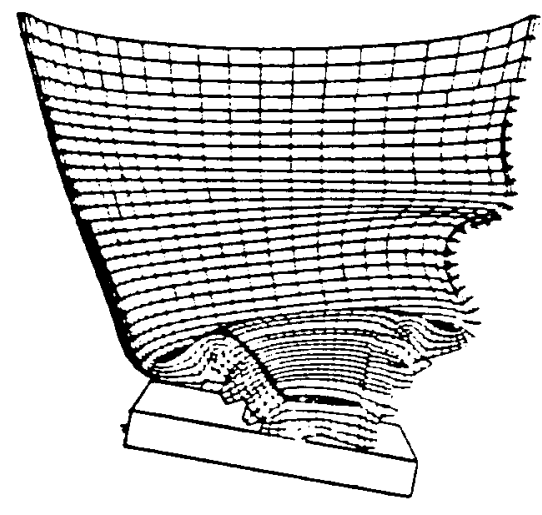

$t=6000 \mu \mathrm{s}$

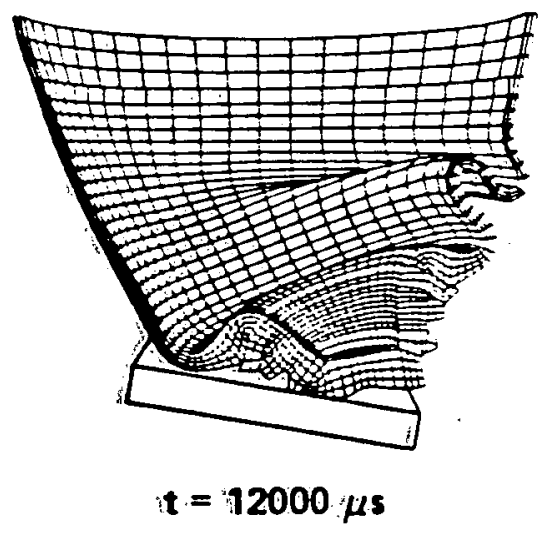

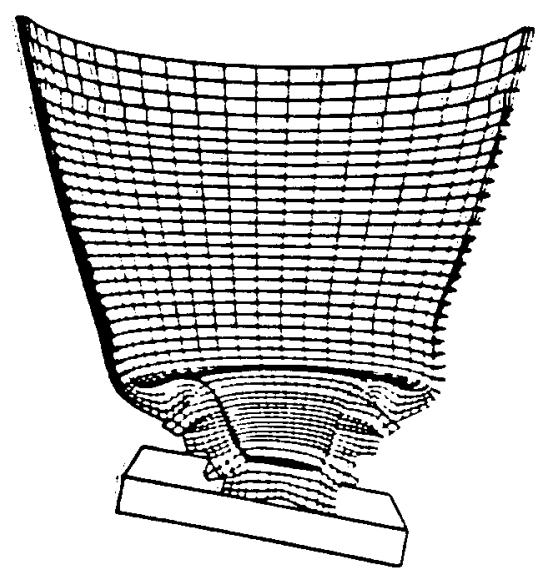

$t=3000 \mu s$

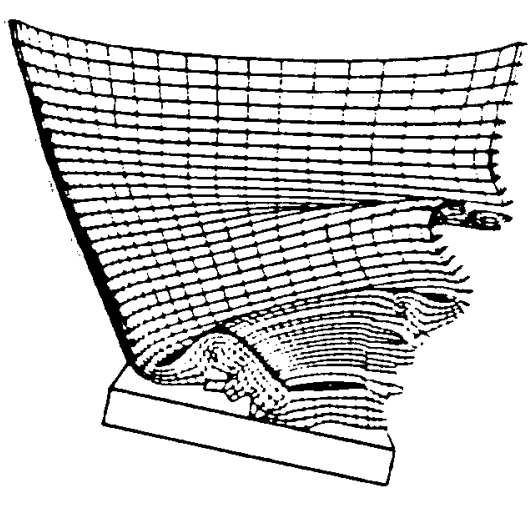

$t=9000 \mu \mathrm{s}$

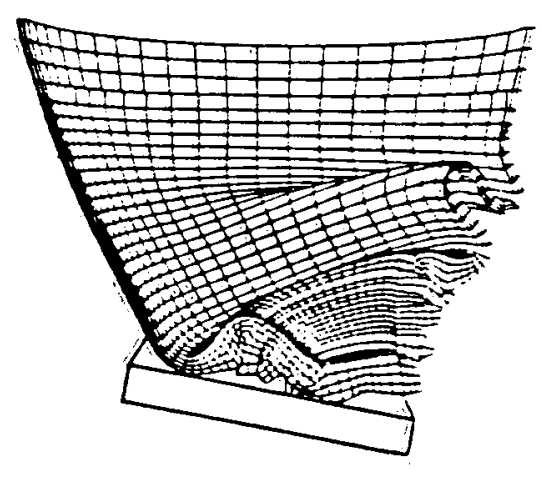

$t=15000 \mu \mathrm{s}$

Figure $H^{-6} 6$ Sequence of deformed configurations. 


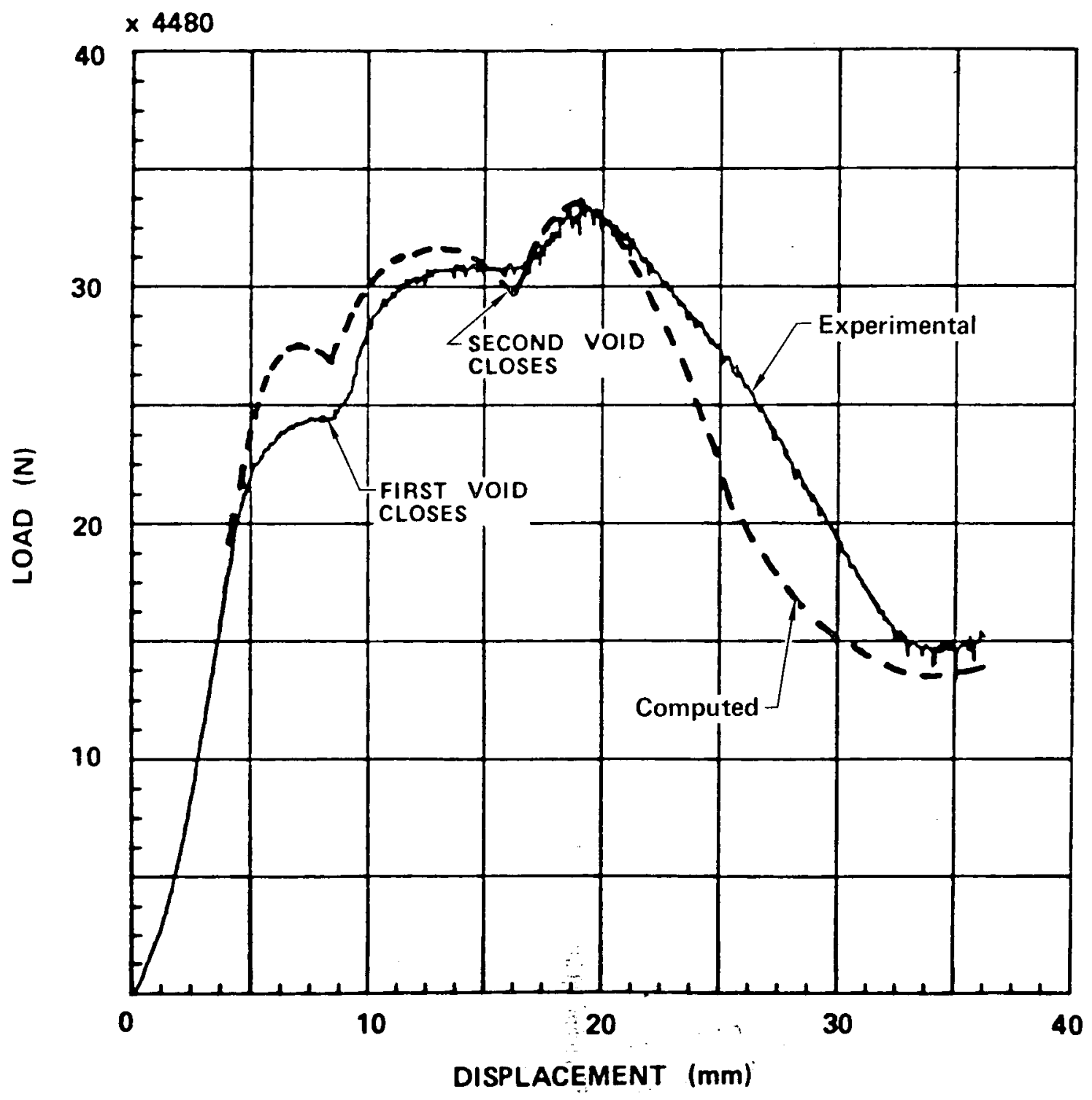

Figure H-7 Computed and experimental force-displacement curve. The steps in the curves correspond to void closures. 


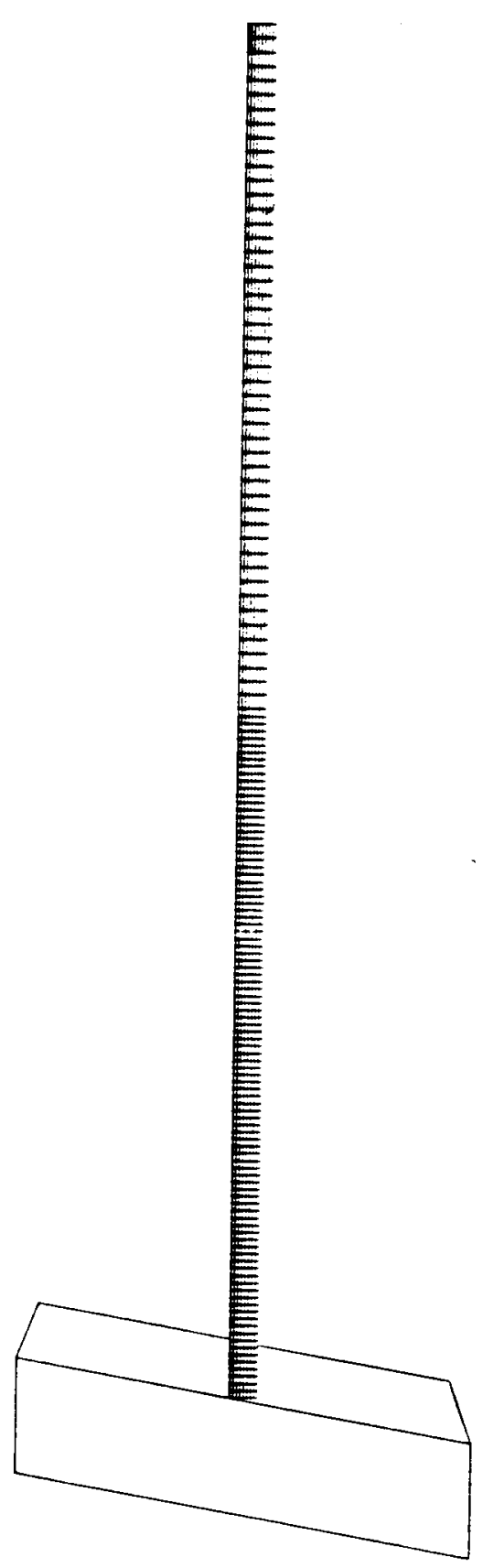

Figure H-8 Calculational mesh for the oblique rod impact problem. 


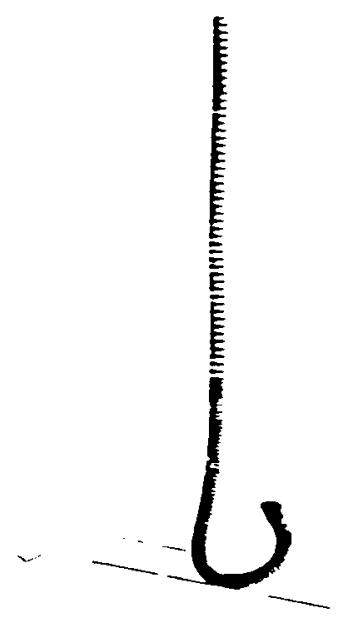

$t=0.0 \mu \mathrm{s}$

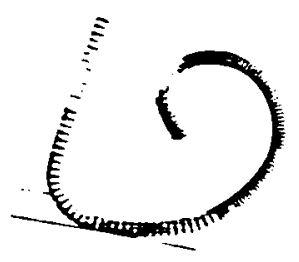

$t=1600 \mu \mathrm{s}$ $t=400 \mu \mathrm{s}$

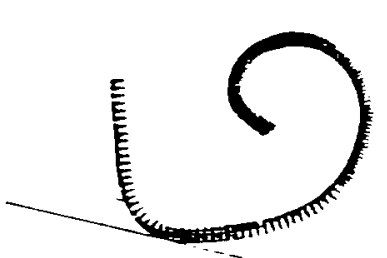

$t=2000 \mu \mathrm{s}$

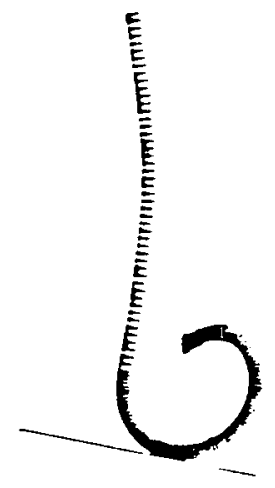

$t=800 \mu \mathrm{s}$

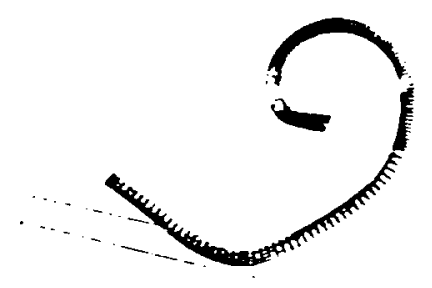

$t=2400 \mu \mathrm{s}$

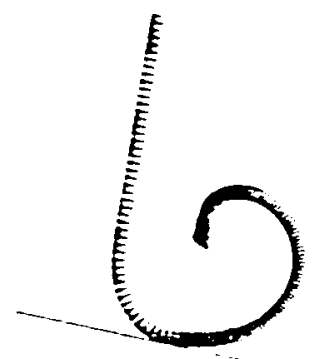

$t=1200 \mu \mathrm{s}$

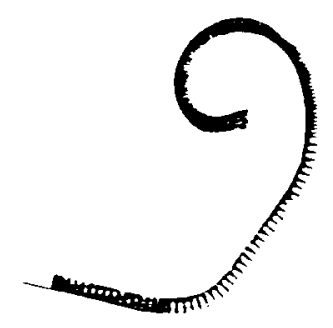

$t=2800 \mu \mathrm{s}$

Figure H-9 Deformed shapes of a rod impacting an oblique rigid wall.

$\mathrm{H}-13$ 


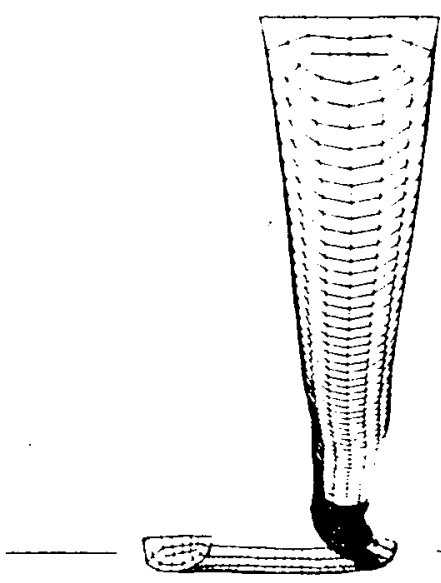

Figure $\mathrm{H}-10$ Another view of a rod at $300 \mu \mathrm{s}$.

$$
H-14
$$



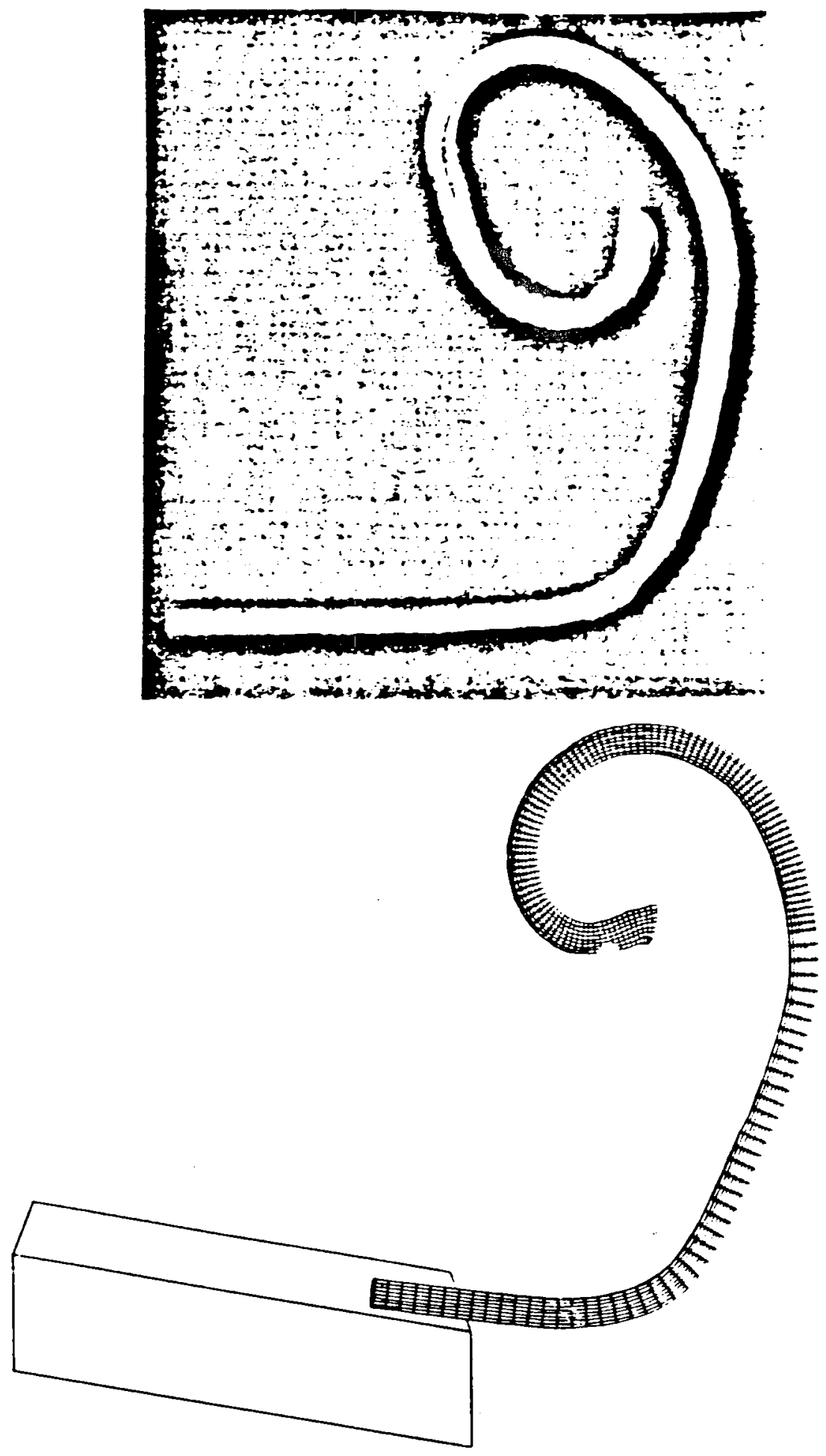

Figure H-11 Final profiles at $3000 \mu \mathrm{s}$ (a) experiment and (b) computed. 


\section{References}

H.1 M. Chiesa and M. Callabresi, "Nonlinear Analysis of a Mitigating Steel Nose Cone," Computers and Structures, Vol. 13, Sandia National Laboratories, Livermore, CA, 1981, p. 295.

H.2 D. J. Steinberg and M. W. Guinan, A High-Strain Constitutive Model for Metals, Lawrence Livermore National Laboratory, Livermore, CA, UCRL80465, 1978. 


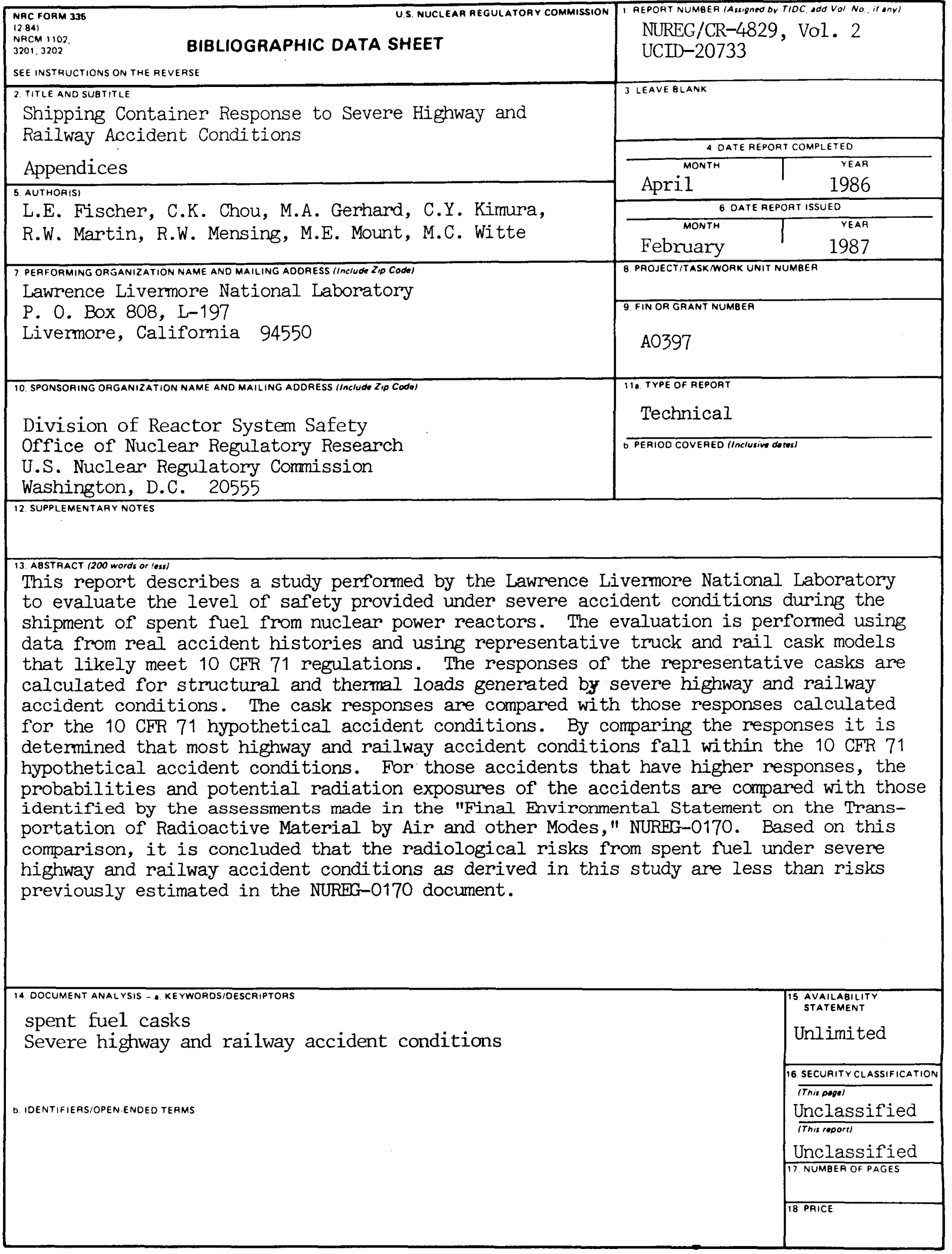

